\title{
Development of Zwitterionic Chromophores and Nonlinear Optical Polymers for Electro-Optic and Near Infrared Chemosensor Applications
}

\author{
By \\ Wen Hui Hao

\begin{abstract}
A thesis submitted to
the Faculty of Graduate and Postdoctoral Affairs

In partial fulfillment of the requirements for the degree of

Doctor of Philosophy in Chemistry
\end{abstract}
Carleton University
Ottawa, Ontario

July 2011

(C) Copyright 2011, Wen Hui Hao 
Library and Archives

Canada

Published Heritage

Branch

395 Wellington Street Ottawa ON K1A ON4 Canada
Bibliothèque et

Archives Canada

Direction du

Patrimoine de l'édition

395 , rue Wellington

Ottawa ON K1A ON4

Canada
Your file Votre référence

ISBN: 978-0-494-83214-1

Our file Notre reférence

ISBN: $978-0-494-83214-1$
NOTICE:

The author has granted a nonexclusive license allowing Library and Archives Canada to reproduce, publish, archive, preserve, conserve, communicate to the public by telecommunication or on the Internet, loan, distribute and sell theses worldwide, for commercial or noncommercial purposes, in microform, paper, electronic and/or any other formats.

The author retains copyright ownership and moral rights in this thesis. Neither the thesis nor substantial extracts from it may be printed or otherwise reproduced without the author's permission.
AVIS:

L'auteur a accordé une licence non exclusive permettant à la Bibliothèque et Archives Canada de reproduire, publier, archiver, sauvegarder, conserver, transmettre au public par télécommunication ou par l'Internet, prêter, distribuer et vendre des thèses partout dans le monde, à des fins commerciales ou autres, sur support microforme, papier, électronique et/ou autres formats.

L'auteur conserve la propriété du droit d'auteur et des droits moraux qui protège cette thèse. $\mathrm{Ni}$ la thèse ni des extraits substantiels de celle-ci ne doivent être imprimés ou autrement reproduits sans son autorisation.
In compliance with the Canadian Privacy Act some supporting forms may have been removed from this thesis.

While these forms may be included in the document page count, their removal does not represent any loss of content from the thesis.
Conformément à la loi canadienne sur la protection de la vie privée, quelques formulaires secondaires ont été enlevés de cette thèse.

Bien que ces formulaires aient inclus dans la pagination, il n'y aura aucun contenu manquant. 


\begin{abstract}
To systematically understand the structure and property relationships and development of zwitterionic chromphores and non linear optical (NLO) polymers for applications in organic electro-optic (EO) materials and near infrared (NIR) fluorescent sensing, two series of zwitterionic chromphores, FDCNs (dicyanomethane-based) and PpQDMs (tricyanoquino-dimethane-based) and their corresponding NLO polymers were designed and synthesized. Incorporation of bulky groups onto the central part of FDCN chromophore was demonstrated as an effective strategy to minimize dipole-dipole molecular interaction. Efficient conjugated bridges were extended from dipyridylium donors and coupled with very strong 3CNQ electron acceptors to afford PpQDM chromophores with very large dipole moments and high hyperpolarizabilities. X-ray crystallographic data, negative solvatochromism, IR and NMR analysis confirm the charge-separated ground states of these two series of zwitterionic chromophores.

In addition, FDCN-MMA NLO polymers (NA2) were synthesized, in which the chromophore contents reached 14 wt \% and exhibited high solubility, thermal stability and film-forming ability. PpQDM-based crosslinkable NLO polyimides were prepared through grafting the hydroxyl-containing PpQDM and BCBO crosslinking unit onto an acid-containing polyimide host. High chromophore loading (13.8 wt \%) onto the polyimide was obtained. The $T_{g}$ values of NLO polyimides were observed to vary due to different pendant groups and different PpQDM loading levels.
\end{abstract}

Thirdly, the poling conditions were optimized through investigations into various factors such as the molecular shape of the chromophores, the polarity of host polymers, poling voltage, and temperature. A large EO coefficient of $70 \mathrm{pm} / \mathrm{V}$ at the 
telecommunication wavelength of $1550 \mathrm{~nm}$ has been obtained with a 5 wt \% PpQDMBen doped PVP polymer using an in situ poling technique. A buffer layer (PEDOT: PSS/PVA) was first introduced into the double layer EO device, and improvement of the poling efficiency of NLO polymer systems was demonstrated.

Furthermore, PpQDMs are NIR fluorescent with large Stokes shifts and show high microenvironmental (pH, solvent polarity) sensitivity. PpQDM-Met (M1) is capable of selectively detecting metal ions $\left(\mathrm{Fe}^{2+}, \mathrm{Cu}^{2+}\right)$ signaled by changes in the absorption and fluorescence. M1/copper complex was prepared and demonstrated to be the first highcontrast colorimetric and NIR fluorescence turn-on molecular probe for sensitive and selective detection of cysteine. 


\section{Acknowledgements}

This thesis would not have been possible without the help and involvement of many people. First of all, I would like to express my deepest gratitude towards my supervisor, Dr. Zhi Yuan (Wayne) Wang, for providing me with such a great opportunity to work on an inspiring project, for his full support, trust and encouragement during my progress. My true appreciation and respect go to him for his knowledge and invaluable guidance in the accomplishment of my study and presentation of this thesis.

Second, my sincere appreciation to Dr. Jane Gao, for all her continuous direction, enthusiasm, expertise, and invaluable advice on various challenges over the last few years. Her devotion and encouragement motivated me during the completion of my Ph.D. study. Special thanks go to my defense committee members Dr. Jianfu Ding, Dr. Jeffrey Manthorpe, Dr. Christopher Boddy and Dr. Steven McGarry.

I wish to thank all my labmates in this project for their pioneer work and fruitful discussions, in particular: Dr. Naiheng Song, Mr. Andrew Beaudin, Dr. Yaowen Bai, Ms. Cara A. M. Weir, Dr. Ying Xiong, Dr. Kunli Wang, Ms. Sabrina Volland and Mr. Kirk M. Mulligan.

I also would like to particularly thank my colleagues, Dr. Gang Qian, Mr. Peter Mirtchev, Ms. Arran McBride and Ms. Skye McBride for your help, contributions and cherished friendships making this thesis work an enjoyable experience.

Many thanks are extended to everyone in Dr. Wang's group: Dr. Hongding Tang, Dr. Erin Todd, Dr. Xun Sun, Dr. Tayebeh Hadizad, Dr. Xianguo Wu, Dr. Shidi Xun, Dr. Geatan LeClair, Mr. Yuxing Cui, Ms. Fatima Hasanain, Mr. Huy Nguyen, Dr. Jia Zheng, Dr. Wenqiang Qiao, Mr. Mathieu Leyre, Dr. Xianzhen Li, Ms. Cathy Wang, Mr. David 
Li, Dr. Ning Wang, Mr. Hana Abu and Mr. Sukanta Saha for providing excellent research environments.

I am thankful to Dr. Hooman Shadnia, for his help with the calculation of dipole moments and molecular hyperpolarizabilities, and Professor Jeff Smith, for his help with HRMS measurement. Thanks to Keith Bourque and Tony O'Neil, for all the help with NMR and IR measurements and to Jim Logan, for his help with computer and instrumental set-up for NLO measurement.

I am grateful to NSERC Canada Graduate Scholarship, Ontario Graduate Scholarship, Ontario Graduate Scholarship in Science and Technology and Carleton University for financial support.

Finally, I want to take this opportunity to appreciate my whole-hearted supportive family: my parents, my brother, my husband Xin Jun Cui and our son, David for their immeasurable love, tremendous support and understanding throughout my studies during the past few years. 


\section{Table of Contents}

Abstract....................................................................................................................... ii

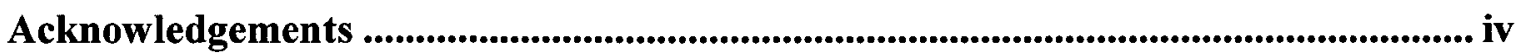

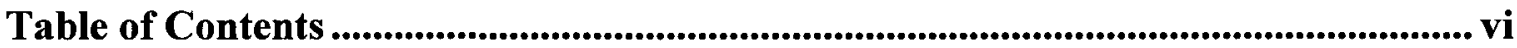

List of Tables ................................................................................................ $\mathrm{x}$

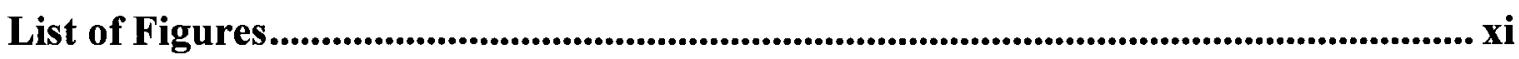

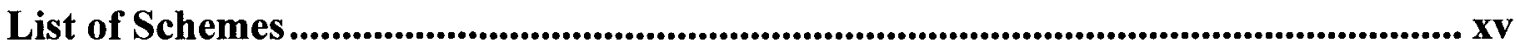

List of Symbols and Abbreviations .............................................................. xvi

Chapter 1 Introduction and Background ................................................................. 1

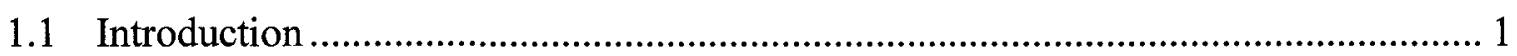

1.2 Electro-Optic Effect and Organic EO Materials ............................................... 3

1.2.1 Basics to Electro-Optic Effect ........................................................ 3

1.2.2 Molecular First Hyperpolarizability ..................................................... 8

1.2.3 Neutral Nonlinear Optical Chromophores........................................... 11

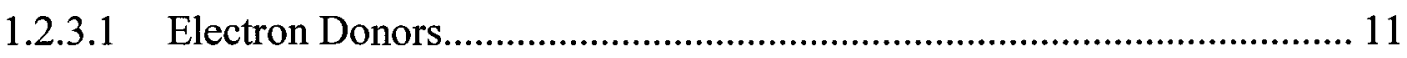

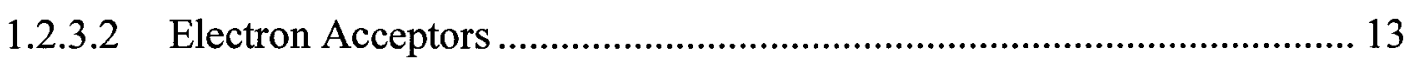

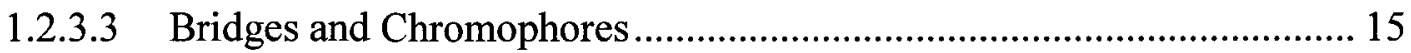

1.2.4 Zwitterionic Nonlinear Optical Chromophores............................................ 17

1.2.4.1 Betain Dyes and Merocyanine Dye................................................... 18

1.2.4.2 Polyphenylenes Bridge /Tricyanofuran-Based Acceptor ...................... 20

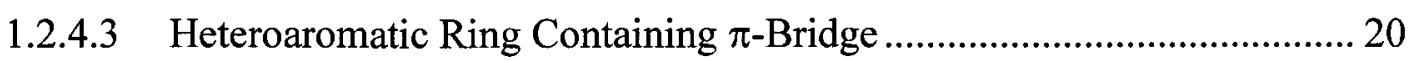

1.2.4.4 $\mathrm{D}^{+}-\pi-\mathrm{A}^{-}$Tertiary Amine/ 3CNQ-Based Zwitterionic Chromophores ... 22

1.2.5 Development of Nonlinear Optical Polymers ......................................... 25

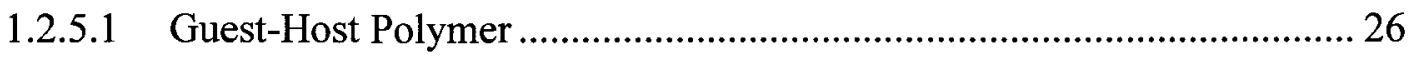

1.2.5.2 Side-Chain and Crosslinkable NLO Polymer ..................................... 27

1.3 Chemosensor Chemistry and Near Infrared Fluorescent Sensors .......................... 29

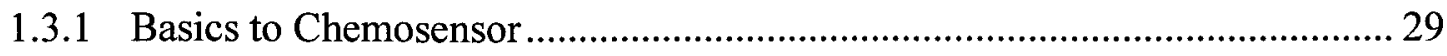

1.3.2 Fluorescent Molecular Sensors of Cations ............................................... 29 
1.3.2.1 Fluorescent Molecular Sensors with Open-Chain Receptor .................... 31

1.3.2.2 Fluorescent Molecular Sensors with Cyclic Receptor ............................. 32

1.3.3 Organometallic Complex-Based Optical Chemosensors .................................. 33

1.3.4 NIR Fluorescent Molecular Sensors......................................................... 35

1.3.4.1 NIR Fluorescent Cyanine Dyes............................................................... 36

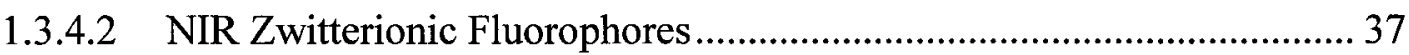

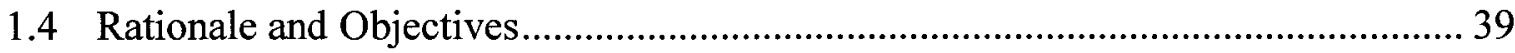

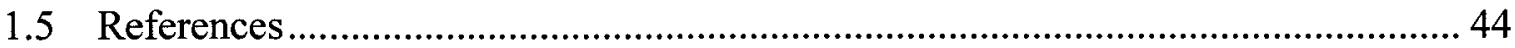

Chapter 2 Synthesis and Characterization of Zwitterionic Chromophores ............ 51

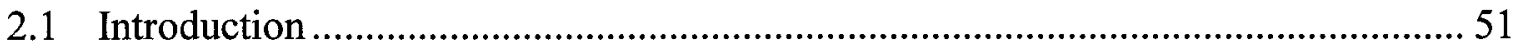

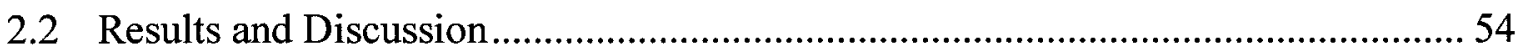

2.2.1 Synthesis and Characterizations of FDCN Chromophores ............................ 54

2.2.1.1 Synthesis of FDCN Chromophores ........................................................ 54

2.2.1.2 Characterizations of FDCN Chromophores ............................................ 55

2.2.1.3 Structure Characterization by X-ray Crystallograph................................ 58

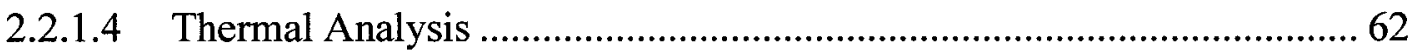

2.2.2 Synthesis and Characterizations of PpQDM Chromophores ......................... 62

2.2.2.1 Synthesis of PpQDM Chromophores.......................................................... 63

2.2.2.2 Characterizations of PpQDM Chromophores .......................................... 65

2.2.2.3 Thermal Stability of PpQDM Chromophores ......................................... 67

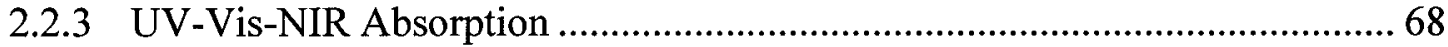

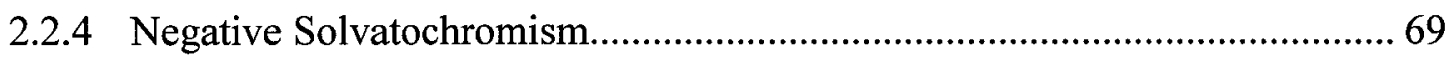

2.2.5 Fluorescence Properties of FDCNs and PpQDM Chromophores .................. 73

2.2.6 Dipole Moment and Molecular Hyperpolarizability ……................................ 78

2.2.7 Acid Sensitivity ......................................................................................... 81

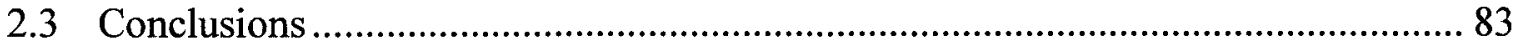

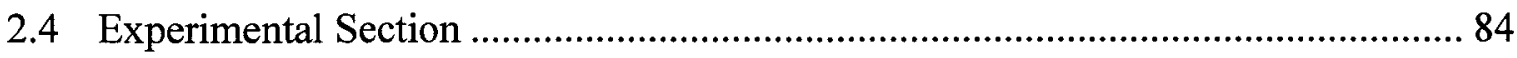

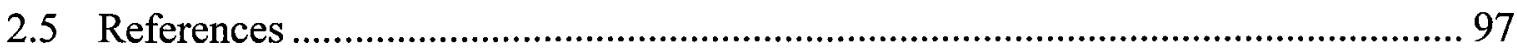

Chapter 3 Synthesis of Zwitterionic Nonlinear Optical Polymers .......................... 100

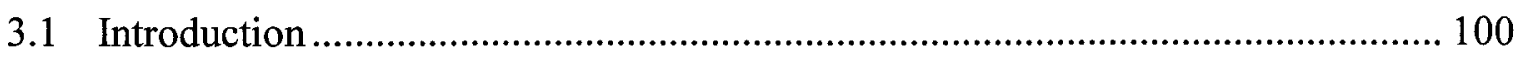


3.2 Synthesis and Characterization of FDCN-based NLO Polymers............................ 101

3.2.1 Synthesis of Cyanoacetate Containing MMA Host Polymers (A1) .............. 101

3.2.2 Synthesis and Characterization of FDCN-A1 NLO Polymers (NA1) .......... 104

3.2.3 Synthesis and Characterization of FDCN-MMA NLO Polymers (NA2).... 106

3.3 Synthesis of PpQDM-Based Crosslinkable NLO Polymers ................................. 109

3.3.1 Host Polymer Selection for Guest-Host System........................................... 109

3.3.2 Design and Synthesis of Polyimides as a Host for PpQDM Chromophores 111

3.3.3 Design and Synthesis of PpQDM-Based Crosslinkable NLO Polymers .... 114

3.3.3.1 Synthesis of PpQDM-Based Crosslinkable NLO Polymers .................. 115

3.3.3.2 Optical Absorption ...................................................................... 120

3.3.3.3 Thermal Analysis ........................................................................... 121

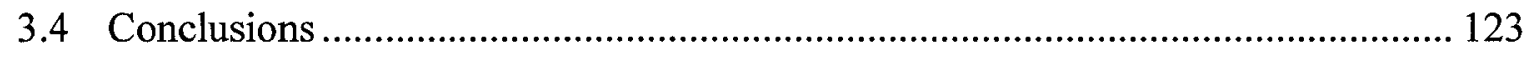

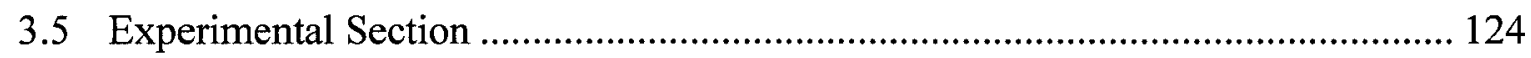

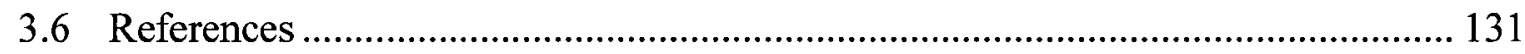

Chapter 4 Electro-Optic Properties of Nonlinear Optical Materials...................... 133

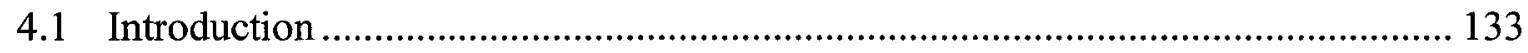

4.2 Electro-Optic Properties of FDCN-Based NLO Polymers ................................... 137

4.2.1 Electro-Optic Properties of FDCN-PES Guest-Host Polymers..................... 137

4.2.2 Electro-Optic Properties of FDCN-MMA NLO Polymers............................ 140

4.3 Electro-Optic Properties of PpQDM-Based NLO Polymers................................... 142

4.3.1 Electric Poling of PpQDM/PVP Guest-Host Polymers............................... 142

4.3.2 Optimization of Poling Conditions................................................................... 143

4.3.3 In situ Poling of PpQDM-PVP Guest-Host Polymers................................... 148

4.3.4 EO Properties of PpQDM-Based Crosslinkable NLO Polyimides ............... 151

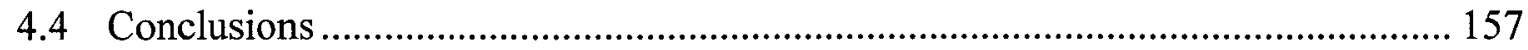

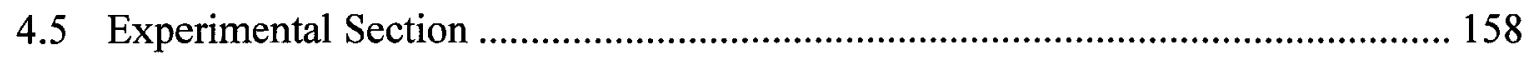

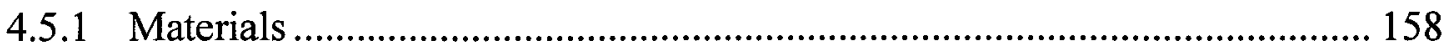

4.5.2 Thin Film Preparation for EO Study ……................................................... 159

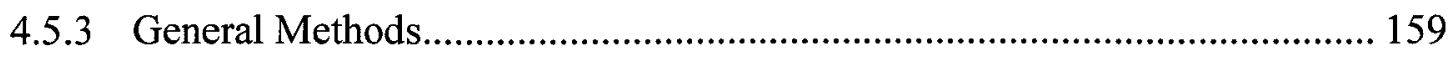

4.5.4 Poling Process and EO Coefficient Measurement....................................... 160 
4.6 References

Chapter 5 Applications of Zwitterionic Chromophores as Near Infrared

Chemosensor

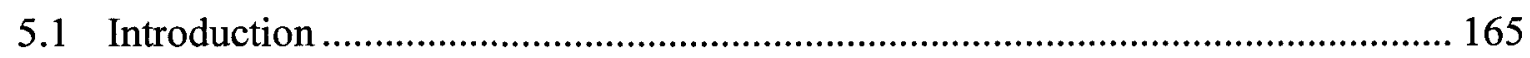

5.2 Influence of Metal ions on the Optical Properties of PpQDM-Met (M1)............. 166

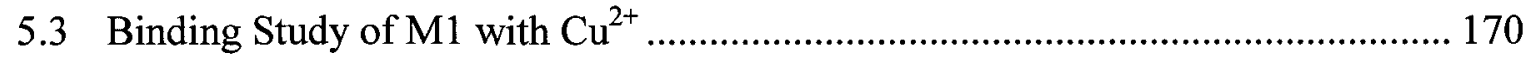

5.4 Formation and Characterization of M1-Cu Complex …..................................... 172

5.5 Design of NIR Fluorescence Turn-On Molecular Probe for Cysteine Detection .. 174

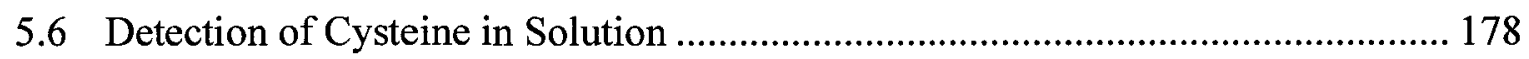

5.6.1 Selective Detection of Cys in the Presence of other Amino Acids .............. 181

5.6.2 Effect of Amines and other Biothiols ........................................................... 184

5.7 Detection of Cysteine in Human Plasma............................................................. 185

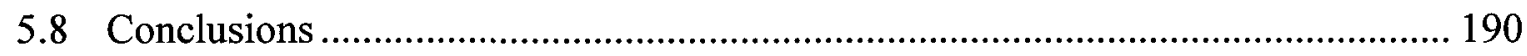

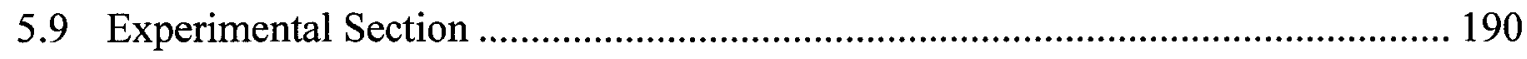

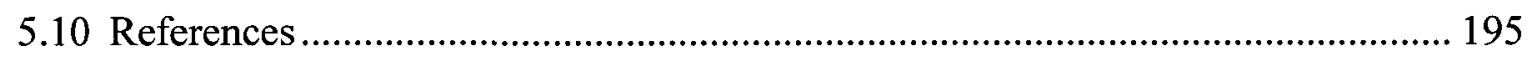

Contribution to Knowledge..................................................................................................... 198

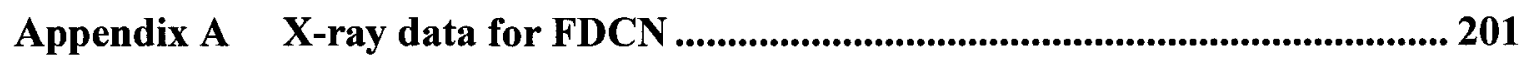

Appendix B Spectra of Synthesized Products of Chapter 2 and 3 ....................... 206

Appendix C Supporting Information of Chapter 4 ................................................ 217

Appendix D Supporting Information of Chapter 5 ................................................... 218 


\section{List of Tables}

Table 1.1. Chromophores contains thiophene and thioazole moieties......................... 13

Table 1.2. Chromophores with modified acceptors .................................................. 14

Table 1.3. Effect of conjugation length on $\mu \beta$ value............................................... 16

Table 1.4. Representative betain dyes and merocyanine dyes................................... 19

Table 1.5. Polyphenylenes bridge /TCF acceptor zwitterionic chromophores ............ 20

Table 1.6. $\mathrm{D}^{+}-\pi-\mathrm{A}^{-}$tertiary amine/3CNQ adducts ............................................... 22

Table 2.1. Selected IR data for 2, FDCN, FCNE ................................................. 57

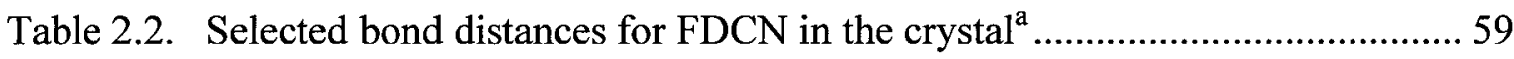

Table 2.3. Selected ${ }^{1}$ H NMR data for FDCN, FCNE, PpQDM-Ben, PeQDM Ben, PpQDM-C3OH, PeQDM-C3OH and PQDM-C3OH ............................ 66

Table 2.4. Optical absorption $\left(\lambda_{\max }, \mathrm{nm}\right)$ data for FDCN and PpQDM chromophores.. 70 Table 2.5. Optical properties of the band of CT of FDCN and PpQDMs in DMF........ 74

Table 2.6. Optical properties of the CT band of FDCN in different solvents............... 75

Table 2.7. Optical properties of the CT band of PpQDM-Met in different solvents ..... 77

Table 2.8. Physical properties of FDCN and PpQDM chromophores......................... 80

Table 3.1. Composition and properties of NA1 NLO polymers.............................. 105

Table 3.2. Composition and properties of NA2 polymers ..................................... 107

Table 3.3. Synthesis and characterization of poly (ether imide)s PI-1 ..................... 113

Table 3.4. Synthesis and characterization of crosslinkable NPI polymers ................ 117

Table 4.1. The molecular properties of FDCN chromophores and EO properties of FDCN doped NLO polymers .......................................................... 139

Table 4.2. Characterization of FDCN-MMA polymers....................................... 141

Table 4.3. Characterization of PpQDM guest-host polymer thin films .................... 142

Table 4.4. EO coefficient (pm/V) of PpQDM-Ben/PVP (5 wt \%) ........................... 149

Table 4.5. Characterization of PpQDM Guest-host polymers under in situ poling..... 149

Table 4.6. Characterization of PpQDM-based crosslinkable NLO polyimide films.... 152

Table 4.7. Characterization of PpQDM NLO polymer /PEDOT: PSS/PVA films ...... 156

Table 5.1. Binding constants of Cys and some amino acids with copper ion.............. 184 


\section{List of Figures}

Figure 1.1. Schematic representation of zwitterionic chromophore......................... 1

Figure 1.2. Relationship between molecular first hyperpolarizability and bond length alternation ................................................................................... 10

Figure 1.3. Ground state resonance forms of donor-acceptor polyene.................... 10

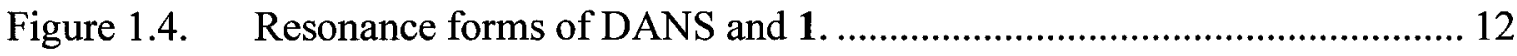

Figure 1.5. Modified electron acceptor structures of TCF class.............................. 15

Figure 1.6. Two neutral benchmark chromophores FTC and CLD........................ 17

Figure 1.7. Zwitterionic chromophores containing heteroaromatic ring in the $\pi$-bridge.

Figure 1.8. TICT and TMC chromophore strucutrs. ......................................... 24

Figure 1.9. A neutral zinc porphyrin complex F10 designed to bind nicotine.......... 34

Figure 1.10. A chemosensing ensemble for histidine........................................ 35

Figure 1.11. Chromophores of the NIR-fluorescent carbocyanine dye ICG (a) and diketopyrrolopyrrole cyanine dyes (b) ........................................... 36

Figure 1.12. Structures of merocyanines I and II. .............................................. 38

Figure 1.13. Contribution of charge-transfer aromatization to ground state of $\mathbf{Q 1}$ and

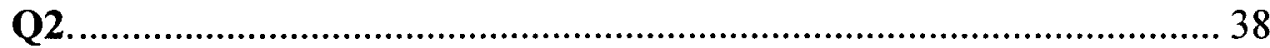

Figure 1.14. Potential modifications of PQDM and two new series of chromophores. 41

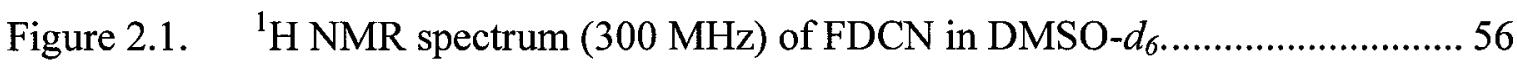

Figure 2.2. IR spectrum of FDCN ( $\mathrm{KBr}$ pellet)............................................... 56

Figure 2.3. ORTEP drawing of the structure of FDCN in the crystal with atomic

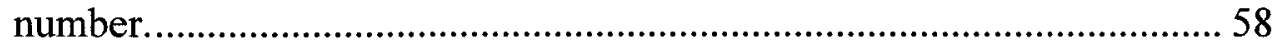

Figure 2.4. Crystal packing diagrams of chromophore FDCN (left); Model for a centrosymmetric dimer of two dipolar dyes (right). .........................6 61

Figure 2.5. TGA (left) and DSC (right) traces of FDCN and BrDCN. .................... 62

Figure 2.6. The formation of PpQDM-C3OH monitored by UV-Vis spectroscopy.... 64

Figure 2.7. IR spectrum of PpQDM-Met (KBr pellet). ...................................6 65

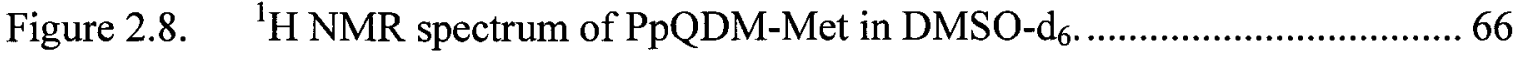


Figure 2.9. TGA trace of $\mathrm{PpQDM}$-Met in nitrogen with a heating rate of $10^{\circ} \mathrm{C} \mathrm{min}^{-1} \ldots$

Figure 2.10. Optical absorption spectra of FDCN (left) and PpQDM (right)

chromophores in DMF solution. 68

Figure 2.11. UV-Vis-NIR spectra of PpQDM-Met in different solvents. 70

Figure 2.12. The normalized absorption and emission spectra of FDCN (left) and PpQDM-Met (right) in DMF. 73

Figure 2.13. Fluorescence spectra of FDCN solutions excited at $460 \mathrm{~nm}$. 76

Figure 2.14. Fluorescence quantum yields $\left(\Phi_{\mathrm{F}}\right)$ of PpQDM-Met vs. the solvent dielectric constant ( $\varepsilon$ ) THF, DCM, acetone, $\mathrm{MeOH}, \mathrm{DMF}, \mathrm{ACN}$ and DMSO 77

Figure 2.15. UV-Vis spectra of PpQDM-Met in $\mathrm{DMF}, \mathrm{H}_{2} \mathrm{O}$ and $\mathrm{HCl}$...................... 82

Figure 2.16. IR spectra of PpQDM-Met and P-PpQDM-Met. ............................... 83

Figure 3.1. $\quad{ }^{1}$ H NMR spectrum of polymer A1-1 ............................................... 103

Figure 3.2. IR spectra of CAEM (left) and polymer A1-1(right)........................ 104

Figure 3.3. IR spectrum of NLO polymer NA1-a. .......................................... 105

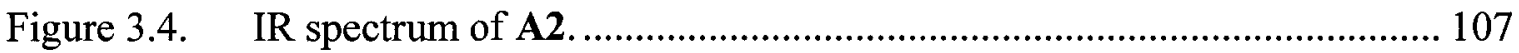

Figure 3.5. UV-Vis spectra of NLO polymers NA2a in DMF and chloroform....... 108

Figure 3.6. Films of PpQDM-Met in different polymers. .................................... 110

Figure 3.7. Absorption spectra of the films of PpQDM-Met in different polymers.. 110

Figure 3.8. TGA (left) and DSC (right) traces of polyimide PI-1b. ..................... 113

Figure 3.9. IR spectrum of NPI-e (KBr pellet). ............................................. 118

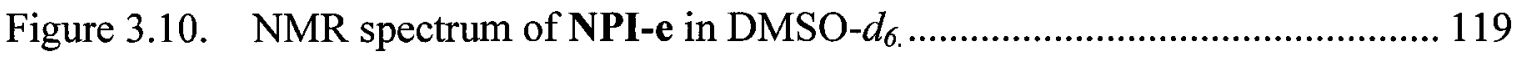

Figure 3.11. UV-Vis absorption spectra in DMF: 1) PpQDM-C3OH 2) NPI-a 3) NPI-b. 120

Figure 3.12. UV-Vis absorption spectra of a solution of PpQDM-C3OH in DMF (1), films of NPI-b (2) and NPI-e (3) ............................................. 121

Figure 3.13. Representative DSC trace of NPI-e with a heating rate of $10^{\circ} \mathrm{Cmin}^{-1} \ldots 122$ Figure 3.14. TGA traces of PQDM-C3OH, NPI-a - NPI-c and NPI-e..................... 122 
Figure 4.1. Schematic diagrams of the corona poling (left) and parallel electrode

poling setup (right).

Figure 4.2. Sandwiched single-layer (left) and double-layer structures (right) for the poling of thin film EO materials.

Figure 4.3. UV-Vis absorption spectra of thin film of PpQDM-Ben /PVP at elevated temperatures in nitrogen

Figure 4.4. Geometries of chromophores: (a) PpQDM-Met (b) PpQDM-Ben ........ 146

Figure 4.5. Temporal stability of the $r_{33}$ for the poled PpQDM-Ben/PVP at $80^{\circ} \mathrm{C} . .148$

Figure 4.6. In situ poling process plot of 5\% PpQDM-Ben/PVP NLO polymer film. (a) Poling temperature (b) Applied poling field (c) EO coefficient value.... 150

Figure 4.7. UV spectra (left) and thermal stability (right) of NLO polyimide NPI-d thin films.

Figure 4.8. UV-Vis-NIR spectra showing optical transparency for PEDOT: PSS/PVA thin films.

Figure 4.9. UV spectra of NPI-d double layer films (left); thermal stability comparison of the NPI-d in the single and double layer films (right)..... 155

Figure 4.10. Schematic diagram of the simple reflective Teng-Man setup 161

Figure 5.1. Fluorescence spectra of $\mathbf{M 1}\left(c=2 \times 10^{-5} \mathrm{M}\right)$ in DMF at various concentrations of $\mathrm{Fe}^{2+}$. The concentrations of $\mathrm{Fe}^{2+}$ are increasing from 0 to $1.6 \times 10^{-4} \mathrm{M}$.

Figure 5.2. Changes in the UV-Vis spectra of $\mathbf{M} 1\left(2 \times 10^{-5} \mathrm{M}\right.$ in DMF $)$ upon titration by $\mathrm{FeCl}_{2}$ from $2 \times 10^{-5}$ to $12 \times 10^{-5} \mathrm{M}$.

Figure 5.3. Fluorescence responses of M1 $\left(c=2 \times 10^{-5} \mathrm{M}\right)$ in the presence of different metal ions $\left(c=2 \times 10^{-4} \mathrm{M}\right)$ in DMF solution. 168

Figure 5.4. Changes in the absorption spectra of M1 in DMF $\left(2 \times 10^{-5} \mathrm{M}\right)$ upon addition of $\mathrm{CuCl}_{2}\left(0\right.$ to $\left.30 \times 10^{-5} \mathrm{M}\right)$.

Figure 5.5. Changes in the fluorescence spectra of M1 in DMF $\left(2 \times 10^{-5} \mathrm{M}\right)$ upon addition of $\mathrm{CuCl}_{2}\left(0\right.$ to $\left.30 \times 10^{-5} \mathrm{M}\right)$.

Figure 5.6. Fluorescence spectra of $\mathbf{M} 1\left(2 \times 10^{-5} \mathrm{M}\right)$ in DMF at various metal ions. .... 
Figure 5.7. Proposed structure of the M1-Cu complex........................................... 173

Figure 5.8. Baseline corrected IR spectra $\left(4000-600 \mathrm{~cm}^{-1}\right)$ of $\mathbf{M 1}$ and M1-Cu........ 174

Figure 5.9. Fluorescence spectra of $\mathbf{M 1 - C u}\left(\mathbf{M 1}=2 \times 10^{-5} \mathrm{M}\right)$ upon addition of Cys $\left(4 \times 10^{-5}-20 \times 10^{-5} \mathrm{M}\right)$ in comparison with the fluorescence spectrum of M1 $\left(2 \times 10^{-5} \mathrm{M}\right)$. Inset: FRP versus the concentration of Cys................ 179

Figure 5.10. Fluorescence intensity of M1-Cu versus Cys concentration. ................. 179

Figure 5.11. Response time measurement for a step change in Cys concentration .... 180

Figure 5.12. Changes in the absorption spectra of M1-Cu $\left(\mathbf{M 1}=2 \times 10^{-5} \mathrm{M}\right)$ upon addition of Cys $\left(4 \times 10^{-5} \mathrm{M}\right.$ to $\left.20 \times 10^{-5} \mathrm{M}\right)$ in comparison with the spectrum of M1 $\left(2 \times 10^{-5} \mathrm{M}\right)$.

Figure 5.13. Changes in absorption (top) and fluorescence spectra (bottom) of M1-Cu $\left(\mathbf{M 1} 2 \times 10^{-5} \mathrm{M}\right)$ in DMF upon addition of $\alpha$-amino acids .

Figure 5.14. Fluorescence recovery percentage (FRP) of M1-Cu $\left(\mathbf{M 1}=2 \times 10^{-5} \mathrm{M}\right)$ towards various natural $\alpha$-amino acids $\left(4 \times 10^{-5} \mathrm{M}\right)$. 183

Figure 5.15. Changes in fluorescence spectra of M1-Cu $\left(\mathbf{M 1}=2 \times 10^{-5} \mathrm{M}\right)$ in DMF with various other chemicals including $\mathrm{L}$-cystine, ethanethiol, ethyl sulfide and propylamine $\left(4 \times 10^{-5} \mathrm{M}\right)$ with and without Cys $\left(4 \times 10^{-5} \mathrm{M}\right)$ in comparison with the fluorescence spectrum of M1 $\left(2 \times 10^{-5} \mathrm{M}\right)$ in DMF. 185

Figure 5.16. Changes in the absorption spectra of $\mathbf{M 1 - C u}\left(\mathbf{M 1}=2 \times 10^{-5} \mathrm{M}\right)$ upon addition of human plasma with Cys (HP-Cys) in concentrations of $2 \times 10^{-5}$ $\mathrm{M}$ to $30 \times 10^{-5} \mathrm{M}$ in comparison with the absorption spectrum of M1 $(2 \times$ $\left.10^{-5} \mathrm{M}\right)$.

Figure 5.17. UV-Vis absorption spectra (left) and fluorescence recovery percentage (right) of M1 $\left(2 \times 10^{-5} \mathrm{M}\right), \mathbf{M 1}-\mathbf{C u}\left(\mathbf{M 1}=2 \times 10^{-5} \mathrm{M}\right)$ and the titration of M1-Cu with deproteinized plasma solutions spiked with various amino acids with or without of Cys. 188 


\section{List of Schemes}

Scheme 1.1. Typical structure of NLO chromophores. ........................................... 4

Scheme 1.2. Schematic representation of a fluorescent chemosensor......................... 30

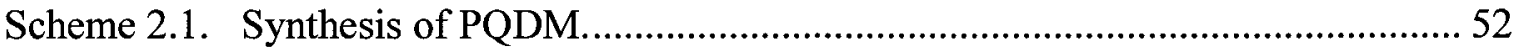

Scheme 2.2. Synthesis of crucial intermediate 2 and FDCN chromophores................. 55

Scheme 2.3. Synthesis of 2-hydroxyethyl cyanoacetate....................................... 55

Scheme 2.4. Resonance structures of Brooker dye and FDCN. ............................... 60

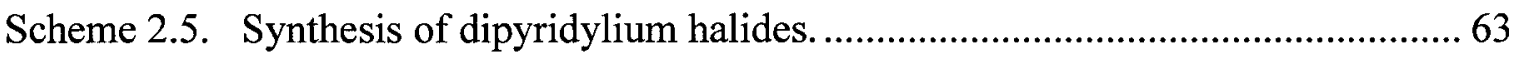

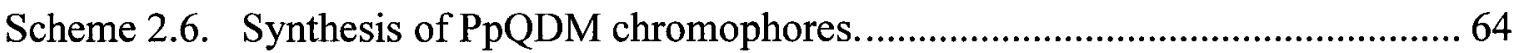

Scheme 2.7. Resonance structures of PpQDM-Met. ........................................ 72

Scheme 2.8. Protonation of PpQDM-Met and deprotonation of P-PpQDM-Met. ........ 82

Scheme 3.1. Synthesis of CAEM and A1 polymers............................................. 102

Scheme 3.2. Synthesis of NLO polymers NA1 ........................................... 104

Scheme 3.3. Synthesis of acrylate FDCN monomer A2 ...................................... 106

Scheme 3.4. Synthesis of NLO polymers NA2 ................................................. 107

Scheme 3.5. Poly(ether imide) PI-1 derived from DAPBA................................... 112

Scheme 3.6. Thermolysis and dimerization of BCBO......................................... 115

Scheme 3.7. Synthesis of NLO polyimides. ..................................................... 116

Scheme 5.1. Conceptual design of colorimetric and NIR fluorescence turn-on probe for detection of cysteine, based on the complexation and de-complexation of NIR fluorophore and copper ion. 


\section{List of Symbols and Abbreviations}

$\beta \quad$ Molecular first hyperpolarizability

$\chi^{(2)} \quad$ Second-order susceptibility

$\varepsilon \quad$ Dielectric constant

$\Phi_{\mathrm{F}} \quad$ Fluorescent quantum yield

$[\eta]_{\text {Inh }} \quad$ Inherent viscosity

$\lambda_{\max } \quad$ Maximum absorption wavelength

$\mu \quad$ Dipole moment, Debye $\left(10^{-18} \mathrm{esu} / \mathrm{cm}\right.$ or $\left.3.336 \times 10^{-30} \mathrm{C} / \mathrm{m}\right)$

$r_{33}$ Electro-optic coefficient in the poling field direction, $\mathrm{pm} / \mathrm{V}$

$\mathrm{T}_{\mathrm{d}} \quad$ Decomposition temperature

$\mathrm{T}_{\mathrm{g}} \quad$ Glass transition temperature

3CNQ Tricyanoquinodimethane

AC Alternate current

ACN Acetonitrile

AIBN Azobisisobutyronitrile

APC Amorphous polycarbonate

BCBO 5-Aminobenzocyclobutenone

BLA Bond-length-alternation

CAEM 2-Cyanoacetoxyethyl methacrylate

CT Charge transfer

Cys Cysteine

DBU 1,8-Diazabicyclo[5,4,0]undec-7-ene

DC Direct current

DCC Dicyclohexylcarbodiimide

DCM Dichlomethane

DEMI 4-[1-Cyano-3-diethylamino-2-propenylidene]-2,5-cyclohexadiene1-ylidenepropanedinitrile

DMAc N, N-dimethylacetamide

DMAP 4-Dimethylaminopyridine

DMF N, N-dimethylformide

DMSO Dimethyl sulfoxide

DSC Differential scanning calorimetry

EDC 1-(3-(Dimethylamino)propyl)-3-ethylcarbodiimide

EO Electro-optic

esu Electrostatic units

FRP Fluorescence recovery percentage

GSH L-glutathione

Hcy Homocysteine 
HOMO Highest occupied molecular orbitals

HRMS High resolution mass spectroscopy

HRS Hyper-Rayleigh scattering

IR Infrared

ITO Indium-tin oxide

LHS Left-hand-site

LTC Leak through current

LUMO Lowest unoccupied molecular orbitals

$n \quad$ Refractive index

$\mathrm{N}$-Acys $\mathrm{N}$-acetylcysteine

NIR Near infrared

NLO Nonlinear optical

NMR Nuclear magnetic resonance

PEDOT Poly(ethylene dioxythiophene)

PES Polyethersulfone

PET Photoinduced electron transfer

PL Photoluminescence

PMMA Poly(methylmethacrylate)

PQDM $\beta$-[1-Substituted-4-pyridinium]- $\alpha$-cyano-4-styryldicyanomethanide (trivial name: pyridinium tricyanoquinonedimethane)

PSS Poly(styrenesulfonic acid)

PVA Poly(vinyl alcohol)

PVP Polyvinylpyrrolidone

RHS Right-hand-site

TCF Tricyanofuran

TCNQ 7,7,8,8-tetracyanoquinodimethane

TGA Thermogravimetric analysis

THF Tetrahydrofuran

TICT Twisted intramolecular charge-transfer

UV-Vis Ultraviolet-visible

Note: Usual abbreviation are used for amino acids 


\section{Chapter 1 Introduction and Background}

\subsection{Introduction}

Zwitterions are molecules, which contain both positive and negative charges at different locations but have an overall net charge of zero. Zwitterionic chromophores are an important type of the intramolecular charge-transfer (ICT) molecules, in which $\pi$ conjugated systems are terminated at opposite ends by donor and acceptor moieties $\left(\mathrm{D}^{\delta+}\right.$ $\pi-\mathrm{A}^{\delta} ; \mathrm{D}$, donor; A, acceptor; $\delta$, degree of $\mathrm{CT}$ ) (Figure 1.1). Zwitterionic chromophores have been investigated for their potential as functional materials for dyes, ${ }^{1}$ nonlinear optics, ${ }^{2}$ photovoltaic ${ }^{3}$ and photochromic ${ }^{4}$ materials, rectifiers, ${ }^{5}$ and conductors. ${ }^{6}$

Many unique and interesting characteristics have been observed for zwitterionic molecules including highly charge-separated ground state, large dipole moment, large negative solvatochromism, and large negative first molecular hyperpolarizabilities $(\beta)$ as well as small energy gap between the highest occupied molecular orbitals (HOMO) and the lowest unoccupied molecular orbitals (LUMO). In the last two decades, zwitterionic chromophores have found successful applications as nonlinear optical (NLO) chromophores and for molecular recognition. ${ }^{7}$

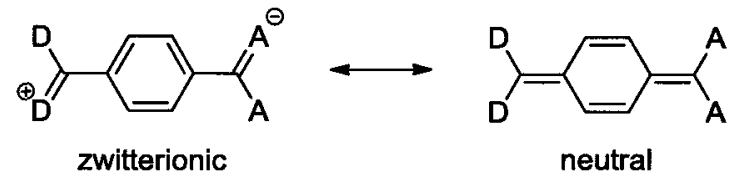

Figure 1.1. Schematic representation of zwitterionic chromophore.

The NLO properties of zwitterionic chromophores and their applications in telecommunications and optical information processing are being actively investigated. Their microscopic NLO efficiencies, characterized by their first molecular 
hyperpolarizabilities $(\beta)$, are related to the electronic ICT excitation between the ground and excited states of the molecules. ${ }^{8}$

The ground state and the first excited state of such molecules can be represented as linear combinations of neutral and zwitterionic limiting forms, and their contributions can be estimated through the use of structural parameters, prominent among which is bond length alternation (BLA), ${ }^{9}$ defined as the difference between the average carboncarbon single and double bond lengths in the polymethine backbone.

According to the "two-state" 10 and the BLA models, the increase of molecular $\beta$ values comes from the decrease of HOMO-LUMO transition energies or reduction in the BLA of the entire conjugated system and the use of suitable electron donor and acceptor groups. These features facilitate electron delocalization therefore resulting in small optical gaps, occurring the near infrared (NIR) region, in zwitterionic chromophores.

Zwitterionic chromophores have been developed as solvent polarity indicators due to the large negative solvatochromic effect seen in solvents of increasing polarity. The longest wavelength ICT absorption band shifts in a wide range from the ultravioletvisible (UV-Vis) to NIR region, but unfortunately they are not fluorescent. ${ }^{11}$ Selective and sensitive NIR fluorescent dyes have attracted much attention in recent years because they offer distinct advantages in biological, clinical, and environmental analyte sensing. However, few zwitterionic chromophores with small band gap have been explored as NIR molecular fluorescent probes.

Distinctive properties of zwitterionic chromophores depend on the subtle interplay between several factors, such as the strength of the end groups and the connectivity of the $\pi$-electron bridge. Understanding the structure and property 
relationships is clearly desirable for the successful design and development of new dipolar zwitterionic chromophores as potential NLO materials and NIR fluorescent probes.

The focus of this research is the development of dipolar zwitterionic chromophores and the corresponding polymers for applications as organic electro-optic (EO) materials and for NIR fluorescent sensing. The first major goal of this research was the synthesis and characterization of two new series of zwitterionic chromophores, dicyanodimethane-based (FDCN) and tricyanoquinodimethane-based (PpQDM) (Chapter 2), their corresponding NLO polymers (Chapter 3), and a study of their intrinsic electric properties. Additionally, the development of an understanding of the structure and property relationships of these zwitterionic chromophores through primary application in EO materials (Chapter 4) as well as a study of the photoluminescence (PL) and NIR chemical sensing properties (Chapter 5) for further successful design of new functional materials. This chapter provides background information on electro-optic materials and fluorescent chemosensors including the rationale for the design of new zwitterionic chromophores.

\subsection{Electro-Optic Effect and Organic EO Materials}

\subsubsection{Basics to Electro-Optic Effect}

NLO effects result from the polarization induced in a material by an intense electric field. Organic second-order NLO materials have attracted much attention in the past two decades due to their potential for use in telecommunications, optical data storage and digital signal processing, optical modulation, and many other applications as active 
materials in photonic microdevices. ${ }^{12}$

The EO or Pockels effect is one of the most important NLO effects, and refers to the change of the optical properties of noncentrosymmetric materials in response to an applied electric field. Through the Pockels effect, the applied field can modulate the amplitude, phase, frequency, or direction of a light beam, which is one of the most widely used mechanisms in high-speed optical modulation and sensing devices, ultra-fast switches, and frequency-doubling devices. For optical communication technologies, EO modulators serve as the key components to encode high-speed electrical signals on an optical carrier wave. ${ }^{13}$ An example of such signal transduction is the encoding of digital electronic computer data onto the optical or photonic internet. Various EO modulators made of inorganic crystals, such as $\mathrm{KH}_{2} \mathrm{PO}_{4}$ and $\mathrm{LiNbO}_{3}$ have been experimentally demonstrated. ${ }^{14}$

In recent years, there has been growing interest in organic EO materials due to their potential applications in telecommunication, digital signal processing, $\mathrm{THz}$ generators, and photonic nano-micro devices. ${ }^{15}$ Organic EO materials consist of many nonlinear optically active molecular units-NLO chromophores. NLO chromophores are molecules with extended $\pi$-electron conjugation system terminated at opposite ends by donor and acceptor moieties, in which the delocalized electron density can be polarized easily in response to an applied electric field (Scheme 1.1). These chromophores may be combined in pure form or dispersed in an optically inactive matrix such as a polymer.

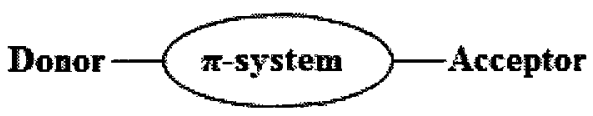

Scheme 1.1. Typical structure of NLO chromophores. 
In the case of an isolated chromophore, the application of a relatively weak electro-magnetic field induces a polarization $(\mathrm{P})$ of electron density that is proportional or linear to the applied field,

$$
P=P_{0}+\alpha_{l} E_{J}
$$

where $P_{0}$ represents the equilibrium polarization, $\alpha_{l}$ denotes a particular tensor element of the linear polarizability, and $E_{j}$ is a vector component of the applied electric field. In bulk materials, the field versus polarization description becomes:

$$
\left.P=P_{0}+\chi^{(l)}{ }_{1 j} E_{J} \quad \text { (eq. } 2\right)
$$

where $\chi^{(l)}{ }_{1 j}$ represents linear susceptibility. When an optical field of a particular frequency interacts linearly with a material, the material exhibits a time varying polarization response proportional to its intensity. This response serves to alter the incident field as to create a new field that is of the same frequency, in which the propagating wave is phaseshifted in time with respect to the incident field. The magnitude of this phase shift is proportional to the index of refraction $(n)$ of the material.

When a sufficiently intense optical field, such as laser radiation, interacts with the material, the description of the response needs to be expanded to include nonlinear (higher order) polarization terms. On the single molecule level and the bulk level, this is described by eq. 3 and eq. 4 .

$$
P=P_{0}+\alpha_{l j} E_{J}+\beta_{l j k} E_{J} E_{k}+\gamma_{y j k l} E_{J} E_{k} E_{l}
$$

where $\beta_{l j k}$ and $\gamma_{l j k l}$ are the frequency dependent first and second molecular hyperpolarizabilities, respectively.

$$
P=P_{0}+\chi^{(1)}{ }_{l j} E_{J}+\chi^{(2)}{ }_{j k} E_{j} E_{k}+\chi^{(3)}{ }_{j k l} E_{j} E_{k} E_{l}
$$


where $\chi^{(2)}{ }_{y k}$ and $\chi^{(3)}{ }_{y k}$ denote the first and second nonlinear susceptibilities, the subscripts $i, j$ and $k$ refer to a molecule-based coordinated system.

Unlike the linear case, time-varying nonlinear polarization effects give rise to new fields of altered frequency with respect to the incident radiation. This phenomenon results in second harmonic generation (SHG) in the case of $\chi^{(2)}{ }_{y k}$ and third harmonic generation (THG) in the case of $\chi^{(3)}{ }_{l j k}$. In addition to frequency alternation in the form of SHG, first nonlinear susceptibility, $\chi^{(2)}{ }_{y k}$, gives rise to an electric field dependent effective combined susceptibility. This accounts for the Pockels effect in which control of electromagnetic susceptibility and refractive index is affected by application of a low-frequency electric field.

In a bulk material, nonzero even-order susceptibility $\chi^{(2)}$ can only come from noncentrosymmetric media. A bulk material consisting of many molecularly asymmetric chromophores, the need for overall noncentrosymmetry imposes the additional requirement of molecular alignment in order to achieve a finite macroscopic second-order nonlinear optical activity. $\chi^{(2)}{ }_{z z z}(\omega)$ (the principle element of the nonlinear susceptibility tensor at frequency $\omega$ is related to the molecular parameters by:

$$
\chi_{z z z}^{(2)}(\omega)=N f \beta(\omega, \varepsilon)<\cos ^{3} \theta>
$$

where $N$ (molecules $/ \mathrm{cm}^{3}$ ) denotes the number density of active molecules that interact with the incident optical field, $\beta_{z z z}$ is the hyperpolarizability tensor element coincident with the molecular symmetry axis, which is dependent on the wavelength (frequency, $\omega$ ) of the incident light field, ${ }^{16}$ and the dielectric permittivity $(\varepsilon)$ of the electro-optic material. ${ }^{17} \theta$ is the angle between the field direction and the $z$-direction. The term of $<\cos ^{3} \theta>$ is the average molecular acentric order parameter denoting the extent of 
chromophore alignment relative to the laboratory $z$-axis. The $z$-axis is parallel to the applied poling field, in electric field poled materials, as well as the field vectors of the optical and electrical operating fields in the case of an EO device. $f$ is the local field factor taking into account the attenuation of applied electric field by the dielectric properties of the host. ${ }^{18}$

The EO effect is an electric field dependent change in refractive index utilized in many photonic device applications including EO modulators for electrical-to-optical signal transduction. EO modulators operate by impressing an electronic signal onto a laser beam, thereby altering the phase and amplitude of the light passing through it; it acts as an ultrafast shutter, blocking and unblocking the laser beam, thereby creating a signal. This control over the phase and frequency of an electromagnetic wave propagating through the material can be expressed by eq. 6 in terms of applied field $(E)$ and refractive index $(n)$.

$$
E=-2 \Delta n / r_{33} n^{3}
$$

where $\Delta n$ is the change in refractive index experienced by the propagating electromagnetic wave and $r_{33}$, the EO coefficient, denotes the magnitude of phase (refractive index, $\Delta n$ ) shift obtained for an applied low-frequency electric field and is given in units of $\mathrm{pm} / \mathrm{V}$. This EO coefficient is determined by the following equation:

$$
r_{33}=-2 \chi_{z z z}^{(2)}(\omega) / n^{4} \quad(\text { eq. } 7)
$$

where the subscript 3 indicates the poling field direction, $z$ is the direction along which the molecular permanent dipole moment occurs and $n$ is the refractive index of the EO material. 
Therefore, the $r_{33}$ of a noncentrosymmetric material can be related to the secondorder susceptibility $\chi^{(2)}$ and the molecular $\beta$ by combination of eq.5 and eq.7.

$$
r_{33}=2<\chi_{222}^{(2)}>/ n^{4}=2 N f<\beta(-\omega ; \omega, 0)>/ n^{4}=2 N f \beta<\cos ^{3} \theta>/ n^{4} \quad \text { (eq.8) }
$$

Equation 8 demonstrates the three most important factors in the optimization of $\chi_{z z z}^{(2)}(\omega)$ and in turn, $r_{33}(\omega)$ : molecular first hyperpolarizability, chromophore number density, and acentric order need to be maximized simultaneously.

In the case of $\pi$-conjugated dipolar molecules, $\beta_{z z z}$ is largely determined by molecular composition and can be tuned using synthetic chemistry; unfortunately, $N$ and $<\cos ^{3} \theta>$ are not independent due to strong inter-chromophore electrostatic interactions. As the density of the highly dipolar chromophores increases, the average distance between them decreases and strong dipole-dipole interactions begin to compete with ordering forces such as the poling field. Therefore, the loading parameter $N\left(<\cos ^{3} \theta>\right)$ must be optimized using molecular engineering approaches to control chromophore shape, lattice attachment geometry, solubility, etc. ${ }^{19}$

\subsubsection{Molecular Hyperpolarizability}

The first molecular hyperpolarizability, $\beta_{z z z}(\omega, \varepsilon)$ of dipolar NLO chromophores relies on the ground state electron density distribution asymmetry, which can be tuned through the asymmetric substitution of a $\pi$-electron-conjugated bridge with the appropriate donor and acceptor moieties. According to the simple two-state theoretical model by Oudar and Chemla, ${ }^{20}$ the molecular $\beta$ can be expressed as:

$$
\beta_{z z z} \propto \frac{\Delta \mu_{e g}\left(\mu_{g e}\right)^{2}}{E_{g e}{ }^{2}}
$$


where $\Delta \mu_{\text {eg }}$ represents the difference in dipole moment between the HOMO and the LUMO; $\mu_{\mathrm{ge}}$ represents the transition dipole moment (oscillator strength) and $E_{\mathrm{ge}}$ represents the energy difference between ground (HOMO) and charge transfer excited states (LUMO).

Typically, stronger electron-density donors and acceptors as well as a longer conjugated bridging unit lead to a larger $\beta_{z z z}(\omega, \varepsilon)$. This rule works well for chromophores consisting of an aromatic bridge that partially energetically impedes polarization. However, the use of powerful donor/acceptor pairs or a particular conjugated interconnect, such as polyphenylene, does not necessarily lead to the realization of optimal $\beta$ values, an optimal combination of donor and acceptor strengths has to be matched for maximization of the $\mu \beta$ value. ${ }^{21}$

This behaviour may be understood by another theory in terms of BLA, proposed by Gorman and Marder, ${ }^{9}$ which correlates $\beta$ with the degree of charge separation in the ground state (Figure 1.2) and in turn is dependent on the chemical structure and environment of the chromophore, due to the induced ground state polarization. ${ }^{22}$ For dipolar molecules, BLA can vary from positive to zero and to negative, depending on the relative contributions of neutral and charge-separated resonance form to the ground state, which in turn depends on the donor and acceptor strengths. For the given donor and acceptor groups, the magnitude of BLA depends on the transition energy, which is dependent on the nature and the length of the $\pi$-conjugated bridge. ${ }^{23}$ 


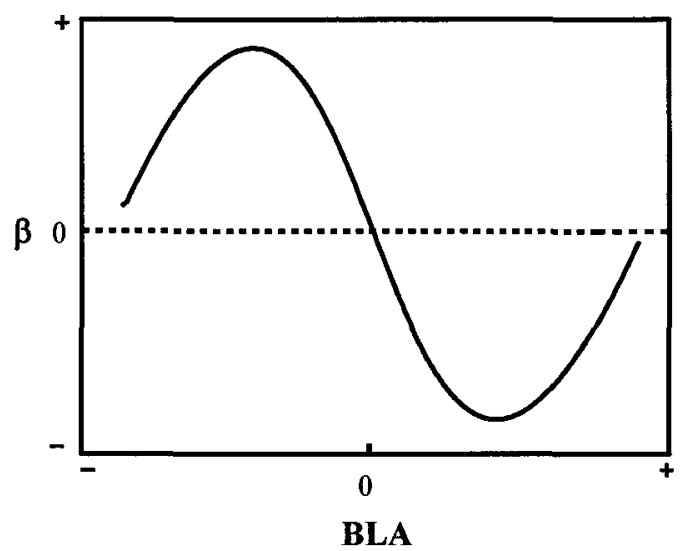

Figure 1.2. Relationship between molecular first hyperpolarizability and bond length alternation.

Two maximum $\beta$ vales were predicted by BLA theory. A positive BLA value characterizes a neutral state where as a charge-separated (or zwitterionic) state is characterized by a negative BLA value. The negative sign of $\beta$ for zwitterionic molecules indicates that the direction of ICT in these molecules is opposite to that in neutral molecules. If both resonances have an equal contribution, no BLA is observed and $\beta$ becomes zero; this situation is referred to as the cyanine limit (Figure 1.3).

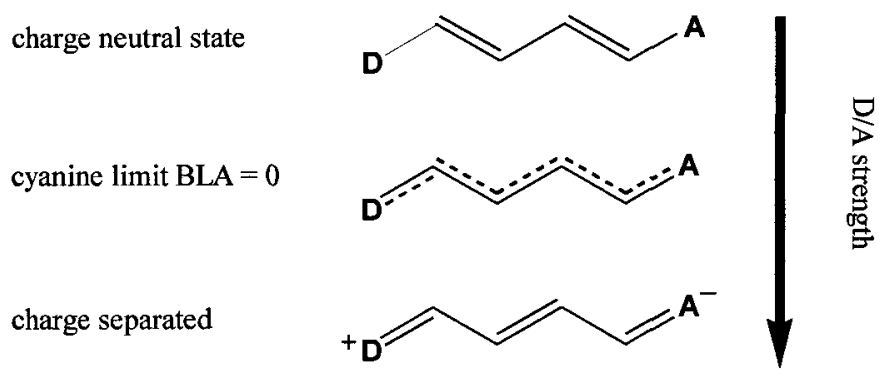

Figure 1.3. Ground state resonance forms of donor-acceptor polyene.

A large positive BLA and a small positive $\beta$ value result for a chromophore which has weak donor and acceptor groups. In this case the neutral state dominates the ground state; upon increasing the strength of the donor and acceptor, the contribution of the 
charge-separated state increases, resulting in a decreased BLA which passes through zero. As the strength of the donor and acceptor continue to increase eventually the chargeseparated ground state dominates, leading to a reverse pattern with a negative BLA and a negative $\beta$ value. Therefore, two local maximum $\beta$ values can be found, in the positive region for neutral chromophores ${ }^{24}$, and in the negative region for zwitterionic chromophores. $^{25}$

\subsubsection{Neutral Nonlinear Optical Chromophores}

The past three decades have seen a large library of second order NLO chromophores developed and characterized with initial focus on simple para-substituted stilbenes (e.g., DANS in Figure 1.4). ${ }^{26}$ These early systems did not lead to the expected high EO active materials; until 1990's, the BLA theory supported by the two-state model gave insight into the structure property relationship for both neutral and zwitterionic NLO chromophores. As a result, careful selection of donor, acceptor and conjugated bridge is crucial for a maximized molecular hyperpolarizability of a molecule.

\subsubsection{Electron Donors}

A large number donor groups capable of contributing electron density have been explored over the past decade. Although sulphur and oxygen based donors have also been explored, ${ }^{27}$ the most successful type donor structure falls into the general class of alkyl and aryl amines as a result of their strong electron-donating properties and thermal stability. ${ }^{28}$ Although no great difference between the alkyl and aryl amines has been reported, studies have indicated that a larger $\mu \beta$ value could result by using diarylamine than by using dialkylamine. ${ }^{29}$ In the correct geometry, the energy level of the nitrogen 
nonbonding lone pair is well matched to donate into the $\pi^{*}$ orbital of the attached aromatic ring. This arrangement destabilizes the HOMO and reduces ionization potential creating a strong electron density donating effect. However, the degree of ground state polarization is not sufficient to maximize the $\beta$ value due to the loss of the aromaticity in the charge-separated form upon polarization.

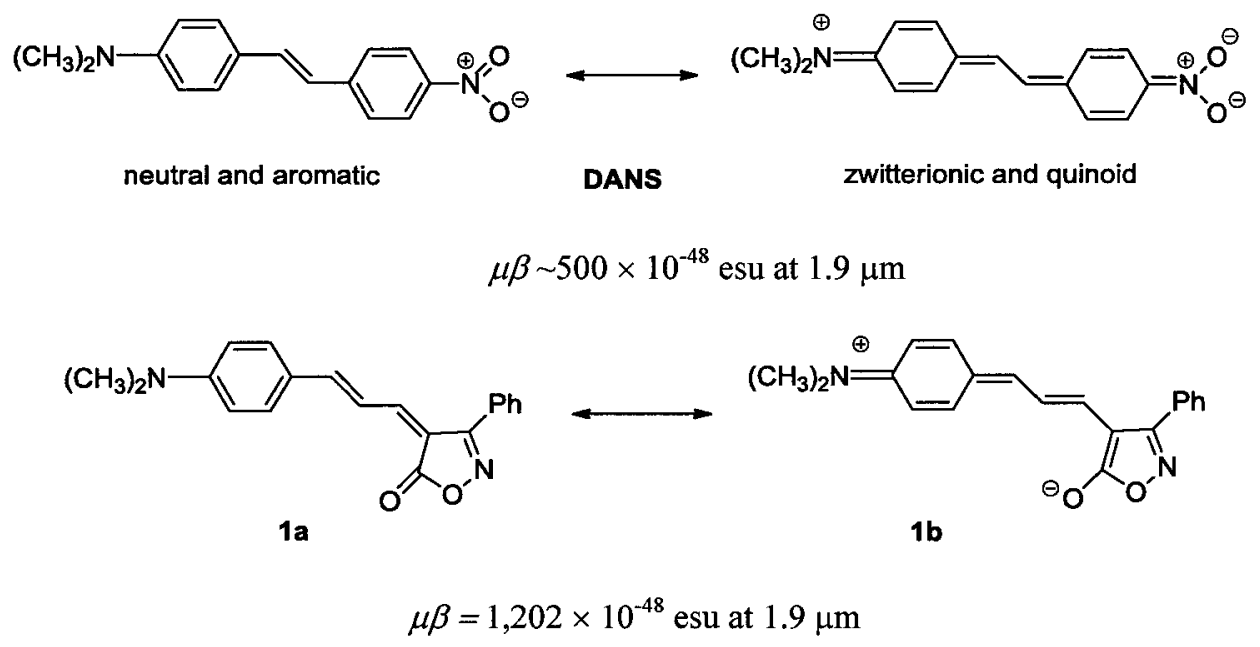

Figure 1.4. Resonance forms of DANS and 1.

An effective strategy to solve this problem was proposed in designing new dipolar chromophores with aromatic acceptors, in which the loss of aromatic stabilization in one part would be compensated by a gain in aromaticity in another part. In addition, this strategy can result in an increased ground state dipole moment $(\mu)$. For example, 3phenyl-5-isoxazolone was introduced as the acceptor to chromophore $1,{ }^{4 a}$ the $\mu \beta$ value of $\mathbf{1}$ became much higher than that of DANS, which is similar in conjugation length to 1 (Figure 1.4).

Another approach to improve the $\mu \beta$ value is replacement of benzene rings in the $\pi$-bridge with heteroaromatics such as thiophene ${ }^{30}(2$, Table 1.1$)$ and thioazole ${ }^{31}(4$, 
Table 1.1). These heterocyclic rings have lower aromaticity energy than that of benzene, and as a result offer more contributions to the ground state, reduce the BLA and improve the thermostability of these chromophores.

Table 1.1. Chromophores contains thiophene and thioazole moieties

\begin{tabular}{|c|c|c|c|}
\hline Name & Structure & $\begin{array}{c}\mu \beta\left(\times 10^{-48} \mathrm{esu}\right) \\
\text { at } 1.9 \mu \mathrm{m}\end{array}$ & Ref. \\
\hline 2 & & 1040 & 30 \\
\hline 3 & & 800 & 31 \\
\hline 4 & & 2190 & 31 \\
\hline
\end{tabular}

As shown in Table 1.1 , by replacing the benzene ring with thiophene, the $\mu \beta$ value increases from $500 \times 10^{-48}$ esu for DANS to $1040 \times 10^{-48}$ esu for chromophore 2; and replacing the benzene ring with thioazole, the $\mu \beta$ value increases from $800 \times 10^{-48}$ esu for 3 to $2190 \times 10^{-48}$ esu for chromophore 4 . Both designs proved efficient in increasing the $\mu \beta$ values.

\subsubsection{Electron Acceptors}

Like electron donors, a large number of electron accepting groups with diverse chemical structures have been prepared over the past decade. More recently, the tricyanofuran-based (TCF) acceptor family, were developed and tested to be most promising due to the high chemical, thermal, and photostability with strong electron affinity. $^{32}$ 
Table 1.2. Chromophores with modified acceptors

NTC Structure $\begin{gathered}\mu \beta\left(\times 10^{-48} \text { esu }\right) \\ \text { at } 1.9 \mu \mathrm{m}\end{gathered}$

Table 1.2 shows the examples $(\mathbf{5}, \mathbf{6}$ and FTC) that have primarily the same donor and $\pi$-conjugate bridge but different acceptor groups. Increases in the $\mu \beta$ values of about 10 times are observed upon changing the dicyanovinyl electron acceptor for $5^{33}$ to more efficient TCF electron acceptor for FTC. ${ }^{34}$

Following the success of such materials based on the unfunctionalized TCF acceptor, ${ }^{35}$ significant effort has been focused toward chemical modifications that improve electron affinity, allow secondary functionalization, or modify the shape of the chromophore. Several modified TCF structures are shown in Figure 1.5. Simple TCF (A1) is illustrated for comparison. In simple chromophores, switching the acceptor from TCF to CF3-TCF (A3) may significantly reduce the optical band gap $\Delta E_{\mathrm{ge}}$ by as much as $0.3-$ $0.5 \mathrm{eV}$ and roughly double the $\beta$ value. ${ }^{36}$ 
<smiles>CC1=C(C#N)C(C)(C)OC1=C(C#N)C#N</smiles>

A1<smiles>CCN1C(=O)C(=C2OC(C)(C)C(C)=C2C#N)C(=O)N(CC)C1=S</smiles>

A2<smiles>CC1=C(C#N)C(=C(C#N)C#N)OC1(C)C(F)(F)F</smiles>

A3<smiles>CC1=C(C#N)C(=C(C#N)C#N)OC1(c1ccccc1)c1ccccc1</smiles>

A4

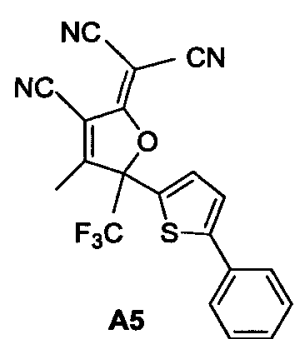

Figure 1.5. Modified electron acceptor structures of TCF class.

\subsubsection{Bridges and Chromophores}

A number of parameters must be satisfied when selecting a suitable conjugated bridge, including thermal and chemical stability, electronic character and maximizing $\beta$. Generally, an increase in conjugation length leads to an increase in observable molecular hyperpolarizabilty as seen in chromophores 7-10 (Table 1.3). For a given donor-acceptor chromophore, upon addition of one ethylene unit in the $\pi$-bridge, $\mu \beta$ value increases from $6700 \times 10^{-48}$ esu for 7 to $9800 \times 10^{-48}$ esu for $8^{37}$ A doubling of $\mu \beta$ value for 9 was observed upon insertion of each additional ethylene unit into the $\pi$-bridge. ${ }^{38}$ However, increasing the polyene length does not represent a practical approach to optimization of the molecular $\mu \beta$ value. First, beyond certain conjugation lengths the polarization saturates. Additionally, since the absorption show a bathochromic shift with increasing the length of conjugation, absorbance in the telecommunications wavelengths becomes an unfavourable possibility. Furthermore, increases in conjugation length can also lead to lowered chemical and thermal stabilities. ${ }^{39}$ 
Table 1.3. Effect of conjugation length on $\mu \beta$ value

Name $\begin{gathered}\mu \beta\left(\times 10^{-48} \text { esu) }\right. \\ \text { at } 1.9 \mu \mathrm{m}\end{gathered} \quad$ Ref.

Incorporation of aromatic bridges usually leads to good thermal and photochemical stability due to the aromaticity of the ring lowers the energy of the HOMO consequently reducing the susceptibility of the chromophore to oxidation and chemical reaction; however, hyperpolarizability is reduced compared with the use of a polyene bridge. A compromise has been reached by using heterocyclics such as thiophenes, furans and pyroles, which offer lower delocalization energies when compared to aromatic rings and also better thermal stabilities when compared to the polyenes. ${ }^{40}$

Successful design is represented by the FTC (Figure 1.6, left), ${ }^{41}$ thiophene-based bridges offer an attractive compromise between large $\beta$ value and stability under a wide range of chemical synthesis conditions. 

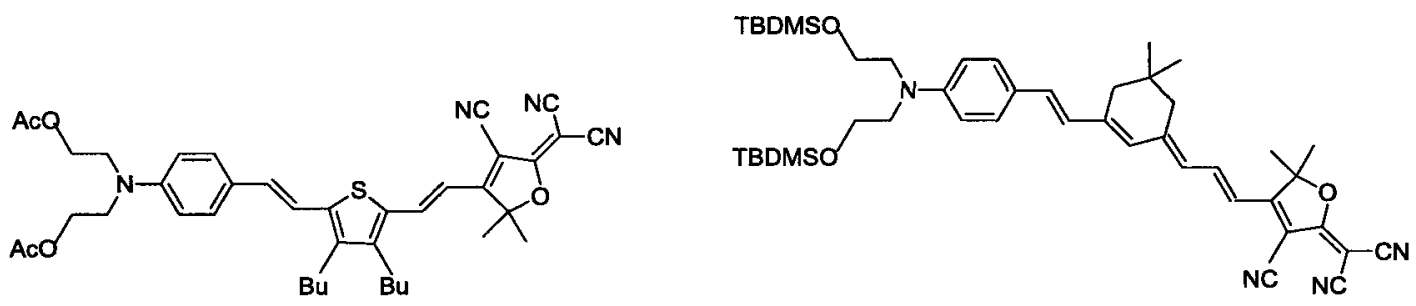

$$
\begin{gathered}
\text { FTC }(\mu=12 \mathrm{D}) \\
\mu \beta=17,600 \times 10^{-48} \text { esu at } 1.9 \mu \mathrm{m} \\
r_{33}=55 \mathrm{pm} / \mathrm{V}(20 \mathrm{wt} \% \text { in PMMA })
\end{gathered}
$$

$$
\begin{gathered}
\mathrm{CLD}(\mu=13 \mathrm{D}) \\
\mu \beta=14,065 \times 10^{-48} \text { esu at } 1.9 \mu \mathrm{m} \\
r_{33}=92 \mathrm{pm} / \mathrm{V}(25 \mathrm{wt} \% \text { in } \mathrm{APC})
\end{gathered}
$$

Figure 1.6. Two neutral benchmark chromophores FTC and CLD.

Recently, sterically protected (ring-locked) polyenes were successfully exploited in the $\pi$-bridging structures to increase the conjugation length and to produce high- $\mu \beta$ chromophores with good thermal stability. ${ }^{42,43}$ A typical example is CLD, developed by Dalton and coworkers (Figure 1.6), in which the ring locked olefinic moiety is incorporated in the polyenes bridge to improve chemical and thermal stabilities, enforce a trans conformation of the polyene to increase an effective conjugation, and to change the shape of the chromophore reducing intermolecular interactions. ${ }^{24}$

FTC and CLD-type chromophores are two standard neutral chromophores, which have shown significant enhancement of the molecular $\mu \beta$ values (greater than $15000 \times$ $10^{-48}$ esu at $\left.1.9 \mu \mathrm{m}\right)$ and good thermostability has been demonstrated through optimization of the conjugation bridge structure and donor/acceptor pairing.

\subsubsection{Zwitterionic Nonlinear Optical Chromophores}

According to Marder's plot (Figure 1.2), two maxima of the $\beta$ occur on either side of the cyanine limit, a positive $\beta$ on the left-hand-site (LHS) refers to chromophores with neutral ground states, and a negative $\beta$ on the right-hand-site (RHS) refers to 
chromophores with zwitterionic ground states. Even though the absolute value of $\beta$ is the only consideration, NLO chromophore synthesis has focused predominantly on traditional neutral push-pull systems, and little attention has been paid to zwitterionic chromophores. Zwitterionic dyes were developed initially as solvent polarity indicators due to their large negative solvatochromism. Compounds exhibiting solvatochromism are also of potential interest as materials that may exhibit the NLO property under the appropriate conditions. Recently, several zwitterionic dyes have found successful application as NLO chromophores due to their extremely large $\mu \beta$ values. ${ }^{2 \mathrm{c}}$ Betterdesigned zwitterionic chromophores are developing since their electronic and structural properties are of considerable interest for new applications.

According to structure, some representative zwitterionic chromophores were classified and shown in Table 1.4 to Table 1.6. The charge-separated ground states of these chromophores are inferable from their strong negative solvatochromism and were demonstrated by the X-ray crystallography and theoretical modeling. ${ }^{44,45}$

\subsubsection{Betain Dyes and Merocyanine Dye}

The first class is Betain dyes $(\mathbf{1 1}, \mathbf{1 2})$ and merocyanine dyes $(\mathbf{1 3}, \mathbf{1 4})$, which both show characteristically large negative solvatochromism (Table 1.4). Solvatochromism is the ability of a chromophore to exhibit a change in colour as a result of a change in solvent polarity. The absorption shifts results from a difference of dipole moments in ground and excited states of the chromophore. Negative solvatochromism refers to a hypsochomic (blue) shift occurring when the chromophore has a charge-separated ground state. Positive solvatochromism refers to a bathochromic (red) shift when the chromophore has a neutral ground state. In the case of negative solvatochromism, an 
increase in solvent polarity stabilizes the ground state of zwitterionic chromophores, and an increase in the energy gap between the polar ground and non-polar excited states occur. This energy change in turn affects the absorbance and emission position as well as the shape of the resulting UV/Vis/NIR and PL spectra.

Table 1.4. Representative betain dyes and merocyanine dyes

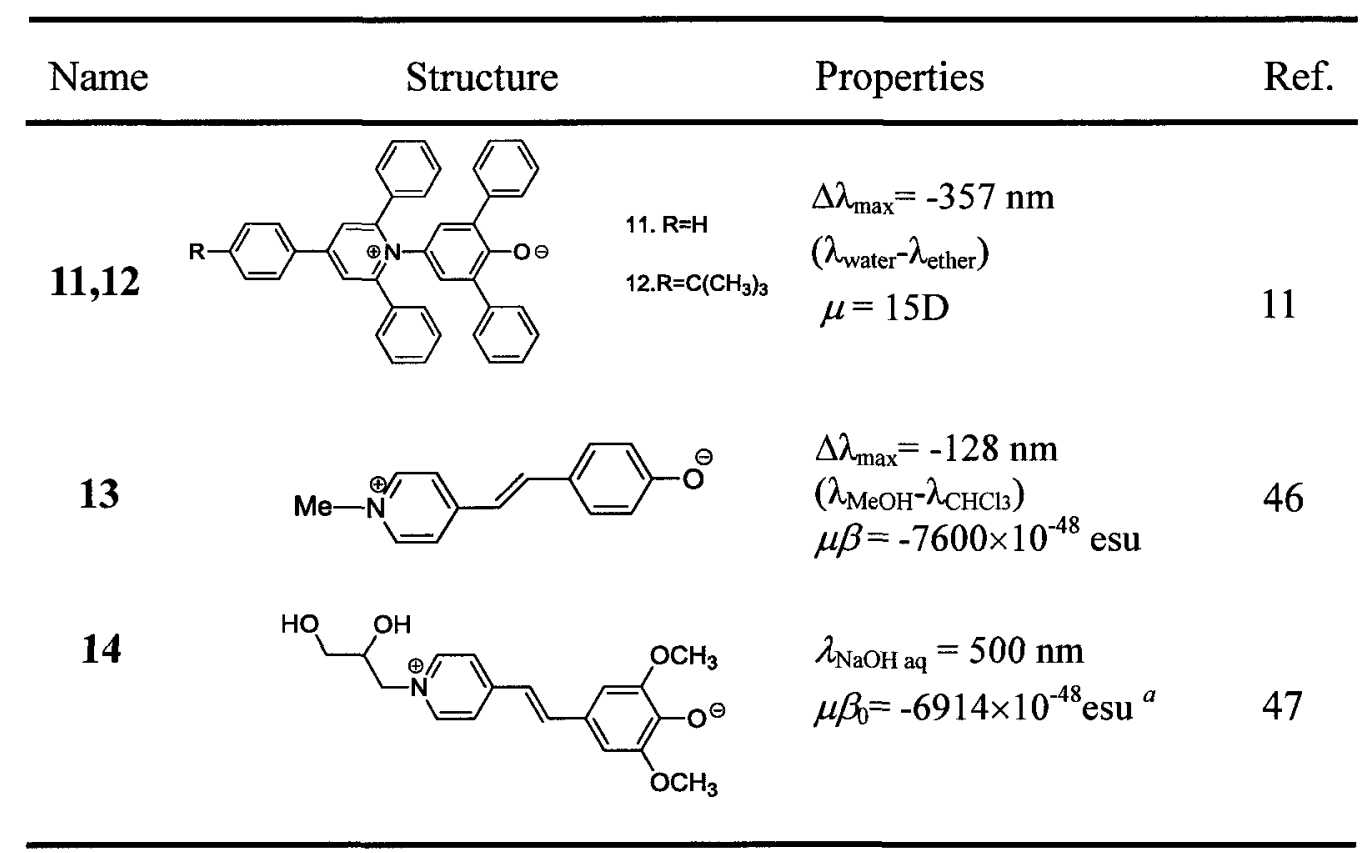

${ }^{a} \beta_{0}$ : zero-frequency molecular first hyperpolarizability

In particular, 11 and its more lipophilic derivative 12 (soluble even in hydrocarbons) are the basis of the $\mathrm{E}_{\mathrm{T}}{ }^{(30)}$ scale. Upon excitation, electron transfer occurs from the oxygen atom to the center of the aromatic system. The dipole moment is about $15 \mathrm{D}$ in the ground state whereas it is nearly zero in the excited state. ${ }^{11}$ The longest wavelength ICT absorption band is hypsochromically shifted by $357 \mathrm{~nm}$ on going from diphenyl ether $\left(\lambda_{\max }=810 \mathrm{~nm}\right)$ to water $\left(\lambda_{\max }=453 \mathrm{~nm}\right)$. 


\subsubsection{Polyphenylenes Bridge/Tricyanofuran-Based Acceptor}

The second class is phenylpolyenes end-capped with aromatic donor and the strong tricyanofuran-based (TCF) acceptor groups $(15,16)$, which exhibit large $\mu \beta$ values through complicated syntheses (Table 1.5).

Table 1.5. Zwitterionic chromophores containing polyphenylenes bridge /TCF acceptor

Name 15 Ref. $\begin{aligned} & \text { Properties } \\ & \mu \beta_{0}\left(\times 10^{-48} \mathrm{esu}\right)\end{aligned}$

\subsubsection{Heteroaromatic Ring Containing $\pi$-Bridge}

The third class are molecules containing heteroaromatics (e.g., thiophene) in the $\pi$-bridge, whose reduced ring aromaticity relative to benzene leads both to an increased transition moment and to a change in dipole moment (Figure 1.7). ${ }^{48}$ 


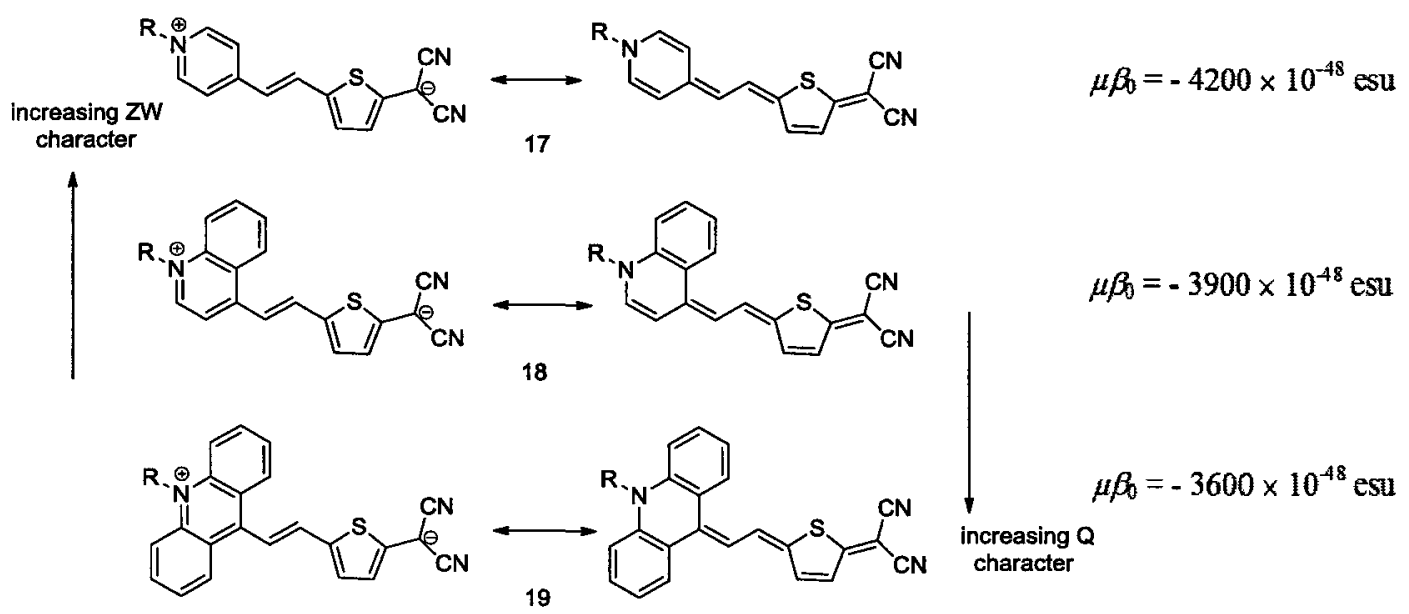

Figure 1.7. Zwitterionic chromophores containing heteroaromatic ring in the $\pi$-bridge.

In comparison with neutral chromophores of comparable sizes (e.g. 5: $\mu \beta_{1.9 \mu \mathrm{m}}=$ $1300 \times 10^{-48} \mathrm{esu}$ ), zwitterionic chromophores (17-19) containing thiophene in the bridge exhibit much larger $\mu \beta$ value and the increased dipole moment (e.g., 17: $\mu=20 \mathrm{D}$ in dioxane; 7: $\mu=6 \mathrm{D}$ )

Also the $\mu \beta$ values are dramatically influenced by large orders of magnitude, by a consecutive annelation of the pyridyl ring of these chromophores (pyridine-quinolineacridine) and medium polarity. In polar media, the ground states of these chromophores can be described as linear combinations of zwitterionic and quinoid forms. Figure 1.7 clearly shows that, the importance of the zwitterionic form sequentially decreases, and the quinoid form simultaneously increases, ongoing from pyridine 17 to quinolone 18 to acridine 19. The main reason is that the pyridine ring loses aromaticity in the right-hand side formula and the acridinium ring can better sustain a quinoid character due to the restored full aromaticity of the two benzene side rings. In less polar media, negative $\beta$ values were obtained only in 17 , and become positive and increase from 18 to 19 . The 
annelation effect explains the reasons behind why quinolone and acridine rings have hardly been used in designing new dyes with optimized $\beta$ values.

\subsubsection{4 $\mathrm{D}^{+}-\pi-\mathrm{A}^{-}$Tertiary Amine/ 3CNQ-Based Zwitterionic Chromophores}

The fourth class are $\mathrm{D}^{+}-\pi-\mathrm{A}^{-}$tertiary amine/ tricyanoquinodimethane-based (3CNQ) zwitterionic chromophores, which have been extensively studied of their NLO properties (Table 1.6).

Table $1.6 . \mathrm{D}^{+}-\pi-\mathrm{A}^{-}$tertiary amine/3CNQ adducts

Name

$3 \mathrm{CNQ}$ is one of the best acceptors known to date, where the gain in aromatic stability from a one electron reduction is the large driving force behind this powerful acceptor. The 3CNQ acceptor is based on the electron acceptor 7,7,8,8tetracyanoquinodimethane (TCNQ), which was first reported in $1960^{50}$ and is well known to form semi-conducting salts since TCNQ readily accepts an electron to form stable anion radicals. TCNQ has also been shown to react with tertiary amines such as triethyl amine and 2- or 4-methylquinoline to form stable zwitterionic molecules, DEMI and 
PQDM, respectively. These TCNQ derivations have also shown multifunctional properties as molecular rectification $^{5 \mathrm{a}}$ and photochromic behaviour. ${ }^{51}$

DEMI has displayed very large $\mu \beta$ value at zero frequency when compared to push-pull systems of similar length due to the aromatic stability gained by the $3 \mathrm{CNQ}$ acceptor when in the charge-separated state. Due to the low requirement for extensive conjugations in yielding large $\mu \beta$ values, zwitterionic chromophores are typically easier to be prepared than neutral chromophores.

However, despite the large $\mu \beta$ values and ease in preparation, DEMI suffers from very low solubility due to its very planar structure, and a strong tendency to form antiparallel aggregates. Furthermore, the olefinic interconnect is very sensitive to both heat and light. To be usable in practical EO applications, the donor moiety of this class of zwitterionic chromophores must be optimized to meet the required properties. Considering the pyridium-based analogue PQDM, although no $\mu \beta$ value were reported, comparative studies by Cross and co-workers ${ }^{52}$ indicated a larger $\beta_{0}\left(-1270 \times 10^{-30} \mathrm{esu}\right)$ for PQDM than that for DEMI $\left(\beta_{0}=-350 \times 10^{-30}\right.$ esu) and predicted a large EO coefficient will be realized in a suitable polymer host.

Most recently, Marks' group $^{2 c}$ reported the development of TMC twisted intramolecular charge transfer (TICT) chromophores consisting of a conjugated donorbridge-acceptor $\pi$-electron system, which provides rotation about the CT axis. Unlike the planar $\pi$-electron system of most dipolar zwitterionic chromophores, the $\pi$-electron conjugation is broken in the twisted biaryl molecules. Theoretical studies have predicted that the degree of rotation modulates the $\pi$-orbital overlap resulting in electric field induced nonlinear polarization is maximized at an optimum dihedral angle of $\theta \approx 70^{\circ}-85^{\circ}$. 
TICT chromophores reported so far consist of substituted biaryl compounds characterized by charge-separated ground states (Figure 1.8), whose calculated $\beta$ and $\mu \beta$ values $\left(\sim 70,000 \times 10^{-48} \mathrm{esu}\right)$ can be two orders of magnitude larger than that of DANS.

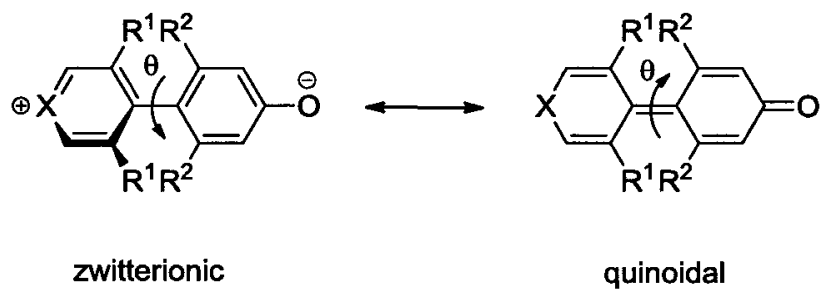

$\mathrm{X}=\mathrm{O}, \mathrm{NCH}_{3} \quad$ TICT Chromophores
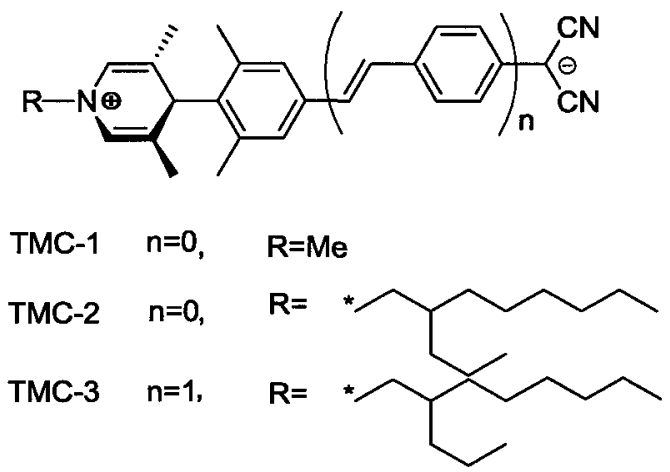

Figure 1.8. TICT and TMC chromophore strucutrs.

The new generation TMC chromophores have large twist angles and exhibit extremely large $\mu \beta$ value. For example, the twist angle $(\theta)$ in TMC- 2 is $89.6^{\circ}$ determined by X-ray diffraction. The hyperpolarizability and dipole moment for extended conjugated molecule TMC-3 was measured as $\mu \beta=-488,000 \times 10^{-48} \operatorname{esu}\left(\lambda_{\exp }=1.907 \mu \mathrm{m}\right)$ using the electric field induced second harmonic generation method (EFISH), which is much larger than that of CLD $\left(\mu \beta=35,000 \times 10^{-48} \mathrm{esu}, \mathrm{EFISH}, \lambda_{\exp }=1.907 \mu \mathrm{m}\right)$.

Unlike the conventional planar chromophores, TICT chromophores with large optical nonlinearity can be simple compounds consisting of two substituted arene rings 
such as TMC-1. Therefore, they exhibit good thermostability and enhance the resistance to photochemical/oxidative degradation. TMC-3 possesses a ground state dipole moment as large as $50.6 \mathrm{D}$ (calculated by DFT method). This large dipole moment, combined with the reduced solubility typically displayed by zwitterionic chromophores, causes this type of chromophore to aggregate aggressively. EO coefficient of TMC-3 was measured to be $330 \mathrm{pm} / \mathrm{V}$ in a polar polyvinylphenol host, using the Teng-Man technique under an applied poling field of $100 \mathrm{~V} / \mu \mathrm{m}$. However, this extraordinarily high $r_{33}$ value has so far only been measured by in situ (constant poling bias) methods. ${ }^{4 c}$

To reduce aggregation, two strategies must be taken into account in preparing EO materials containing TICT chromophores either by synthetic modification of the TICT molecule or by choosing an appropriate polymer hosts.

\subsubsection{Development of Nonlinear Optical Polymers}

Polymers containing NLO active chromophores have emerged as a promising class of EO materials. ${ }^{53,54}$ These materials are typically made from organic chromophores with high microscopic molecular hyperpolarizabilities, incorporated into polymer matrixes, and poled with an external applied electric field at or near the glass transition temperature $\left(T_{g}\right)$ to achieve a noncentrosymmetric dipole alignment.

The polymeric NLO materials have received increasing interests in EO applications because of their high NLO susceptibility, low dielectric constant, fast response time, small dispersion in refractive index, structural flexibility, and ease of material processing. The most striking advantage of poled polymers results from their unique EO mechanism that arises mainly from electronic excitations in individual 
nonlinear molecules by the displacement of electrons not ions, as in inorganic materials, so polymer can provide a much large bandwidth in high frequency operations. ${ }^{54}$

The development of NLO polymers depends on the successful incorporation of the NLO chromophores into a polymer matrix, the poled polymers can be classified into two systems: 1) guest-host systems, where the NLO chromophores (guests) are doped into a polymer (host), 2) substituted systems, where the organic chromophores are covalently bound to a polymer backbone.

\subsubsection{Guest-Host Polymers}

Guest-host systems are the simplest approach to screen NLO chromophores and EO materials, where the chromophores are physically incorporated into a polymer host. They facilitate the combination of different polymer matrixes and chromophores without any complicated chemistry, but suffer from several drawbacks including 1) low concentration of chromophores that can be doped in the polymer without aggregation. Aggregation changes the spectroscopic properties, increases the scattering losses, and limits the mobility of the chromophores, hence, usually reducing the EO response of the system; 2) low temporal stability of the orientation. In general, the stability depends strongly on the local environment provided by the polymer matrix. For practical application, the $\mathrm{T}_{\mathrm{g}}$ of a guest-host NLO polymer should be at least $100{ }^{\circ} \mathrm{C}$ higher than the ultimate operating temperature. Recently, a few thermally stable guest-host systems were successfully established by doping NLO chromophores into higher glass transition temperature polymers, such as amorphous polycarbonate (APC), polyethersulfone (PES), polyquinolines and polyimide. ${ }^{55,56}$ 


\subsubsection{Side-Chain and Crosslinkable NLO Polymers}

The most traditional way to increase chromophore concentration and the temporal stability of the orientation is to covalently attach the chromophores onto the polymers. Side-chain NLO polymers where the chromophore is attached as a side-chain to the backbone, and main-chain NLO polymers where the chromophore is incorporated into the backbone itself are two examples. Main-chain NLO polymers receive relatively little attention because the preparation highly depends on the purity of chromophorecontaining monomer, also the expected large dipole moment cannot be obtained due to the chain entanglements ${ }^{57}$, and poling is much more difficult due to the hard large segmental motions of the polymer backbones.

Compared to main-chain NLO polymers, side-chain polymers represent a more versatile approach to chromophore-linked systems. First, there is significant flexibility in optimization of properties for side-chain NLO polymers through modification of backbone structures, adding side groups, and introduction of crosslinking functionalities. Second, the additional distance that separates the chromophores from the backbones due to the pendent nature of these systems allows for better alignment during the poling process. The restricted mobility improves the stability and helps to reach higher concentrations without aggregation. Furthermore, side-chain NLO polymers can be synthesized either by copolymerizing chromophore-containing monomers or through a post-functionalization, which allows various types of NLO chromophores can be grafted into the polymer under mild conditions, and for the preparation of host polymer capable of undergoing further modifications. 
Good thermal stability of poling-induced dipole order of side-chain NLO polymers can be achieved by grafting NLO chromophores into high- $\mathrm{T}_{\mathrm{g}}$ polymers such as polyurethane, ${ }^{58}$ and polyimide..$^{59}$

Polyimides are the most promising high $\mathrm{T}_{\mathrm{g}}$ polymers for EO study due to their good mechanical strength, low optical loss, good compatibility with highly polar NLO chromophores, (e.g., PQDM) ${ }^{60}$ and can be easily modified by using different polymerizing monomers. The preparation of NLO polyimides typically involves various post-functionalizations and addition polymerization. ${ }^{61}$ First side-chain NLO polyimide was prepared by condensation of hydroxyl-functionalized polyimide with the acidcontaining CLD chromophore. ${ }^{62}$ A high chromophore loading (25 wt \%) and a high EO coefficient $(71 \mathrm{pm} / \mathrm{V})$ at $1.3 \mu \mathrm{m}$ were achieved.

Another way to improve the stability is crosslinking. The crosslinking can be done either before or after the orientation process. However, reduced EO activity was often observed because that spontaneous crosslinking and poling process often proceed together making it difficult to align the dipolar chromophores efficiently. Recently, Jen's group demonstrated an effective strategy to improve the poling efficiency by employing a new cross-linkable system based on the thermally reversible Diels-Alder reaction, the polymer crosslinking while cooling after the poling process. A good temporal stability ( $\sim 80 \%$ of the original $r_{33}$ value) of the cross-linkable NLO polymer was obtained. ${ }^{63}$ 


\subsection{Chemosensor Chemistry and Near Infrared Fluorescent Sensors}

\subsubsection{Basics to Chemosensor}

Selective and sensitive chemosensors are molecules specifically designed for the qualitative and quantitative monitoring of analytes. A chemosensor can be regarded as "a receptor that interacts with an analyte producing a detectable change in a signal" ${ }^{64}$ The changes in physical properties of a chemosensor such as absorption and/or fluorescence spectra have been widely used in biological and analytical chemistry, in medicine and environmental sciences. These types of sensors are extensively used for the analysis of cations, anions and even molecules due to fast and convenient detection. Although electrochemical techniques have also been used, ${ }^{65}$ this short introduction will focus on optical sensors since they will be mainly studied in the next chapters.

The most common types of chemosensors consist of three components for molecular recognition and signal transduction: a receptor (responsible for the selective analyte binding), an active signalling unit (whose properties should change upon the formation of a complex, e.g., fluorescence can be either quenched or enhanced based on molecule or ion recognition) and a spacer that can change the geometry of the system and tune the electronic interaction between these moieties. ${ }^{66}$

\subsubsection{Fluorescent Molecular Sensors of Cations}

Fluorescent molecular sensors are molecules that undergo a change in fluorescent property, intensity or color, upon recognition of target molecules or experiencing an environmental change. Among the versatile chemosensors, fluorescent molecular sensors have distinct advantages for chemosensing and bioimaging due to their high sensitivity, 
selectivity, short response time, local observation (e.g. by fluorescence imaging spectroscopy), low cost and easy fabrication. Moreover, remote sensing is possible by using optical fibers (Scheme 1.2). ${ }^{67}$

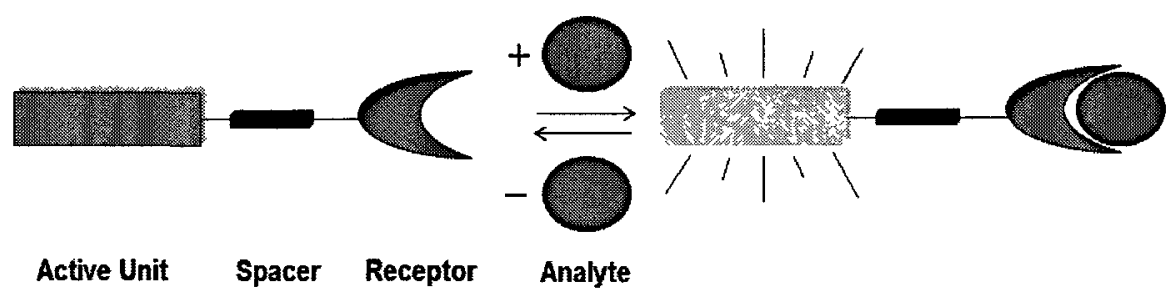

Scheme 1.2. Schematic representation of a fluorescent chemosensor.

The development of fluorescent molecular sensors for metal ions, especially for cations with biological interest such as $\mathrm{K}^{+}, \mathrm{Ca}^{2+}, \mathrm{Cu}^{2+}$, etc. has always been of particular interest, since some of them are present in biological systems in trace amounts and as environmental pollutes in uncontrolled amounts. Additionally, organometallic complexes were used to construct indicator displacement assays (IDAs) for the detection of peptides, amino acids and nucleotides. ${ }^{68}$

In the design of fluorescent molecular sensors for metal ions, special requirements should be met in order to fulfil the criteria of affinity and selectivity. In the design of the metal binding unit (fluoroionophore), the most critical factor is the characteristics of the ionophore moiety i.e. the ligand topology and the number and nature of the complexing heteroatoms or groups, which should match the characteristics of the cation, such as ionic diameter, charge density, coordination number, intrinsic nature (soft, hard, etc.). The considerable changes in the fluorescence of a fluorophore depends on the interaction of cations and ionophores, photoinduced processes including electron transfer (PET), charge transfer (PCT), energy transfer, excimer formation, etc. 


\subsubsection{Fluorescent Molecular Sensors with Open-Chain Receptor}

Receptor containing only one aliphatic amine group<smiles></smiles>

$\mathrm{F} 1 \mathrm{n}=\mathbf{2}$<smiles>CCCCCCCCCCNc1ccc2c3c(cccc13)C(=O)N(CCN)C2=O</smiles>

F2

$F 1^{69}$ and $F 2$ are the simplest molecules appending the fluorophore to the amine by an alkyl chain. A significant fluorescence enhancement (30 times for F1) can be observed in acetonitrile solutions upon metal complexation with many transition metal ions, including those ions that usually quench fluorescence, such as $\mathrm{Fe}^{3+}, \mathrm{Co}^{2+}, \mathrm{Ni}^{2+}$ and $\mathrm{Cu}^{2+}$. The proposed mechanism is that complexation prevents the PET to occur, and that leads to an enhancement of the fluorescence. $\mathbf{F} 2$ was reported ${ }^{70}$ to have a similar effect, but with a greater selectivity for $\mathrm{Cu}^{2+}$, where the fluorescence of the chemosensors $(10 \mathrm{mM})$ is only by much higher concentrations (10-100 ppm) of $\mathrm{Ni}^{2+}, \mathrm{Mn}^{2+}, \mathrm{Zn}^{2+}$ and $\mathrm{Ca}^{2+}$.

\section{Chemosensors composed of polyamine and achelator receptors}

The $\mathrm{pH}$ dependence of the fluorescence intensity changes in the presence of $\mathrm{Cu}^{2+}$, $\mathrm{Ni}^{2+}$ and $\mathrm{Zn}^{2+}$ in acetonitrile/water solutions of $\mathbf{F 3}$ which composed of polyamine receptors and an anthracene fragment was reported. ${ }^{71} \mathrm{~A}$ decrease of the intensity is observed at lower $\mathrm{pH}$ for copper ions, since metal ion complexation in these conditions leads to the deactivation of the excited state via an energy transfer process from the chromophore to metal centred states. 

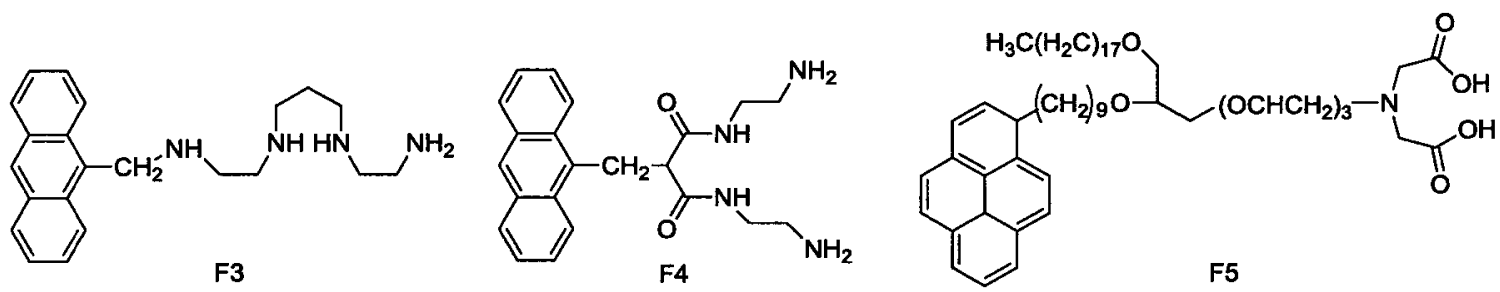

A well-known category of ligands, dioxo-tetraamines were reported to signal the presence of $\mathrm{Ni}^{2+}$ and $\mathrm{Cu}^{2+}$, when connected to chromophores such as anthracene (F4). ${ }^{72}$ The complexation mechanism involves the deprotonation of the two amide groups. This process can occur only with $\mathrm{Ni}^{2+}$ and $\mathrm{Cu}^{2+}$ ions, which profit by a large ligand field stabilization. $\mathbf{F 5}$ is a representative sensor containing chelators with carboxylic groups ${ }^{73}$, it is known to efficiently bind divalent hard metal ions like $\mathrm{Ca}^{2+}$ and $\mathrm{Mg}^{2+}$, but show a remarkably high affinity for $\mathrm{Cu}^{2+}$. Complexation with copper is fast and reversible, and a detection limit of $5 \mathrm{nM}$ for $\mathrm{Cu}^{2+}$ was reported.

\subsubsection{Fluorescent Molecular Sensors with Cyclic Receptor}

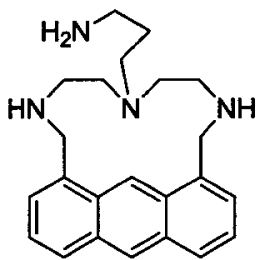

F6

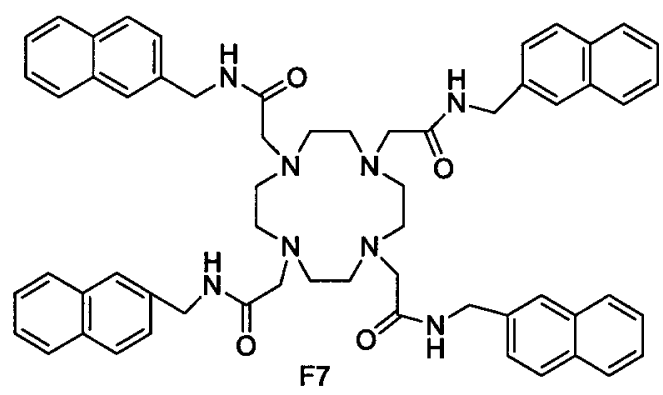

F7
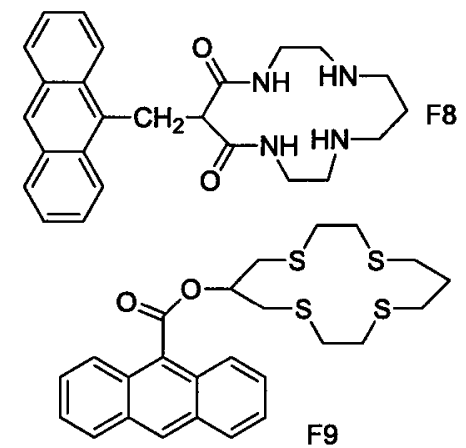

F6 ${ }^{74}$ shows a very high selectivity towards $\mathrm{Cu}^{2+}$ and $\mathrm{Hg}^{2+}$. In water solution at $\mathrm{pH} 7$, this compound displayed chelation-enhanced quenching effects with only these two ions, with overall emission changes of 18 -fold and four fold, respectively. F7 ${ }^{75,76}$ is based on the cyclen receptor and on the naphthalene chromophore. $\mathrm{Cu}^{2+}$ ions leads to a fluorescence decrease of $95 \%$ by the heavy atom effect. $\mathbf{F 8},{ }^{72}$ possessing a dioxocyclam 
receptor shows a similar behaviour to the open-chain analogue $\mathbf{F 4}$, complexing both $\mathrm{Cu}^{2+}$ and $\mathrm{Ni}^{2+}$ ions, and exhibiting the same qualitative behaviour. However, the $\mathrm{pH}$ interval available for selective titration (in which the two ions can be discriminated) is reduced due to the macrocyclic effect. The tetrathia-macrocycle F9, ${ }^{77}$ contains a crown which does not contain nitrogen atoms but four sulfur atoms and is known for its strong affinity towards $\mathrm{Cu}^{2+}$ in the presence of other $3 \mathrm{~d}$ metal ions. Copper binding leads to an efficient quenching of the chromophore luminescence centred at $470 \mathrm{~nm}$ through PET from anthracene to the metal ion. However, $\mathrm{Ag}^{+}$ions are shown to compete in ethanol with $\mathrm{Cu}^{2+}$ ions, forming a stable complex.

\subsubsection{Organometallic Complex-Based Optical Chemosensors}

\section{Intrinsic chemosensors}

These sensors feature the receptor unit as part of the chromophore/fluorophore. ${ }^{66}$ The interaction between the bound substrate and the dye (chromophore or fluorophore) leads directly to the modification of its emission properties.

A typical example of such sensor developed by D'Souza et al., the neutral zinc porphyrin (F10), which was tested as a fluorescencent sensor for nicotine (Figure 1.9). ${ }^{78}$ The molecular recognition of F10 upon binding nicotine in toluene solution was observed by both UV-Vis and fluorescence spectroscopy. The result implied that the pyridyl nitrogen of nicotine axially ligates the zinc ion and the pyrrolidine nitrogen hydrogen bonding to the carboxylic acid group. A bathochromic shift of visible bands of F10 and a decrease in the intensity of the emission bands at $605 \mathrm{~nm}$ and $650 \mathrm{~nm}$ were observed. 


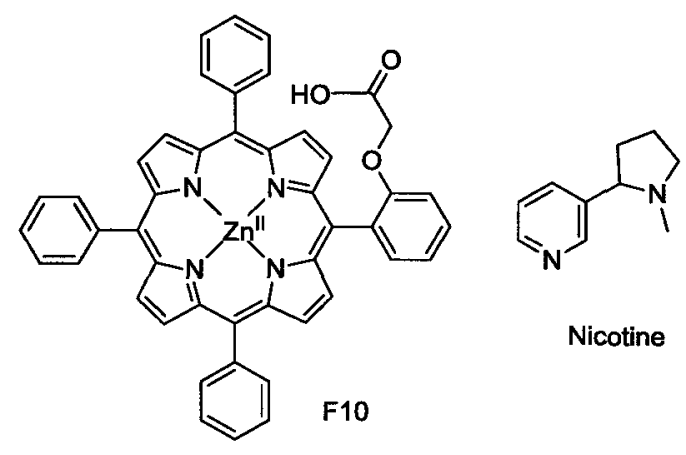

Figure 1.9. A neutral zinc porphyrin complex F10 designed to bind nicotine.

This kind of chemosensor is relatively easy to make, but the development and optimization of these sensors has been proven difficult, because they are intrinsically rigid as they have to be designed around the substrate, any modification of the binding site to modify its selectivity or affinity may change the emission properties of the dye.

\section{Self-Assembled Chemosensors}

The chemosensing ensemble is an easy and convenient strategy that has been developed only in last ten years based on a competition assay. In a metal-based indicatordisplacement assay, the receptor is composed from a metal ion or ions coordinated by ligands. A chromophore/fluorophore is introduced into the system as an indicator to coordinate with the metal center and, eventually, the ligand(s), which results in optical changes that can be measured to derive the binding affinity. Zinc (II), copper (II) and rhodium ${ }^{79}$ complexes have been widely used for this approach and can operate in highly polar solvents (aqueous ethanol or pure water) as well as display strong affinity towards analytes.

This strategy was applied initially by the Fabbrizzi's group for the detection of histidine in water. ${ }^{80}$ Receptor F11 was designed to incorporate two copper ions in order to gain affinity for analytes (Figure 1.10). Eosine was one of the fluorescent indicators 
with emission wavelengths in the visible region, and has a carboxylate group in the molecular structure. Eosine is bound through noncovalent interactions to F11, which quenches its emission; the added analytes displace the Eosine indicator, which was released to the solution restoring its fluorescence. Results showed that F11 binds to histidine better than glycine, phenylalanine, valine, leucine, and proline due to histidine possessing an imidazole residue which coordinates to dicopper centres resulting in higher selectivity.

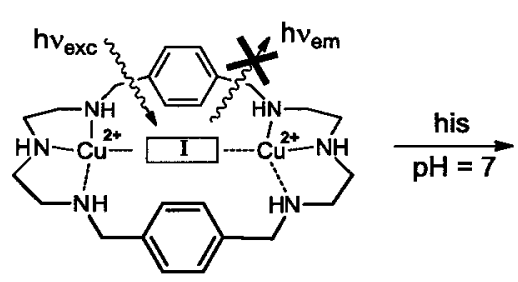

F11-Indicator

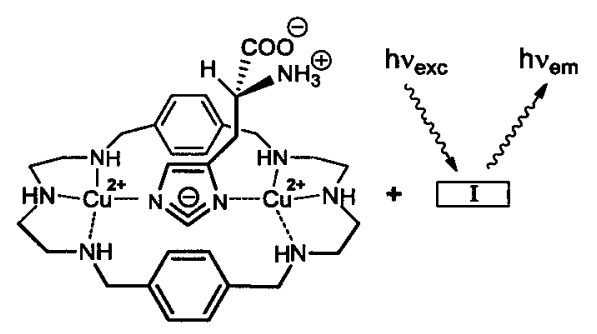<smiles>NC(=O)c1ccccc1-c1c2cc(Br)c(=O)c(Br)c-2oc2c(Br)c(Br)c([N+](=O)[O-])cc12</smiles>

Eosine Indicator

Figure 1.10. A chemosensing ensemble for histidine.

\subsubsection{NIR Fluorescent Molecular Sensors}

Although visible fluorescent molecules play important roles in various research areas, optical imaging of molecular processes in living organisms has stimulated interest in the development of molecular probes for use in the NIR region (typically 700-900 nm). In this spectral window, many intrinsic tissue chromophores and macromolecules have low light absorption, low auto fluorescence and low light scattering. In addition, the NIR light can penetrate much deeper into tissue, which is suitable for therapeutic applications ${ }^{81}$ and can be used as tags or stain for bio-imaging. ${ }^{82}$ 


\subsubsection{NIR Fluorescent Cyanine Dyes}

New NIR imaging methods and molecular probes (Figure 1.11) have received considerable interests and development in the last decade. The dye indocyanine green (ICG) is a gold standard for in vivo optical imaging due to its excellent NIR emission properties and suitability for use in humans. Several ICG derivatives have also been prepared for subsequent conjugation with peptides, antibodies, and other biologically relevant molecules. ${ }^{83}$ However, a major problem with these probes is the occasional lag time between uptake in target tissue and clearance from surrounding tissue.

(a)

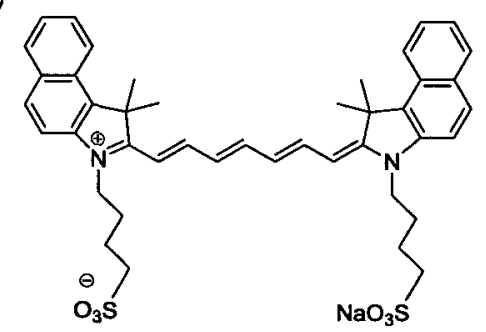

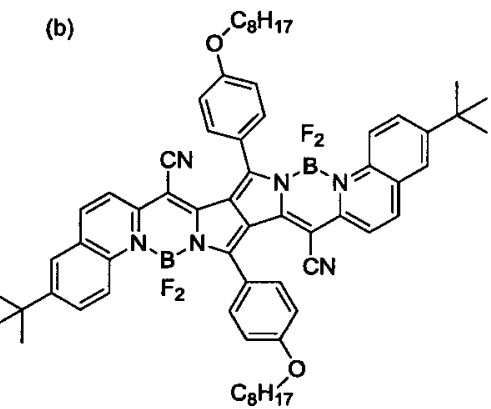

Figure 1.11. Chromophores of the NIR-fluorescent carbocyanine dye ICG (a) and diketopyrrolopyrrole cyanine dyes (b)

Development of active NIR probes, which only emit fluorescence in response to a specific molecular event is necessary. However, earlier polymer-based responsive probes have shown limited access to intracellular enzymes and the slow enzyme-dependent fluorescent enhancement. ${ }^{84}$ Therefore, development of small molecular NIR probes with small HOMO-LUMO gaps for biochemical analytes (amino acids, coenzymes, carbohydrates, etc.) is clearly desirable. 


\subsubsection{NIR Zwitterionic Fluorophores}

The optical and electronic properties of organic molecules are determined by their energy gap, which is the energy difference between the HOMO and LUMO. Narrowing this energy gap is an effective way to obtain a red shift in absorption and emission spectra. Common strategies to reduce the energy gap include 1) extending the conjugation length, 2) introduction of a strong electron donor and a strong electron acceptor, 3) lowering the bond length alternation of $\pi$-conjugated chromophores. ${ }^{85}$

Approaches 1) and 2) have been extensively investigated to tailor the optoelectronic properties of monomeric chromophores in a D- $\pi$-A system. However, the extent of $\pi$-electron delocalization in polyene-type polymers is limited by distortion of the polymer backbones that results in finite energy separations between the valence molecular orbitals. The disadvantages are extensive tedious synthesis for screening new electron donors and acceptors. Recently, adjusting the BLA of zwitterionic chromophores has become a promising strategy to recognize small optical band gap.

In 2006, two hyperpolarizable merocyanines (I and II) showing the strongest PL emission bands at $565 \mathrm{~nm}$ and $640 \mathrm{~nm}$ in 9:1 ethanol-methanol solvent was first reported by Smith's group (Figure 1.12). ${ }^{86}$ More recently, Marks' group ${ }^{4 c}$ reported their studies on the TICT-TMC chromophore aggregation in solution by optical absorption and fluorescence spectroscopies (Figure 1.8). Although TMC-3 has the longest conjugated bridge in the new twisted zwitterionic chromophores, the maximum emission at $485 \mathrm{~nm}$ in $\mathrm{CH}_{2} \mathrm{Cl}_{2}$ is still in the visible region. Moreover, the fluorescent sensitivity of these dyes to solvent polarity is not reported yet. 

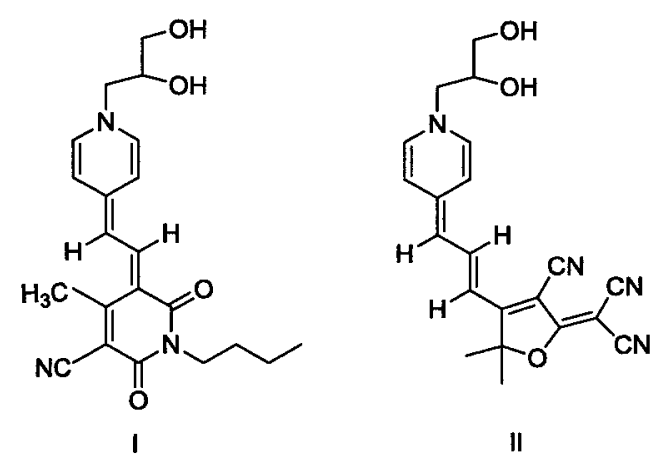

Figure 1.12. Structures of merocyanines I and II.

In 2010, novel donor/ acceptor substituted cross-conjugated carbocycles based on quinoids $(\mathbf{Q 1})$ or expanded quinoids $(\mathbf{Q 2})$ were prepared for reducing HOMO-LUMO gaps. X-ray diffraction shows that these push-pull quinoids have significant chargeseparated ground states (Figure 1.13). The maximum absorption of $\mathbf{Q 1}$ is around $695 \mathrm{~nm}$; extending the conjugation by an additional quinodimethane ring gives Q2 two major absorptions, one is located in the visible region $\left(\lambda_{\max }=610 \mathrm{~nm}\right)$, and the other broad absorption is located in the NIR region $\left(\lambda_{\max }=845 \mathrm{~nm}\right){ }^{87}$ However, the fluorescent property of these new zwitterionic chromophores was not reported.
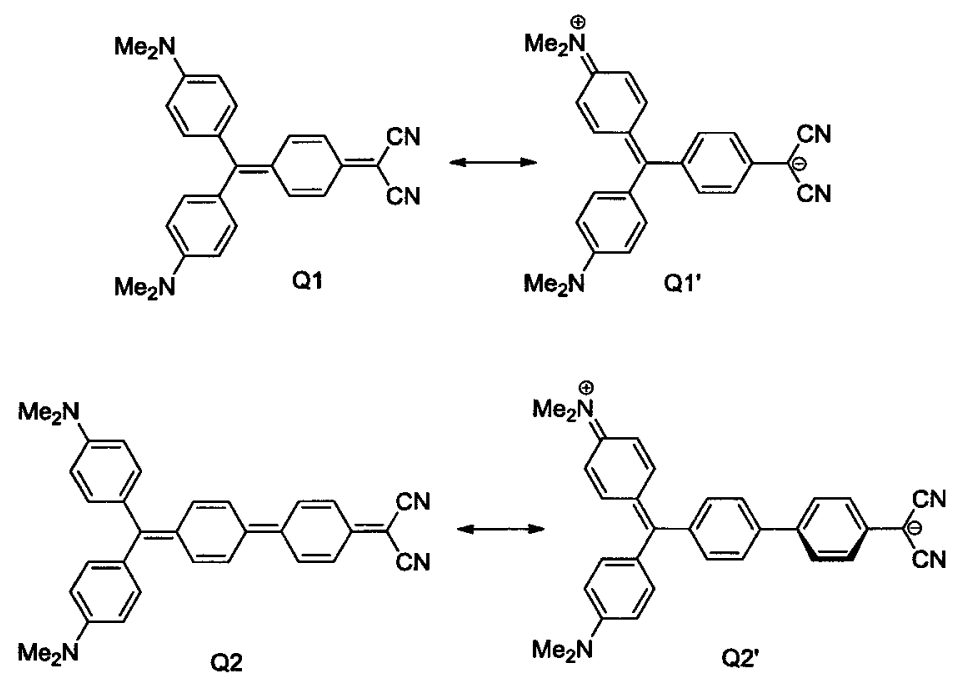

Figure 1.13. Contribution of charge-transfer aromatization to ground state of $\mathbf{Q 1}$ and $\mathbf{Q 2}$. 
$\mathrm{D}^{+}-\pi$ - $\mathrm{A}^{-}$type of zwitterionic dyes derived from a tertiary amine and TCNQ ${ }^{3,4 b}$ show great potential to be NIR fluorophores because they possess a low-energy resonance structure of quinoid having the effectively reduced BLA. In addition, the hybridization of the energy levels of the donor and acceptor also gives rise to a smaller HOMO-LUMO gap. With the similar structures, PeQDM was found recently by our group to exhibit NIR PL $\left(\lambda_{\max }=850 \mathrm{~nm}\right)$ and have potential application for $\mathrm{pH}$ indicator. $^{88}$

\subsection{Rationale and Objectives}

The field of NLO materials for EO applications and chemosensors has been rapidly growing over the last years.

A wide range of NLO chromophores with very large $\mu \beta$ values has recently been prepared according to the established theories, especially for the synthesis and functionalization of neutral chromophores. However, among many requirements for practical EO devices, high macroscopic nonlinearity, which requires a high microscopic molecular nonlinearity of NLO chromophores and an efficient dipole alignment induced by applying an electric field, is still the most important issue to be addressed. Additionally, other critical problems such as thermal stability, photochemical stability, optical transparency, and processability in a given chromophore are followed. Moreover, polymer matrices with good thermal stability and appropriate polarity to host chromophores to meet effective poling and device processing conditions are still required.

There is already a wide choice of fluorescent molecular sensors for particular applications. However, there is still a need for sensors with improved selectivity and minimum perturbation to the microenvironment to be probed during the analysis. 
Moreover, the shortcoming of current used NIR probes was addressed by the development of simpler small molecular NIR probes with small HOMO-LUMO gaps for biological and biochemical analytes (e.g., amino acids) and in vivo use.

This research is particularly interested in development of dipolar zwitterionic chromophores for a variety of applications. Compared with the multi-step synthesis of the extensive conjugations for neutral chromophores, zwitterionic chromophores are typically easier to be prepared and have advantages in chemical and thermal stabilities. They are distinguished as solvent polarity indicators for their large negative solvatochromic effect. In addition, their large hyperpolarizabilities provide NLO properties for EO application, their relatively small sizes and large dipole moments are also of great importance for the efficient electric poling. Moreover, small band gap and NIR fluorescence properties make them potential NIR molecular fluorescence probes.

Zwitterionic chromophore PQDM was first successfully synthesized with high yield by our group, the EO activities were studied and low EO coefficient was obtained $\left(r_{33}=21 \mathrm{pm} / \mathrm{V}\right)$ in a guest-host polymer with low loading density. ${ }^{89}$ A higher $r_{33}$ of 45 $\mathrm{pm} / \mathrm{V}$ was obtained in a side-chain polyimide system ${ }^{56}$ and was improved to $65 \mathrm{pm} / \mathrm{V}$ in a hyperbranched PQDM doped polymer. ${ }^{90}$ In order to reduce the intermolecular aggregation, PeQDMs were developed later by introducing an ester group to the acceptor moiety. However, $r_{33}$ values are still much less than the theoretical prediction ${ }^{52}(210$ $\mathrm{pm} / \mathrm{V}$ ) and a tradeoff of number density and macroscopic nonlinearity were observed, indicating that the material still have strong aggregation. ${ }^{91}$

We have maintained an interest in this class of chromophore. To realize the full potential application of zwitterionic chromophores, it is required to better understand the 
structure-property relationship regarding the strength of donor, acceptor and conjugated length, two series of zwitterionic chromophores based on PQDM were designed and proposed as being ideal for achieving highly efficient poling.

To reduce the molecular aggregation and increase the EO coefficient, the first approach is "controlling the chromophore structure" through control of the size and shape of the chromophores, by introducing different groups from $\mathbf{R} \mathbf{1}$ to $\mathbf{R 4}$ to obtain increased molecular size and a spherical shape of the chromophores. The second approach is applying the "site-isolation principle", 92 to minimize undesirable dipole-dipole interactions in any polymer system that contains such compounds. Dicyanovinyl and 3CNQ were chosen as acceptors because particularly large molecular hyperpolarizabilities were found when they were present in reported NLO chromophores. Figure 1.14 shows the strategies to modify the PQDM chromophores.

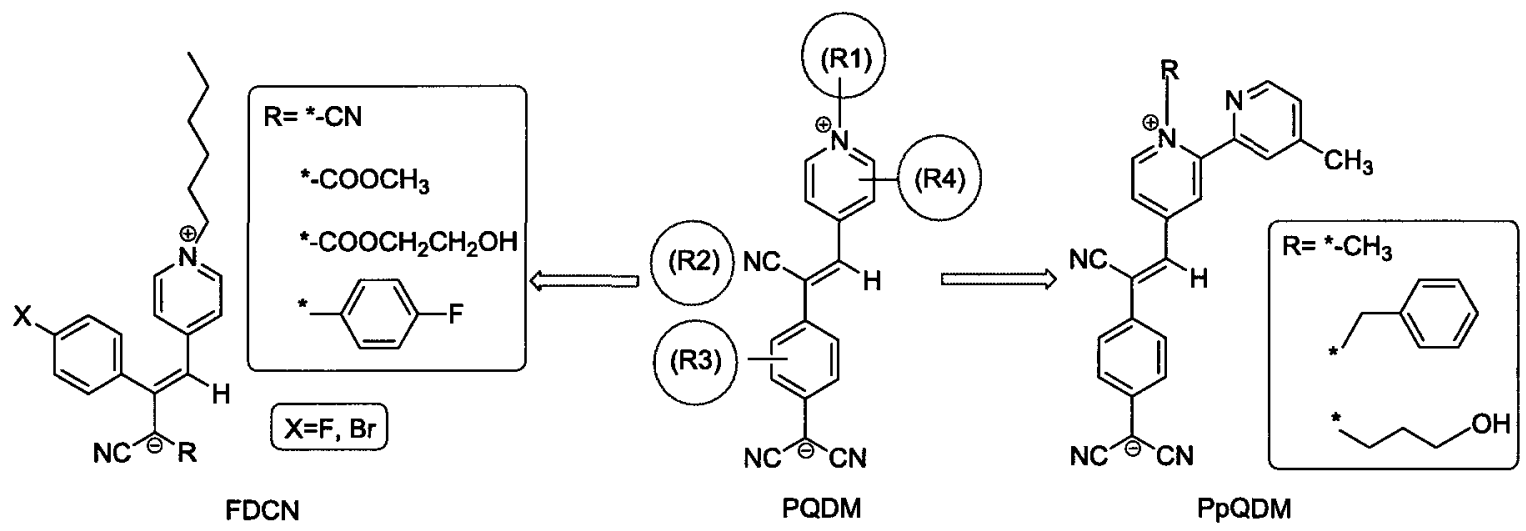

Figure 1.14. Potential modifications of PQDM and two new series of chromophores.

FDCN chromophores were designed based on the pyridinium donors, with a shorter conjugation to experimentally evaluate the dipole moment, and at the same time, lessen the structural complexity of the molecules. Aromatic bulk group was introduced to 
the central moiety to reduce the $\pi-\pi$ stacking. Halides on the aromatic rings were added (e.g., $\mathrm{F}, \mathrm{Br}$ ) to make the central part more bulky, and finally long aliphatic chains on the donor were used to help improve the solubility.

For chromophores PpQDM, dipyridylium was chosen as the donor to increase the donor size and reduce molecule symmetry and longer conjugation with stronger acceptor $3 \mathrm{CNQ}$ was expected to enlarge the $\mu \beta$ value. Careful selection of the hydroxyl alkyl halide is necessary to control the physical properties of the chromophore when grafted into a host polymer. For example, the length of the alkyl chain will determine the ability of the chromophore to orientate during poling since the alkyl chain represents the polymer chromophore spacer. These chromophores allow for functionalization at the donor side of the molecule for increasing solubility, for grafting, and/or for cross-linking, and improve the highly desirable NLO properties. All of the above features make these two series of chromophores very promising for NLO applications.

Another specific characteristic of FDCN and PpQDM chromophores is that the two cyano groups in dicyanovinyl and 3CNQ make them not only strong acceptors which contributes to the optical response, and also can function as ligands to coordinate to metal ions unlike the common optical chemosensors, which need communication between a binding site and a chromophore in a molecule. ${ }^{93}$ This allows zwitterionic dyes, which consists of a binding site in the chromophore to be promising molecular probe candidates to recognize specific guests through binding sites and produce a direct change in optical properties (absorption or fluorescence). PpQDM chromophores are expected to exhibit NIR fluorescent property due to their longer conjugation length. 
According to the above rationale, the main objectives of this research were as following:

1) In the first part of this work, we synthesized two new series of zwitterionic chromophores and their corresponding NLO chromophore-polymers.

2) In the next part, we systematically studied the relationship between the structure of these zwitterionic chromophores and their EO properties.

3) Finally, we investigated the NIR fluorescent property of the PpQDM chromophores, and then whether the PpQDM chromophores can form organometallic complexes to realize selective NIR biochemical sensing. 


\subsection{References}

(1) Bell, N. A.;Crouch, D. J.;Simmonds, D. J.; Goeta, A. E.; Gelbrich, T.; Hursthouse, M. B. J. Mater. Chem. 2002, 12, 1274.

(2) (a)Marder, S. R.; Gorman, C. B.; Meyers, F.; Perry, J. W.; Bourhill, G.; Bredas, J.-L.; Pierce, B. M. Science 1994, 265, 632. (b)Szablewski, M.; Thomas, P. R.; Thornton, A.; Bloor, D.; Cross, G. H.; Cole, J. M.; Howard, J. A. K.; Malagoni, M.; Meyers, F.; Bredas, J.-L.; Wenseleers, W.; Goovaerts, E. J. Am. Chem. Soc. 1997, 119, 3144. (c)Kang, H.; Facchetti, A.; Jiang, H.; Cariati, E.; Righetto, S.; Ugo, R.; Zuccaccia, C.; Macchioni, A.; Stern, C. L.; Liu, Z.; Ho, S.-T.; Brown, E.C.; Mark A. Ratner, M. A.; Marks, T. J. J. Am. Chem. Soc. 2007, 129, 3267.

(3) Higashino, K.; Nakaya, T.; Ishiguro, E. J. Photochem. Photobiol. A: Chem. 1994, 79,81 .

(4) Ashwell, G. J. Thin Solid Films 1990, 186, 155.

(5) (a)Metzger, R. M.; Chen, B.; Hopfner, U.; Lakshmikantham, M. V.; Vuillaume, D.; Kawai, T.; Wu, X.; Tachibana, H.; Hughes, T. V.; Sakurai, H.; Baldwin, J. W.; Hosch, C.; Cava, M. P.; Brehmer, L.; Ashwell, G. J. J. Am. Chem. Soc. 1997, 119 , 10455. (b)Metzger, R. M. Acc. Chem. Res. 1999, 32, 950.

(6) Neilands, O. Mol. Cryst. Liq. Cryst. 2001, 355, 331.

(7) (a)Andreu, R.; Garin, J.; Orduna, J.; Alcala, R.; Villacampa, B. Org. Lett. 2003, 5, 3143. (b)Kay, A. J.; Woolhouse, A. D.; Zhao, Y.; Clays, K.; J. Mater. Chem. 2004, 14, 1321. (c)Schmidt, J.; Schmidt, R.; Wurthner, F. J. Org. Chem. 2008, 73, 6355.

(8) Wolf, J. J.; Wortmann, R. Adv. Phys. Org. Chem. 1999, 32, 121.

(9) (a)Marder, S. R.; Perry, J. W.; Bourhill, G.; Gorman, C. B.; Tiemann, B. G.; Mansour, K. Science 1993, 261, 186. (b)Gorman, C. B.; Marder, S. R. Proc. Natl. Acad. Sci. USA 1993, 90, 11297.

(10) Oudar, J. L.; Chemla, D. S. J. Chem. Phys. 1977, 66, 2664.

(11) Reichardt, C. Chem. Rev. 1994, 94, 2319.

(12) (a) Nonlinear Optics of Organic Molecules and Polymers; Nalwa, H. S.; Miyata, S., Eds.; CRC Press: Boca Raton, 1997. (b)Burland, D. M.; Miller, R. D.; Walsh, 
C. A. Chem. Rev. 1994, 94, 31. (c)Kajzar, F.; Lee, K. S.; Jen, A. K-Y. Adv. Polym.

Sci. 2003, 161, 1. (d)Luo, J. D.; Zhou, X.; Jen, A. K.-Y. J. Mater. Chem. 2009, 19,7410 .

(13) (a)Lee, M.; Katz, H. E.; Erben, C.; Gill, D. M.; Gopalan, P.; Heber, J. D.; McGee, D. J. Science 2002, 298, 1401. (b)Liu, A. S.; Jones, R.; Liao, L.; Samara-Rubio, D.; Rubin, D.; Cohen, O.; Nicolaescu R.; Paniccia, M. Nature, 2004, 427, 615.

(14) Kaminov, I. P.; Turner, E. H. Proc. IEEE 1966, 54, 1374.

(15) (a)Ma, H.; Liu, S.; Luo, J. D.; Suresh, S.; Liu, L.; Kang, S. H.; Haller, M.; Sassa, T.; Dalton, L. R.; Jen, A. K.-Y. Adv. Funct. Mater. 2002, 12, 566. (b)Dalton, L. R.; Sullivan, P.; Bale, D. H. Chem. Rev. 2010, 110, 25.

(16) (a)Prasad, P. N.; Williams, D. J. Introduction to Nonlinear Optical Effects in Molecules and Polymers; John Wiley and Sons: New York, 1991. (b) Singer, K. D.; Kuzyk, M. G.; Sohn, J. E. J. Opt. Soc. Am. B 1987, 4, 968.

(17) (a)Bourhill, G.; Bredas, J. L.; Cheng, L.-T.; Marder, S. R.; Meyers, F.; Perry, J. W.; Tiemann, B. G. J. Am. Chem. Soc. 1994, 116, 2619. (b)Brown, E. C.; Marks, T. J.; Ratner, M. A. J. Phys. Chem. B 2008, 112, 44. (c)Di Bella, S.; Marks, T. J.; Ratner, M. A. J. Am. Chem. Soc. 1994, 116, 4440.

(18) Kuzyk, M. G.; Dirk, C. W. Characterization Techniques and Tabulations for Organic Nonlinear Optical Materials; Marcel Dekkar: New York, 1998.

(19) (a)Dalton, L. R.; Harper, A. W.; Robinson, B. H. Proc. Natl. Acad. Sci. U.S.A. 1997, 94, 4842. (b) Robinson, B. H.; Dalton, L. R. J. Phys. Chem. A 2000, 104, 4785.

(20) (a)Kanis, D. R.; Ratner, M. A.; Marks, T. J. Chem. Rev. 1994, 94, 195. (b) Oudar, J. L. J. Chem. Phys. 1977, 67, 446.

(21) (a)Cheng, L. T.; Tam, W.; Marder, S. R.; Stiegman, A. E.; Rikken, G.; Spangler, C. W. J. Phys. Chem. 1991, 95, 10643. (b)Cheng, L. T.; Tam, W.; Stevenson, S. H.; Meredith, G. R.; Rikken, G.; Marder, S. R. J. Phys. Chem. 1991, 95, 10631.

(22) Kippelen, B.; Peyghambarian, N.; Adv. Polym. Sci. 2003, 161, 87.

(23) Marder, S. R.; Kippelen, B.; Jen, A. K.-Y.; Peyghambarian, N. Nature, 1997, 388, 845. 
(24) Zhang, C.; Dalton, L. R.; Chem. Mater. 2001, 13, 3043.

(25) Kay, A. J.; Woolhouse, A. D.; A. D.; Zhao, Y.; Clays, K.; J. Mater. Chem. 2004, 14,1321 .

(26) Davydov, B. L.; Derkacheva, L. D.; Dunina, V. V.; Zhabotinskii, M. E.; Zolin, V. K.; Kreneva, L. C.; Samokhina, M. A. JEPT Lett. 1970, 12, 16.

(27) Rao, V. P.; Cai, Y. M.; Jen, A. K.-Y. J. Chem. Soc. Chem. Comm. 1994, 1689.

(28) (a)Staub, K.; Levina, G. A.; Barlow, S.; Kowalczyk, T. C.; Lackritz, H. S.; Barzoukas, M.; Fort, A.; Marder, S. R. J. Mater. Chem. 2003, 13, 825. (b) Twieg, R. J.; Burland, D. M.; Hedrick, J. L.; Lee, V. Y.; Miller, R. D.; Moylan, C. R.; Volksen, W.; Walsh, C. Mater. Res. Soc. Symp. Proc. 1994, 328, 421.

(29) Jen, A. K.-Y.; Cai, Y. M.; Bedworth, P. V.; Marder, S. R. Adv. Mater. 1997, 9, 132.

(30) Jen, A. K.-Y.; Rao, V. P.; Wong, K. Y.; Drost, K. J. J. Chem. Soc. Chem. Commun. 1993, 90.

(31) Dirk, C. W.; Katz, H. E.; Schilling, M. L.; King, L. A. Chem. Mater. 1990, $2,700$.

(32) Melikian, G.; Rouessac, F. P.; Alexandre, C. Synth. Commun. 1995, 25, 3045.

(33) (a)Dalton, L.; Harper, A.; Ren, A.; Wang, F.; Todorova, G.; Chen, J.; Zhang C.; Lee, M. Ind. Eng. Chem. Res., 1999, 38, 8. (b)Hua, J.; Luo, J. D.; Qin, J.; Shen, Y.; Zhang, Y.; Lu, Z. J. Mater. Chem. 2002, 12, 863.

(34) Robinson, B. H.; Dalton, L. R.; Harper, A. W.; Ren, A.; Wang, F.; Zhang, C.; Todorova, G.; Lee, M.; Aniszfeld, R.; Garner, S.; Chen, A.; Steier, W. H.; Houbrecht, S.; Persoons, A.; Ledoux, I.; Zyss, J.; Jen, A. K.-Y. Chem. Phys. 1999, 245,35 .

(35) Shi, Y.; Zhang, C.; Zhang, H.; Bechtel, J. H.; Dalton, L. R.; Robinson, B. H.; Steier, W. H. Science 2000, 288, 119.

(36) (a)Luo, J. D.; Haller, M.; Ma, H.; Liu, S.; Kim, T.-D.; Tian, Y.; Chen, B.; Jang, S.-H.; Dalton, L. R.; Jen, A. K.-Y. J. Phys. Chem. B 2004, 108, 8523. (b)Liao, Y.; Eichinger, B. E.; Firestone, K. A.; Haller, M.; Luo, J. D.; Kaminsky, W.; Benedict, J. B.; Reid, P. J.; Jen, A. K.-Y.; Dalton, L. R.; Robinson, B. H. J. Am. Chem. Soc. 2005, 127, 2758. (c)Kim, T.-D.; Kang, J.-W.; Luo, J. D.; Jang, S.-H.; Ka, J.-W.; 
Tucker, N.; Benedict, J. B.; Dalton, L. R.; Gray, T.; Overney, R. M.; Park, D. H.; Herman, W. N.; Jen, A. K.-Y. J. Am. Chem. Soc. 2007, 129,488.

(37) Cai, C.; Liakatas, I.; Wong, M.-S.; Busch, M.; Bosshard, C.; Gunter, P.; Concilio, S.; Tirelli, N.; Suter, U. W. Org. Lett. 1999, 1, 1847.

(38) Prasad, P.; Williams, D. Nonlinear Optical Effects in Molecules and Polymers; John Wiley and Sons: New York, 1991.

(39) (a)Cheng, L. T.; Tam, W.; Marder, S. R.; Steigman, A. E.; Rikken, G.; Spangler, C. W. J. Phys.Chem.1991, 95, 10643. (b)Ahlheim, M.; Barzoukas, M.; Bedworth, P. V.; Blanchard-Desce, M.; Fort, A.; Hu, Z.; Marder, S. R.; Perry, J. W.; Ruser, C.; Staehelin, M.; Zysset, B. Science 1996, 271, 335.

(40) (a)Albert, I. D. L.; Marks, T. J.; Ratner, M. A. J. Am. Chem. Soc. 1997, 119, 6575. (b)Rao, V. P.; Jen, A. K.-Y.; Chandrasekhar, J.; Namboothiri, I. N. N.; Rathna, A. J. Am. Chem. Soc. 1996, 118, 12443. (c)Raposo, M. M. M.; Sousa, A. M. R. C.; Kirsch, G.; Ferreira, F.; Belsley, M.; De Matos Gomes, E.; Fonseca, A. M. C. Tetrahedron 2005, 61, 11991.

(41) (a)Chen, D.; Fetterman, H. R.; Chen, A.; Steier, W. H.; Dalton, L. R.; Wang, W.; Shi, Y. Appl. Phys. Lett. 1997, 70, 3335. (b)Mingqian, H.; Leslie, T. M.; Sinicropi, J. A. Chem. Mater. 2002, 14, 4662.

(42) Zhang, C.; Ren, A. S.; Wang, F.; Zhu, J.; Dalton, L. R. Chem. Mater. 1999, 11, 1966.

(43) Shu, C. F.; Shu, Y. C.; Gong, Z. H.; Peng, S. M.; Lee, G. H.; Jen, A. K.-Y. Chem. Mater. 1998, 10, 3284.

(44) Metzger, R. M.; Heimer, N. E.; Aswell, G. J. Mol. Cryst. Liq. Cryst. 1984, 107, 133. (b)Bell, N. A.; Crouch, D. J.; Simmonds, D. J.; Goeta, A. E.; Gelbrich, T.; Hursthouse, M. B. J. Mater. Chem. 2002, 12, 1274.

(45) (a)Broo, A.; Zerner, M. C. Chem. Phys. 1995, 196, 407. (b)Broo, A.; Zerner, M. C. Chem. Phys. 1995, 196, 423.

(46) (a)Dulcic, A.; Flytzanis, C. Opt. Commun. 1978, 25, 402. (b)Benson, H. G.; Murrell, J. N. J. Chem. Soc., Faraday Trans. 2 1972, 68, 137. 
(47) Kay, A. J.; Woolhouse, A. D.; Gainsford, G. J.; Haskell, T. G.; Barnes, T. H. J. Mater. Chem. 2001, 11, 996.

(48) Abbotto, A.; Beverina, L.; Bradamante, S.; Facchetti, A.; Klein, C.; Pagani, G. A.; Redi- Abshiro, M.; Wortmann, R. Chem. Eur. J. 2003, 9, 1991.

(49) Ashwell, G. J. Organic Materials for Nonlinear Optics; Ashwell, G. J.; Bloor, D. Eds; Royal Soc. Of Chem.: Cambridge, 1993.

(50) (a)Acker, D. S.; Harder, R. J.; Hertler, W. R.; Mahler, W.; Melby, L. R.; Benson, R. E. ; Mochel, W. E. J. Am. Chem. Soc. 1960, 82, 6408. (b)Acker, D. S.; Hertler W. R. J. Am. Chem. Soc. 1962, 84, 3370.

(51) Akhtar, S.; Tanaka, J.; Metzger R. M.; Ashwell, G. J. Mol. Cryst. Liq. Cryst. 1986, $139,353$.

(52) Cross, G. H.; Hackman, N. A.; Thomas, P. R.; Szablewski, M.; Palsson, L. O.; Bloor, D. Opt. Mater. 2002, 21, 29.

(53) Burland, D. M.; Miller. R. D.; Walsh, C. A. Chem. Rev. 1994, 94, 31.

(54) (a)Shuto, Y.; Anmno, M.; Kaino, T. Jpn. J. Appl. Phys. 1991, 30, 320. (b)Chen, D.; Fetterman, H.; Chen, A.; Sterier, W.; Dalton, L. Appl. Phys. Lett. 1997, 77, 3335 .

(55) Jen, A. K.-Y.; Wong, K. Y.; Rao, V. P.; Drost, K. J. Electron. Mater. 1994, 23, 653.

(56) Cai, Y. M.; Jen, A. K.-Y. Appl. Phys. Lett. 1995, 117, 7295.

(57) Xu, C.; Wu, B.; Becker, M. W.; Dalton, L. R.; Ranon, P. M.; Shi, Y.; Steier, W. H. Chem. Mater. 1993, 5, 1439.

(58) Moon, K.-J.; Shim, H.-K.; Lee, K.-S.; Zieba, J.; Prasad, P. N. Macromolecules 1996, 29, 861 .

(59) Yoon, C. B.; Shim, H. K. J. Mater. Chem. 1999, 9, 2339.

(60) Song, N. H.; Men, L.; Gao, J. P.; Bai, Y. W.; Beaudin, A. M. R.; Yu, G. M.; Wang, Z. Y. Chem. Mater. 2004, 16, 3708.

(61) (a)Zhu, P.; Wang, P.; Wu, W.; Ye, C. J. Non. Opt. Phys. Mater. 1999, 8, 461. (b) Qin, A.; Yang, Z.; Bai, F.; Ye, C. J. Polym. Sci.: Part A: Polym. Chem. 2003, 41, 2846. 
(62) Luo, J. D.; Haller, M.; Li, H.; Tang, H. Z.; Jen, A. K.-Y.; Jakka, K.; Chou, C. H.; Shu, C. F. Macromolecules 2004, 37, 248.

(63) Luo, J. D.; Haller, M.; Li, H.; Kim, T.-D.; Jen, A. K.-Y. Adv. Mater. 2003, 15, 1635.

(64) Anslyn, E. V. J. Org. Chem. 2007, 72, 687.

(65) Beer, P. D.; Cadman, J. Coord. Chem. Rev. 2000, 205, 131.

(66) Bissel, R. A; De Silva, A. P.; Gunaratne, H. Q. N.; Lynch, P. L. M.; Maguire, G. E. M.; Sandanayake, K. R. A. S. Chem. Soc. Rev. 1992, 187.

(67) De Silva, A. P.; Nimal Gunaratne, H. O.; Gunnlaugusson, T.; Huxley, A. J. M.; McCoy, C. P.; Rademacher, J. T.; Rice, T. E. Chem. Rev. 1997, 97, 1515.

(68) Nguyen, B. T.; Anslyn, E. V. Coord. Chem. Rev. 2006, 250, 3118.

(69) Ramachandaram, B.; Samanta, A. Chem. Commun. 1997, 1037.

(70) Mitchell, K.A.; Brown, R. G.; Yuan, D.; Chang, S.-C.; Utecht, R. E.; Lewis, D. E. J. Photochem. Photobiol. A: Chem. 1998, 115,157.

(71) Fabbrizzi, L.; Licchelli, M.; Pallavicini, P.; Perotti, A.; Taglietti, A.; Sacchi, D. Chem. Eur. J. 1996, 2, 75.

(72) Fabbrizzi, L.; Licchelli, M.; Pallavicini, P.; Perotti, A.; Sacchi, D. Angew. Chem. Int. Ed. Engl. 1994, 33, 1975.

(73) Sasaki, D. Y.; Shnek, D. R.; Pack, D. W.; Arnold, F. H. Angew. Chem. Int. Ed. Engl. 1995, 34, 905.

(74) Ohler, J. Y. N. E.; Vance, D. H.; Aumiller, W. D.; Czarnik, A. W. Tetrahedron Lett. 1997, 38, 3845.

(75) Parker, D.; Williams, J. A. G. J. Chem. Soc. Perkin Trans. 2 1995, 1305.

(76) Beeby, A.; Parker, D.; Williams, J. A. G. J. Chem. Soc. Perkin Trans. 2 1996, 1565.

(77) De Santis, G.; Fabbrizzi, L.; Licchelli, M.; Mangano, C.; Sacchi, D.; Sardone, N. Inorg. Chim. Acta 1997, 257, 69.

(78) Deviprasad, G. R.; D'Souza, F. Chem. Comm. 2000, 1915.

(79) Hilderbrand, S. A.; Lim, M. H.; Lippard, S. J. J. Am. Chem. Soc. 2004, 126, 4972. 
(80) Hortala, M. A.; Fabbrizzi, L.; Marcotte, N.; Stomeo, F.; Taglietti, A. J. Am. Chem. Soc. 2003, 125, 20-21.

(81) Richards-Kortum, R.; Sevick-Muraca, E. Annu. Rev. Phys. Chem., 1996, 47, 555.

(82) (a)Patonay, G.; Antoine, M. D. Anal.Chem.1991, 63, 321. (b)Stoyanov, S. Pract. Spectrosc. 2001, 25, 35.

(83) (a)Achilefu, S. Technol. Cancer Res. Treat. 2004, 3, 393. (b)Hilderbrand, S. A.; Weissleder, R. Curr. Opin. Chem. Biol. 2010, 14, 71.

(84) Achilefu, S. Angew. Chem. Int. Ed. 2010, 49, 9816.

(85) (a)Perepichka, D. F.; Bryce, M. R. Angew. Chem. Int. Ed. 2005, 44, 5370. (b) Roncali, J. Chem. Rev. 1997, 97, 173.

(86) Smith, G. J.; Dunford, C. L.; Kay, A. J.; Woolhouse, A. D. J. Photochem. Photobiol. A: Chem. 2006, 179, 237.

(87) Wu, Y. L; Bures, F; Jarowski, P. D.; Schweizer, W. B.; Boudon, C.; Gisselbrecht, J-P; Diederich, F. Chem. Eur. J. $2010,16,9592$.

(88) Ying Xiong "Development of Zwitterinic Chromophores for Electro-Optic Applications", Ph.D. Thesis, Carleton University, 2008.

(89) Beaudin, A. M. R.; Song, N. H.; Bai, Y. W.; Men, L.; Gao, J. P.; Wang, Z. Y.; Szablewski, M; Cross, G.; Wenseleers, W. Campo, J.; Goovaerts, E. Chem. Mater. 2006, 18, 1079 .

(90) Bai, Y. W.; Song, N. H.; Gao, J. P.; Sun, X; Wang, X. M.; Yu, G. M.; Wang, Z. Y. J. Am. Chem. Soc. 2005, 127, 2060.

(91) Xiong, Y.; Tang, H.; Zhang, J.; Wang, Z.Y.; Campo, J.; Wenseleers, W.; Goovaerts, E. Chem. Mater. 2008, 20, 7465.

(92) Luo, J. D.; Liu, S.; Haller, M.; Liu, L.; Ma, H.; Jen, A. K.-Y. Adv. Mater. 2002, $14,1763$.

(93) Czarnik, A. W. Acc. Chem. Res. 1994, 27, 302. 


\section{Chapter 2 Synthesis and Characterization of Zwitterionic Chromophores}

\section{$2.1 \quad$ Introduction}

According to the BLA model, ${ }^{1}$ LHS chromophores with neutral ground states have positive $\beta$ values; while RHS zwitterionic chromophores with charge-separated ground states have negative $\beta .^{2}$ Although the absolute value of $\beta$ is the only consideration for NLO chromophores for EO application, predominant efforts focus on the exploration of neutral chromophores and less attention has been paid to zwitterionic chromophores despite the considerable advantages displayed by zwitterionic chromophores including 1) ease of synthesis, 2) good thermal stability, 3) high ground state dipole moment and 4) larger nonlinearity than the neutral push-pull system of similar conjugation length. ${ }^{3}$

Among the many types of zwitterions, derivatives of TCNQ such as $\beta$-[4pyridinium]- $\alpha$-cyano-4-styryldicyano-methanide, (trivial name: pyridinium tricyanoquinonedimethane, PQDM) (Scheme 2.1), which was first reported by Metzger and Ashwell ${ }^{4}$ and calculated to have a large static hyperpolarizability of $1270 \times 10^{-30}$ esu. ${ }^{5}$ The stabilization gained in the charge-separated state due to the increase in aromaticity of the negatively charged 3CNQ acceptor represents a huge driving force for these chromophores used in EO applications. This stabilization has been confirmed by Xray crystallography, infrared (IR), and nuclear magnetic resonance (NMR) spectroscopy. ${ }^{6}$ However, these highly crystalline chromophores have no functional groups, which makes it very difficult to chemically incorporate them into a polymer host.

Our group is particularly interested in functionalization of PQDM due to the desirable figures of merits, 1) large $\beta$ values, comparable to some well-studied neutral ground state chromophores, such as FTC $\left.\left(\mu \beta=18,000 \times 10^{-48} \mathrm{esu}\right) ; 2\right)$ large dipole 
moment $(43 \pm 8 \mathrm{D}) ;{ }^{7}$ ) relatively small size, which can be easily prepared via short synthetic routes; 4) good solubility in common organic solvents and good thermal stability (e.g. $>250{ }^{\circ} \mathrm{C}$ ). These advantages make PQDM the most promising candidate of zwitterionic chromophores for NLO applications.

The first reported synthesis of PQDM involved the condensation reaction between picolinium halide and lithium TCNQ adduct or TCNQ with low reaction yields (e.g., 6 $30 \%$ ) and long reaction time (5-14 days). ${ }^{8}$ In 2003 , our group reported a drastic improvement in reaction time and yield (97\%) for the PQDM synthesized from two equivalents of lithium TCNQ adduct with one equivalent of picolinium halide in refluxing acetonitrile with the presence of two equivalents of a strong organic base $(1,8$ diazabicyclo[5.4.0] undec-7-ene, DBU) (Scheme 2.1). ${ }^{9}$<smiles>N#CC(C#N)=c1ccc(=C(C#N)C#N)cc1</smiles>

TCNQ<smiles>N#CC(=N)c1ccc(C(=[W])C#N)cc1</smiles>

TCNQLI

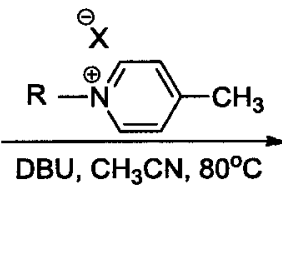<smiles>C=C(C)[C+](C#N)C=N</smiles>

PQDM

Scheme 2.1. Synthesis of PQDM.

Following the successful synthesis of PQDM, a series of hydroxyl-containing PQDM derivatives with different $\mathrm{R}$ groups, such as hydroxypropyl and hydroxyhexyl were synthesized by our group in 2006 (e.g., PQDM-C3OH in Chart 2.1). These chromophores were successfully covalently bonded to the polyimide as side chains. However, the functionalization of PQDM has mainly focused on introducing different substituents on the pyridinium ring (donor part), ${ }^{10}$ and no significant contribution on 
increasing the $\beta$ values was found in the modified PQDM. The poling efficiency and EO coefficient values of these polymer systems were still much lower than the theoretical prediction. The limitation of the above EO device is generally considered to be due to the strong intermolecular dipole-dipole interactions since dipolar chromophores are closely packed, poling-induced non-centrosymmetric alignment of these chromophores is very limited. ${ }^{11}$

In 2008 we developed a new series of functionalized zwitterionic picolinium dicyano-esterquinodimethane (PeQDM) chromophores (e.g., PeQDM-Ben in Chart 2.1), aiming to reduce the chromophore-chromophore interaction by introducing an ester group into the acceptor part. The high molecular hyperpolarizability was unexpectedly maintained, or even increased in comparison to the PQDM chromophores, despite the fact that the ester group is considered to be a weaker electron acceptor than the cyano group in PQDM. ${ }^{12}$

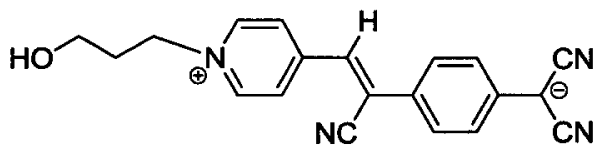

PQDM-C3OH

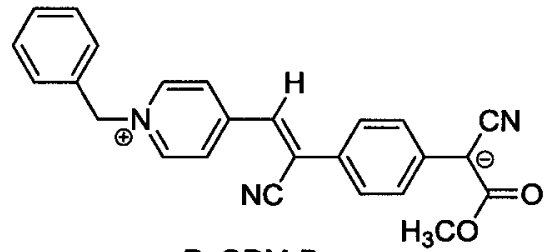

PeQDM-Ben

Chart 2.1. Chemical structures of PQDM-C3OH and PeQDM-Ben.

In order to understand the structure-property relationship of zwitterionic chromophores and realize the full potential of PQDM chromophores, this chapter describes the synthesis and structural characterization of two series of functionalized FDCN (Scheme 2.2) and PpQDM chromophores (Scheme 2.6) based on the design rationale described in Chapter 1 , as well as the X-ray crystallographic analysis, 
thermostability, negative solvatochromism, acid sensitivity, and microscopic NLO properties of these two series of chromophores.

\subsection{Results and Discussion}

\subsubsection{Synthesis and Characterizations of FDCN Chromophores}

\subsubsection{Synthesis of FDCN Chromophores}

Using 4-fluorophenylacetonitrile (or 4-bromophenylacetonitrile) and 4-pyridinecarboxaldehyde as starting materials, the synthesis of FDCN chromophores is depicted in Scheme 2.2. The pyridinium moiety with 6-carbon aliphatic chain in FDCN is considered to be a donor, whereas the acceptor part contains a cyano group and is chosen as the site of structural modification.

The crucial pyridinium salt donor intermediate 2 was prepared by alkylation of intermediate 1 and then the central cyano group of 2 was substituted with different acceptors. The target chromophores including a bromo-cyanide containing chromophore (BrDCN) and four fluoro-cyanide containing chromophores, with different aliphatic and aromatic groups as the acceptor, were prepared with high yield (60\%). Among the FDCN chromophores, hydroxyl-containing chromophore ( $\mathrm{FCNOH})$ is considered to be the potential monomer for making side-chain NLO polymers (UV spectra of FDCNOH in DMF and chloroform in Appendix B, Figure S2.7). The general procedure for the synthesis of 2-hydroxyethyl cyanoacetate ${ }^{13}$ was undertaken by esterification of cyanoacetic acid with 2-hydroxyethyl alcohol in benzene in the presence of $\mathrm{TsOH}$ as a catalyst; the molar ratio of cyanoacetic acid to the alcohol was 1:1 to 1:1.05 (Scheme 2.3). 


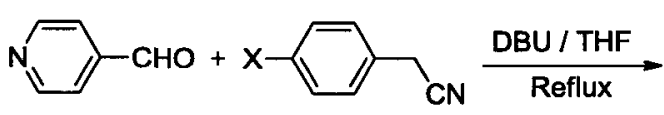

$$
\mathrm{X}=\mathrm{F}, \mathrm{Br}
$$<smiles>[X]c1ccc(/C(C#N)=C/c2ccncc2)cc1</smiles><smiles>CCCCCC(C)Br</smiles><smiles>[X]c1ccc(/C=C\C(C)C)cc1</smiles>

Scheme 2.2. Synthesis of crucial intermediate 2 and FDCN chromophores.

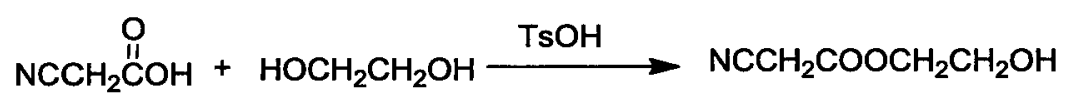

Scheme 2.3. Synthesis of 2-hydroxyethyl cyanoacetate.

\subsubsection{Characterizations of FDCN Chromophores}

All of the FDCN chromophores were fully characterized by ${ }^{1} \mathrm{H} N \mathrm{NR},{ }^{13} \mathrm{C} \mathrm{NMR}$, IR and mass spectrometry.

Figure 2.1 shows the ${ }^{1} \mathrm{H}$ NMR spectrum of FDCN with assigned peaks. In the spectrum, proton resonances (ppm) at $7.81(2 \mathrm{H}, \mathrm{d}, \mathrm{J}=7.1 \mathrm{~Hz}), 7.36(2 \mathrm{H}, \mathrm{d}, \mathrm{J}=8.7 \mathrm{~Hz})$, $7.32(2 \mathrm{H}, \mathrm{d}, \mathrm{J}=8.7 \mathrm{~Hz}), 6.34(2 \mathrm{H}, \mathrm{d}, \mathrm{J}=7.1 \mathrm{~Hz}), 5.88(1 \mathrm{H}, \mathrm{s}), 3.92(2 \mathrm{H}, \mathrm{t}), 1.22(6 \mathrm{H}, \mathrm{m})$ and $0.83(3 \mathrm{H}, \mathrm{t})$ were observed and are consistent with the zwitterionic structure of FDCN. 


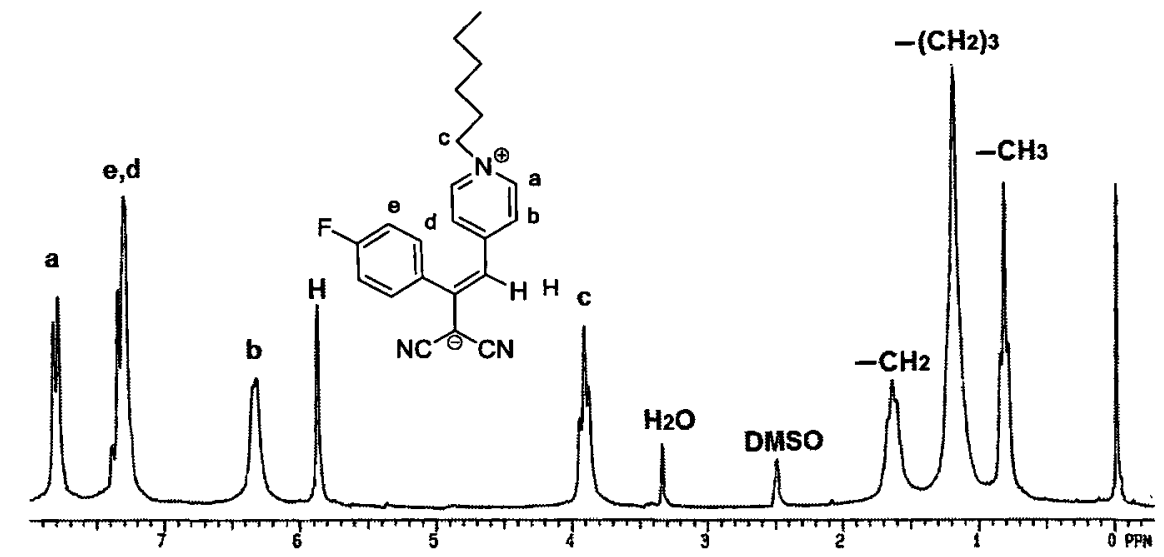

Figure 2.1. ${ }^{1} \mathrm{H}$ NMR spectrum $(300 \mathrm{MHz})$ of FDCN in DMSO- $d_{6}$.

Figure 2.2 displays the IR spectrum of FDCN. The cyano group is observed at $2192 \mathrm{~cm}^{-1}$, and the intensity of the peak is strikingly high, which is characteristic of the cyano group connected to an electron-rich group with a negative charge. The IR data accurately supports the zwitterionic structure of FDCN. Additionally, in the MS spectrum of FDCN (Appendix B, Figure S2.3), the molecular ion peak at m/z 347.1793, 348.1776 $[\mathrm{M}+\mathrm{H}]^{+}$and $370.1608[\mathrm{M}+\mathrm{Na}]^{+}$were observed and further support the structural characterization.

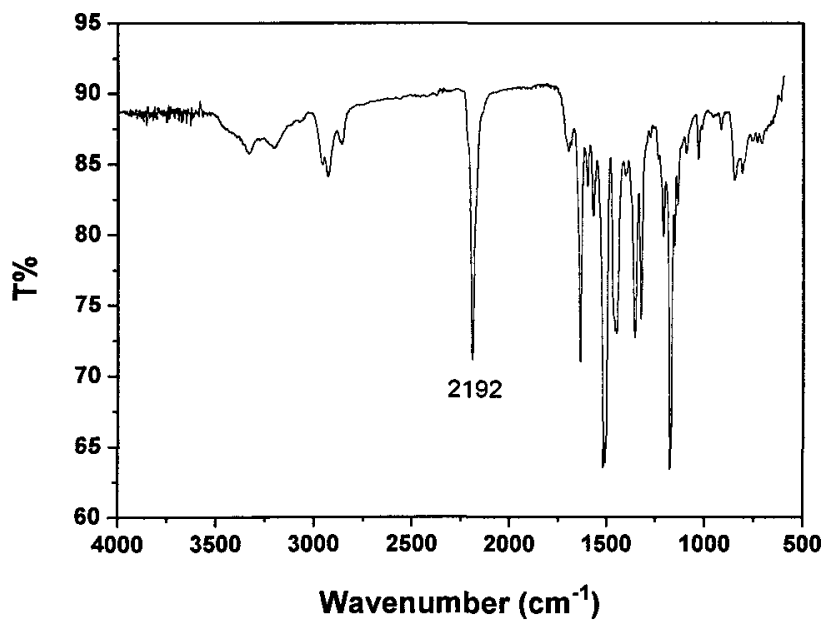

Figure 2.2. IR spectrum of FDCN ( $\mathrm{KBr}$ pellet). 
In order to illustrate the extent of ground-state charge separation, selected IR data of 2, FDCN, FCNE and other model compounds are listed in Table 2.1. Since the stretching frequency of the cyano groups is sensitive to the vicinal electron density, it is a good probe for the ground-state charge transfer. The bond strength and therefore the vibrational frequency of $\mathrm{CN}$ groups are reduced by the presence of increased vicinal electron density, which interacts with the $\pi^{*}$ orbital of the CN group. ${ }^{14}$

Table 2.1. Selected IR data for 2, FDCN, FCNE

\begin{tabular}{ll}
\hline Compound & $v_{\mathrm{CN}}\left(\mathrm{cm}^{-1}\right)$ \\
\hline $\mathrm{Li}^{+}\left[\mathrm{C}(\mathrm{CN})_{2} \mathrm{Ph}\right]^{-}$ & $2178^{17 \mathrm{a}}$ \\
TCNQ & $2226^{17 \mathrm{~b}}$ \\
$\mathbf{2}$ & $2224^{-19}$ \\
FDCN & 2192 \\
FCNE & 2180 \\
PQDM & $2173^{12}$ \\
PeQDM & $2146^{14}$ \\
\hline
\end{tabular}

For reference, the stretching frequency, $v_{\mathrm{CN}}=2178 \mathrm{~cm}^{-1}$, for lithium dicyanophenylmethanide $\left(\mathrm{Li}^{+}\left[\mathrm{C}(\mathrm{CN})_{2} \mathrm{Ph}\right]^{-}\right)$models a full formal vicinal negative charge; while the stretching frequency, $v_{\mathrm{CN}}=2226 \mathrm{~cm}^{-1}$, for TCNQ serves as a model without formal vicinal charge. ${ }^{15}$ The $\mathrm{CN}$ stretches at $2220 \mathrm{~cm}^{-1}$ and $2224 \mathrm{~cm}^{-1}$ for intermediate compounds $\mathbf{1}$ and $\mathbf{2}$, respectively, point to a small formal charge at the corresponding dicyanovinyl sites. On the other hand, the lower values for FDCN $\left(v_{\mathrm{CN}}=2192 \mathrm{~cm}^{-1}\right)$ and FCNE $\left(v_{\mathrm{CN}}=2180 \mathrm{~cm}^{-1}\right)$ imply considerable CT character in the ground state. The smaller $v_{\mathrm{C} \equiv \mathrm{N}}$ frequency for $\mathrm{FCNE}$ indicates that more aromatization was gained from ICT than FDCN. Similar comparison of the smaller $v_{\mathrm{C} \equiv \mathrm{N}}$ frequency of PeQDM $\left(2146 \mathrm{~cm}^{-1}\right)$ with that of PQDM $\left(2173 \mathrm{~cm}^{-1}\right)$ indicate that PeQDM with one cyano and one ester group 
at the acceptor part gain more aromatization stabilization than PQDM, which shows that PeQDM has a higher molecular hyperpolarizability and larger EO coefficient than PQDM.

\subsubsection{Structure Characterization by X-ray Crystallograph}

Further evidence for the formation of zwitterionic chromophores is provided by X-ray crystallography. Although it is often difficult to grow single crystals of highly dipolar dyes, crystal of FDCN suitable for crystallographic structure analysis and can be obtained by slow crystallization from a hexane/acetone solution at room temperature. Important crystallographic data for FDCN are collected in Appendix A. The molecular structure of FDCN and selected bond lengths of the crystal are shown in Figure 2.3 and Table 2.2 , respectively.

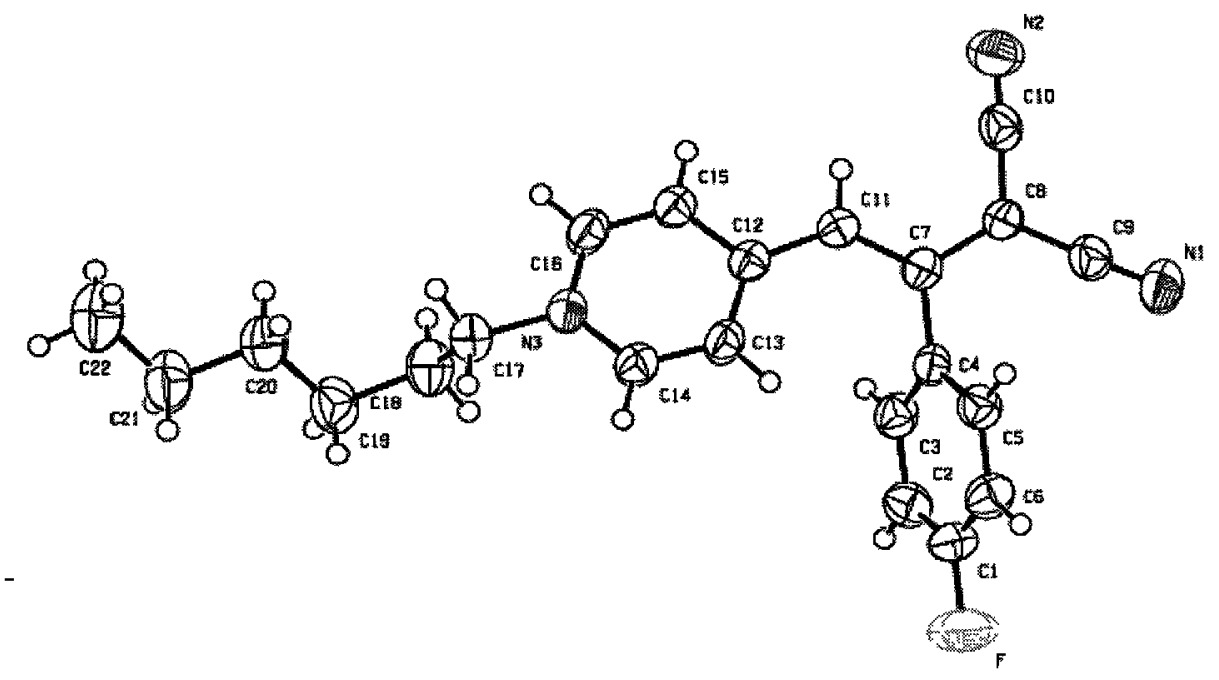

Figure 2.3. ORTEP drawing of the structure of FDCN in the crystal with atomic number. Hydrogen atoms were omitted for clarity.

Despite of the considerable number of bulky substituents, an almost undisturbed $\pi$-conjugated system, which is nearly coplanar, is observed and reveals significant bond 
length equilibration within the conjugated path. The central C7-C11 bond has a bond length of $1.384 \AA$, very similar to the length of a $\mathrm{C}=\mathrm{C}$ double bond is maintained (standard C-C single bond and double bond values are $1.46 \AA$ and $1.34 \AA$, respectively. ${ }^{16}$ ), indicating a significant displacement of electron density (or charge transfer) from the donor to the acceptor moieties, corresponding to a large contribution of the zwitterionic resonance structure in FDCN. This conclusion is also supported by additional bond lengths shown in Table 2.2 .

However, single bond character is observed between C7 and C8 (1.408 $\AA$ ) also evident from the observed C-CN bond (1.411 $\AA$ and $1.420 \AA$ in FDCN vs $1.427 \AA$ in typical TCNQs), indicating substantial negative charge localization within the $-\mathrm{C}(\mathrm{CN})_{2}$ group, and elongated CN $(1.151 \AA$ and $1.147 \AA)$ bond compared to that in typical TCNQs $(1.144 \AA)^{17}$ indicating a result of charge resonant stabilization via the two CN groups.

Table 2.2. Selected bond distances for FDCN in the crystal ${ }^{\mathrm{a}}$

\begin{tabular}{|c|c|c|c|c|c|}
\hline Atom1 & Atom 2 & $\bar{\AA}$ & Atom1 & Atom 2 & $\AA$ \\
\hline$\overline{\mathrm{C} 4}$ & $\mathrm{C7}$ & $1.487(2)$ & $\mathrm{C} 16$ & N3 & $1.352(1)$ \\
\hline $\mathrm{C} 7$ & $\mathrm{C} 11$ & $1.384(4)$ & $\mathrm{C} 14$ & N3 & $1.349(2)$ \\
\hline $\mathrm{C} 7$ & $\mathrm{C} 8$ & $1.408(2)$ & $\mathrm{C} 17$ & N3 & $1.480(1)$ \\
\hline $\mathrm{C} 11$ & $\mathrm{C} 12$ & $1.416(2)$ & $\mathrm{C} 8$ & $\mathrm{C} 9$ & $1.411(2)$ \\
\hline $\mathrm{C} 12$ & $\mathrm{C} 13$ & $1.409(2)$ & $\mathrm{C} 8$ & $\mathrm{C} 10$ & $1.420(2)$ \\
\hline $\mathrm{C} 12$ & $\mathrm{C} 15$ & $1.413(2)$ & $\mathrm{C} 9$ & N1 & $1.147(2)$ \\
\hline $\mathrm{C} 13$ & $\mathrm{C} 14$ & $1.352(2)$ & $\mathrm{C} 10$ & $\mathrm{~N} 2$ & $1.151(2)$ \\
\hline $\mathrm{C} 15$ & $\mathrm{C} 16$ & $1.349(2)$ & $\mathrm{C} 1$ & $\mathrm{~F}$ & $1.362(4)$ \\
\hline
\end{tabular}

${ }^{a}$ Esd's in parentheses refer to the last digit.

For comparison, the well-known Brooker dye 4-\{2-[1-methyl-4-(1H) pyridylidene] ethylidene cyclohexa-2,5-dien-1-one (Scheme 2.4), which has a similar conjugated backbone and a length of the central bond of $1.35 \AA$ in the solid state, is considered as the 
prototype of a zwitterionic merocyanine dye, with an estimated degree of $81.8 \%$ for the zwitterionic form. ${ }^{18}$ The longer central bond, $1.384 \AA$, in FDCN indicated that less than $80 \%$ of the zwitterionic form in its ground state. Compared to the central bond length of PeQDM (1.354 $\AA$, double bond character), FDCN has a lower degree of charge transfer in ground state than that of PeQDM.

Although the bond lengths in the conjugated bridge and acceptor part clearly demonstrated a zwitterionic molecular structure of FDCN, the bond length of the pyridinium ring is quinoidal rather than aromatic. Similar phenomena have also been reported for other zwitterionic molecules such as DEMI ${ }^{19}$ and PeQDM. ${ }^{12}$ Therefore, the best description of the ground state structure of such chromophores is the combination of the two limiting forms (Scheme 2.4), zwitterionic, and neutral forms with predominantly zwitterionic structure. The zwitterionic character is also verified by the negative solvatochromism of FDCN (Table 2.4 in Section 2.2.4).

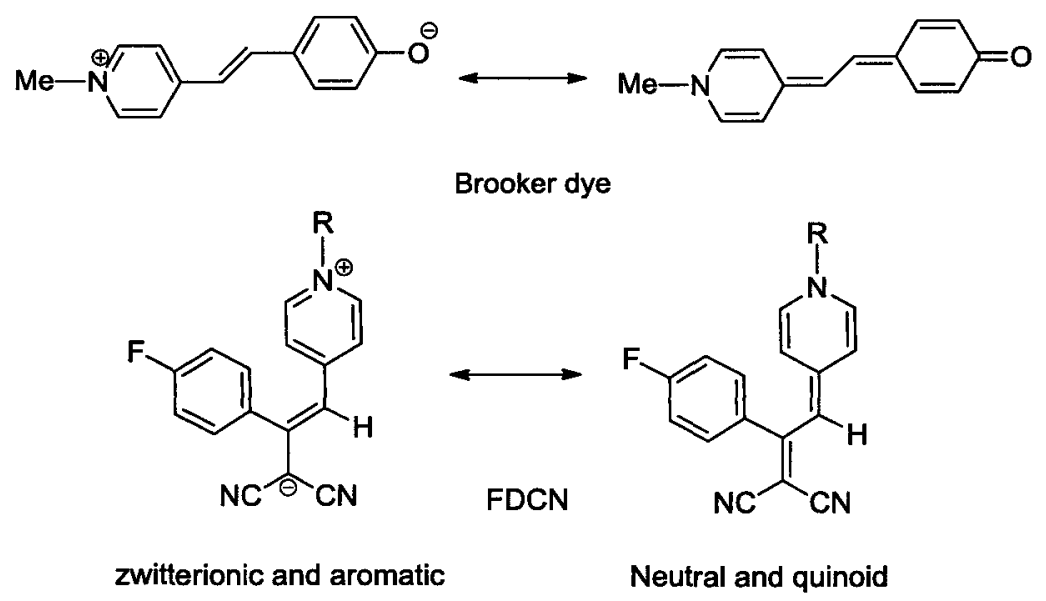

Scheme 2.4. Resonance structures of Brooker dye and FDCN.

The solid-state packing diagram of FDCN is shown in Figure 2.4 (left). Even in the presence of central bulky fluorophenyl substituents, a centrosymmetric unit of two 
FDCN is observed in the crystal cell, arrayed in an antiparallel fashion with an intermolecular distance of $4.0645 \AA$ between the aromatic backbones. Antiparallel overlap with respect to each other and a smaller slip angle $\left(\theta=48.8^{\circ}\right)$ than that in PeQDM-Ben $\left(\theta=64.5^{\circ}\right)$ is observed which indicated that there is no $\mathrm{H}$-aggregation between two chromophores. The intermolecular distance (d) is considerably larger than the sums of van der Waals radii between planar cofacial $\pi$-electron systems $(\sim 3.50 \AA)^{20}$ and result in the difficulty in close face-to-face packing of the aromatic rings in these central bulky molecules. Therefore, incorporation of bulk groups onto the central moiety is an effective strategy to minimize molecular interaction. The weak electrostatic interactions between FDCN (compared to PQDM and PeQDM) make this kind chromophore soluble not only in polar solvents such as DMF, acetonitrile (ACN) and methanol, but also moderately soluble in less polar solvents such as chloroform, tetrahydrofuran (THF) and benzene.

However, $\pi-\pi$ stacking formed between the substituted fluorophenyl rings (distance of $3.5 \AA$ between these two $\pi$ systems) is observed. Further increase the size of the central part of zwitterionic chromophore with bulky substituted group may effectively decrease the intermolecular distance.
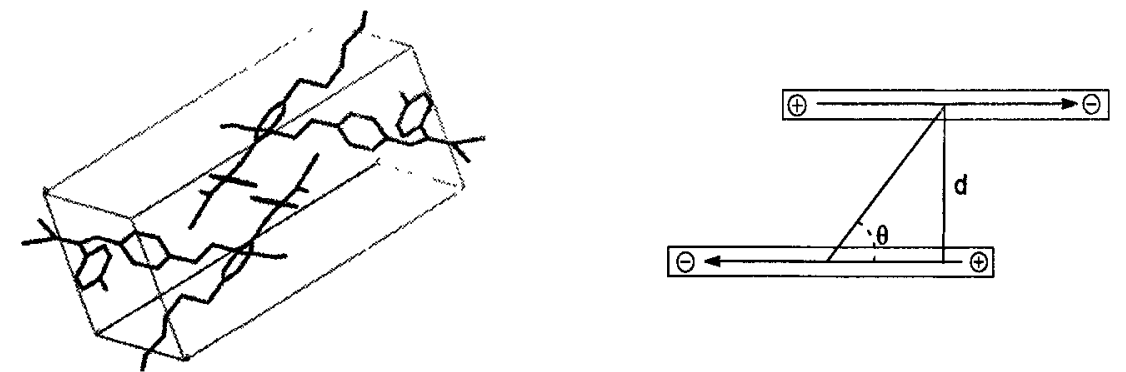

Figure 2.4. Crystal packing diagrams of chromophore FDCN (the hydrogen atoms are omitted for clarity (left) ; Model for a centrosymmetric dimer of two dipolar dyes, $\theta$ is the slip angle of the dimer, $d$ is the distance between the $\pi-\pi$ systems (right). 


\subsubsection{Thermal Analysis}

For EO application, thermal stability of the chromophore is a key parameter for the selection of host polymer and poling temperature. The thermal properties of FDCN and $\mathrm{BrDCN}$ were measured by thermogravimetric analysis (TGA) under nitrogen and onset temperatures for $5 \%$ weight loss were found at $312{ }^{\circ} \mathrm{C}$ and $323{ }^{\circ} \mathrm{C}$, respectively (Figure 2.5 left), such a thermal stability is higher than that of PQDM (Decomposition temperature, $\left.\mathrm{T}_{\mathrm{d}}=287^{\circ} \mathrm{C}\right)^{10}$ and PeQDMs $\left(215^{\circ} \mathrm{C}-287^{\circ} \mathrm{C}\right) .{ }^{12}$

Differential scanning calorimetry (DSC) studies of FDCN and BrDCN showed typical phase transition (Figure 2.5 right). Crystallization occurs at $130^{\circ} \mathrm{C}$ and $120^{\circ} \mathrm{C}$ respectively before the chromophores melt at $167^{\circ} \mathrm{C}$ and $196^{\circ} \mathrm{C}$. Cooling with rates of $10^{\circ} \mathrm{C} / \mathrm{min}$ does not lead to crystallization of these chromophores but to formation of amorphous solids. The propensity for glass formation was utilized on organic photorefractive materials based on merocyanine dyes. $^{21}$
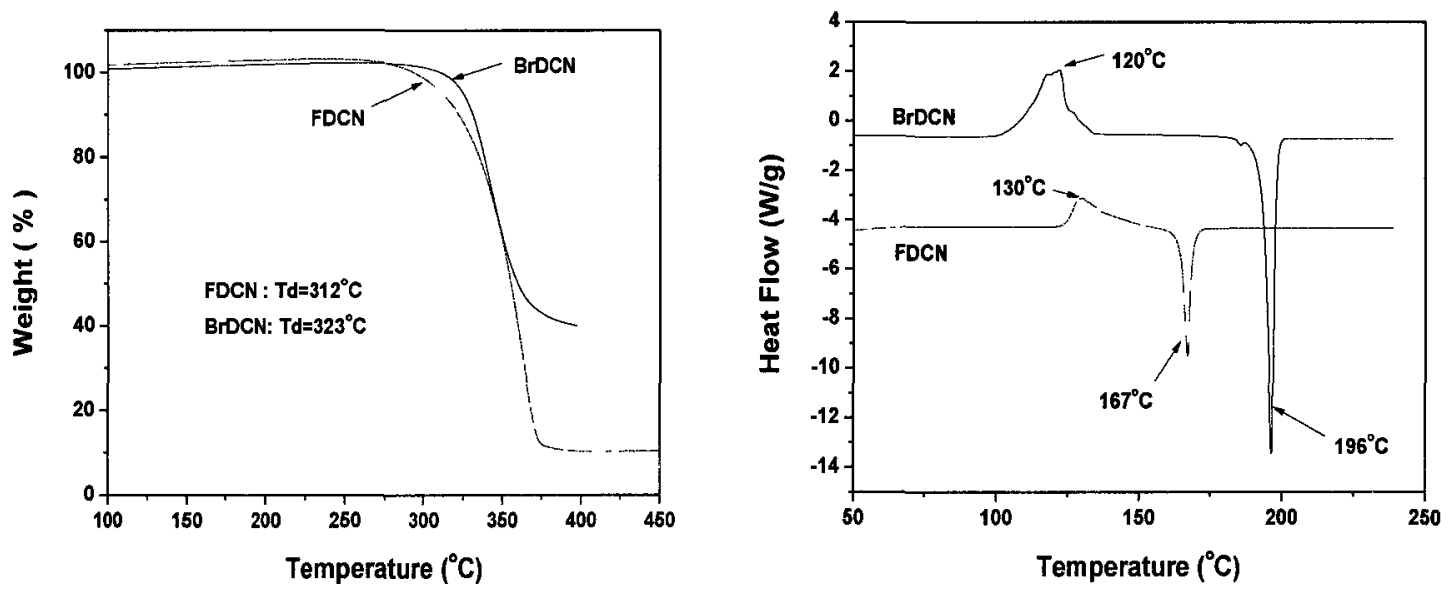

Figure 2.5. TGA (left) and DSC (right) traces of FDCN and BrDCN.

\subsubsection{Synthesis and Characterizations of PpQDM Chromophores}




\subsubsection{Synthesis of PpQDM Chromophores}

Using a strong electron-withdrawing group, 3CNQ, as the acceptor and dipyridylium with the different functional groups as the donor moieties, a new series of PpQDM chromophores were synthesized. The incorporation of a bulky side group is deemed to reduce the molecular symmetry and alleviate anti-parallel aggregation as well as improve solubility of these highly dipolar molecules. For further incorporation of the resulting chromophores into a polymer, (either by doping or grafting) and for comparison with the PQDM and PeQDM, the dipyridylium salts should be functionalized with some simple alkyl, benzyl and hydroxyl groups. In selecting the bromo alcohols, the rigidity and length of the spacer between the hydroxyl group and the core structure of PpQDM are considered and a flexible spacer (e.g., 3-carbon aliphatic chain) was proved to be appropriate. Using 4, 4'-dimethyl-2, 2'-dipyridyl as a starting material, the functionalized dipyridylium salts were typically prepared by refluxing the dipyridyl with corresponding alkyl halides at a high yield (Scheme 2.5).

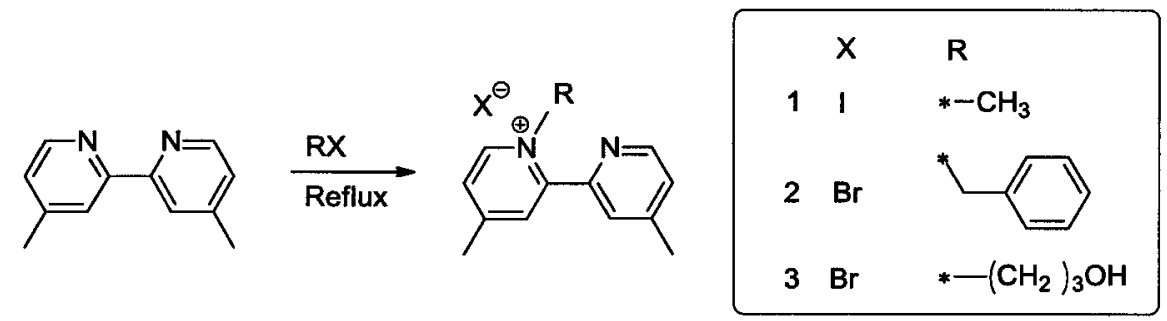

Scheme 2.5. Synthesis of dipyridylium halides.

To improve the synthesis of PpQDM chromophores, the reaction conditions were studied. First, lithium TCNQ salt was prepared by reaction of TCNQ and lithium iodide according to the literature method. ${ }^{22}$ Using 2 molar equivalent of lithium TCNQ adduct, one molar equivalent of dipyridylium halide in methanol, and in presence of 1- 
piperidineethanol as the base, three target PpQDM chromophores (PpQDM-Met, PpQDM-Ben, and PpQDM-C3OH) were successfully obtained in yields of $45-65 \%$ (Scheme 2.6).

The reaction was monitored by UV-Vis spectra of aliquots of the reaction mixture in DMF (Figure 2.6). The very intense peaks of lithium TCNQ salt at $422 \mathrm{~nm}, 747 \mathrm{~nm}$ and $848 \mathrm{~nm}$ decreased gradually when the dipyridylium halides and the base catalyst were added; the charge transfer peak of the resulting chromophore at around $690 \mathrm{~nm}$ started forming when half molar equivalent of catalyst was added, then it became much stronger than that of the radical anion peaks of TCNQLi after the other half of the catalyst was added.

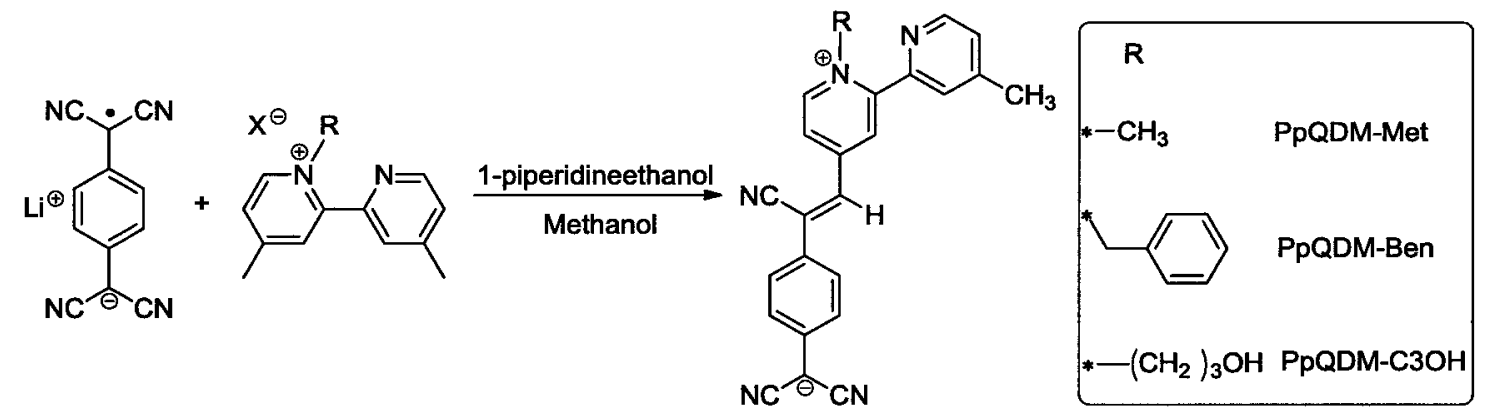

Scheme 2.6. Synthesis of PpQDM chromophores.

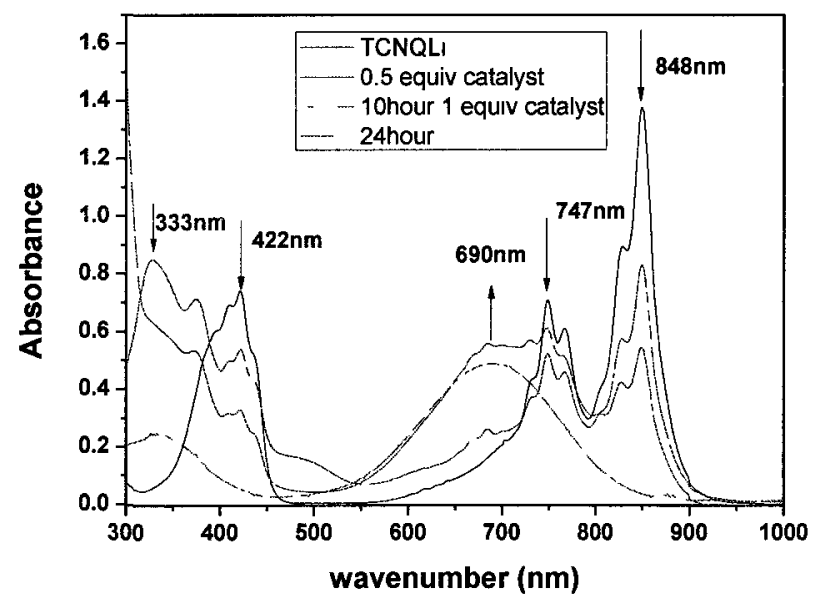

Figure 2.6. The formation of PpQDM-C3OH monitored by UV-Vis spectroscopy. 


\subsubsection{Characterizations of PpQDM Chromophores}

All of the PpQDM chromophores were purified by simple filtration and washing with methanol, and the structures have been fully characterized by ${ }^{1} \mathrm{H} N M R,{ }^{13} \mathrm{C} N M R$, IR and HRMS spectrometry (Appendix B, Figure S2.13).

As shown in IR spectrum of PpQDM-Met (Figure 2.7), two different stretching frequencies of the cyano groups were found. The first one at $2226 \mathrm{~cm}^{-1}$, belongs to the vinylcyano group which connects to the central carbon-carbon double bond, as in TCNQ $\left(v_{\mathrm{CN}}=2226 \mathrm{~cm}^{-1}\right)$; another group was at low wavenumber $\left(2173 \mathrm{~cm}^{-1}\right.$ and $\left.2137 \mathrm{~cm}^{-1}\right)$ due to a large formal vicinal charge at the corresponding dicyanovinyl site, indicated a considerable charge separation in the ground state of PpQDM-Met. The smaller $v_{C N}$ frequency for PpQDM-Met might be due to the two fold aromatization (both donor and acceptor rings) gained from ICT.

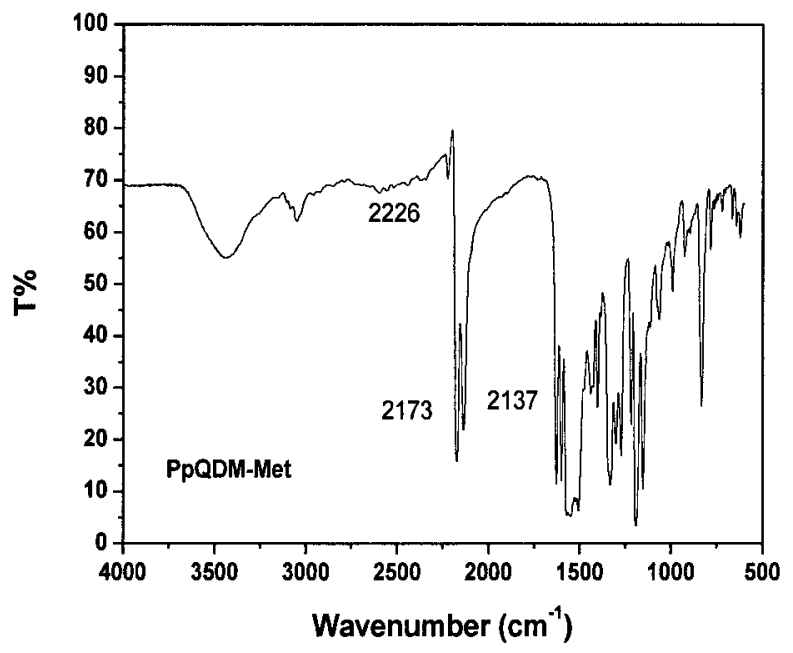

Figure 2.7. IR spectrum of $\mathrm{PpQDM}-\mathrm{Met}(\mathrm{KBr}$ pellet).

Figure 2.8 shows the ${ }^{1} \mathrm{H}$ NMR spectrum of PpQDM-Met with peaks assigned to the structure. ${ }^{1} \mathrm{H}$ NMR (2D COSY) spectrum of the aromatic protons of PpQDM-Met 
and ${ }^{13} \mathrm{C}$ NMR spectrum are shown in the Appendix B (Figures S2.11 and S2.12) and are consistent with the desired structure.

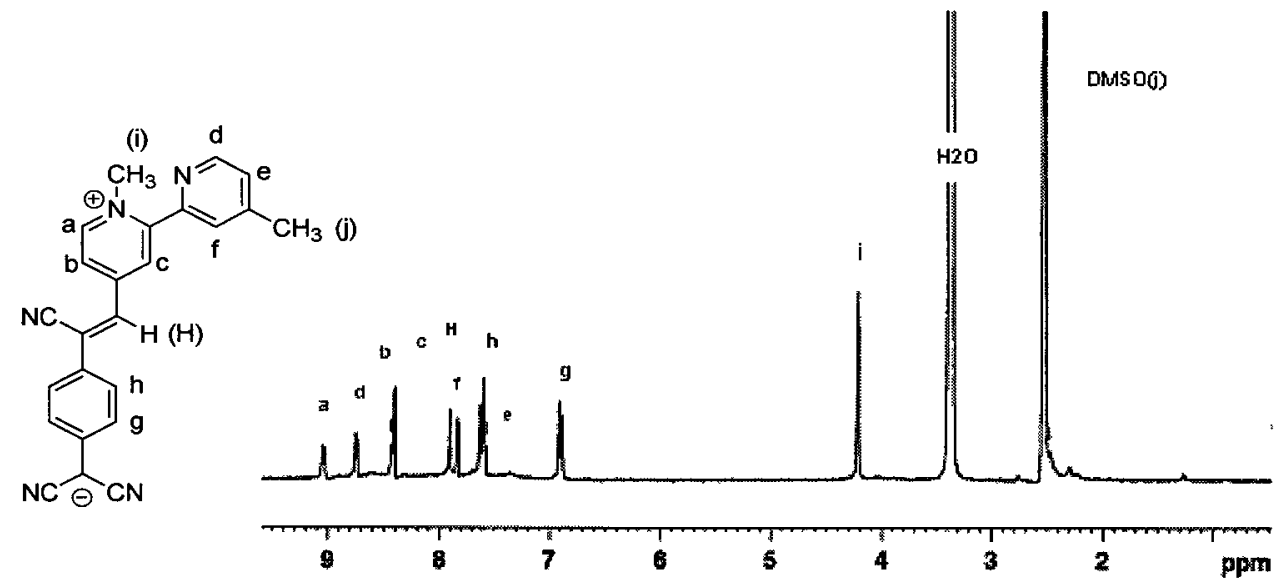

Figure 2.8. ${ }^{1} \mathrm{H}$ NMR spectrum of PpQDM-Met in DMSO- $\mathrm{d}_{6}$.

To illustrate the extent of ground state charge separation, selected ${ }^{1} \mathrm{H}$ NMR data of FDCN and FCNE, PpQDM-Ben and PeQDM-Ben, PpQDM-C3OH, PeQDM-C3OH, and PQDM-C3OH in DMSO are listed in Table 2.3.

Table 2.3. Selected ${ }^{1} \mathrm{H}$ NMR data for FDCN, FCNE, PpQDM-Ben, PeQDM Ben, PpQDM-C3OH, PeQDM-C3OH and PQDM-C3OH

\begin{tabular}{ccc}
\hline Compound & $\delta 1(\mathrm{ppm})^{\mathrm{a}}$ & $\delta 2(\mathrm{ppm})^{\mathrm{b}}$ \\
\hline FDCN & 7.81 & 3.92 \\
FCNE & 7.72 & 3.88 \\
PpQDM-Ben & 9.15 & 5.94 \\
PeQDM-Ben & 9.05 & 5.75 \\
PpQDM-C3OH & 9.06 & 4.62 \\
PQDM-C3OH & 8.94 & 4.57 \\
PeQDM-C3OH & 8.93 & 4.57 \\
\hline
\end{tabular}

${ }^{a}$ The ${ }^{1} \mathrm{H}$ NMR chemical shifts of protons on methine carbon of pyridium ring

${ }^{b}$ Protons of the methyl attached to the tertiary nitrogen 
The extent of downfield shifts of the ${ }^{1} \mathrm{H}$ NMR resonance of the protons on the methine carbon of pyridium ring is in accordance with the findings from $\mathrm{CN}$ vibration analysis. Additionally, $\mathrm{H}_{\mathrm{g}}$ and $\mathrm{H}_{\mathrm{h}}$ exhibit signals at $6.87 \mathrm{ppm}$ and $7.58 \mathrm{ppm}$, the appearance of these proton signals in the aromatic region, downfield from the olefinic region, further supports the reduced quinoid character in the ground state, due to ICT aromatization.

In the expanded $\pi$-conjugated systems, the larger downfield shift of the methine protons in PpQDM-C3OH (9.06 ppm), PQDM-C3OH (8.94 ppm) and PeQDM-C3OH (8.93 ppm) suggests a significant degree of charge separation in the ground state, as compared to FDCN $(7.81 \mathrm{ppm})$ and FCNE $(7.72 \mathrm{ppm})$. The larger downfield shift of the methine protons in PpQDM-Ben (9.15 ppm) compared to that of PeQDM-Ben (9.05 ppm) indicates a greater degree of ground state charge separation due to the composition of stronger acceptor (3CNQ) and dipyridylium donor. Therefore, PpQDMs are expected to have larger $\mu \beta$ values than PeQDM chromophores and improve the NLO properties.

\subsubsection{Thermal Stability of PpQDM Chromophores}

The PpQDM chromophores are quite thermally stable, as measured by TGA, a high onset temperature for $5 \%$ weight loss was found at $310{ }^{\circ} \mathrm{C}, 304{ }^{\circ} \mathrm{C}$ and $283{ }^{\circ} \mathrm{C}$ for PpQDM-Met, PpQDM-Ben and PpQDM-C3OH, respectively (Figure 2.9). PpQDM-Met containing methyl group shows the highest $\mathrm{T}_{\mathrm{d}}$, which is almost $25^{\circ} \mathrm{C}$ and $100{ }^{\circ} \mathrm{C}$ higher than that of PQDM and PeQDM, respectively. The enhanced thermal stability of PpQDM chromophores might be ascribed to the addition of the methyl pyridine ring on the aromatic donor. Such a thermal stability of PpQDM meets the requirements for most material processing and device fabrications. 


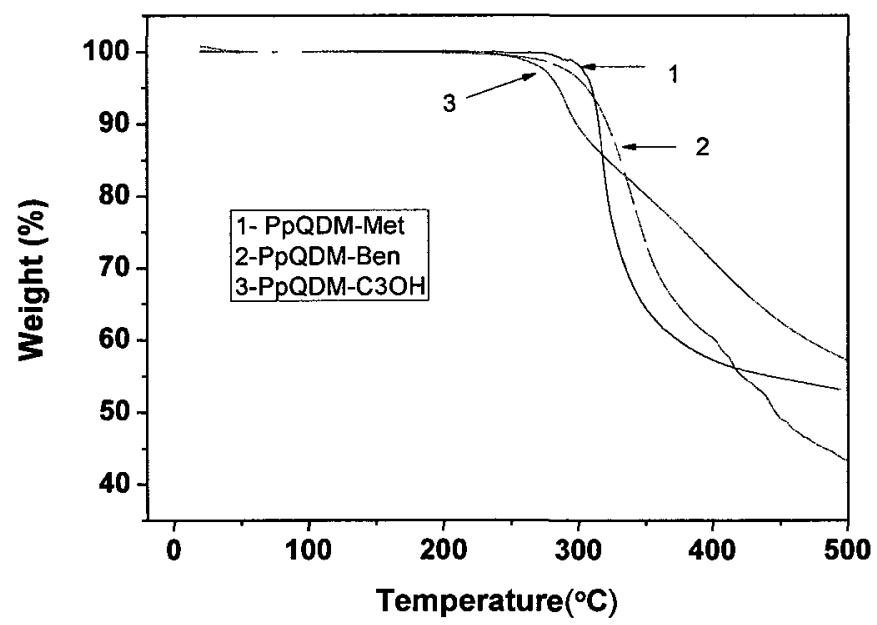

Figure 2.9. TGA trace of PpQDM-Met in nitrogen with a heating rate of $10{ }^{\circ} \mathrm{C} \mathrm{min}-1$.

\subsubsection{UV-Vis-NIR Absorption}

The absorption characteristics of FDCN and PpQDM chromophores were measured in DMF (Figure 2.10). All of the two series of chromophores exhibit intense optical absorption in the UV-Vis-NIR region with extinction coefficients as large as $33,300 \mathrm{M}^{-1} \mathrm{~cm}^{-1}$.
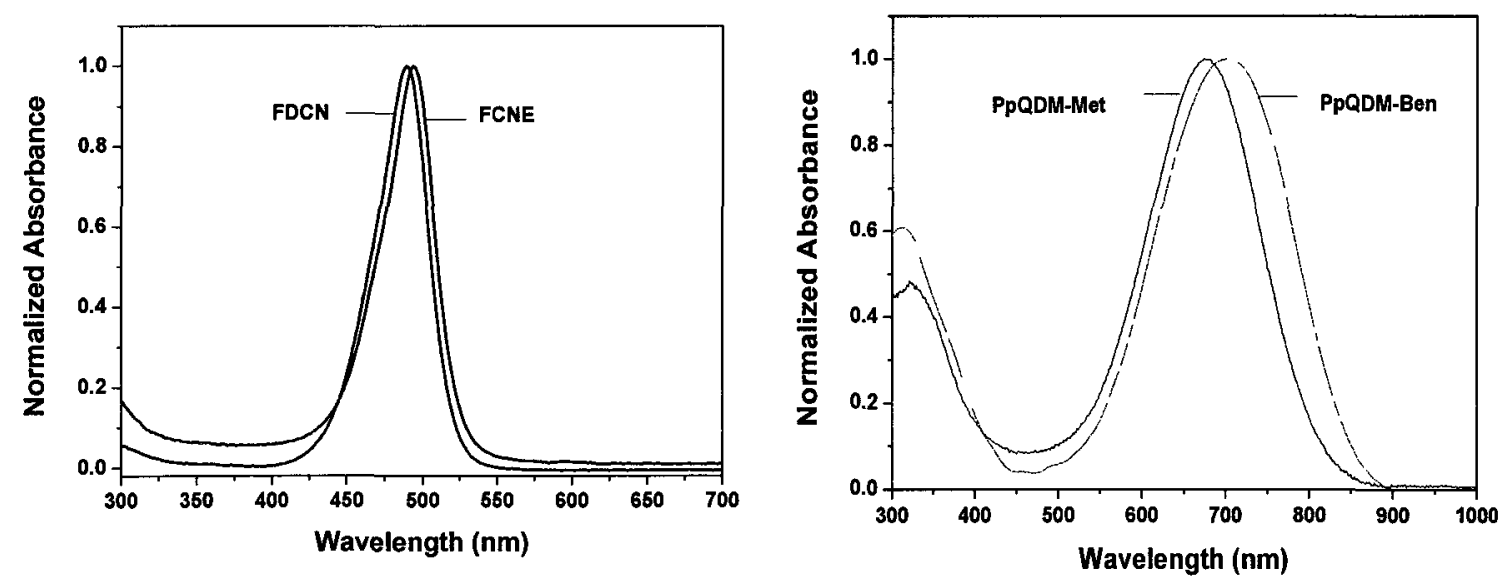

Figure 2.10. Optical absorption spectra of FDCN (left) and PpQDM (right) chromophores in DMF solution. 
The FDCN and FCNE optical absorption spectra are selected and show maximum absorption wavelength $\left(\lambda_{\max }\right)$ at $489 \mathrm{~nm}$ and $493 \mathrm{~nm}$, respectively, assignable to ICT excitation in Figure 2.10 (left), pyridinium intra-subfragment excitation is expected to be at higher energy ${ }^{23}$ and overlap with the solvent cut-off.

In the UV spectral region (Figure 2.10 right), the PpQDM spectrum also features an intense band at $330 \mathrm{~nm}$, assigned to pyridinium and phenyl subfragment high-energy intra-ring excitations; the strong long-wavelength absorption band at $680 \mathrm{~nm}$ (PpQDMMet) and $690 \mathrm{~nm}(\mathrm{PpQDM}-\mathrm{Ben})$ with a molar extinction coefficient of 26,000-27,000 $\mathrm{mol}^{-1} \mathrm{~cm}^{-1}$ is due to $\mathrm{CT}$ from the negatively charged dicyanomethanide group to the positively charged nitrogen in pyridium. ${ }^{24} \mathrm{CT}$ band of PpQDM-Met blue shifts $(10 \mathrm{~nm})$ compared to bulky phenyl-functionalized PpQDM-Ben due to stronger antiparallel centrosymmetric dimer formation as has also been reported in merocyanine dye aggregation studies. ${ }^{20}$ All of the PpQDM chromophores are readily soluble in polar aprotic solvents such as DMF, DMSO and NMP, but moderately soluble in polar solvents such as ACN, methanol and acetone and essentially insoluble in less polar solvents such as chloroform, THF and benzene.

\subsubsection{Negative Solvatochromism}

To investigate the solvatochromism of FDCN and PpQDM chromophores, the maximum optical absorption wavelengths of FDCNs and PpQDMs in various solvents were measured and the selected results are summarized in Table 2.4, while representative absorption spectra of PpQDM-Met are presented in Figure 2.11. 
Table 2.4. Optical absorption $\left(\lambda_{\max }, \mathrm{nm}\right)$ data for FDCN and PpQDM chromophores

\begin{tabular}{ccccccc}
\hline \multirow{2}{*}{ Compound } & \multicolumn{7}{c}{$\lambda_{\max }(\mathrm{nm})$} \\
\cline { 2 - 7 } & THF & Acetone & Methanol & DMF & ACN & DMSO \\
& $7.6^{\mathrm{a}}, 37.4^{\mathrm{b}}$ & $20.7,42.2$ & $32.7,55.4$ & $36.7,43.2$ & $37.5,45.6$ & $46.7,45.1$ \\
\hline FDCN & 495 & 487 & 480 & 485 & 483 & 484 \\
FCNE & 502 & 495 & 486 & 493 & 490 & 492 \\
PpQDM-Met & 810 & 713 & 647 & 680 & 673 & 655 \\
PpQDM-Ben & 830 & 737 & 678 & 691 & 689 & 683 \\
\hline
\end{tabular}

${ }^{a}$ Solvent Dielectric Constant ( $\varepsilon$ ) (Lide D. R. Handbook of chemistry and physics 76 th edition, P8-64, 1995-1996) ${ }^{\mathrm{b}}$ Reichardt $\mathrm{E}_{\mathrm{T}}{ }^{\left({ }^{(3)}\right.}$ values

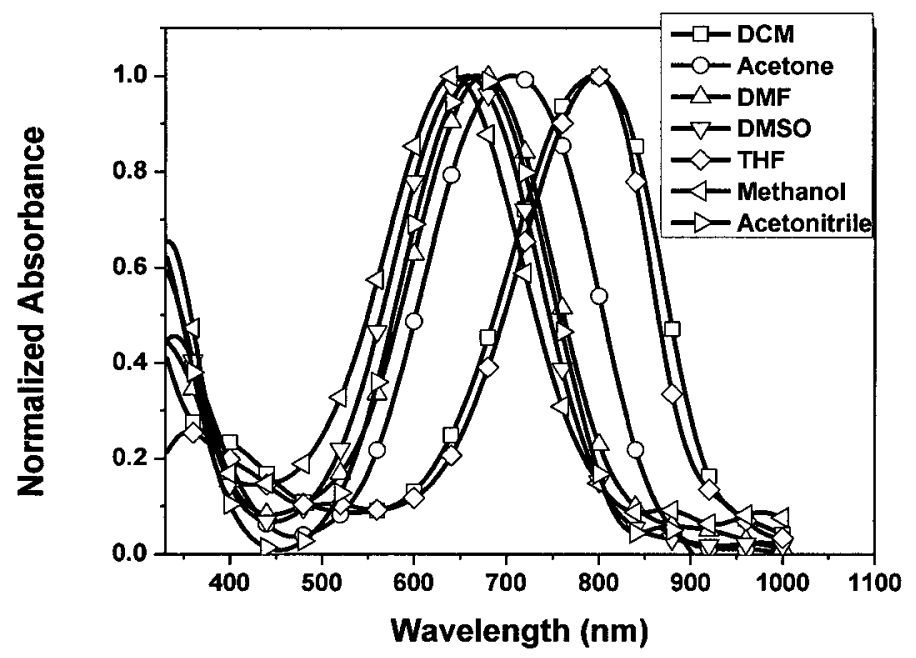

Figure 2.11. UV-Vis-NIR spectra of PpQDM-Met in different solvents.

In this experiment the relative polarity of the solvents was firstly determined based on their dielectric constants $(\varepsilon)$ : THF $<$ Dichloromethane (DCM) $<$ Acetone $<$ Methanol $<$ DMF $<$ ACN $<$ DMSO. The UV-Vis-NIR absorptions of PpQDM-Met in these solvents show the corresponding blue shift with increasing solvent polarity, with the exception of methanol. Considering solute/solvent interactions, another empirical parameter of solvent polarity, $\mathrm{E}_{\mathrm{T}}{ }^{(30)}$ was employed to determine the relative polarity of the solvents. $E_{T}{ }^{(30)}$ is a well selected strongly solvent-dependent spectral absorption 
parameter, $^{25}$ and the values are based on the negative solvatochromic betaine dye (pyridinium N-phenolate ) as a probe molecule (Structure in Chapter 1, Table 1.4), and they are defined as the molar electronic transition (ET) energy of the dissolved dye. By using the $\mathrm{E}_{\mathrm{T}}{ }^{(30)}$ parameter as the index of solvent polarity, the above solvents have a polar order: $\mathrm{THF}<\mathrm{DCM}<$ Acetone $<\mathrm{DMF}<\mathrm{ACN}<\mathrm{DMSO}<$ Methanol and the order is consistent with the UV-Vis-NIR spectral results of PpQDM-Met.

From the solvatochromism data presented in Table 2.4 and Figure 2.11, two major points are apparent, 1) the direction of the solvatochromic shift and 2) the magnitude of the solvatochromic shift $\left(\Delta \lambda_{\max }\right)$. It can be seen that the CT band of PpQDM-Met exhibits strong negative solvatochromic effect - a large blue shift with increasing solvent polarity (the spectra were recorded in the concentration range where aggregation is not significant). The CT band of PpQDM-Met is blue shifted from $810 \mathrm{~nm}$ in the less polar THF to $647 \mathrm{~nm}$ in the polar methanol. This represents a shift of $\sim 163 \mathrm{~nm}$, and is comparable to the largest solvatochromic effects reported in heavily studied merocyanine derivatives, ${ }^{20}$ approximately a third that of the Reichardt betaine dye. ${ }^{25}$ The negative solvatochormism result indicates that the charge-separated resonance form of PpQDMMet is more dominant in the ground state than the excited state $\left(\mu_{\mathrm{g}}>\mu_{\mathrm{e}}\right)$ (Scheme 2.7). Consequently, greater stabilization of the zwitterionic form occurs in high polarity solvents than in low polarity solvents, leading to higher energy absorption maximum in high polarity solvents. FDCN chromophores also show a blue shift in their absorption maxima in going from non-polar to polar solvents and are therefore good examples of zwitterionic chromophores, which is consistent with the conclusion obtained from IR and X-ray crystallographic results. 


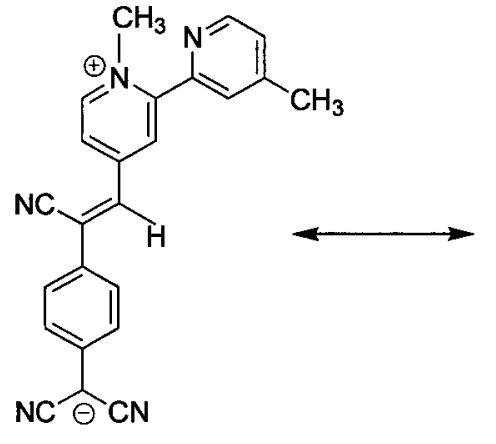

Zwitterionic Form (Ground state)<smiles>Cc1ccnc(C2=CC(=CC(C#N)=c3ccc(=C(C#N)C#N)cc3)C=CN2C)c1</smiles>

Neutral Form (Exited state)

Scheme 2.7. Resonance structures of PpQDM-Met.

With respect to the second observation, the magnitude of the solvatochromic shift in the PpQDM chromophores (on average $157 \mathrm{~nm}$ ) is larger than the solvatochromic shift in the FDCN chromophores (on average $15 \mathrm{~nm}$ ). This can be explained by the magnitude of the gain in resonance stabilization energy upon aromatization of the two donor and acceptor units. The gain in aromaticity for the FDCN chromophores is expected to be less than in the case of PpQDMs due to the combination of a weaker acceptor and shorter conjugated bridge. Therefore, in comparison to the charge-separated forms of PQDM (a blue shift as large as $125 \mathrm{~nm}$ ), PpQDMs chromophores are more stabilized in high polarity media, meaning the first hyperpolarizabilities and dipole moments of the PpQDM chromophores will be larger than those observed for the PQDM chromophores.

Few TCNQ-based chromophores have been described in terms of their solvent polarity indicating abilities. DEMI was extensively studied in terms of solvatochromic effects and shows a very limited response when compared to the Reichardt betaine dye. ${ }^{26}$ Thus PpQDMs appear to be one of the novel $\mathrm{D}^{\delta+}-\pi-\mathrm{A}^{\delta-}$ solvatochromic systems. 


\subsubsection{Fluorescence Properties of FDCN and PpQDM Chromophores}

The sensitivity of betain dyes to solvent polarity is exceptionally high; however, they are not fluorescent. Polarity-sensitive fluorescent dyes offer distinct advantages especially in biological studies. The fluorescence properties of FDCN and PpQDM chromophores in DMF were studied, and the results are summarized in Table 2.5 with the optimal concentration for PL measurement to be $2 \times 10^{-5} \mathrm{M}$. The emission spectra of dilute solutions of FDCN and PpQDM-Met chromophores excited at $470 \mathrm{~nm}$ and $670 \mathrm{~nm}$ are presented in Figure 2.12. The strongest emission bands of FDCN and PpQDM-Met have maxima at $530 \mathrm{~nm}$ and $875 \mathrm{~nm}$, respectively. Further, the fluorescence spectra associated with the lowest excited singlet states display mirror image relationships to the corresponding absorption bands with Stokes shifts of between $45 \mathrm{~nm}\left(1388 \mathrm{~cm}^{-1}\right)$ and 195 $\mathrm{nm}\left(3277 \mathrm{~cm}^{-1}\right)$ which are typical of dipolar, monomeric molecules in polar solvents. ${ }^{27}$
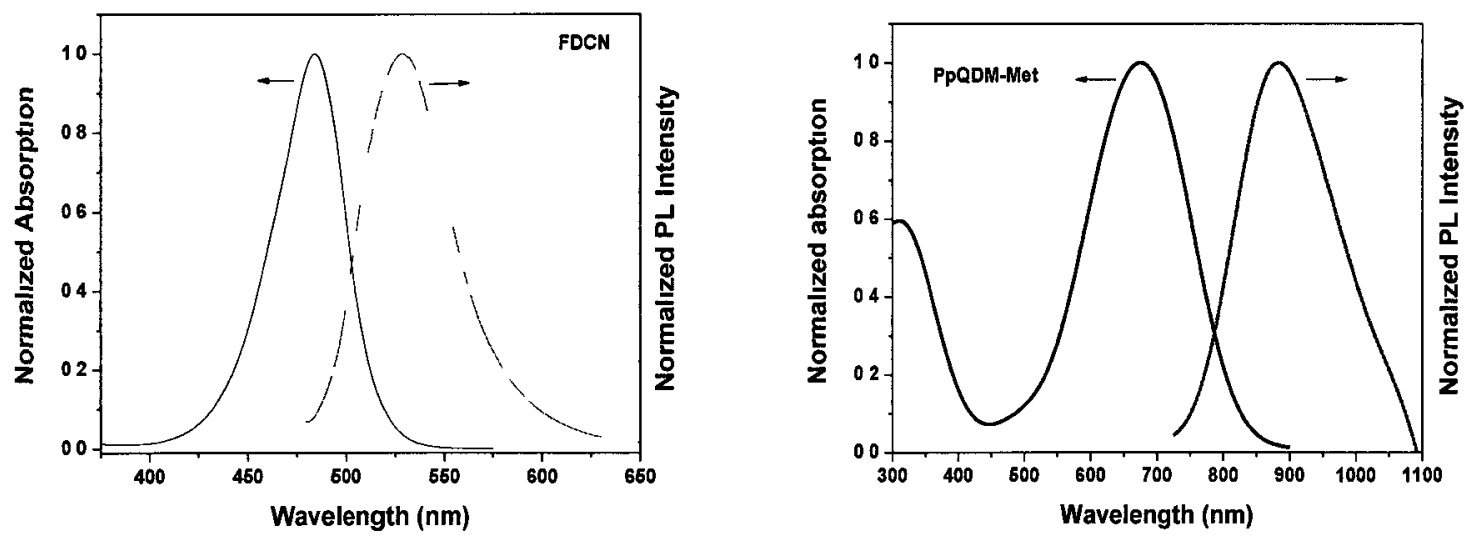

Figure 2.12. The normalized absorption and emission spectra of FDCN (left) and PpQDM-Met (right) in DMF.

Stokes shift (the gap between the maximum of the first absorption band and the maximum of the fluorescence spectrum, expressed in wavenumbers, $\Delta \bar{v}=\bar{v}_{a}-\bar{v}_{f}$ ) 
indicates the difference in the properties and the structure between the ground $\mathrm{S}_{0}$ state, and the first exited $S_{1}$ state. The values of Stokes shift of FDCN is relative smaller around $1760 \mathrm{~cm}^{-1}$ (shift between the maximum of emission and maximum of the absorption $\Delta \lambda=$ $\left.\lambda_{\mathrm{F}}-\lambda_{\mathrm{A}}=40 \sim 45 \mathrm{~nm}\right)$ than that of PpQDM which are in the $2950-3277 \mathrm{~cm}^{-1}$ region $(\Delta \lambda=$ $\left.\lambda_{\mathrm{F}}-\lambda_{\mathrm{A}}=190 \sim 195 \mathrm{~nm}\right)$, due to its shorter conjugated bridge and smaller relative dipole moment compared with PpQDMs. The larger Stokes shift of PpQDM is in accordance with other investigations on PQDM and PeQDM derivatives. Compared with other known zwitterionic compounds, such as DEMI, whose Stokes shift is only $1505 \mathrm{~cm}^{-1}(\Delta \lambda$ $=770-690=80 \mathrm{~nm})$, indicate that the dipyridium donor is a key determinant in making the PpQDM-Met into an extended delocalized $\pi$-electron system.

Table 2.5. Optical properties of the band of CT of FDCNs and PpQDMs in DMF

\begin{tabular}{ccccc}
\hline Compound & $\lambda_{\mathrm{A}}(\mathrm{nm})^{\mathrm{a}}$ & $\lambda_{\mathrm{F}}(\mathrm{nm})$ & $\lambda_{\mathrm{F}}-\lambda_{\mathrm{A}}(\mathrm{nm})$ & $\Phi_{\mathrm{F}}(\%)$ \\
\hline FDCN & 485 & $520^{\mathrm{b}}$ & 45 & $0.40^{\mathrm{d}}$ \\
FCNE & 493 & $533^{\mathrm{b}}$ & 40 & $0.27^{\mathrm{d}}$ \\
PpQDM-Met & 680 & $875^{\mathrm{c}}$ & 195 & $0.20^{\mathrm{e}}$ \\
PpQDM-Ben & 705 & $890^{\mathrm{c}}$ & 185 & $0.19^{\mathrm{e}}$ \\
\hline
\end{tabular}

${ }^{a}$ Measured in DMF at a concentration of $2 \times 10^{-5} \mathrm{M} .{ }^{b}$ Measured in DMF at a concentration of $2 \times 10^{-5} \mathrm{M}$. Excitation wavelength $=470 \mathrm{~nm}$. ${ }^{\mathrm{c}}$ Measured in DMF at a concentration of $2 \times 10^{-5} \mathrm{M}$. Excitation wavelength $=670 \mathrm{~nm}$. ${ }^{\mathrm{d}}$ Fluorescence quantum yield, relative to Rhodamin $6 \mathrm{G}\left(\Phi_{\mathrm{F}}=0.88\right.$ in Ethanol). ${ }^{\mathrm{e}}$ Fluorescence quantum yield, relative to IR-125 $\left(\Phi_{\mathrm{F}}=0.13\right.$ in DMSO).

In this work, both FDCN and PpQDM-Met as the representatives of the two new series of chromophores were evaluated as polarity-sensitive fluorescent dyes. In 2008, PeQDM-Ben was found to exhibit NIR photoluminescence at $\sim 860 \mathrm{~nm}$ in DMF solution when being excited at $650 \mathrm{~nm},{ }^{28}$ and it has potential application as a $\mathrm{pH}$ sensor. PpQDM chromophores show tuneable NIR fluorescence (e.g., 875 to $890 \mathrm{~nm}$ ) longer than that of 
PeQDM by changing the strength of donor through introducing different substituted groups on the nitrogen of the dipyridium ring, which indicated that the stronger charge transfer occurred in PpQDM than that in PeQDM, therefore, PpQDM were expected to show stronger fluorescence sensitivity to the polarity of the environment.

First, the sensitivity of the FDCN fluorescence to solvent polarity was investigated; the results are summarized in Table 2.6. As seen in Figure 2.13, a series of fluorescence spectra of FDCN show the blue shift with increasing solvent polarity, the spectral maxima extending from $524 \mathrm{~nm}$ (in methanol) to $539 \mathrm{~nm}$ (in 1,4- dioxane). This is the first report of zwitterionic chromophores showing potential application as polaritysensitive fluorescent dyes in the visible region.

Table 2.6. Optical properties of the CT band of FDCN in different solvents

\begin{tabular}{cccccc}
\hline Solvent & $\begin{array}{c}\text { Dielectric } \\
\text { constant }(\varepsilon)\end{array}$ & $\begin{array}{c}\lambda_{\mathrm{A}} \\
(\mathrm{nm})\end{array}$ & $\begin{array}{c}\varepsilon \\
\left(\mathrm{Lmol}^{-1} \mathrm{~cm}^{-1}\right)\end{array}$ & $\begin{array}{c}\lambda_{\mathrm{F}} \\
(\mathrm{nm})\end{array}$ & $\begin{array}{c}\lambda_{\mathrm{F}}-\lambda_{\mathrm{A}} \\
(\mathrm{nm})\end{array}$ \\
\hline 1,4-Dioxane & 2.2 & 498 & 75,498 & 539 & 41 \\
THF & 7.6 & 495 & 97,380 & 538 & 43 \\
DCM & 8.9 & 498 & 102,300 & 535 & 37 \\
Acetone & 20.7 & 487 & 96,224 & 531 & 44 \\
Methanol & 32.7 & 480 & 104,540 & 520 & 44 \\
DMF & 36.7 & 484 & 64,400 & 529 & 45 \\
ACN & 37.5 & 483 & 95,340 & 526 & 43 \\
DMSO & 46.7 & 485 & 106,320 & 528 & 43 \\
\hline
\end{tabular}




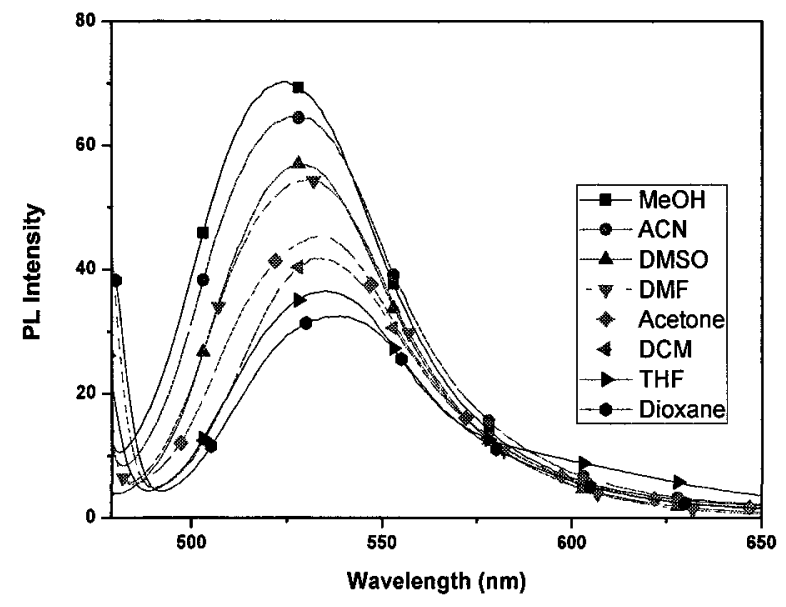

Figure 2.13. Fluorescence spectra of FDCN solutions excited at $460 \mathrm{~nm}$. The successive maxima correspond to methanol, ACN, DMSO, DMF, acetone, DCM, THF and 1, 4dioxane. The heights do not reflect relative fluorescence yield.

Compared with FDCN, PpQDM-Met offers outstanding sensitivity of fluorescence to the changes in solvent polarity. The fluorescence spectra show the remarkable blue shift with increasing solvent polarity, which extends from $840 \mathrm{~nm}$ (in methanol) to $930 \mathrm{~nm}$ (in DCM). Table 2.7 shows the maximum fluorescence and absorption data of PpQDM-Met in various solvents. The shift of the absorption peaks with solvent polarity $\left(\Delta \lambda_{A}=148 \mathrm{~nm}\right.$ from THF to methanol) are more pronounced than the shifts of emission peaks $\left(\Delta \lambda_{\mathrm{F}}=75 \mathrm{~nm}\right)$. This indicates that $\mu_{\mathrm{g}}>\mu_{\mathrm{e}}$, dipole moment of the chromophore decreases upon excitation. The increase in the Stokes shift from 1649 $\mathrm{cm}^{-1}$ in THF to $3693 \mathrm{~cm}^{-1}$ in DMSO is very large. The increased Stokes shift in polar solvents can be attributed to the higher degree of solvent stabilization effect on the ground state energy than on the excited state. This indicates that the ground state dipole moment of PpQDM-Met is significantly larger than the one in the excited state, leading to pronounced solvatochromism of fluorescence. The larger shift between the emission and absorption of PpQDM in polar solvents offers the most important additional 
advantage for PpQDM chromophores - application as a novel environmentally sensitive single molecule fluorophore. ${ }^{29}$

As shown in Table 2.7, the emission yields of PpQDM-Met shows large variations depending strongly on the polarity of the solvent, with values in the range of 0.058-0.7. Figure 2.14 presents the dependence of the quantum fluorescence yield on the dielectric constant of the solvents. The maximum yields are found in solvents of higher polarity, with a several-fold decrease in low polar solvents.

Table 2.7. Optical properties of the CT band of PpQDM-Met in different solvents

\begin{tabular}{cccccccc}
\hline Solvent & $\begin{array}{c}\text { Dielectric } \\
\text { constant }(\varepsilon)\end{array}$ & $\begin{array}{c}\lambda_{\mathrm{A}} \\
(\mathrm{nm})\end{array}$ & $\begin{array}{c}\lambda_{\mathrm{F}} \\
(\mathrm{nm})\end{array}$ & $\begin{array}{c}\lambda_{\mathrm{F}}-\lambda_{\mathrm{A}} \\
(\mathrm{nm})\end{array}$ & $\begin{array}{c}\text { Stokes } \\
\text { shift }\left(\mathrm{cm}^{-1}\right)\end{array}$ & $\begin{array}{c}\Phi_{\mathrm{F}} \\
(\%)^{\mathrm{a}}\end{array}$ & $\Delta f^{\mathrm{b}}$ \\
\hline THF & 7.6 & 795 & 915 & 120 & 1649 & 0.058 & 0.210 \\
DCM & 8.9 & 805 & 930 & 125 & 1669 & 0.073 & 0.219 \\
Acetone & 20.7 & 713 & 885 & 172 & 2726 & 0.088 & 0.287 \\
Methanol & 32.7 & 647 & 840 & 193 & 3550 & 0.137 & 0.308 \\
DMF & 36.7 & 680 & 875 & 195 & 3277 & 0.20 & 0.276 \\
ACN & 37.5 & 673 & 870 & 197 & 3356 & 0.32 & 0.304 \\
DMSO & 46.7 & 655 & 864 & 209 & 3693 & 0.70 & 0.265 \\
\hline
\end{tabular}

${ }^{a}$ Fluorescence quantum yield, relative to IR-125 $\left(\Phi_{\mathrm{F}}=0.13\right.$ in DMSO).

${ }^{\mathrm{b}}$ The orientational polarizability of the solvents.

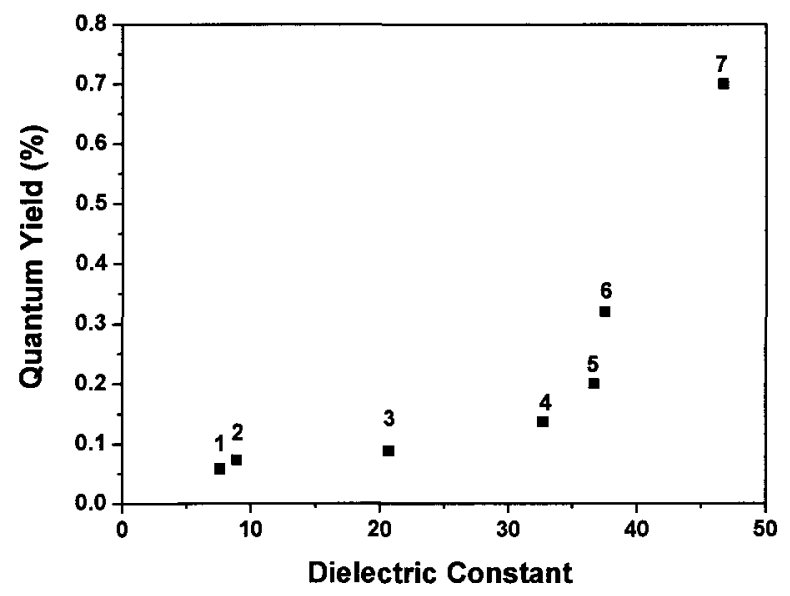

Figure 2.14. Fluorescence quantum yields $\left(\Phi_{\mathrm{F}}\right)$ of PpQDM-Met vs. the solvent dielectric constant ( $\varepsilon$ ) THF (1), DCM (2), acetone (3), MeOH (4), DMF (5), ACN (6), DMSO (7). 
The Stokes shifts against the orientational polarizability of the solvent was also shown in Table 2.7 , the orientational polarizability of the solvent is defined by eq. $1,{ }^{30}$ where $n$ is the refractive index of the solvent and $\varepsilon$ is its dielectric constant. Inspection of Table 2.7 shows that at similar values of $\Delta f$ (compare methanol and $\mathrm{ACN}$, both close to $\Delta f=0.3$ ), the aprotic solvent $\mathrm{ACN}$ produce considerably smaller Stokes shifts than those which can form a hydrogen bond by acting as proton donors. Similar effects have been noted for other studied fluorophores. ${ }^{31}$

$$
\Delta f=\frac{\varepsilon-1}{2 \varepsilon+1}-\frac{n^{2}-1}{2 n^{2}+1}
$$

Solvatochromic shift of the emission spectra is often applied to determine the dipole moment difference $\Delta \mu$ between the first excited electronic state and the ground state of chromophores. The larger $\Delta \mu$ of PpQDMs than PQDMs and PeQDMs were proven by the larger solvatochromic shift of their emission spectra. The fluorescent property of PpQDMs will be further discussed in Chapter 5, together with NIR fluorescent probes application.

\subsubsection{Dipole Moment and Molecular Hyperpolarizability}

The dipole moments of a molecule in the ground and excited states are different due to changes in electron densities in these states. A change in solvent is accompanied by a change in polarity, dielectric constant or a change in polarizability of the surrounding medium. Thus, a change of solvent affects the ground and excited states differently. A prior knowledge of the dipole moments of electronically excited species is often useful in the design of NLO materials. 
The dipole moment of FDCN and FCNE were measured by the most common method based on dielectric and refractive index measurements according to Debye's theory. The changes in the dielectric constant and the refractive index of solutions of the chromophores in nonpolar solvent, chloroform, are measured and a dipole moment of 21.6 D for FDCN and 11.39 D for FCNE were obtained.

The calculation of the dipole moment of PpQDM chromophores was performed as a result of their lower solubility in nonpolar solvents. Structures of these chromophores were sketched and energy minimized using PM6 Method. The dipole moments were calculated using B3LYP/6-31+G (d) method and the results were shown in Table 2.8.

To be useful for EO applications, potential chromophores need to possess large NLO responses $\left(\mu \beta_{0}\right)$ greater than $2000 \times 10^{-48}$ esu. ${ }^{32}$ Molecular first hyperpolarizability measurements can be carried out by a number of techniques including EFISH, HyperRayleigh scattering (HRS), etc. Among of these techniques, HRS is the most straight forward techniques, since it is based on the incoherent SHG obtained directly from randomly oriented chromophores in a dilute solution.

As a collaborative work, HRS measurements were carried out on two chromophores from the PQDM and PeQDM series ${ }^{10,12}$ by a physics group at the University Antwerpen in Belgium, yielding extremely large dynamic first hyperpolarizabilities of $\beta_{z z z}=1865 \times 10^{-30} \mathrm{esu}$ for PQDM-Met and $\beta_{z z z}=1797 \times 10^{-30}$ esu for PeQDM-Ben. These values correspond to $\beta_{0}$ value of 595 and $670 \times 10^{-30} \mathrm{esu}$, respectively, which are in agreement with theoretical predictions. In this work, dynamic $\beta$ values of FDCN and FCNE were measured by the same HRS method in DMF solution at $1.07 \mu \mathrm{m}$ wavelength, and are represented as the $\beta_{z z z}$ component, assuming all other 
components are negligible (i.e. an idealized linear chromophore is assumed). The $\beta$ values of PpQDM chromophores were calculated from Gaussian 98, results based on the above experimental data and known standards (e.g., DEMI, $27 \mathrm{D}, \mu \beta_{0}$ value of $9500 \times$ $\left.10^{-48} \mathrm{esu}\right){ }^{33}$ All of the experimental and theoretical results are summarized in Table 2.8 .

Table 2.8. Physical properties of FDCN and PpQDM chromophores, in comparison to the PQDM and PeQDM chromophores

\begin{tabular}{clcc}
\hline Chromophore & \multicolumn{1}{c}{$\mu(\mathrm{D})$} & $\beta\left(\times 10^{-30} \mathrm{esu}\right)$ & $\beta_{0}\left(\times 10^{-30} \mathrm{esu}\right)$ \\
\hline FDCN & $21.6^{\mathrm{a}}$ & $-320^{\mathrm{c}}$ & $45^{\mathrm{d}}$ \\
FCNE & $11.39^{\mathrm{a}}$ & $-495^{\mathrm{c}}$ & $57^{\mathrm{d}}$ \\
PpQDM-Met & $50.0^{\mathrm{b}}$ & $-1918^{\mathrm{b}}$ & 697 \\
PpQDM-Ben & $47.0^{\mathrm{b}}$ & $-1942^{\mathrm{b}}$ & 752 \\
PQDM-Met & $40.5^{36}$ & $-1865^{\mathrm{c}}$ & $595^{5}$ \\
PeQDM-Ben & -- & $-1797^{\mathrm{c}}$ & $670^{12}$ \\
\hline
\end{tabular}

${ }^{a}$ experimental value measured in $\mathrm{CHCl}_{3} ;{ }^{b}$ calculated value; ${ }^{\mathrm{c}}$ experimental value measured in DMF at $1070 \mathrm{~nm} ;{ }^{\mathrm{d}} \beta_{0}$ (static hyperpolarizability) was derived from the simple two-level model as described in eq. $2:{ }^{35}$

$$
\beta=\beta_{0} \frac{\lambda^{4}}{\left(\lambda^{2}-\lambda_{\max }^{2}\right)\left(\lambda^{2}-4 \lambda_{\max }^{2}\right)}
$$

$\lambda$ is the wavelength of the optical field $(1.07 \mu \mathrm{m})$ and $\lambda_{\max }$ is the wavelength of the CT band. PQDM-Met: $\lambda_{\max }=654 \mathrm{~nm}$ in DMF; ${ }^{11}$ PeQDM-Ben: $\lambda_{\max }=691 \mathrm{~nm}$ in $\mathrm{DMF}^{13}$; PpQDM-Met: $\lambda_{\max }=680 \mathrm{~nm}$ in DMF and PpQDM-Ben: $\lambda_{\max }=690 \mathrm{~nm}$ in DMF.

The results from Table 2.8 show that PpQDM chromophores have larger hyperpolarizabilities than PQDM and PeQDM chromophores. The $\beta$ values of PpQDM chromophores are almost 4 times higher than that of FDCNs, which are attributed to an increase in conjugation leading to a gain in stability from the aromatized negatively charged acceptor; when compared to DEMI $\left(\mu \beta_{0}=9500 \times 10^{-48} \mathrm{esu}\right)$, the significant enhancement of hyperpolarizabilities (e.g., $\mu \beta_{0}=34,850 \times 10^{-48}$ esu for PpQDM-Met) are due to the stronger aromatic donor. By taking the dipole moment of PpQDM-Met $(\mu=50$ 
D), an extraordinary large $\mu \beta$ value of $-95,900 \times 10^{-48}$ esu was obtained, which is much larger than that of the benchmark chromophore, FTC $\left(\mu \beta=18,000 \times 10^{-48}\right.$ esu).

\subsubsection{Acid Sensitivity}

In the previous study, PQDM and PeQDM were found to be sensitive to acids such as $\mathrm{H}_{2} \mathrm{SO}_{4}, \mathrm{HCl}$ and acetic acid, but fairly stable in basic media at room temperature. Due to the longer "push-pull" backbone, PpQDMs show a stronger CT absorption band at $680 \mathrm{~nm}$ to $690 \mathrm{~nm}$ in DMF (Figure 2.15) and give a bright blue color. The pronounced negative halochromism of PpQDM-Met can be demonstrated by a test-tube experiment: upon addition of water, the absorption of PpQDM-Met in DMF shifted to shorter wavelengths and causes a distinct color change from blue $\left(\lambda_{\max }=680 \mathrm{~nm}\right)$ to purple $\left(\lambda_{\max }\right.$ $=580 \mathrm{~nm}, \Delta \lambda=-100 \mathrm{~nm})$ at a $\left(\mathrm{H}_{2} \mathrm{O} / \mathrm{DMF}\right)$ volume ratio of $5: 1$. When an excess of acid is present, the blue color solution of PpQDM-Met turned colorless immediately, the visible absorption band disappears on the protonation of the negative carbon atom, while the intra-ring excitation band at $330 \mathrm{~nm}$ is unaffected. Subsequent addition of DMF to the acid containing solution moved the absorption back to $680 \mathrm{~nm}$.

This reversible color-changing process is believed to be due to the protonation of the chromophore by acid and deprotonation by base at the acceptor moiety (Scheme 2.8). To further investigate the mechanism of the halochromism, P-PpQDM-Met (protonated PpQDM-Met) was prepared by introducing an excess of dry $\mathrm{HCl}$ to the solution of PpQDM-Met and precipitated from ethyl ether. 


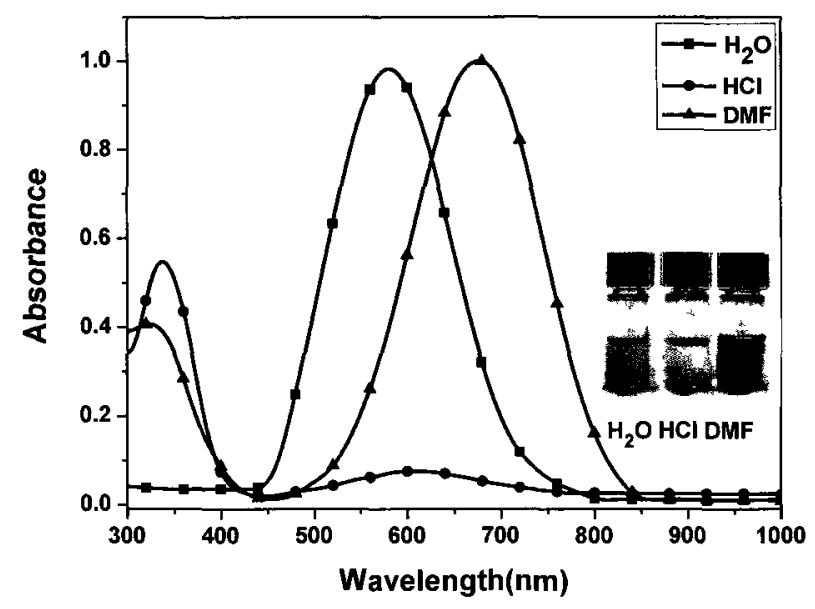

Figure 2.15. UV-Vis spectra of PpQDM-Met in DMF, $\mathrm{H}_{2} \mathrm{O}$ and $\mathrm{HCl}$.<smiles>CO[P+]([O-])(O)[C@H](C#N)c1ccc(/C=C/c2cc[n+](C)c(-c3cc(C)ccn3)c2)cc1</smiles><smiles>CO[P+](C)([O-])C(C#N)c1ccc(/C=C/c2cc[n+](C)c(-c3cc(C)ccn3)c2)cc1</smiles>

Scheme 2.8. Protonation of PpQDM-Met and deprotonation of P-PpQDM-Met.

The results from Figure 2.16 show that the position and intensity of $v_{C \equiv N}$ at the acceptor of PpQDM-Met changed noticeably after protonation. Before protonation, $v_{\mathrm{C} \equiv \mathrm{N}}$ was at a lower frequency $\left(2173 \mathrm{~cm}^{-1}\right.$ and $\left.2137 \mathrm{~cm}^{-1}\right)$ with high intensity due to the adjacent negative carbon center $-\mathrm{C}(\mathrm{CN})_{2}$; after protonation, $v_{\mathrm{C} \equiv \mathrm{N}}$ moved to higher frequency $\left(2248 \mathrm{~cm}^{-1}\right)$ with a much smaller intensity, which is typical for $\mathrm{C} \equiv \mathrm{N}$ group connected to the neutral saturated carbon atom. The vinylcyano group at the central part of the chromophore $\left(2226 \mathrm{~cm}^{-1}\right)$ did not show any difference after the protonation. This indicated that the negatively charged part of PpQDM-Met can function as a binding site 
to proton due to the presence of the negative charge. The strong dipyridium donor and $-\mathrm{C}$ $(\mathrm{CN})_{2}$ negative carbon center make PpQDM-Met a promising molecular probe to further investigate the spectra response upon binding with metal ions or neutral organic molecules. The application of this colorimetric and fluorescence molecular probe for sensitive and selective detection of metal ions and amino acids will be discussed in Chapter 5.

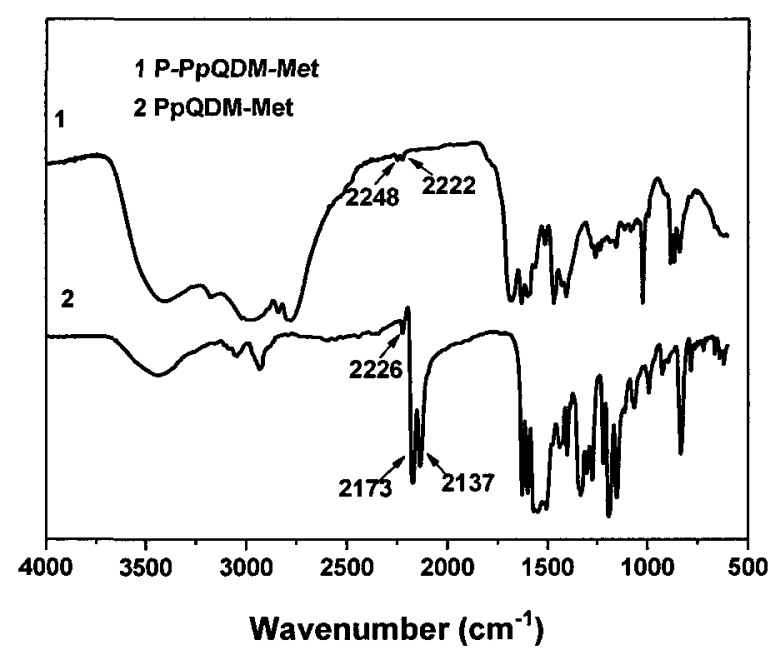

Figure 2.16. IR spectra of PpQDM-Met and P-PpQDM-Met.

\subsection{Conclusions}

Two series of novel zwitterionic chromophores FDCNs and PpQDMs have been designed and synthesized in moderate yields (40-60\%). Optimal synthetic conditions have been established for both series of chromophores. Hydroxyl-containing FDCN and PQDM chromophores have been synthesized for covalent coupling with polymers. X-ray crystallographic data, negative solvatochromism, IR and ${ }^{1} \mathrm{HNMR}$ and ${ }^{13} \mathrm{CNMR}$ data analysis confirm the highly charge-separated ground state of these two series of zwitterionic chromophores. These chromophores show good solubility in common organic solvents and high thermal stability. 
X-ray crystallographic data of FDCN demonstrated that incorporation of bulk groups onto central part of the chromophore is an effective strategy to minimize dipoledipole molecular interaction; higher solubility of FDCN in low polarity solvents makes it a good zwitterionic chromophore for experimental measurements of dipole moment and first hyperpolarizability, and these accurate experimental values were employed as internal standards for theoretical calculation for that of PpQDMs chromophores.

PpQDM chromophores with a stronger donor (dipyridylium), longer conjugation bridge and stronger acceptor $3 \mathrm{CNQ}$ were found to have large dipole moment and very large molecular hyperpolarizability, which demonstrates the great potential of this type of chromophores for NLO applications.

The solvent dependence of the fluorescence of PpQDM chromophores has been investigated. PpQDMs chromophores are NIR fluorescent with large Stokes shift (3839 $\mathrm{cm}^{-1}$ in polar solvent) and show high microenvironmental (e.g., $\mathrm{pH}$ and solvent polarity) sensitivity, which make them an interesting new class of NIR fluorescent polarity probes and promising candidates for NIR fluorescent chemosensor applications.

\subsection{Experimental Section}

Materials. TCNQ was purchased from Aldrich Chemical Canada. Lithium TCNQ salt was prepared according to the literature procedure. ${ }^{22}$ DMF was dried and distilled over $\mathrm{CaH}_{2}$ under an atmosphere of dry nitrogen. 4-Bromophenylacetonitrile, 4pyridinecarboxaldehyde, DBU, 1-bromohexane, malononitrile, fluorophenylacetonitrile, methyl cyanoacetate, cyanoacetic acid, hydroxyethyl cyanoacetate, 4,4'-dimethyl-2,2'dipyridyl, benzyl bromide, 3-bromo-1-propanol and all other reagents and solvents were purchased from Aldrich and used as received. 
General Methods. ${ }^{1} \mathrm{H}$ and ${ }^{13} \mathrm{C}$ NMR spectra were recorded on a Varian $300 \mathrm{MHz}(300$ and $75 \mathrm{MHz}$ for ${ }^{1} \mathrm{H}$ and ${ }^{13} \mathrm{C}$ NMR, respectively) or a Bruker AMX $400 \mathrm{MHz}$ spectrometer using tetramethylsilane (TMS; $\delta=0 \mathrm{ppm}$ ) as an internal standard. The Fourier transform infrared (FTIR) spectra were recorded on a Perkin-Elmer1600 or a Bomen Michelson 120 FTIR spectrometer in the regions of $4000-400 \mathrm{~cm}^{-1}$. Mass spectra were measured with a Micromass Quattro LC ESI mass spectrometer (EI and TOF-ESI). The melting points were determined using a Fisher-Johns melting point apparatus. Standard differential scanning calorimetric analysis was carried out in nitrogen on a TA DSC Q100 with a heating rate of $10{ }^{\circ} \mathrm{C} \mathrm{min}^{-1}$. Thermogravimetric analysis was carried out in nitrogen on a Hi-Res TGA 2950 thermogravimetric analyzer with a heating rate of $10{ }^{\circ} \mathrm{C} \min ^{-1}$. The UV-Vis-NIR spectra were recorded on a Perkin-Elmer Lambda 900 UV-Vis-NIR spectrometer at room temperature. The solvatochromism study was conducted using organic solvents across a range of dielectric constants $(\varepsilon)$ : THF, DCM, acetone, methanol, DMF, ACN and DMSO. Due to the poor solubility of PpQDM-Met in THF, the THF solution was prepared by adding $20 \mu \mathrm{L}$ of PpQDM-Met in DMF $\left(2 \times 10^{-5}\right.$ M) into $1.98 \mathrm{~mL}$ of THF. Fluorescence emission spectra were measured on a PTI fluorescence system. The absorption and fluorescence emission spectra of all the samples were taken in $\mathrm{DMF} / \mathrm{MeOH}$ solution in a quartz cuvette with a path length of $10.0 \mathrm{~mm}$.

Single Crystal X-ray Diffraction. All diffraction measurements were carried out on a Bruker SMART APEX CCD diffractometer with graphite-monochromated Mo-K $\alpha$ ( $\lambda=$ $0.71073 \AA$ ) radiation. Data were collected using the Bruker SMART detector, processed using the SAINT Plus package from Bruker. The structures were solved by direct methods (SHELXS-97) and expanded using Fourier techniques (SHELXL-97). All 
calculations were performed using the Bruker SHELXTL97 crystallographic software package.

\section{Quantum yields measurement}

The fluorescence emission spectra were measured with a PTI spectrofluorometer. The fluorescence emission spectra of FDCNs and PpQDMs $\left(2 \times 10^{-5} \mathrm{M}\right)$ were measured at $25^{\circ} \mathrm{C}$, with excitation at $470 \mathrm{~nm}$ and $670 \mathrm{~nm}$. The fluorescent quantum yield ( $\Phi_{\mathrm{F}}$ ) of FDCNs and PpQDMs were evaluated using a relative method with reference to luminescence standards, Rhodamin 6G $\left(\Phi_{\mathrm{F}}=0.88 \text { in ethanol }\right)^{36}$ and IR-125 $\left(\Phi_{\mathrm{F}}=0.13\right.$ in DMSO), ${ }^{37}$ respectively. The quantum yield of FDCNs and PpQDMs can be expressed by eq. $3 .^{38} \Phi$ is the quantum yield (subscript " $r$ " stands for the reference and " $x$ " for the sample), $A$ is the absorbance at the excitation wavelength, $I(\lambda)$ is the relative intensity of the exciting light at wavelength $\lambda, n$ is the refractive index of the solvent for the luminescence, and $D$ is the area (on an energy scale) of the luminescence spectra. The samples and the reference were excited at the same wavelength. The sample absorbance at the excitation wavelength was kept as low as possible to avoid fluorescence errors ( $A_{\text {exc }}$ <0.1). $Q_{x}=Q_{r}\left(\frac{A_{r}\left(\lambda_{r}\right)}{A_{x}\left(\lambda_{x}\right)}\right)\left(\frac{I\left(\lambda_{r}\right)}{I\left(\lambda_{x}\right)}\right)\left(\frac{n_{x}^{2}}{n_{r}^{2}}\right)\left(\frac{D_{x}}{D_{r}}\right)$ (eq.3)

\section{Synthesis of FDCN Chromophores}

\section{2-(4-Bromophenyl)-3-pyridine-4-yl-acrylonitrile (1)}

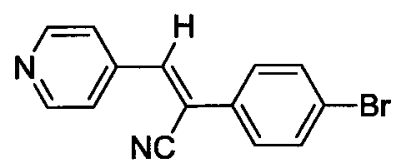


Under anhydrous and oxygen-free conditions, to a $250 \mathrm{~mL}$ round-bottomed flask, 4-bromophenylacetonitrile $(5.9 \mathrm{~g}, 30.0 \mathrm{mmol})$, 4-pyridinecarboxaldehyde $(3.0 \mathrm{~mL}, 30.0$ mmol), $80 \mathrm{~mL}$ of dry THF, and $1 \mathrm{~mL}$ of $\mathrm{DBU}$ were added. The mixture was heated to reflux for $18 \mathrm{~h}$ and then cooled to room temperature. The solvent was removed under reduced pressure; the red residue was washed with methanol $(30 \mathrm{~mL})$, filtered and dried. The final product was an off-white crystalline collected in $3.9 \mathrm{~g}$ (95\% yield); m.p:138$140{ }^{\circ} \mathrm{C} ;{ }^{1} \mathrm{HNMR}\left(300 \mathrm{MHz}, \mathrm{DMSO}-\mathrm{d}_{6}, \mathrm{TMS}, \mathrm{ppm}\right): \delta 8.75(2 \mathrm{H}, \mathrm{d}), 8.15(1 \mathrm{H}, \mathrm{s}), 7.81$ $(6 \mathrm{H}, \mathrm{m})$; IR $\left(\mathrm{KBr}, \mathrm{cm}^{-1}\right): 2217(\mathrm{C} \equiv \mathrm{N}), 1645(\mathrm{C}=\mathrm{N}), 1594,1588(\mathrm{C}=\mathrm{C})$; UV-Vis: $\lambda_{\max }=$ $312 \mathrm{~nm}(\mathrm{DMF}) ; \mathrm{MS}(\mathrm{EI}, \mathrm{m} / \mathrm{z}): 284\left[\mathrm{M}^{+}\right], 286[\mathrm{M}+2]^{+}$.

\section{2-(4-Bromophenyl)-3-pyridine-4-yl-acrylonitrile bromide salt (2)}

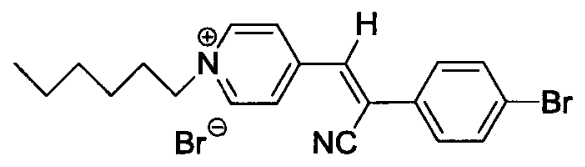

A mixture of compound $1(0.350 \mathrm{~g}, 1.23 \mathrm{mmol}), 1$-bromohexane $(2.8 \mathrm{~mL}, 20.0$ $\mathrm{mmol}$ ) and $2 \mathrm{~mL}$ of benzonitrile was refluxed for $18 \mathrm{~h}$. The reaction mixture was cooled and the precipitate was filtered. The crude product was dissolved in $10 \mathrm{~mL}$ of hot acetonitrile then added into $50 \mathrm{~mL}$ of hot ether, and the precipitate was filtered. The final product was a yellow amorphous powder collected in $0.30 \mathrm{~g}(66 \%$ yield $) ;{ }^{1} \mathrm{H}$ NMR $(300$ MHz, DMSO-d $d_{6}$, TMS, ppm): $\delta 9.22(2 \mathrm{H}, \mathrm{d}, \mathrm{J}=8.4 \mathrm{~Hz}), 8.48(2 \mathrm{H}, \mathrm{d}, \mathrm{J}=8.4 \mathrm{~Hz}), 8.44$ $(1 \mathrm{H}, \mathrm{s}), 7.86(4 \mathrm{H}, \mathrm{m}), 4.64(2 \mathrm{H}, \mathrm{t}), 1.95(\mathrm{~m}, 2 \mathrm{H}), 1.32(6 \mathrm{H}, \mathrm{m}), 0.89(3 \mathrm{H}, \mathrm{t}) ; \mathrm{IR}\left(\mathrm{KBr}, \mathrm{cm}^{-}\right.$ $\left.{ }^{1}\right): 2219(\mathrm{C} \equiv \mathrm{N}), 1637(\mathrm{C}=\mathrm{N}), 1604,1583(\mathrm{C}=\mathrm{C})$; MS (ESI, acetonitrile, $\left.\mathrm{m} / \mathrm{z}\right): 369\left(\mathrm{M}^{+}\right.$, 100); UV-Vis: $\lambda_{\max }=347 \mathrm{~nm}(\mathrm{DMF})$.

\section{Synthesis of BrDCN}




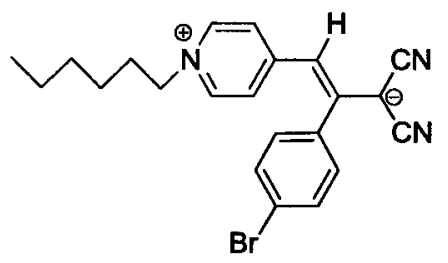

Under anhydrous and oxygen-free conditions, to a $25 \mathrm{~mL}$ round-bottomed flask, malononitrile $(0.027 \mathrm{~g}, 0.40 \mathrm{mmol})$, sodium hydride $(0.024 \mathrm{~g}, 60 \%, 0.60 \mathrm{mmol})$ and compound $2(0.1 \mathrm{~g}, 0.2 \mathrm{mmol})$ were ad ded at $0{ }^{\circ} \mathrm{C}$. After $30 \mathrm{~min}$, the insoluble inorganic salt was removed by filtration, and the filtrate solution was concentrated under reduced pressure. The residue was purified by mixture of acetone and hexane (1:1). The final product was an orange-red crystal $(0.059 \mathrm{~g}, 65 \%)$ m.p $196{ }^{\circ} \mathrm{C} ;{ }^{1} \mathrm{H}$ NMR $(400 \mathrm{MHz}$, DMSO- $\left.d_{6}\right): \delta 7.81(2 \mathrm{H}, \mathrm{d}, \mathrm{J}=7.1 \mathrm{~Hz}), 7.36(2 \mathrm{H}, \mathrm{d}, \mathrm{J}=8.7 \mathrm{~Hz}), 7.32(2 \mathrm{H}, \mathrm{d}, \mathrm{J}=8.7 \mathrm{~Hz})$, $6.34(2 \mathrm{H}, \mathrm{d}, \mathrm{J}=7.1 \mathrm{~Hz}), 5.88(1 \mathrm{H}, \mathrm{s}), 3.92(2 \mathrm{H}, \mathrm{t}), 1.22(6 \mathrm{H}, \mathrm{m}), 0.83(3 \mathrm{H}, \mathrm{t})$; IR $(\mathrm{KBr}$, $\left.\mathrm{cm}^{-1}\right): 2190\left(\mathrm{v}_{\mathrm{C} \equiv \mathrm{N}}\right.$ of cyano groups), $1640\left(\mathrm{v}_{\mathrm{C}=\mathrm{N}}\right.$ of picolinium moiety), 1571, $1450\left(\mathrm{v}_{\mathrm{C}=\mathrm{C}}\right.$ aromatic ring); MS (EI, m/z): $407[\mathrm{M}]^{+}, 409[\mathrm{M}+2]^{+}$; UV-Vis: $\lambda_{\max }=486 \mathrm{~nm}(\mathrm{DMF})$; TGA: $\mathrm{T}_{\mathrm{d}}=323^{\circ} \mathrm{C}$.

\section{2-(4-Fluorophenyl)-3-pyridine-4-yl-acrylonitrile (3)}

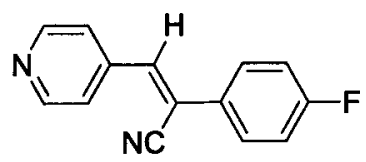

Under anhydrous and oxygen-free conditions, to a $250 \mathrm{~mL}$ round-bottomed flask 4-fluorophenylacetonitrile (4.05 g, $30.0 \mathrm{mmol})$, 4-pyridinecarboxaldehyde (3.2 g, 30.0 mmol), $90 \mathrm{~mL}$ of dry THF and $1 \mathrm{~mL}$ of DBU were added. The mixture was heated to reflux for $18 \mathrm{~h}$ and then cooled to room temperature. The solvent was removed under reduced pressure and the red residue was washed with methanol $(30 \mathrm{~mL})$, filtered and 
dried. The final product was an off-white crystalline collected in $3.4 \mathrm{~g}, 55 \%$ yield. m.p: 166-167 ${ }^{\circ} \mathrm{C} ;{ }^{1} \mathrm{H}$ NMR (400 MHz, DMSO- $\left.d_{6}\right): \delta 8.75(\mathrm{~d}, 2 \mathrm{H}), 8.15(\mathrm{~s}, 1 \mathrm{H}), 7.81(\mathrm{~m}, 6 \mathrm{H})$; IR $\left(\mathrm{KBr}, \mathrm{cm}^{-1}\right): 2216(\mathrm{C} \equiv \mathrm{N}), 1592,1543(\mathrm{C}=\mathrm{C})$; MS (EI, m/z): $224\left(\mathrm{M}^{+}, 100 \%\right)$; UV-Vis: $\lambda_{\max }=319 \mathrm{~nm}(\mathrm{DMF})$.

\section{2-(4-Fluorophenyl)-3-pyridine-4-yl-acrylonitrile bromide salt (4)}

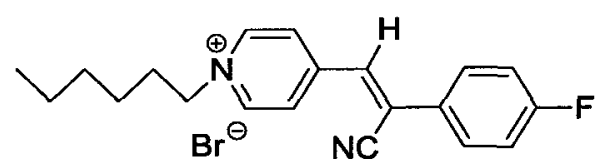

A mixture of compound $3(1.75 \mathrm{~g}, 7.80 \mathrm{mmol})$, 1-bromohexane $(20.0 \mathrm{~mL}, 143.0$ mmol) and $3 \mathrm{~mL}$ of benzonitrile was refluxed for $18 \mathrm{~h}$. The reaction mixture was cooled and the precipitate was filtered. The crude product was dissolved in $40 \mathrm{~mL}$ of hot acetonitrile then added into $250 \mathrm{~mL}$ of hot ether, the precipitated was filtered, a yellow amorphous powder collected in $2.4 \mathrm{~g}$, yield $80 \%$ yield. ${ }^{1} \mathrm{H}$ NMR (300 MHz, DMSO- $d_{6}$ ): $\delta 9.23(2 \mathrm{H}, \mathrm{d}, \mathrm{J}=8.4 \mathrm{~Hz}), 8.49(2 \mathrm{H}, \mathrm{d}, \mathrm{J}=8.4 \mathrm{~Hz}), 8.45(1 \mathrm{H}, \mathrm{s}), 7.87(4 \mathrm{H}, \mathrm{m}), 4.64(2 \mathrm{H}$, t), $1.95(\mathrm{~m}, 2 \mathrm{H}), 1.32(6 \mathrm{H}, \mathrm{m}), 0.88(3 \mathrm{H}, \mathrm{t}) ; \mathrm{IR}\left(\mathrm{KBr}, \mathrm{cm}^{-1}\right): 2224(\mathrm{C} \equiv \mathrm{N}), 1637(\mathrm{C}=\mathrm{N})$, 1597,1557 (C=C); MS (ESI, acetonitrile, m/z): $309\left(\mathrm{M}^{+}, 100\right)$; UV-Vis: $\lambda_{\max }=341 \mathrm{~nm}$ (DMF).

\section{Synthesis of FDCN}

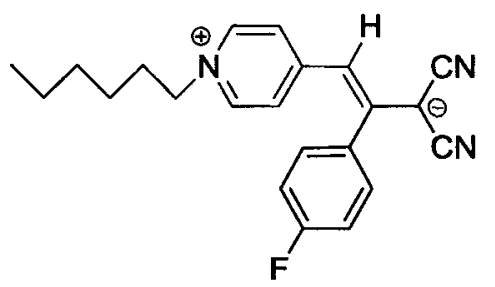

Under anhydrous and oxygen-free conditions, to a $50 \mathrm{~mL}$ round-bottomed flask malononitrile $(0.50 \mathrm{~g}, 7.4 \mathrm{mmol})$, sodium hydride $(0.30 \mathrm{~g}, 60 \%, 7.5 \mathrm{mmol})$ and 
compound $4(1.0 \mathrm{~g}, 2.6 \mathrm{mmol})$ in $20 \mathrm{~mL}$ of THF were added at $0{ }^{\circ} \mathrm{C}$. After $30 \mathrm{~min}$, the insoluble inorganic salt was removed by filtration, and the filtrate solution was concentrated under reduced pressure. The residue was purified by mixture of acetone and hexane (1:1). The final product was an orange-red crystal: $0.56 \mathrm{~g}, 63 \%$ yield; m.p: $167^{\circ} \mathrm{C}$; ${ }^{1} \mathrm{H}$ NMR (400 MHz, DMSO-d $): \delta 7.81(2 \mathrm{H}, \mathrm{d}, \mathrm{J}=7.1 \mathrm{~Hz}), 7.36(2 \mathrm{H}, \mathrm{d}, \mathrm{J}=8.7 \mathrm{~Hz}), 7.32$ $(2 \mathrm{H}, \mathrm{d}, \mathrm{J}=8.7 \mathrm{~Hz}), 6.34(2 \mathrm{H}, \mathrm{d}, \mathrm{J}=7.1 \mathrm{~Hz}), 5.88(1 \mathrm{H}, \mathrm{s}), 3.92(2 \mathrm{H}, \mathrm{t}), 1.22(6 \mathrm{H}, \mathrm{m}), 0.83$ $(3 \mathrm{H}, \mathrm{t}) ;{ }^{13} \mathrm{C}$ NMR $\left(100 \mathrm{MHz}, \mathrm{DMSO}-d_{6}\right): 161.2,151.4,139.8,133.7,130.0,129.9,119.8$, $119.2,118.2,116.6,116.4,101.7,56.7,30.4,29.8,24.9,21.8,13.7 ; \mathrm{IR}\left(\mathrm{KBr}, \mathrm{cm}^{-1}\right): 2192$ $\left(v_{C} \equiv \mathrm{N}\right.$ of cyano groups), $1640\left(v_{\mathrm{C}=\mathrm{N}}\right.$ of picolinium moiety), 1571, 1450; TOF HRMS (ESI, DMF/Acetonitrile $1: 1, \mathrm{~m} / \mathrm{z}$ ): $348.1776[\mathrm{M}+\mathrm{H}]^{+}, 370.1608[\mathrm{M}+\mathrm{Na}]^{+}$, calculated value: $347.1798[\mathrm{M}]^{+}$; UV-Vis: $\lambda_{\max }=486 \mathrm{~nm}(\mathrm{DMF}) ; \mathrm{TGA}: \mathrm{T}_{\mathrm{d}}=312{ }^{\circ} \mathrm{C} ; \quad$ Fluorescence emission $534 \mathrm{~nm}$ ( excitation at $486 \mathrm{~nm}$ ).

\section{Synthesis of FCNE}

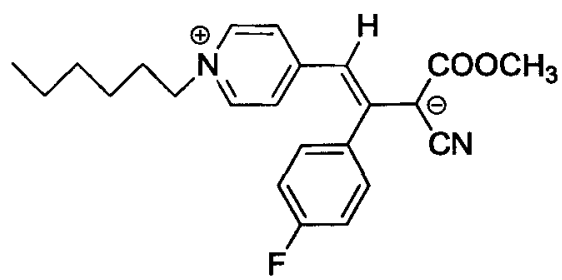

Under anhydrous and oxygen-free conditions, to a $50 \mathrm{~mL}$ round-bottomed flask methyl cyanoacetate $(0.74 \mathrm{~g}, 7.5 \mathrm{mmol})$, sodium hydride $(0.30 \mathrm{~g}, 60 \%, 7.5 \mathrm{mmol})$ and compound $4(1.0 \mathrm{~g}, 2.6 \mathrm{mmol})$ in $20 \mathrm{~mL}$ of THF were added at $0{ }^{\circ} \mathrm{C}$. The reaction was then warmed to room temperature. After $30 \mathrm{~min}$, the insoluble inorganic salt was removed by filtration, and the filtrate solution was concentrated under reduced pressure. The residue was purified by mixture of acetone and hexane $(1: 1)$. The final product was 
an orange-red crystal: $0.6 \mathrm{~g}, 61 \%$ yield. m.p: $204-206{ }^{\circ} \mathrm{C}$; ${ }^{1} \mathrm{H}$ NMR $(400 \mathrm{MHz}$, DMSO$\left.d_{6}\right): \delta 7.72(2 \mathrm{H}, \mathrm{d}, \mathrm{J}=6.7 \mathrm{~Hz}), 7.29(2 \mathrm{H}, \mathrm{d}, \mathrm{J}=8.5 \mathrm{~Hz}), 7.21(2 \mathrm{H}, \mathrm{d}, \mathrm{J}=8.5 \mathrm{~Hz}), 7.20$ $(1 \mathrm{H}, \mathrm{s}), 6.16(2 \mathrm{H}, \mathrm{d}, \mathrm{J}=6.7 \mathrm{~Hz}), 3.88(2 \mathrm{H}, \mathrm{t}), 3.57(3 \mathrm{H}, \mathrm{s}), 1.65(6 \mathrm{H}, \mathrm{t}), 1.21(6 \mathrm{H}, \mathrm{m})$, $0.83(3 \mathrm{H}, \mathrm{t}) ;{ }^{13} \mathrm{C}$ NMR $\left(100 \mathrm{MHz}, \mathrm{DMSO}-d_{6}\right): 163.2,160.7,152.2,139.3,136.7,129.9$, $122.2,118.6,116.3,116.1,104.7,75.1,56.549 .9,30.4,29.8,24.9,21.7,13.7$; IR $(\mathrm{KBr}$, $\left.\mathrm{cm}^{-1}\right): 2180(\mathrm{C} \equiv \mathrm{N}), 1750(\mathrm{C}=\mathrm{O}), 1639,1505 ; \mathrm{MS}(\mathrm{EI}, \mathrm{m} / \mathrm{z}): 380\left(\mathrm{M}^{+}, 100 \%\right)$; TOF HRMS (ESI, Acetonitrile, m/z): $381.1826[\mathrm{M}+\mathrm{H}]{ }^{+}, 403.1637[\mathrm{M}+\mathrm{Na}]^{+}$, calculated value: $380.1900[\mathrm{M}]^{+}$.

\section{Synthesis of FCNBen}

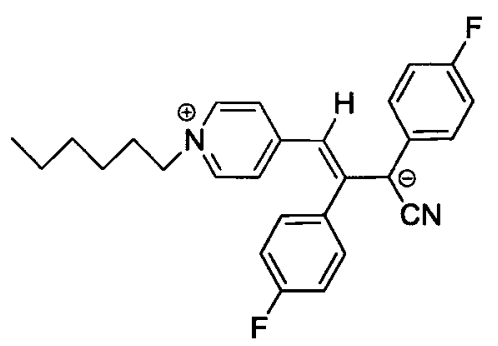

Under anhydrous and oxygen-free conditions, to a $50 \mathrm{~mL}$ round-bottomed flask, 4-fluorophenylacetonitrile $(0.2 \mathrm{~g}, 0.5 \mathrm{mmol})$, sodium hydride $(0.060 \mathrm{~g}, 60 \%, 1.5 \mathrm{mmol})$ and compound $2(0.20 \mathrm{~g}, 0.44 \mathrm{mmol})$ in $5 \mathrm{~mL}$ of THF were added at $0{ }^{\circ} \mathrm{C}$. Then the reaction was warmed to room temperature. After $30 \mathrm{~min}$, the insoluble inorganic salt was removed by filtration, and the filtrate solution was concentrated under reduced pressure. The residue was purified by mixture of acetone and hexane (1:1). The final product was an orange-red crystal with $61 \%$ yield. m.p: $126-128^{\circ} \mathrm{C}$; IR $\left(\mathrm{KBr}, \mathrm{cm}^{-1}\right): 2179(\mathrm{C} \equiv \mathrm{N})$, 1644, 1504; MS (EI, m/z): $416\left(\mathrm{M}^{+}, 100 \%\right)$; UV-Vis: $\lambda_{\max }=484 \mathrm{~nm}(\mathrm{DMF})$.

\section{2-Hydroxyethyl cyanoacetate}

A mixture of $4.25 \mathrm{~g}(50.0 \mathrm{mmol})$ of cyanoacetic acid, $3.41 \mathrm{~g}(55.0 \mathrm{mmol})$ of ethylene glycol, and $0.2 \mathrm{~g}$ of $\mathrm{TsOH}$ in $15 \mathrm{~mL}$ of benzene was heated under reflux in a 
round-bottom flask equipped with a Dean-Stark trap. After the calculated volume of water $(0.9 \mathrm{~mL})$ was distilled off, the solvent was evaporated then fractionation of the residue by vacuum distillation under nitrogen gave $2.58 \mathrm{~g}(40 \%)$ of 2-hydroxyethyl cyanoacetate. ${ }^{1} \mathrm{H}$ NMR (300 MHz, DMSO- $\left.d_{6}\right): \delta$ (TMS, ppm) $3.52\left(\mathrm{~s}, 2 \mathrm{H}, \mathrm{CH}_{2} \mathrm{CN}\right), 4.02$ (m, 2H, $\left.\mathrm{CH}_{2} \mathrm{OH}\right), 4.4(\mathrm{~s}, 1 \mathrm{H}, \mathrm{OH}), 4.55\left(\mathrm{~m}, 2 \mathrm{H}, \mathrm{COOCH}_{2}\right)$; IR $\left(\mathrm{NaCl}, \mathrm{cm}^{-1}\right): 3426(\mathrm{OH})$, $2268(\mathrm{C} \equiv \mathrm{N}), 1743(\mathrm{C}=\mathrm{O}), 1194$.

\section{Synthesis of FCNOH}

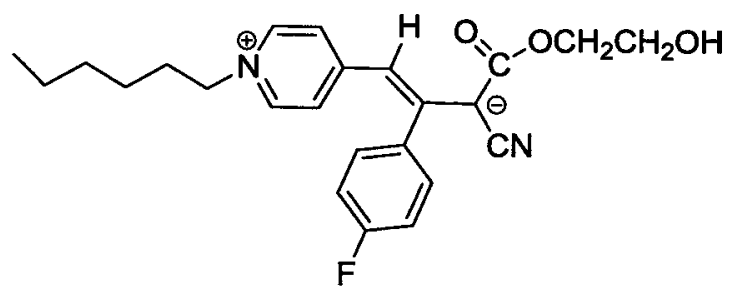

Under anhydrous and oxygen-free conditions, to a $50 \mathrm{~mL}$ round-bottomed flask, 2-hydroxyethyl cyanoacetate $(0.99 \mathrm{~g}, 7.7 \mathrm{mmol})$, sodium hydride $(0.30 \mathrm{~g}, 60 \%, 7.5 \mathrm{mmol})$ and compound $4(1.0 \mathrm{~g}, 2.6 \mathrm{mmol})$ in $20 \mathrm{~mL}$ of THF were added at $0{ }^{\circ} \mathrm{C}$. Then the reaction was warmed to room temperature. After $60 \mathrm{~min}$, the insoluble inorganic salt was removed by filtration, and the filtrate solution was concentrated under reduced pressure. The residue was purified by mixture of acetone and hexane (1:1). The final product was a dark-red solid: $0.42 \mathrm{~g}, 40 \%$ yield. IR $\left(\mathrm{KBr}, \mathrm{cm}^{-1}\right): 3387(\mathrm{OH}), 2934,2175(\mathrm{C} \equiv \mathrm{N}), 1637$ $(\mathrm{C}=\mathrm{O}), 1509 ;$ MS (EI, m/z): $410\left(\mathrm{M}^{+}, 100 \%\right)$; UV-Vis: $\lambda_{\max }=496 \mathrm{~nm}(\mathrm{DMF}) \cdot \lambda_{\max }=556$ $\mathrm{nm}\left(\mathrm{CHCl}_{3}\right)$. Negative solvatochromism of $60 \mathrm{~nm}$ was observed from $\mathrm{CHCl}_{3}$ to DMF.

\section{Synthesis of PpQDMs}

\section{1,4,4'-Trimethyl-2, 2'-dipyridylium iodide}




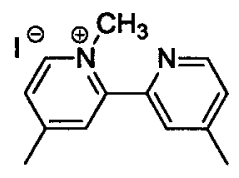

4, 4'-Dimethyl-2, 2'-dipyridyl (1.1 $\mathrm{g}, 6.0 \mathrm{mmol})$ was dissolved in $5 \mathrm{~mL}$ of methyl iodide. The mixture was refluxed at $40{ }^{\circ} \mathrm{C}$ for $20 \mathrm{~h}$. The excess methyl iodide was removed by rotary evaporation. The solid residual was washed with acetone and hexane. An off-white powder was obtained: $1.85 \mathrm{~g}, 95 \%$. melting point $201-202{ }^{\circ} \mathrm{C}$. ${ }^{1} \mathrm{H}$ NMR (300 MHz, DMSO-d 6 ,TMS, ppm): $\delta 9.02(1 \mathrm{H}, \mathrm{d}, J=6.2 \mathrm{~Hz}), 8.71(1 \mathrm{H}, \mathrm{d}, J=3.2 \mathrm{~Hz})$, $8.16(1 \mathrm{H}, \mathrm{s}), 8.07(1 \mathrm{H}, \mathrm{d}, J=6.2 \mathrm{~Hz}), 7.82(1 \mathrm{H}, \mathrm{s}), 7.56(1 \mathrm{H}, \mathrm{d}, J=3.2 \mathrm{~Hz}), 4.21(3 \mathrm{H}, \mathrm{s})$, $2.68(3 \mathrm{H}, \mathrm{s}), 2.52(3 \mathrm{H}, \mathrm{s})$; MS (ESI, MeOH, m/z) (\%): $199.3\left(\mathrm{M}^{+}, 100 \%\right)$.

\section{1-Benzyl -4, 4'-dimethyl-2, 2'-dipyridylium bromide}

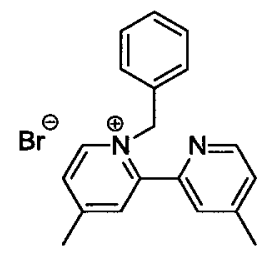

A similar procedure for the synthesis of 1,4,4'-trimethyl-2,2'-dipyridylium bromide: A mixture of 4, 4'-dimethyl-2, 2'-dipyridyl ( $8.832 \mathrm{~g}, 48.0 \mathrm{mmol})$ and benzyl bromide $(5.7 \mathrm{~mL}, 48 \mathrm{mmol})$ were refluxed for $24 \mathrm{~h}$ and then cooled to room temperature. Then the cooled viscous mixture was slowly added into ether, and an off-yellow fine powder was precipitated out. The precipitate was filtered and dried in a vacuum oven .Yield $1.7 \mathrm{~g}(90 \%)$ with a melting point of $158{ }^{\circ} \mathrm{C} .{ }^{1} \mathrm{H}$ NMR $\left(300 \mathrm{MHz}, \mathrm{CDCl}_{3}\right.$, TMS, ppm): $\delta 9.66(1 \mathrm{H}, \mathrm{d}, J=6.2 \mathrm{~Hz}), 8.61(1 \mathrm{H}, \mathrm{d}, J=6.2 \mathrm{~Hz}), 8.16(1 \mathrm{H}, \mathrm{s}), 8.07(1 \mathrm{H}$, $\mathrm{d}, J=6.2 \mathrm{~Hz}), 7.82(1 \mathrm{H}, \mathrm{s}), 7.56(1 \mathrm{H}, \mathrm{d}, J=3.2 \mathrm{~Hz}), 6.17(2 \mathrm{H}, \mathrm{s}), 2.70(3 \mathrm{H}, \mathrm{s}), 2.42(3 \mathrm{H}$, s); MS (ESI, MeOH, m/z) (\%): $275.4\left(\mathrm{M}^{+}, 100 \%\right)$. 


\section{1-Hydroxypropyl -4, 4'-dimethyl-2, 2'-dipyridylium bromide}

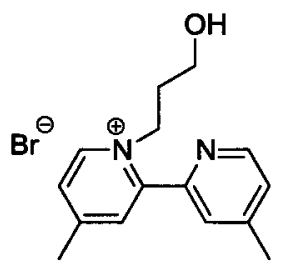

A similar procedure for the synthesis of 1-hydroxypropyl-4,4'-dimethyl-2,2'dipyridylium bromide: 4,4'-dimethyl-2, 2'-dipyridyl (3.0 g, $16 \mathrm{mmol})$ was dissolved in $15 \mathrm{~mL}$ of 3-bromo-1-propanol. The mixture reacted at $55-60^{\circ} \mathrm{C}$ for $72 \mathrm{~h}$ and then cooled to room temperature. Then the cooled viscous mixture was slowly added into ether, offwhite fine powder was precipitated out. The precipitate was filtered and dried in vacuum oven. Yield $4.13 \mathrm{~g}(80 \%) .{ }^{1} \mathrm{H} \mathrm{NMR}\left(300 \mathrm{MHz} \mathrm{CDCl}_{3}\right): \delta 8.71(1 \mathrm{H}, \mathrm{d}, J=6.4 \mathrm{~Hz}), 8.52(1$ $\mathrm{H}, \mathrm{d}, J=5.1 \mathrm{~Hz}), 7.88(1 \mathrm{H}, \mathrm{d}, J=6.4 \mathrm{~Hz}), 7.84(1 \mathrm{H}, \mathrm{s}), 7.57(1 \mathrm{H}, \mathrm{s}), 7.48(1 \mathrm{H}, \mathrm{d}, J=5.1$ $\mathrm{Hz}), 4.55(2 \mathrm{H}, \mathrm{t}, J=7.5 \mathrm{~Hz}), 3.39(2 \mathrm{H}, \mathrm{t}, J=5.9 \mathrm{~Hz}), 2.61(3 \mathrm{H}, \mathrm{s}), 2.43(3 \mathrm{H}, \mathrm{s}), 1.87(2 \mathrm{H}$, m); MS (ESI, MeOH, m/z) (\%): $243.4\left(\mathrm{M}^{+}, 100 \%\right)$.

\section{Synthesis of the PpQDM-Met}

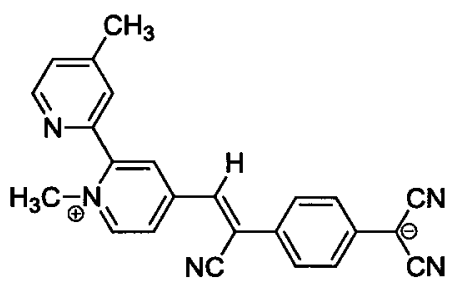

To a $30 \mathrm{~mL}$ methanol solution containing 1,4,4'-trimethyl-2,2'-dipyridylium iodide (3.3 g, $10 \mathrm{mmol}), 200 \mathrm{~mL}$ of methanol solution of Li-TCNQ (4.22 $\mathrm{g}, 20.0 \mathrm{mmol}$ ) was added and the resulting green colored mixture was refluxed for $1 \mathrm{~h} .5 \mathrm{~mL}$ of a methanol solution of 1-piperidineethanol $(1.3 \mathrm{~g}, 10 \mathrm{mmol})$ was then added slowly over the course of $1 \mathrm{~h}$. The reaction was monitored using the UV-Vis spectra of the reaction 
mixture in DMF and typically the reaction can be finished within $48 \mathrm{~h}$. Therefore, an aliquot of the reaction mixture was taken and dissolved in DMF for a UV-Vis measurement. The reaction was stopped until the CT band of the chromophore $(\sim 680 \mathrm{~nm})$ was much stronger than the characteristic peaks of the radical anion $(\sim 420,747$ and 848 $\mathrm{nm})$. The solution gradually turned to a blue color. After the reaction mixture was cooled to room temperature, the green precipitate was filtered and washed with methanol and ethyl ether. The final product was a green powder collected in $60 \%(2.28 \mathrm{~g}) .{ }^{1} \mathrm{H}$ NMR $\left(400 \mathrm{MHz}, \mathrm{DMSO}-\mathrm{d}_{6}\right): \delta 9.01(1 \mathrm{H}, \mathrm{d}, J=6.6 \mathrm{~Hz}), 8.72(1 \mathrm{H}, \mathrm{d}, J=5.5), 8.39(2 \mathrm{H}, \mathrm{d}, J=$ $6.6 \mathrm{~Hz}), 8.38(1 \mathrm{H}, \mathrm{s}), 7.88(1 \mathrm{H}, \mathrm{s}), 7.80(1 \mathrm{H}, \mathrm{s}), 7.58(2 \mathrm{H}, \mathrm{d}, J=8.7 \mathrm{~Hz}), 7.56(1 \mathrm{H}, \mathrm{d}, J=$ $5.5 \mathrm{~Hz}), 6.87(2 \mathrm{H}, \mathrm{d}, J=8.7 \mathrm{~Hz}), 4.19(3 \mathrm{H}, \mathrm{s}), 2.48(3 \mathrm{H}, \mathrm{s}) ;{ }^{13} \mathrm{C}$ NMR $(75 \mathrm{MHz}, \mathrm{DMSO}-$ $\left.\mathrm{d}_{6}\right): \delta 152.28,150.45,150.00,149.73,147.78,147.55,128.16,127.98,127.13,126.95$, $125.59,123.80,123.47,121.59,120.36,119.02,118.79,117.32,46.80,21.12$; TOF HRMS (ESI, DMF/Acetonitrile 1:1, m/z) (\%): $376.1615[\mathrm{M}+\mathrm{H}]^{+}, 396.1422[\mathrm{M}+\mathrm{Na}]^{+}$, calculated value: $375.1484[\mathrm{M}]^{+}$; IR $\left(\mathrm{KBr}, \mathrm{cm}^{-1}\right): 2226,2173$ and $2137(\mathrm{C} \equiv \mathrm{N}), 1629$, $1600(\mathrm{C}=\mathrm{C}) ; \mathrm{UV}-\mathrm{Vis}(\mathrm{DMF}): \lambda_{\max }(\mathrm{nm})=680 \mathrm{~nm}$

\section{Synthesis of PpQDM-Ben}

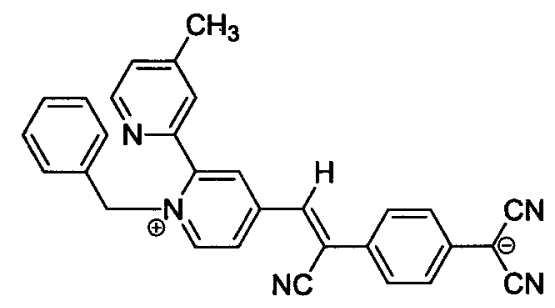

A similar procedure as PpQDM-Met: 1-benzyl-4,4'-dimethyl-2,2'-dipyridylium; bromide $(3.55 \mathrm{~g}, 10.0 \mathrm{mmol})$ was used to afford $1.8 \mathrm{~g}$ of product ( $40 \%$ yield). ${ }^{1} \mathrm{H} \mathrm{NMR}$ $\left(300 \mathrm{MHz}, \mathrm{DMSO}-\mathrm{d}_{6}\right): \delta 9.15(1 \mathrm{H}, \mathrm{d}, J=7.1), 8.68(1 \mathrm{H}, \mathrm{d}, J=4.7), 8.46(1 \mathrm{H}, \mathrm{d}, J=7.1$ 
Hz), $8.36(1 \mathrm{H}, \mathrm{s}), 7.87(1 \mathrm{H}, \mathrm{s}), 7.59(2 \mathrm{H}, \mathrm{d}, J=8.7 \mathrm{~Hz}), 7.51(1 \mathrm{H}, \mathrm{d}, J=4.7 \mathrm{~Hz}), 7.28$ $(3 \mathrm{H}, \mathrm{m}), 7.01(2 \mathrm{H}, \mathrm{m}), 6.87(2 \mathrm{H}, \mathrm{d}, J=8.7 \mathrm{~Hz}), 5.94(2 \mathrm{H}, \mathrm{s}), 2.37(3 \mathrm{H}, \mathrm{s}) ;{ }^{13} \mathrm{C}$ NMR $(75$ MHz, DMSO-d $\left.\mathrm{d}_{6}\right): \delta 150.63,150.40,149.32,147.54,147.34,146.58,128.70,128.61$, $127.91,127.72,126.53,126.51,126.48,125.12,124.64,124.44,123.64,123.55,120.86$, 118.71, 118.57, 117.33, 59.90, 20.44; TOF HRMS (ESI, DMF/Acetonitrile 1:1, m/z): $452.1701[\mathrm{M}+\mathrm{H}]^{+}$, calculated value: $451.1797[\mathrm{M}]^{+}$; IR $\left(\mathrm{KBr}, \mathrm{cm}^{-1}\right): 2214,2176$ and $2138(\mathrm{C} \equiv \mathrm{N}), 1625,1600(\mathrm{C}=\mathrm{C})$.

\section{Synthesis of PpQDM-C3OH}

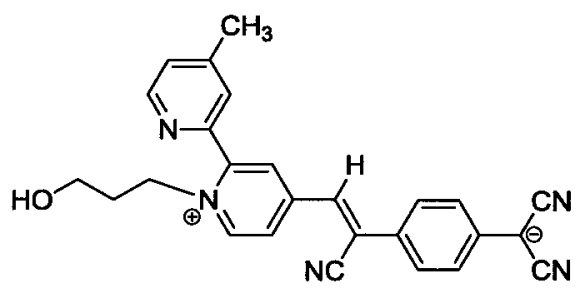

A similar procedure as PpQDM-Met: 1-hydroxypropyl-4,4'-dimethyl-2,2'dipyridylium bromide $(0.13 \mathrm{~g}, 0.40 \mathrm{mmol})$ was used to afford $0.067 \mathrm{~g}$ of product $(40 \%$ yield). m.p. $248^{\circ} \mathrm{C}$ from DSC. ${ }^{1} \mathrm{H}$ NMR $\left(300 \mathrm{MHz}, \mathrm{DMSO}-\mathrm{d}_{6}\right): \delta 9.06(1 \mathrm{H}, \mathrm{d}, J=6.8$ $\mathrm{Hz}), 8.70(1 \mathrm{H}, \mathrm{d}, J=5.0 \mathrm{~Hz}), 8.41(1 \mathrm{H}, \mathrm{d}, J=6.8 \mathrm{~Hz}), 8.34(1 \mathrm{H}, \mathrm{d}, J=2.1 \mathrm{~Hz}), 7.80(1 \mathrm{H}$, s), $7.87(1 \mathrm{H}, \mathrm{s}), 7.59(2 \mathrm{H}, \mathrm{d}, J=8.8 \mathrm{~Hz}), 7.56(1 \mathrm{H}, \mathrm{d}, J=5.0 \mathrm{~Hz}), 6.88(1 \mathrm{H}, \mathrm{d}, J=8.8$ $\mathrm{Hz}), 4.64(2 \mathrm{H}, \mathrm{m}), 4.62(1 \mathrm{H}, \mathrm{s}), 3.36(2 \mathrm{H}, \mathrm{m}), 2.49(3 \mathrm{H}, \mathrm{s}), 1.88(2 \mathrm{H}, \mathrm{m}) ;{ }^{13} \mathrm{C}$ NMR $(75$ $\left.\mathrm{MHz}, \mathrm{CDCl}_{3}\right): \delta 151.14,149.68,149.38,149.03,146.97,145.94,127.73,127.10,126.10$, $126.03,124.39,122.98,122.45,120.72,119.53,118.11,116.41,56.88,55.02,32.52$, 20.20; IR $\left(\mathrm{KBr}, \mathrm{cm}^{-1}\right): 2218,2175$ and $2137(\mathrm{C} \equiv \mathrm{N}), 1625,1600(\mathrm{C}=\mathrm{C})$; TOF HRMS (ESI, DMF/Acetonitrile 1:1, m/z) (\%): $420.1869[\mathrm{M}+\mathrm{H}]^{+}, 442.1614[\mathrm{M}+\mathrm{Na}]^{+}$, calculated value: $419.1746[\mathrm{M}]^{+}$. 


\subsection{References}

(1) (a)Gorman, C. B.; Marder, S. R. Proc. Natl. Acad. Sci. USA 1993, 90, 11297. (b)Robin, B. H.; Dalton, L. R.; Harper, A. W.; Wang, F.; Zhang, C.; Todorova, G.; Lee, M.; Aniszfeld, R.; Garner, S.; Steier, W. H.; Houbrecht, S.; Persoons, A.; Ledoux, I.; Zyss, J.; Jen, A. K.-Y. Chem Phys. 1999, 245, 35. (c)Zhang, C.; Dalton, L. R.; Oh, M.; Zhang, H.; Steier, W. H. Chem Mater. 2001, 13, 3043.

(2) (a)Metzger, R. M.; Heimer, N. E.; Aswell, G. J. Mol. Cryst. Liq. Cryst. 1984, 107, 133. (b)Kay, A. J.; Woolhouse, A. D.; A. D.; Zhao, Y.; Clays, K.; J. Mater. Chem. 2004, 14, 1321.

(3) Ashwell, G. J. Organic Materials for Nonlinear Optics; Ashwell, G. J.; Bloor, D. Eds; Royal Soc. Chem.: Cambridge, 1993

(4) Metzger, R. M.; Heimer, N. E.; Aswell, G. J. Mol. Cryst. Liq. Cryst. 1984, 107, 133.

(5) Ashwell, G. J. Thin Solid films 1990, 186, 155.

(6) Kay, A. J.; Woolhouse, A. D.; A. D.; Clays, K.; J. Mater. Chem. 2004, 14, 1321.

(7) Metzger, R. M.; Ulf Hopfner, B. C.; Lakshmikanthanm, M. V.; Vuillaume, D.; Kawai, T.; Wu, X.; Tachibana, H.; Hughes, T. V.; Sakurai, H.; Baldwin, J. W.; Hosch, C.; Cava, M. P.; Brehmer, L.; Ashwell, G. J. J. Am. Chem. Soc. 1997, 119, 10455.

(8) Ashwell, G. J. Eur. Patent EP 0391631 A1, 1990.

(9) Weir, C. A. M.; Hadizad, T.; Beaudin, A. M. R.; Wang, Z. Y. Tetrahedron Lett. 2003, 44, 4697 .

(10) Beaudin, A. M. R.; Song, N. H.; Bai, Y. W.; Men, L.; Gao, J. P.; Wang, Z. Y.; Szablewski, M; Cross, G.; Wenseleers, W. Campo, J.; Goovaerts, E. Chem. Mater. 2006, 18, 1079.

(11) (a)Wurther, F.; Yao, S. Angew. Chem. Int. Ed. 2000, 39, 1978. (b)Wurther, F.; Yao, S.; Debaerdemaeker, T.; Wortmann, R. J. Am. Chem. Soc. 2002, 124, 9431.

(12) Xiong, Y.; Tang, H.; Zhang, J.; Wang, Z. Y.; Campo, J.; Wenseleers, W.; Goovaerts, E. Chem. Mater. 2008, $20,7465$. 
(13) Guseva, T. I.; Senchenya, N. G.; Golding, I. P.; Mager, K. A.; Gololobov, Y. G. Russ. Chem. Bull. 1993, 42, 478.

(14) Wu, Y.-L.; Bures, F.; Jarowski, P. D.; Schweizer, W. B.; Boudon, C.; Gisselbrecht, J.-P.; Diederich, F. Chem. Eur. J. 2010, 16, 9592.

(15) (a)Andreu, R.; Blesa, M. J.; Carrasquer, L.; Garin, J.; Orduna, J.; Villacampa, B.; Alcala, R.; Casado, J.; Delgado, M. C. R.; Navarrete, J. T. L.; Allain, M. J. Am. Chem. Soc. 2005, 127, 8835. (b)Kawase, T.; Wakabayashi, M.; Takahashi, C.; Oda, M. Chem. Lett. 1997, 1055.

(16) Sutton, L. E. Tables of Interatomic Distances and Configuration in Molecules and Ions, Special Publication No. 18; Mitchell, A. D.; Somerfield, A. E.; Cross, L. C. Eds. The Chemical Society: London, 1965.

(17) Allen, F. H.; Kennard, O.; Watson, D. G.; Brammer, L.; Orpen, A. G.; Taylor, R. J. Chem. Soc., Perkin Trans. 1987, 2, S1.

(18) De Ridder, D. J. A.; Heijdenrijk, D.; Schenk, H.; Dommisse, R. A.; Lemiere, G. L.; Lepoivre, J. A.; Alderweireldt, F. A. Acta Crystallogr. 1990, C46, 2197.

(19) Cole, J. C.; Cole, J. M.; Cross, G. H.; Farsari, M.; Howard, J. A. K.; Szablewski, M. Acta Cryst. 1997, B53, 812.

(20) Wurthner, F.; Yao, S.; Debaerdemaeker, T.; Wortmann, R. J. Am. Chem. Soc. 2002, 124, 9431 .

(21) (a)Wurthner, F.; Sens, R.; Etzbach, K.-H.; Seybold, G. Angew. Chem., Int. Ed. 1999, 38, 1649. (b)Wurthner, F. Synthesis 1999, 2103. (c)Wurthner, F.; Yao, S.; Wortmann, R. J. Inf. Rec. 2000, 25, 69. (d)Wurthner, F.; Yao, S.; Schilling, J.; Wortmann, R.; Redi-Abshiro, M.; Mecher, E.; Gallego-Gomez, F.; Meerholz, K. J. Am. Chem. Soc. 2001, 123, 2810. (e)Mecher, E.; Gallego-Gomez, F.; Bra“uchle, C.; Meerholz, K.; Wortmann, R.; Yao, S.; Sautter, A.; Wurthner, F. Proc. SPIE 2000, 4104, 118.

(22) Melby, L. R.; Harder, R. J.; Hertler, W. R.; Mahler, W.; Benson, R. E.; Mochel, W. E. J. Am. Chem. Soc. 1962, 84, 3374. 
(23) (a)Albert, I. D. L.; Marks, T. J.; Ratner, M. A. J. Am. Chem. Soc. 1998, 120, 11174. (b)Albert, I. D. L.; Marks, T. J.; Ratner, M. A. J. Am. Chem. Soc. 1997, $119,3155$.

(24) Staneva, D.; Grabchev, I.; Soumillion, J. P.; Bojinov, V. J. Photochem. Photobiol. A. Chem. 2007, 189, 192.

(25) Reichardt, C. Chem. Rev. 1994, 94, 2319.

(26) Szablewski, M.; Thomas, P. R.; Thornton, A.; Bloor, D.; Cross, G. H.; Cole, J. M.; Howard, J. A. K.; Malagoli, M.; Meyer, F.; Bredas, J.; Wenseleers, W,; Goovaerts, E.; J. Am. Chem. Soc. 1997, 119, 3144.

(27) (a)Lu, L.; Lachicotte, R. J.; Penner, T. L.; Whitten, D. G. J. Am. Chem. Soc. 1999, 121, 8146. (b) Meech, S. R.; Phillips, D. Chem. Phys. 1983, 80, 317.

(28) Ying Xiong "Development of Zwitterinic Chromophores for Electro-Optic Applications", Ph. D. Thesis, Carleton University 2008.

(29) Mes, G. F.; Jong, B.; Ramesdonk, H. J.; Verhoeven, J. W.; Warman, J. M.; Haas, M. P.; Horsman-van den Dool, L. E. W. J. Am. Chem. Soc. 1984, 106, 6524.

(30) (a)Weber, G.; Farris, F. J. Biochemistry 1979, 18, 3075. (b)Mes, G. F.; Jong, B.; Ramesdonk, H. J.; Verhoeven, J. W.; Warman, J. M.; Haas, M. P.; Horsmanvanden Dool, L. E. W. J. Am. Chem. Soc.1984, 106, 6524.

(31) Kawski, A; Bilot, L. Acta Phys. Pol. 1964, 26, 41.

(32) Kay, A. J.; Woolhouse, A. D.; Zhao, Y.; Clays, K.; J. Mater. Chem. 2004, 14, 1321.

(33) Szablewski, M.; Thomas, P.; Thornton, A.; Bloor, D.; Cross, G. H.; Cole, J.; Howard, J.; Malagoli, M.; Meyers, F.; Bredas, J.; Wenseleers, W.; Goovaerts, E. J. Am. Chem. Soc. 1997, 119, 3144.

(34) Cross, G. H.; Hackman, N. A.; Thomas, P. R.; Szablewski, M.; Palsson, L. O.; Bloor, D. Opt. Mater. 2003, 21, 29.

(35) Oudar, J. L.; Chemla, D. S. Chem. Phys. 1977, 66, 2664.

(36) Olmsted, J. J. Phys. Chem. 1979, 83, 2581.

(37) Benson, R. C.; Kues, H. A. J. Chem. Eng. Data 1977, 22, 379.

(38) Demas, J. N.; Crosby, G. A. J. Phys. Chem. 1971, 75, 991. 


\section{Chapter 3 Synthesis of Zwitterionic Nonlinear Optical Polymers}

\subsection{Introduction}

As discussed in Chapter 1, for EO applications, NLO chromophores are usually doped into or covalently bonded onto polymers, followed by poling under an externally applied electric field at the glass transition temperature $\left(T_{g}\right)$ of the materials in order to achieve a noncentrosymmetric order. Therefore it is of importance to develop suitable host polymers that can impart efficiently the large hyperpolarizability of the NLO chromophores to a large and stable macroscopic EO activity. Grafting NLO chromophores onto polymer chains can effectively prevent the polar chromophores from forming aggregates while eliminating any possible phase separation.

In general, development of host polymers for highly polar zwitterionic chromophores needs to meet several critical requirements for device applications, including 1) the host polymer should be structurally compatible to form covalent bonds with the chromophore under proper reaction conditions; 2) good polarity compatibility with zwitterionic chromophores, the host polymer should be physically able to provide a desired polar or nonpolar medium; 3) high temporal stability to stabilize the oriented chromophores, which can be achieved by high $\mathrm{T}_{\mathrm{g}}$ polymers or cross-linked organic networks; 4) optical transparency at telecommunication wavelengths. The host polymers should have a low content of $\mathrm{C}-\mathrm{H}, \mathrm{N}-\mathrm{H}$ and $\mathrm{O}-\mathrm{H}$ bonds. ${ }^{1}$ These bonds are known to have overtone absorptions near the telecommunication wavelengths, which causes optical loss.

To choose a suitable host polymer for FDCN and PpQDM, these chromophores were first physically doped into various commercial available polymers to form guesthost systems for film-forming and compatibility tests. Then NLO chromophore grafted 
polymers were prepared. To avoid the degradation of the fragile chromophores during the classical polymerization process for synthesis of zwitterionic NLO polymers, an approach of post-functionalization was employed. ${ }^{2}$ First, the starting polymer is prepared. Then, NLO chromophores are attached to the polymer under a mild reaction condition in high yields with a minimum release of by-products. This strategy allows for optimization of the desired property, such as EO coefficients or thermal stability, through the large variety of different materials and synthetic conditions.

This chapter describes the design, synthesis and characterization of host polymers for FDCN and PpQDM chromophores, and the synthesis of FDCN-based NLO polymers and PpQDM-based crosslinkable NLO polyimides. EO properties of these two series of NLO polymers will be introduced in Chapter 4.

\subsection{Synthesis and Characterization of FDCN-based NLO Polymers}

\subsubsection{Synthesis of Cyanoacetate Containing MMA Host Polymers (A1)}

A wide variety of polymer matrices have been exploited for development of EO materials and devices, such as poly(methyl methacrylate) (PMMA), ${ }^{3}$ polycarbonate, ${ }^{4}$ polyurethane, ${ }^{5}$ polyimide, ${ }^{6}$ polyethersulfone (PES), ${ }^{7}$ sol-gel, ${ }^{8}$ and polyvinylphenol, ${ }^{9}$ etc. Among guest-host EO polymer systems, doping inorganic or NLO chromophores, such as disperse red one (DR1) into PMMA-based polymer materials have been widely studied $^{10}$, because of the flexibility and high transparency of the polymer, combined with the high thermal stability of organic chromophores; doping zwitterionic chromophores into PES have been extensively studied in our group ${ }^{6 \mathrm{~b}, 6 \mathrm{c}, 7 \mathrm{~b}, 7 \mathrm{c}, 7 \mathrm{e}}$ and most of the doped PES films exhibited higher poling efficiency than those in other polymer matrices for PQDM and PeQDM chromophores. 
Based on the preliminary study, PMMA and PES were selected as the host polymers for FDCN doping, due to their availability, processibility, and background knowledge. A relatively large amount of FDCN (5-10 wt \%) was blended into PMMA and PES forming a red color film, no phase separation was observed. Functional PMMA was then designed and synthesized for grafting FDCN chromophore, PES was used to make FDCN-PES guest-host polymer films for EO coefficient measurement for comparison (In Chapter 4).

Therefore, a series of cyanoacetate-containing MMA copolymers A1 were designed (Scheme 3.1) as host polymers for FDCN chromophores. On the other hand, the cyanoacetate functionality in the MMA polymer offers a reactive site for the simple attachment of FDCN chromophores, which will be shown in the following section.

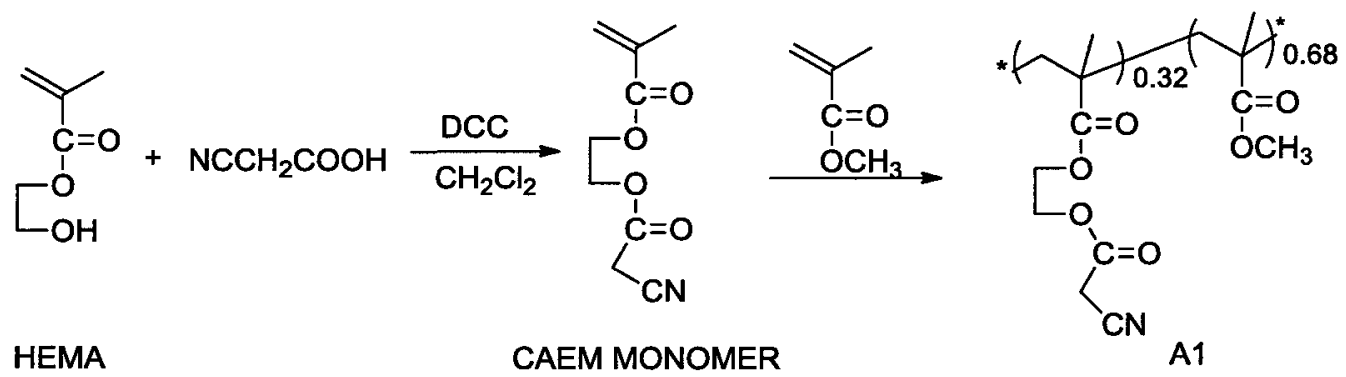

Scheme 3.1. Synthesis of CAEM and A1 polymers.

First, the appropriate monomer, 2-cyanoacetoxyethyl methacrylate (CAEM) was prepared by esterification of 2-hydroxyethyl methacrylate (HEMA) with cyanoacetic acid using dicyclohexylcarbodiimide (DCC) as the coupling agent. ${ }^{11}$

Free radical copolymerization of CAEM with various amounts of MMA led to CAEM-MMA based copolymers A1. As an example, A1-1 was synthesized by the copolymerization reaction of CAEM and MMA in dry toluene at $65-70{ }^{\circ} \mathrm{C}$ yielding a white powder in a low molecular weight (inherent viscosity of $0.22 \mathrm{dL} / \mathrm{g}$ ), which has a $\mathrm{T}_{\mathrm{g}}$ 
of $83{ }^{\circ} \mathrm{C}$ and good thermal stability $\left(220^{\circ} \mathrm{C}\right.$ for $5 \%$ weight loss) in nitrogen (Appendix B, Figures S3.1 and S3.2).

The ${ }^{1} \mathrm{H}$ NMR spectrum of A1-1 demonstrated that 32 mol\% of CAEM was incorporated into A1-1 (Figure 3.1). This is in agreement with the composition expected from the monomer feed ratio. In both the A1-1 and CAEM, $v_{\mathrm{C} \equiv \mathrm{N}}$ was at a high wavenumber $\left(2264 \mathrm{~cm}^{-1}\right)$ with lower intensity, which is expected for cyano groups connected to the neutral saturated carbon atom (Figure 3.2).

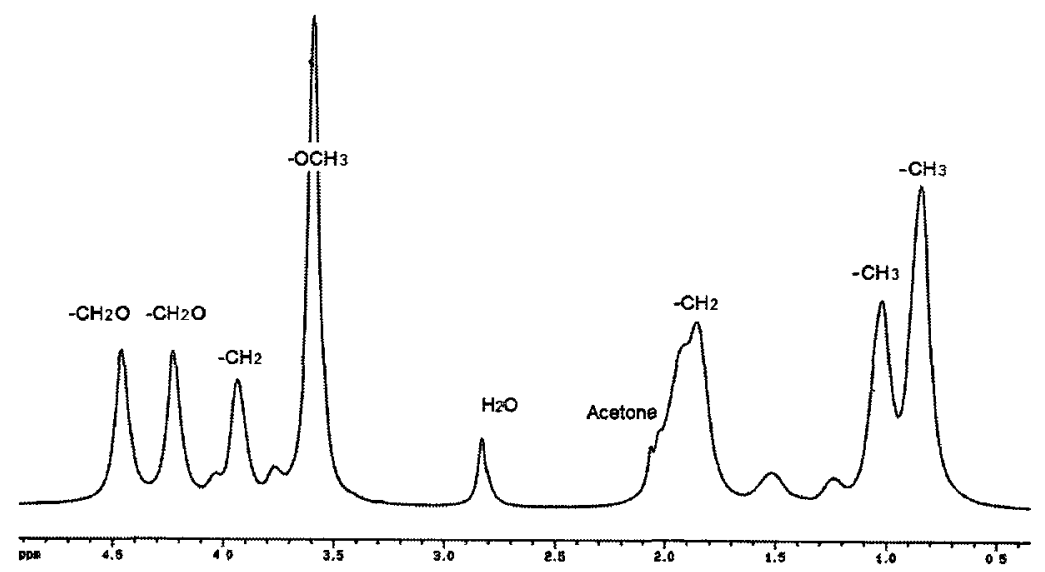

Figure 3.1. ${ }^{1} \mathrm{H}$ NMR spectrum of polymer A1-1 in Acetone-d 6 .

The polymer A1-1 has good solubility in common organic solvents such as acetone, THF, and chloroform. Flexible and transparent films can be readily prepared on indium-tin oxide (ITO) coated glass by solution casting. A1-1 was chosen as a host polymer for FDCN grafting due to its higher CAEM content, which is available for the adjustment of the content of FDCN. Although the $T_{g}$ of A1-1 is lower than the $T_{g}$ of other common used EO polymers, grafting of FDCN chromophores onto the A1-1 backbone was expected to bring the $T_{g}$ up to a temperature suitable for electric poling. 

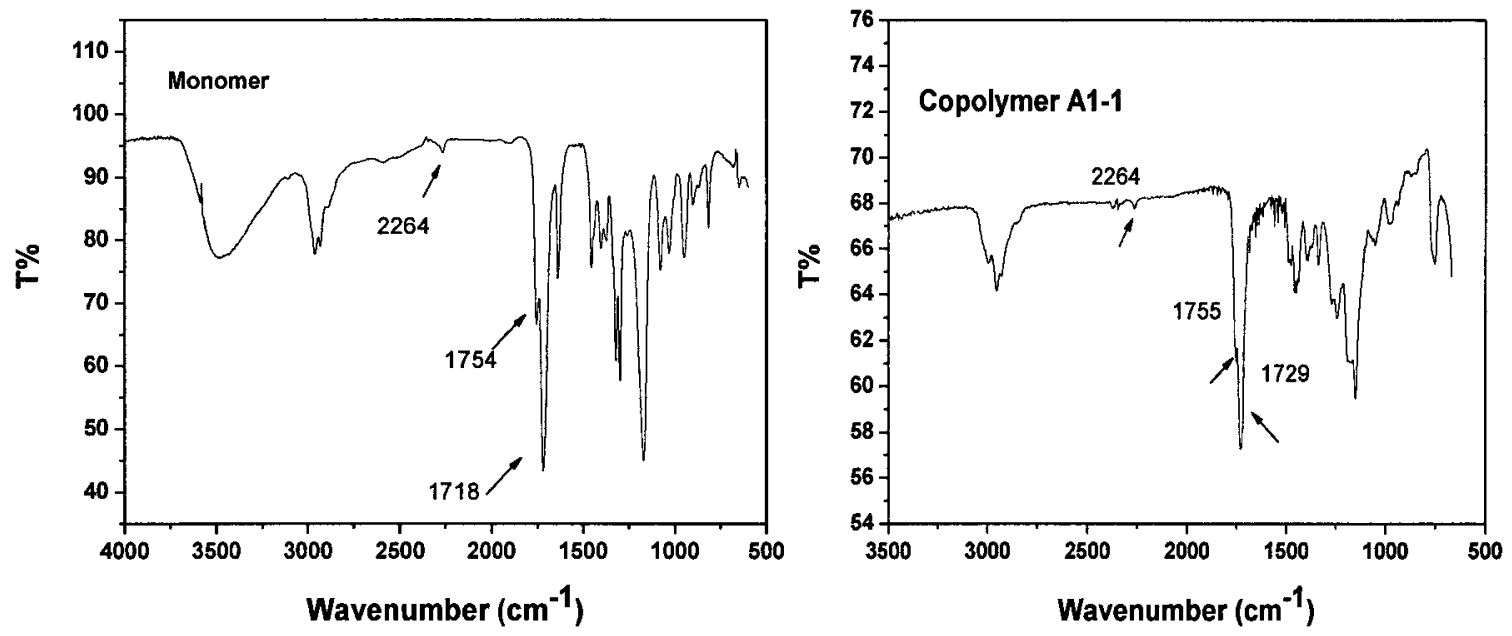

Figure 3.2. IR spectra of CAEM (left) and polymer A1-1(right).

\subsubsection{Synthesis and Characterization of FDCN-A1 NLO Polymers (NA1)}

Scheme 3.2 shows the general synthesis of the FDCN-A1 NLO polymers, NA1-a to NA1-d (Table 3.1) using NaH/THF. Grafting of 2-(4-fluorophenyl)-3-pyridine-4-ylacrylonitrile bromide salt (compound 4, Chapter 2) onto A1-1 polymer was successfully performed at room temperature and confirmed by the IR spectrum of the resulting NLO polymer. The higher reactivity of the cyanoacetate group toward the reaction with compound 4 is expected in view of the electron withdrawing nature of the cyano group, which affects the acidity of the adjacent methylene protons.<smiles>CCCCCC[n+]1ccc(/C=C(\C#N)c2ccc(F)cc2)cc1</smiles>

Scheme 3.2. Synthesis of NLO polymers NA1. 
Table 3.1. Composition and properties of NA1 NLO polymers

\begin{tabular}{cccccc}
\hline Polymer & $\mathrm{X}(\mathrm{mol} \%)^{a}$ & $\mathrm{Y}$ & $\mathrm{Z}$ & $\mathrm{T}_{\mathrm{g}}\left({ }^{\circ} \mathrm{C}\right)^{b}$ & $\mathrm{~T}_{\mathrm{d}}\left({ }^{\circ} \mathrm{C}\right)^{c}$ \\
\hline NA1-a & 0.5 & 31.5 & 68 & 85 & 245 \\
NA1-b & 1.0 & 31.0 & 68 & 86 & 243 \\
NA1-c & 2.0 & 30.0 & 68 & 88 & 240 \\
NA1-d & 5.0 & 27.0 & 68 & 93 & 238 \\
\hline
\end{tabular}

${ }^{a}$ Concentration calculated from a calibration curve established by measuring the absorption of FDCN in THF; ${ }^{b}$ Measured in nitrogen with a heating rate of $10^{\circ} \mathrm{C} \mathrm{min}{ }^{-1}$; ${ }^{c}$ Onset temperature for $5 \%$ weight loss in nitrogen with a heating rate of $10^{\circ} \mathrm{C} \mathrm{min}{ }^{-1}$.

By comparing the IR spectrum of NA1-a (Figure 3.3) with that of A1-1 (Figure 3.2 , right), the position of cyano groups changed noticeably. In $\mathbf{A 1 - 1}, \mathrm{v}_{\mathrm{C} \equiv \mathrm{N}}$ was at a high wavenumber $\left(2264 \mathrm{~cm}^{-1}\right)$ with lower intensity, typical of cyano groups connected to the neutral saturated carbon atom; after the grafting, $v_{\mathrm{C} \equiv \mathrm{N}}$ moved to a lower wavenumber $\left(2171 \mathrm{~cm}^{-1}\right)$ with higher intensity in NA1-a, due to the adjacent negatively charge carbon (characteristic of $\mathrm{C} \equiv \mathrm{N}$ stretch in zwitterionic species).

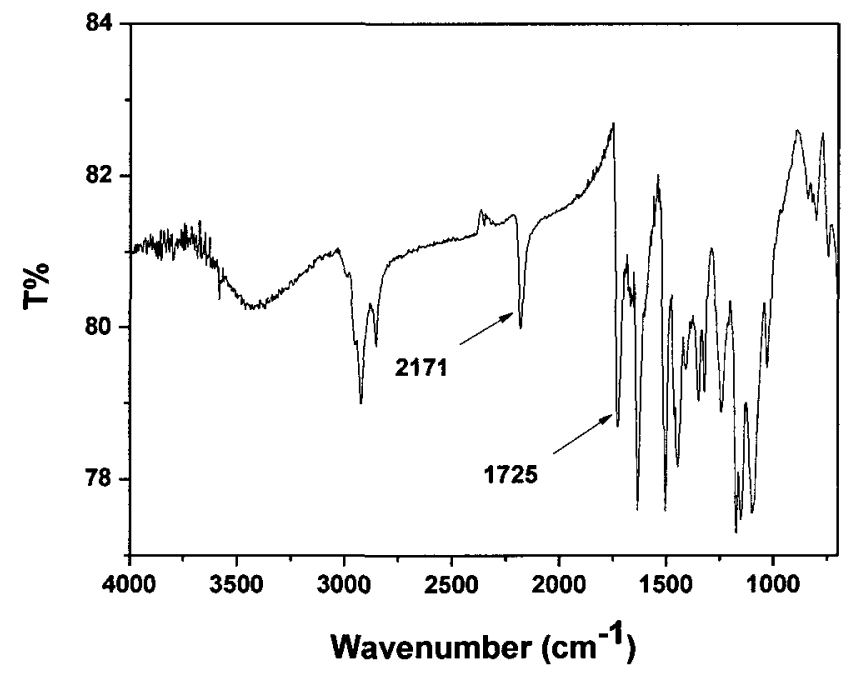

Figure 3.3. IR spectrum of NLO polymer NA1-a.

In comparison with the host copolymer A1, the solubility of NLO polymers NA1 changed dramatically. The NA1 were only soluble in THF, and therefore NMR spectra 
could not be obtained. Transparent thin films were prepared, however, the films became brittle when the FDCN content was increased to above $10 \mathrm{~mol} \%$. EO coefficient was measured under the parallel electrode poling condition, and the $r_{33}$ value is less than 15 $\mathrm{pm} / \mathrm{V}$. The main reason is that the small first hyperpolarizability and low number density of the chromophore in the films lead to the low EO signals.

\subsubsection{Synthesis and Characterization of FDCN-MMA NLO Polymers (NA2)}

To increase the glass transition temperature of the FDCN containing polymer, the flexible cyanoacetated HEMA unit must be eliminated from the polymer backbone. Therefore, FDCN functionalized acrylate was synthesized and copolymerized with MMA monomer. Accordingly, acrylate FDCN monomer (A2) was designed and synthesized by the reaction of FDCNOH, a hydroxyl containing FDCN chromophore with methacryloyl chloride (Scheme 3.3).<smiles></smiles>

Scheme 3.3. Synthesis of acrylate FDCN monomer A2.

The yield of this reaction at room temperature is $70 \%$. In the IR spectrum of $\mathbf{A 2}$, $v_{\mathrm{C} \equiv \mathrm{N}}$ showed at a low wavenumber $\left(2177 \mathrm{~cm}^{-1}\right)$ with a high intensity (Figure 3.4$)$. The maximal absorption of $\mathbf{A 2}$ is at $556 \mathrm{~nm}$ in chloroform, and $496 \mathrm{~nm}$ in DMF (Appendix B, Figure S2.7). This negative solvatochromism indicates that this new FDCN chromophore has the same charge-separated ground state as that in the FDCNOH, which is consistent with the result obtained from IR study. 


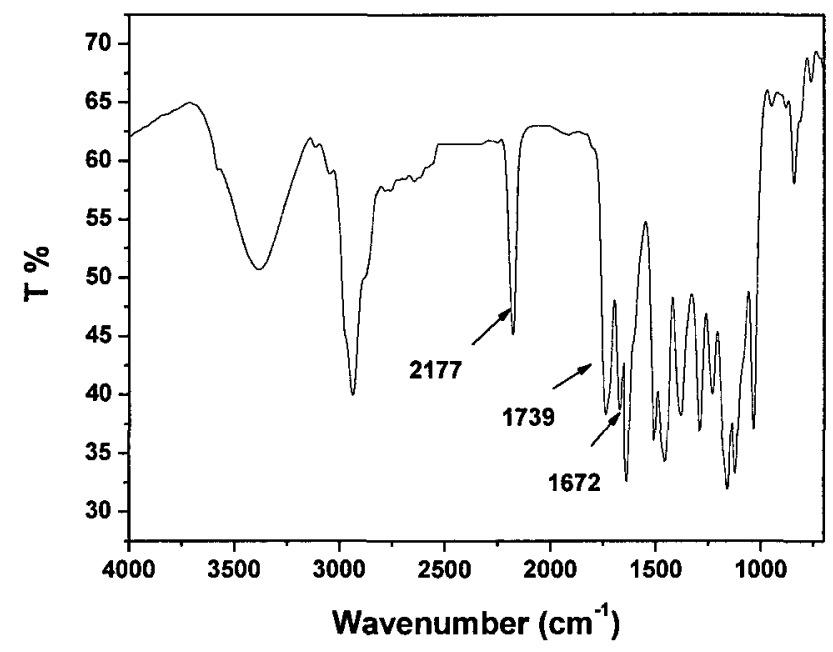

Figure 3.4. IR spectrum of $\mathbf{A} 2$.

The general synthesis of NA2 using the polymerization reaction of $\mathbf{A} 2$ and MMA is shown in Scheme 3.4. The synthesis and characterization of NLO polymers NA2a and NA2b are summarized in Table 3.2. NA2a and NA2b were obtained in 85-90\% yields as light red in powders.

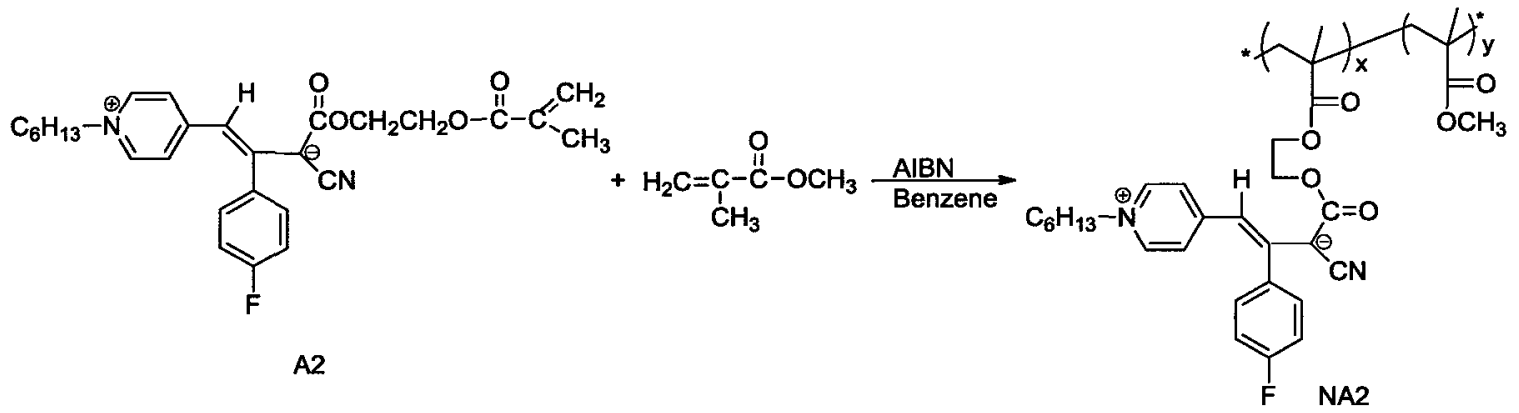

Scheme 3.4. Synthesis of NLO polymers NA2.

Table 3.2. Composition and properties of NA2 polymers

\begin{tabular}{cccccc}
\hline $\begin{array}{c}\text { NLO } \\
\text { Polymer }\end{array}$ & $\begin{array}{c}\mathrm{X}^{a} \\
(\text { wt \%) }\end{array}$ & $\begin{array}{c}\mathrm{Y} \\
(\mathrm{mol} \%)\end{array}$ & $\begin{array}{c}\lambda_{\max } \\
(\mathrm{DMF}, \mathrm{nm})\end{array}$ & $\begin{array}{c}\mathrm{T}_{\mathrm{g}}{ }^{b} \\
\left({ }^{\circ} \mathrm{C}\right)\end{array}$ & $\begin{array}{c}\mathrm{T}_{\mathrm{d}}{ }^{c} \\
\left({ }^{\circ} \mathrm{C}\right)\end{array}$ \\
\hline NA2a & 7 & 95 & 502 & 95 & 264 \\
NA2b & 14 & 90 & 498 & 126 & 232 \\
\hline
\end{tabular}

a Concentration calculated from a calibration curve established by measuring the absorption of FDCN in DMF; ${ }^{b}$ Measured in nitrogen with a heating rate of $10^{\circ} \mathrm{C} \mathrm{min}{ }^{-1}$; ${ }^{c}$ Onset temperature for $5 \%$ weight loss in nitrogen with a heating rate of $10^{\circ} \mathrm{C} \mathrm{min}$. 
The chemical structures of NA2 polymers were consistent with their IR spectra, showing the characteristic peaks of the cyano groups at $2177 \mathrm{~cm}^{-1}$ (Appendix B, Figure S3.3); ${ }^{1} \mathrm{H}$ NMR spectra displaying the expected resonance associated with protons on the structures of both MMA and FDCN (Appendix B, Figure S3.4). The contents of FDCN in the resulting NA2 polymers were calculated from the UV absorption calibration curve and found to be $7 \%$ and $14 \%$. Negative solvatochromic of NA2 was also obtained (e.g., the absorption of NA2a is at $558 \mathrm{~nm}$ in $\mathrm{CHCl}_{3}, 502 \mathrm{~nm}$ in DMF) (Figure 3.5).

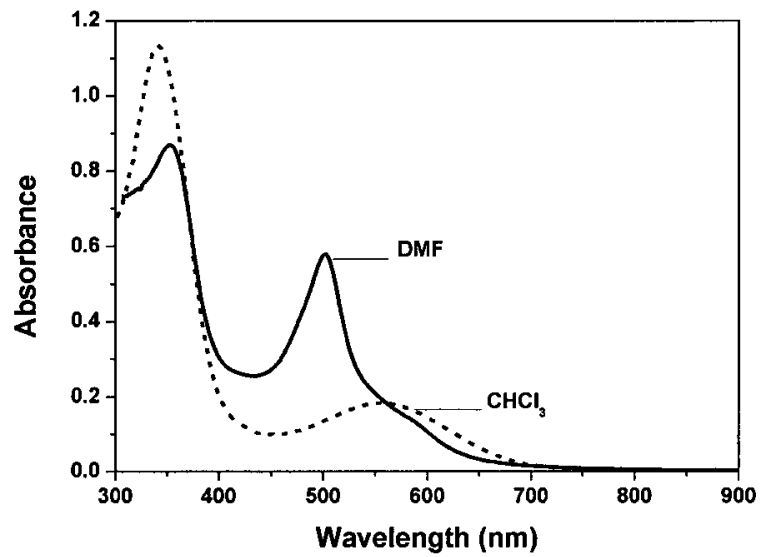

Figure 3.5. UV-Vis spectra of NLO polymers NA2a in DMF and chloroform.

The polymers NA2a and NA2b have good solubility in common organic solvents such as $\mathrm{CHCl}_{3}$, benzene, $\mathrm{ACN}, \mathrm{DMF}$ and have higher $\mathrm{T}_{\mathrm{g}}$ and thermal stability $\left(\mathrm{T}_{\mathrm{d}}>232\right.$ ${ }^{\circ} \mathrm{C}$ ) (Appendix B, Figures S3.5 and S3.6) than the NA1 NLO polymers. Flexible and transparent high quality films were cast on ITO glass from their ACN solutions. The EO properties of these polymers will be discussed in Chapter 4 along with the PpQDM-based crosslinkable NLO polymers. 


\subsection{Synthesis of PpQDM-Based Crosslinkable NLO Polymers}

\subsubsection{Host Polymer Selection for Guest-Host System}

In the search for a suitable host polymer for PpQDM chromophores, high $\mathrm{T}_{\mathrm{g}}$ polymers such as APC, poly(4-hydroxystyrene) (PS-OH), polyvinyl-pyrrolidone (PVP) and PES (Chart 3.1) were screened in a doping test for compatibility with PpQDM. All of the polymers are amorphous and have good film-forming ability.

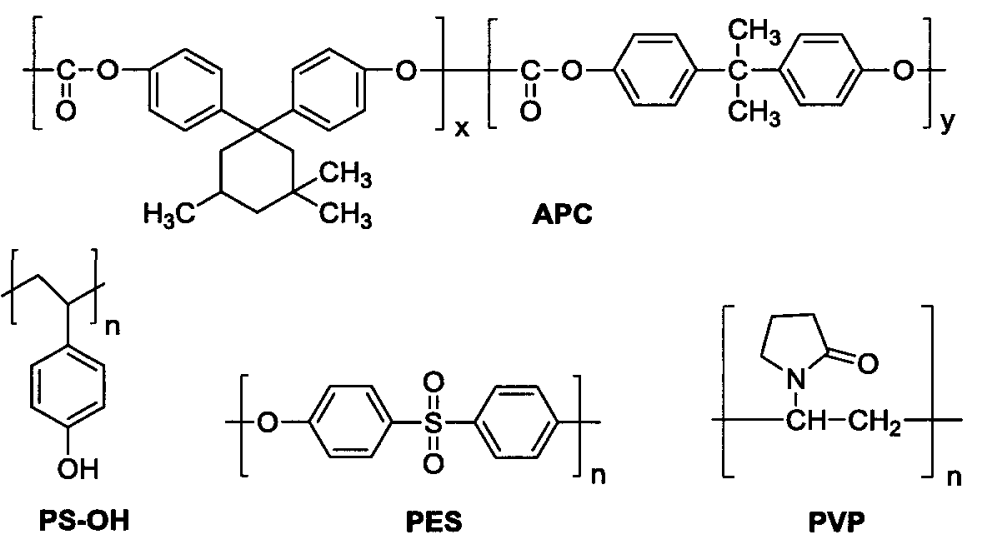

Chart 3.1. Chemical structures of the polymer materials.

PpQDM-Met was doped into these polymers and assessed. Film cracking and serious phase separation were observed in less polar PS-OH and APC (e.g., $\varepsilon$ of APC is 3 ) (Figure 3.6); green color uniform film in PES was obtained ( $\varepsilon=3.5$ ), loading density of PpQDM-Met was lower than that in PVP $(\varepsilon=4.5)$. Among these polymers, the more polar PVP appeared to be the most promising host for high polar PpQDM chromophores. A relatively higher amount of PpQDM-Met ( 3 wt \%) could be blended into PVP (Molecular weight is around $1,300,000$ ) forming a blue color film without showing phase separation. 


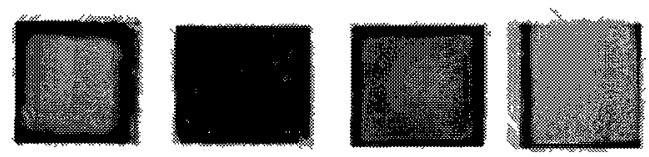

\section{PS-OH 2.APC 3.PVP 4.PES}

Figure 3.6. Films of PpQDM-Met in different polymers. (Color figure can be viewed in the Appendix C, Figure S4.5)

Host polymer selection for PpQDM was also determined by the absorption spectra (Figure 3.7). In comparison with the PpQDM-Met /DMF solution, the charge transfer absorption of PpQDM-Met in APC, PS-OH, PVP doped films underwent negative solvatochromism shifting from $680 \mathrm{~nm}$ to $628 \mathrm{~nm}, 644 \mathrm{~nm}$ and $640 \mathrm{~nm}$, respectively.

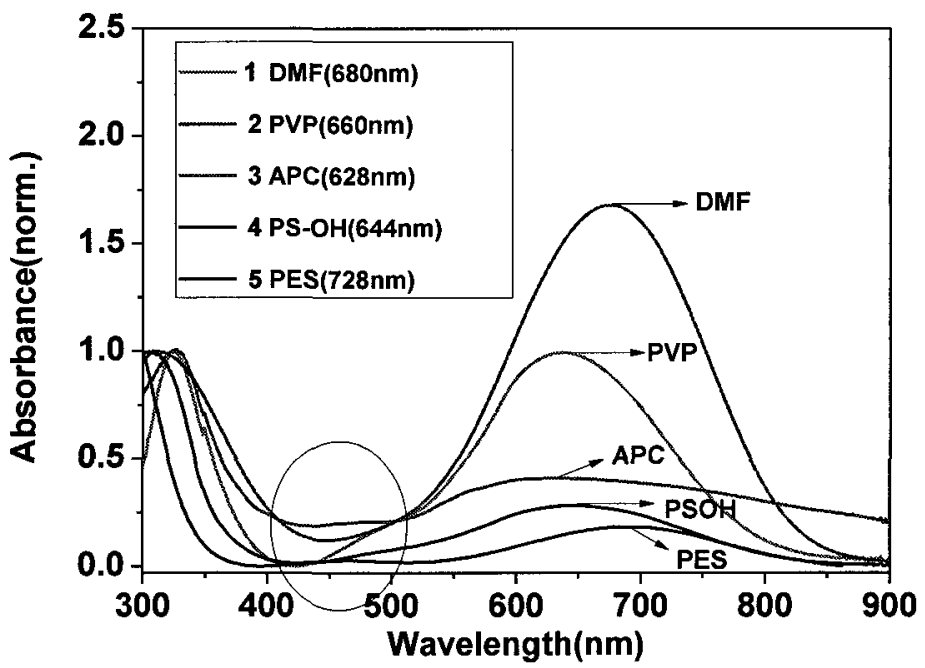

Figure 3.7. Absorption spectra of the films of PpQDM-Met in different polymers.

A red shift of the absorption band was observed for the PES doped film, this implies the existence of $\pi-\pi$ stacking interactions between PpQDM-Met and the host polymer backbone. The broad absorption peaks in APC, PS-OH, PES films and the appearance of a new transition at shorter wavelength $(450 \mathrm{~nm})$ in APC, PS-OH and PES 
confirm that the intermolecular aggregation occurred. ${ }^{11}$ The absorption of PpQDM-Met doped PVP system is close to that in DMF solution, making PVP the best host polymer for PpQDM chromophores. Thus, for further EO measurement, PVP was chosen in our study as a host polymer for PpQDMs guest-host doping system.

\subsubsection{Design and Synthesis of Polyimides for grafting PpQDM Chromophores}

The first challenge for the development of polymers for PpQDM chromophores grafting is the instability of zwitterionic chromophores to some reaction conditions (e.g., acidic, Chapter 2). Therefore, mild reaction conditions should be carefully chosen for grafting reaction. Secondly, PpQDM chromophores have extremely large dipole moments, host polymers with a proper spacer between the functional chromophores must be developed to reduce the electrostatic interactions between chromophores and to allow for good chromophore mobility during the poling process. Thirdly, the host polymers should contain some polar units in the structure and have moderate dielectric constants (e.g., 3.54.5). It is demonstrated that the relatively polar environment leads both to decrease intermolecular electrostatic interactions and to enhance the poling efficiency. Additionally, since PpQDM is ready functionalized with a hydroxyl group (e.g., PpQDM-C3OH), host polymers contains carboxylic acid groups is expected to allow for an efficient coupling reaction under mild reaction conditions.

A wide range of linear polyimides containing carboxylic acid with different glass transition temperatures and chain mobilities were designed and developed in our group. Among these polymers, poly(ether imide)s were promising host candidates for the highly polar PpQDM chromophores to obtain NLO polymers with large EO activity and good auxiliary properties. It was found that PI-1 possessed good chain mobility, suitable $\mathrm{T}_{\mathrm{g}}$ 
$\left(208-227^{\circ} \mathrm{C}\right)$ and molecular weights $\left([\eta]_{\mathrm{nnh}}=0.22-0.37 \mathrm{dL} / \mathrm{g}\right)$ with good solubility, thermal stability, and film-forming ability. Most importantly, the dielectric constants of PI-1s were in the range of $3.5-4,{ }^{12}$ which meet the requirement of the polarity of the polymer matrix for PpQDM chromophores.

The reaction conditions were optimized by a former graduate (Dr. Naiheng Song) through investigating carefully the effect of backbone composition, end-capping groups and different feed ratios. ${ }^{12}$ Using 1-(3-(dimethylamino)propyl)-3-ethylcarbodiimide (EDC) as a coupling agent, a series of NLO polyimides can be prepared.

The general synthesis of PI-1 is shown in Scheme 3.5. The results of synthesis and characterization of PI-1a - PI-1d are summerized in Table 3.3. A relatively flexible diamine, 3, 5-bis (4-aminophenoxy) benzoic acid (DAPBA) was synthesized and polymerized with $4,4^{\prime}$ '-(4, 4'-isopropylidenediphenoxy)bis(phthalic anhydride) (BPADPA) in the presence of phthalic anhydride (PA) (2-20 mol\% relative to DAPBA) as an end-capping agent to afford the host PI-1.

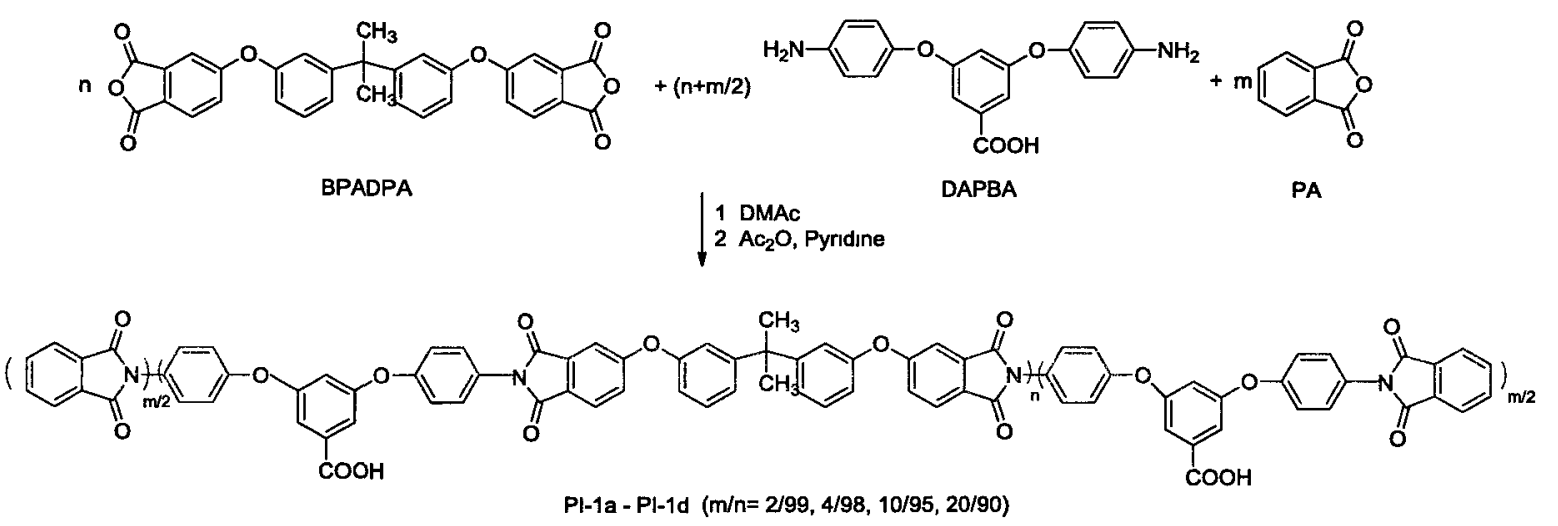

Scheme 3.5. Poly(ether imide) PI-1 derived from DAPBA. 
Table 3.3. Synthesis and characterization of poly (ether imide)s PI-1

\begin{tabular}{|c|c|c|c|c|c|}
\hline \multirow[b]{2}{*}{ Polymer } & \multicolumn{2}{|c|}{ Feed Ratio (mol \%) } & \multirow{2}{*}{$\begin{array}{c}\mathrm{T}_{\mathrm{g}} \\
\left({ }^{\circ} \mathrm{C}\right)\end{array}$} & \multirow{2}{*}{$\begin{array}{l}{[\eta]_{\text {inh }}} \\
(\mathrm{dL} / \mathrm{g})\end{array}$} & \multirow{2}{*}{$\begin{array}{c}\mathrm{T}_{\mathrm{d}} \\
\left({ }^{\circ} \mathrm{C}\right)\end{array}$} \\
\hline & BPADPA & $\mathrm{PA}$ & & & \\
\hline PI-1a & 0.99 & 0.02 & 227 & 0.37 & 450 \\
\hline PI-1b & 0.98 & 0.04 & 211 & 0.33 & 450 \\
\hline PI-1c & 0.95 & 0.10 & 210 & 0.30 & 453 \\
\hline PI-1d & 0.90 & 0.20 & 208 & 0.22 & 451 \\
\hline
\end{tabular}

The polymerization was completed by a conventional two-step method, i.e., the formation of poly (amic acid)s and subsequent chemical imidization in the presence of acetic anhydride and pyridine. The ${ }^{1} \mathrm{H}$ NMR and IR spectra of PI-1 were consistent with the polymer structure (Appendix B, Figures S3.7 and S3.8).
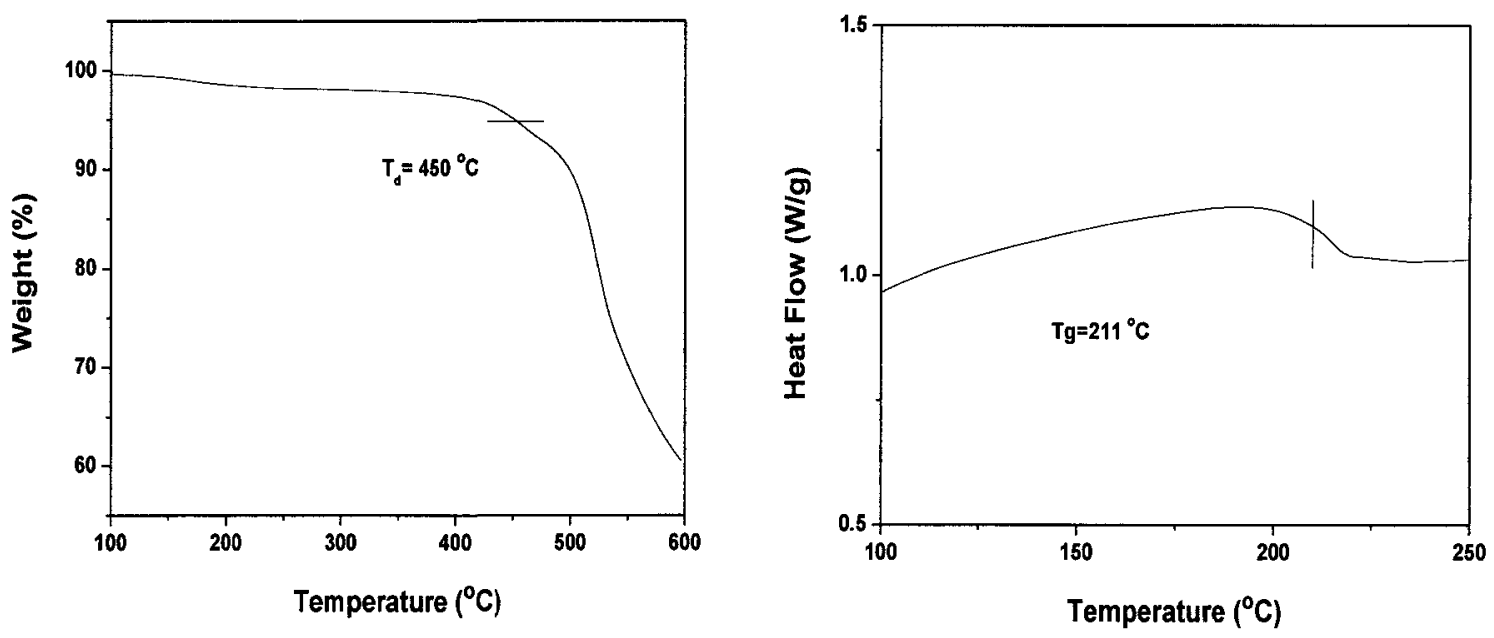

Figure 3.8. TGA (left) and DSC (right) traces of polyimide PI-1b.

PI-1b was chosen as the host polymer for chromophore grafting to prepare the NLO polymer due to the moderate $\mathrm{T}_{\mathrm{g}}$ and good solubility. The molecular weight of PI-1b was not directly measured; it was estimated to be high enough with a high viscosity $(0.33$ 
$\mathrm{dL} / \mathrm{g})$. PI-1b can be precipitated from methanol as fibers and was readily soluble in THF, $\mathrm{N}, \mathrm{N}$-dimethylacetamide (DMAc), and DMF, and could be cast into flexible tough films on glass. TGA analysis indicated that PI-1b has a good thermostability with $T_{d}$ of $450{ }^{\circ} \mathrm{C}$ for $5 \%$ weight loss in nitrogen (Figure 3.8 left) and a moderate $\mathrm{T}_{\mathrm{g}}$ of $211{ }^{\circ} \mathrm{C}$ (Figure 3.8 right).

\subsubsection{Design and Synthesis of PpQDM-Based Crosslinkable NLO Polymers}

In general, noncentrosymmetric order in poled polymers tends to decay very quickly in low- $\mathrm{T}_{\mathrm{g}}$ polymers at ambient to elevated temperatures. Accordingly, chromophores are usually incorporated into high- $T_{\mathrm{g}}$ polymers to improve temporal stability of EO activity. However, if the $\mathrm{T}_{\mathrm{g}}$ of a polymer is too high, a higher poling temperature will be required which may cause chromophore decomposition. One of the most effective approaches to improve thermal stability of EO materials is to introduce covalent cross-linking system. Crosslinkable NLO polymers can be poled and crosslinked consecutively to lock-in the poling-induced nonlinearity.

In early studies of organic EO materials, such crosslinking was normally accomplished by utilizing condensation reactions to form polyurethane between the $\mathrm{OH}$ and NCO groups. ${ }^{13}$ More recently, cycloaddition reactions have largely replaced these earlier methods. The two commonly utilized cycloaddition reactions include 1) reaction of fluorovinyl ether groups to form cyclobutyl crosslinks ${ }^{14}$ and 2) Diels-Alder/retroDiels-Alder reaction of dienes and dienophiles. ${ }^{15}$ The latter has become very popular because of the wide range of diene and dienophiles available, which can be used to widely tune the glass transition temperatures of materials. 
A new thermally reactive compound, 5-aminobenzocyclobutenone (BCBO), was developed by our group ${ }^{16}$ and it has been demonstrated that the BCBO group in a variety of polymers or by itself can undergo a ring-opening reaction at temperatures above $200{ }^{\circ} \mathrm{C}$ to form a reactive vinylketene intermediate (Scheme 3.6). The subsequent dimerization of the vinylketene as side groups on polymer chain by self [4+2] cycloaddition and a 1,5-hydrogen shift lead to the polymer cross-linking or curing. ${ }^{6 b, 7 \mathrm{c}}$ Therefore crosslinkable NLO polyimides can be obtained through grafting the hydroxylcontaining PpQDM and BCBO crosslinker onto the PI-1b backbones.

2<smiles>C=c1ccccc1=C=O</smiles>

Scheme 3.6. Thermolysis and dimerization of BCBO.

\subsubsection{Synthesis of PpQDM-Based Crosslinkable NLO Polymers}

Grafting of PpQDM-C3OH onto PI-1b was performed by using EDC and 4dimethylaminopyridine (DMAP). ${ }^{17}$ Although PpQDMs are labile to acetic acid, they appeared to be stable to acid-containing polyimides, particularly in the presence of organic base such as pyridine or DMAP. Since covalently linking PpQDM-C3OH as a pendent group will largely increase the polarity of the polymer, ${ }^{18}$ in addition with the strong interaction between the PpQDM chromophores, blocking groups with less polarity than PpQDM-C3OH should be introduced into the polymer to block the chromophore interaction to allow the NLO polymer to have a suitable $\mathrm{T}_{\mathrm{g}}$ for poling without causing any decomposition. A preliminary study on the $\mathrm{T}_{\mathrm{g}}$ of two NLO polymers with different ratio of $\mathrm{PpQDM}-\mathrm{C} 3 \mathrm{OH}$, crosslinkable $\mathrm{BCBO}$, and blocking pendent groups (methanol or 
1-hexanol) was carried out in order to identify an appropriate composition for NLO polymers. The difference of the short and long aliphatic blocking groups is expected to affect the $T_{g}$ of NLO polyimides.

Scheme 3.7 shows the general synthesis of NLO polyimides (NPI-a-NPI-e). The reactions were carried out at room temperature in DMAc by adding EDC to a mixture of PI-1b, PpQDM, BCBO, methanol or 1-hexanol. The structures of the synthesized PpQDM-grafted NLO polyimides, NPI-a to NPI-d are shown in Chart 3.2.

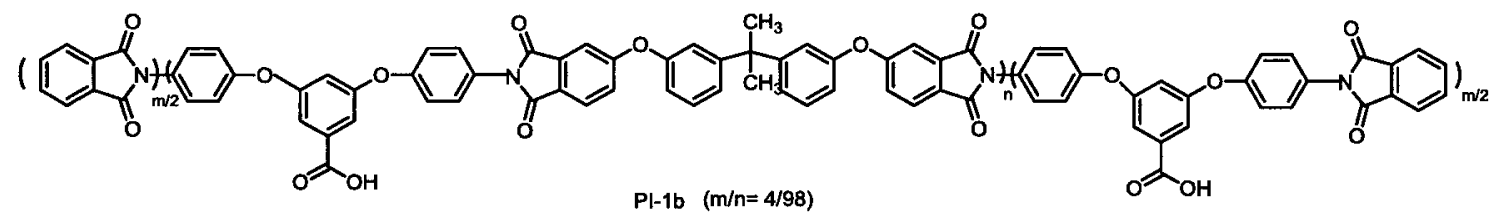

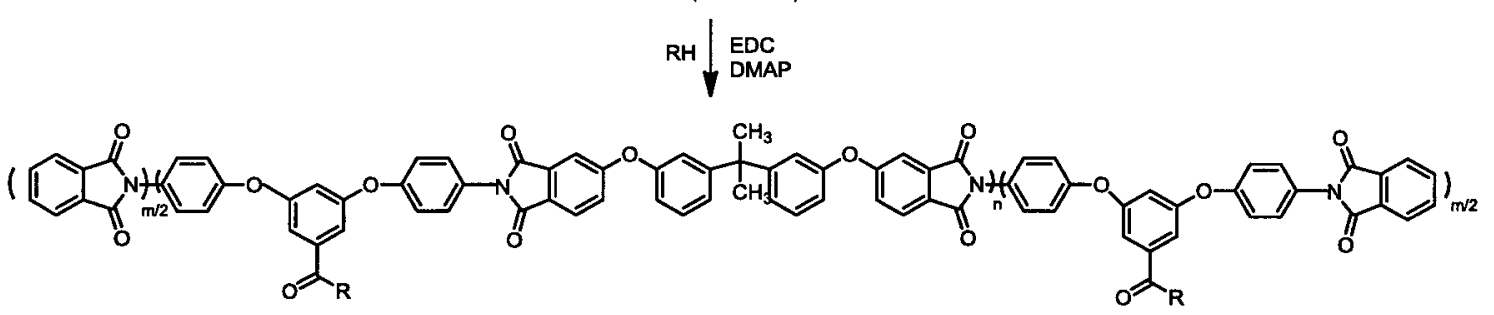

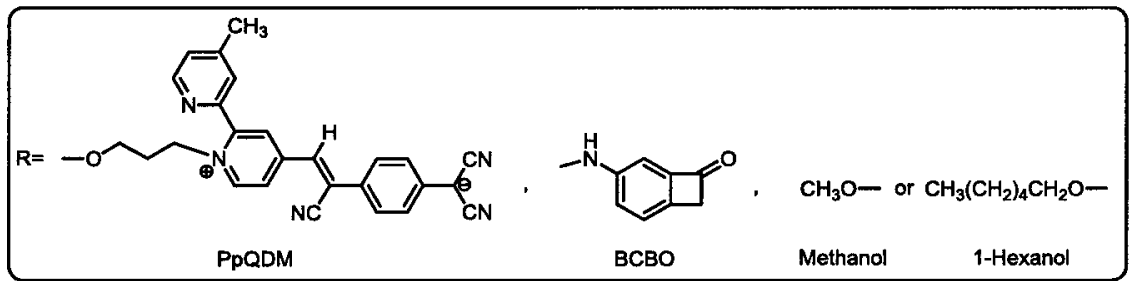

Scheme 3.7. Synthesis of NLO polyimides.

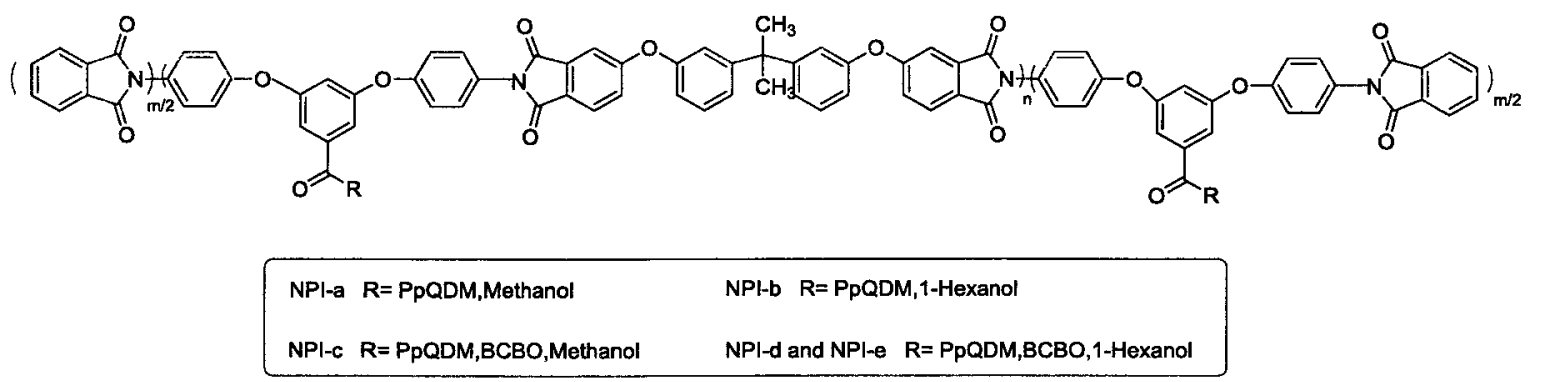

Chart 3.2. Structures of NLO polyimides NPI-a to NPI-d. 
Table 3.4 summarizes the synthesis and characterization of NLO polyimides NPIa - NPI-e. All NLO polymers were prepared from the same batch of host polyimide PI1b. These NLO polymers differ in the PpQDM content, the thermally reactive BCBO unit content and the end-capping groups.

Table 3.4. Synthesis and characterization of crosslinkable NPI polymers

\begin{tabular}{llllll}
\hline $\begin{array}{l}\text { NLO } \\
\text { Polyimide }\end{array}$ & $\begin{array}{l}\mathrm{PpQDM}^{c} \\
(\mathrm{wt} \%)\end{array}$ & $\begin{array}{l}\mathrm{BCBO}^{d} \\
(\mathrm{~mol} \%)\end{array}$ & $\begin{array}{l}\lambda_{\max } \\
(\mathrm{DMF}, \mathrm{nm})\end{array}$ & $\begin{array}{l}\mathrm{T}_{\mathrm{g}}{ }^{e} \\
\left({ }^{\circ} \mathrm{C}\right)\end{array}$ & $\begin{array}{l}\mathrm{T}_{\mathrm{d}}{ }^{f} \\
\left({ }^{\circ} \mathrm{C}\right)\end{array}$ \\
\hline NPI-a $^{a}$ & 2.62 & 0 & 702 & 223 & 265 \\
NPI-b $^{b}$ & 4.71 & 0 & 695 & 185 & 264 \\
NPI-c $^{a}$ & 7.12 & 30 & 695 & 200 & 260 \\
NPI-d $^{b}$ & 7.59 & 5 & 694 & 163 & 255 \\
NPI-e $^{b}$ & 13.75 & 5 & 695 & 180 & 253
\end{tabular}

${ }^{a}$ The residual acid residues were blocked with methanol. ${ }^{b}$ The residual acid residues were blocked with 1 -Hexanol. ${ }^{c}$ PpQDM content in weight percentage calculated from a calibration curve established by measuring the absorption of PpQDM-C3OH in DMF ${ }^{d}$ Molar ratio relative to the acid groups in the polymers. ${ }^{e}$ Measured by DSC in nitrogen $\left(10{ }^{\circ} \mathrm{C} \mathrm{min}{ }^{-1}\right) .{ }^{f}$ Onset temperature for $5 \%$ weight loss measured by TGA in nitrogen.

Both NPI-a and NPI-b have high thermo stability with a decomposition temperature close to $265^{\circ} \mathrm{C}$, indicating both the methyl ester and hexyl ester bond are thermally stable. As expected, the $\mathrm{T}_{\mathrm{g}}$ of NPI-b is much lower than that of NPI-a, by changing the length of the side chain, going from one carbon to six carbons, the $T_{g}$ of the polymer decreases from $223{ }^{\circ} \mathrm{C}$ to $185^{\circ} \mathrm{C}$, despite the fact that NPI-b has higher rigid chromophore content than that of NPI-a. One can infer from this that introducing the softer long alkyl side chain can effectively lower the $T_{g}$ of the NLO polymer. Therefore, 
1-hexanol was chosen as the end-capping group for the synthesis of PpQDM-Based crosslinkable NLO polymers.

Previous studies indicated that a small amount of $\mathrm{BCBO}$, such as $5 \mathrm{~mol} \%$ introduced onto host polymers as a crosslinker can effectively form crosslinkable NLO polymers. ${ }^{12,18}$ Therefore, the BCBO content was lowered from $30 \mathrm{~mol} \%$ in NPI-c to 5 mol\% in NPI-d and NPI-e. A suitable NLO polymer, NPI-e, was obtained with the highest chromophore content $(13.8 \mathrm{wt} \%)$ and a high $\mathrm{T}_{\mathrm{g}}\left(180^{\circ} \mathrm{C}\right)$.

The NLO polyimides were obtained in $80-92 \%$ yields, NPI-a to NPI-c were light blue-green and NPI-d to NPI-e were deep blue. These crosslinkable polymers were characterized by IR, ${ }^{1} \mathrm{H}$ NMR and UV-Vis methods.

The change in chemical structure of NPI-a to NPI-e was consistent with their IR spectra. For NPI-e, Figure 3.9 showing the characteristic peaks of the cyano groups at 2178 and $2138 \mathrm{~cm}^{-1}$ and of the imides at 1777 and $1720 \mathrm{~cm}^{-1}$.

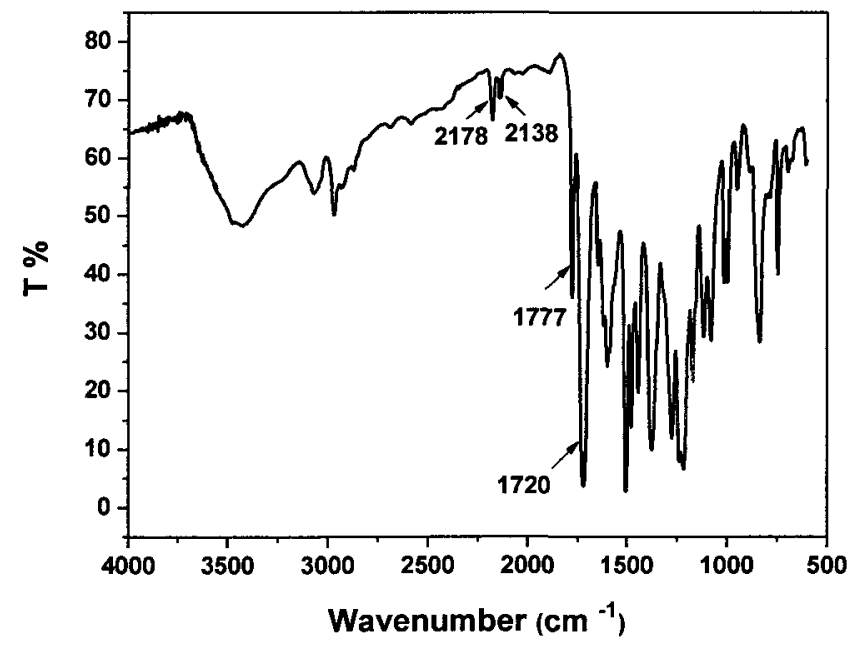

Figure 3.9. IR spectrum of NPI-e (KBr pellet). 
${ }^{1} \mathrm{H}$ NMR spectra of NLO polyimides, displayed the expected resonance associated with protons on the structures of host polyimides, $\mathrm{PpQDM}, \mathrm{BCBO}$ and $\mathrm{ROH}$. In Figure 3.10, the unlabeled peaks represent the polymer main chain and the hexyl group in NPI-e. The total content of PpQDM in NLO polymers was estimated from the ${ }^{1} \mathrm{H}$ NMR spectra to be in the range of 5-26.25 mol \% relative to the acid groups in the host polymers. The actual content was determined by UV-Vis analysis to be in the range of $2.6-13.8 \mathrm{wt} \%$. The chromophore loading in NPI-e is higher than the guest-host system, in which the highest doping level of PpQDM chromophores in PVP is 5 wt \%.

All the NLO polyimides were soluble in polar aprotic solvents such as DMF, DMAc, DMSO and NMP. Films of NPI-a - NPI-e in thickness of 1-3 $\mu \mathrm{m}$ can be readily prepared by casting of their solutions in DMF. Transparent films can be made on ITO glass. Good stability or dispersion of PpQDM in NLO polyimide films indicates a successful design of the host polymer in terms of achieving a high guest number density.

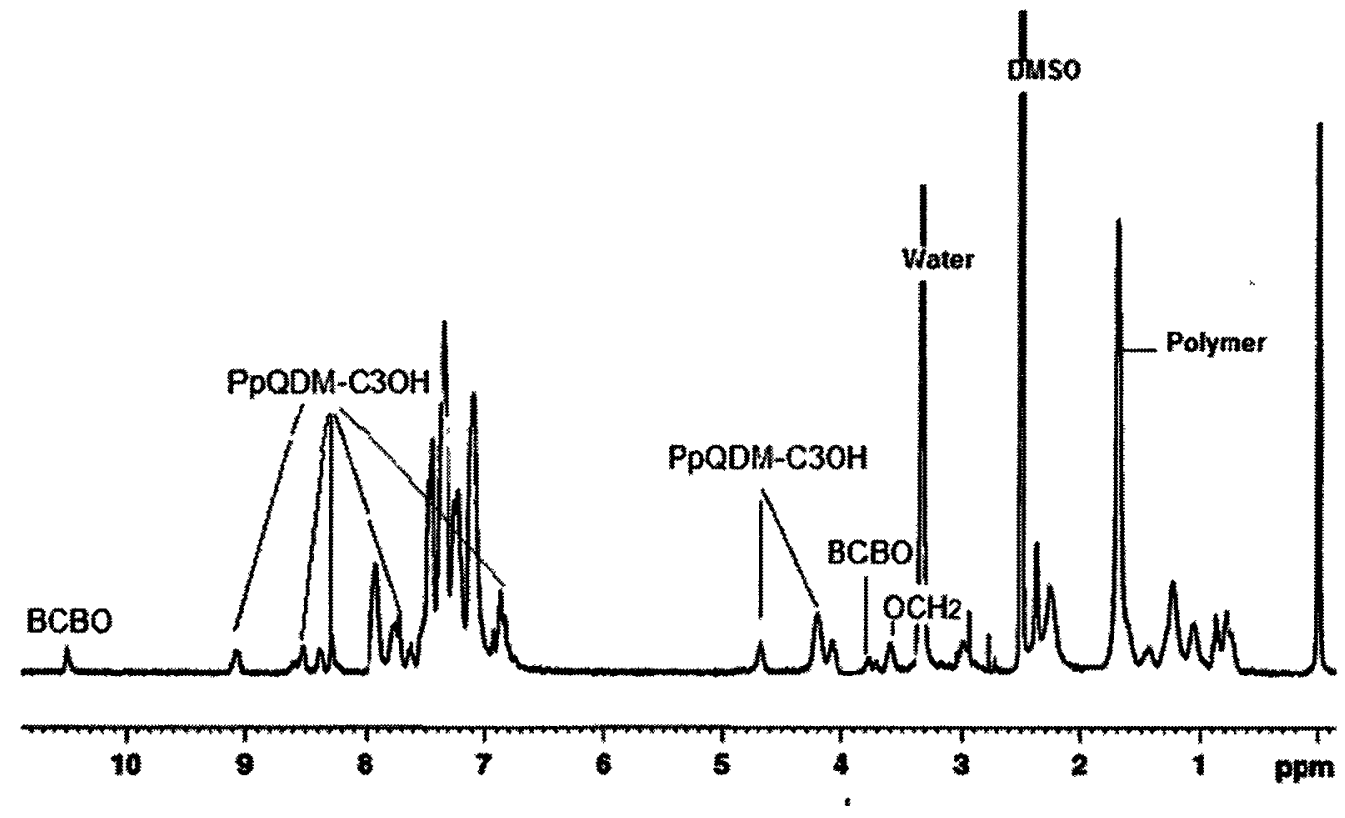

Figure 3.10. NMR spectrum of NPI-e in DMSO- $d_{6}$ 


\subsubsection{Optical Absorption}

A slight shift (10 -17 $\mathrm{nm}$ ) of the charge transfer band to longer wavelengths was observed for the NLO polyimides in DMF solution when compared with that of PpQDM$\mathrm{C} 3 \mathrm{OH}(685 \mathrm{~nm})$. For example, NPI-b in DMF solutions showed a bathochromic shift of about $10 \mathrm{~nm}$ in the CT absorption and NPI-a showed a larger shift of $17 \mathrm{~nm}$ (Figure 3.11 , Table 3.4). Since the intramolecular CT absorption of organic molecules is related to the electronic environment, this red-shift in absorption may be due to less polar environment imparted by the polyimide backbones.

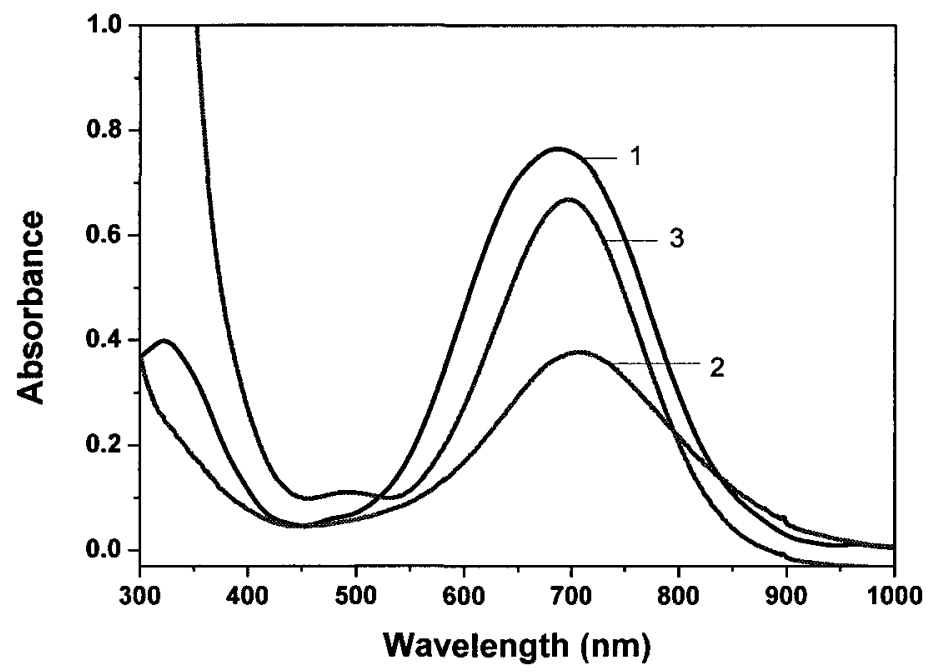

Figure 3.11. UV-Vis absorption spectra in DMF: 1) PpQDM-C3OH 2) NPI-a 3) NPI-b.

Although NPI-a to NPI-e show similar UV-Vis absorption spectra in DMF solution, the spectra of their corresponding films are apparently different. In comparison with the absorption of NLO polyimides in DMF solution, the CT absorption of NLO polyimide films showed a distinct peak broadening $(\sim 200 \mathrm{~nm})$ and a bathochromic shift (Figure 3.12). The absorption peak of the NLO polymers is shifted to longer wavelengths as compared with the chromophore in DMF solution due to the lower polarity of the chromophore environment. The CT bond of NPI-e is much broader than that of NPI-b. 
The broad peak is due to some J-aggregation of high loading of chromophores in less polar polymer environment. ${ }^{18}$

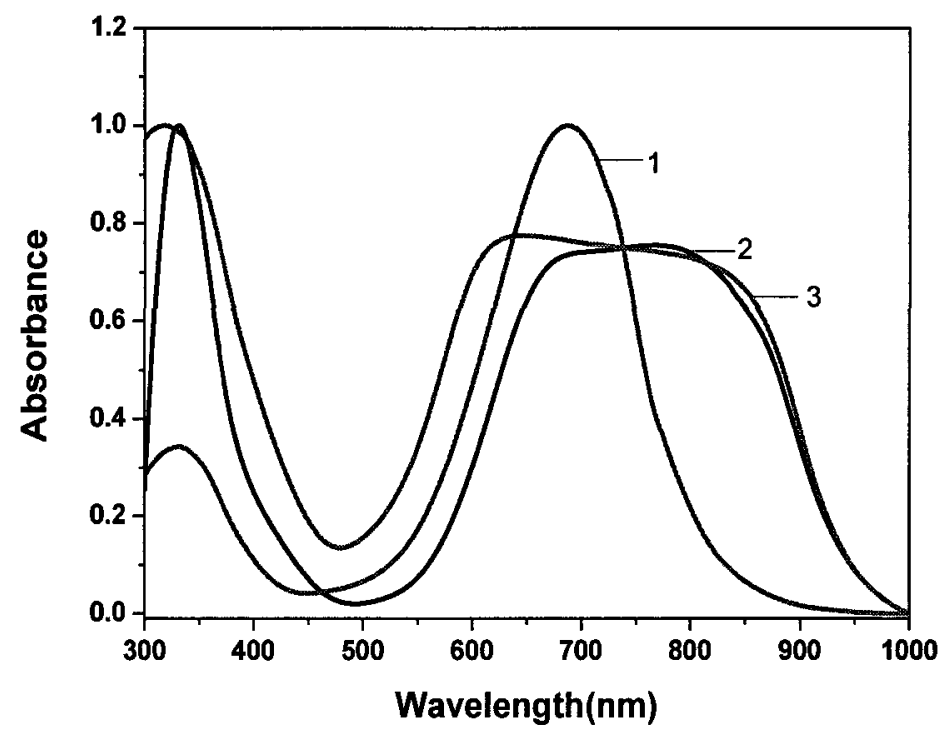

Figure 3.12. UV-Vis absorption spectra of a solution of PpQDM-C3OH in DMF (1), films of NPI-b (2) and NPI-e (3).

\subsubsection{Thermal Analysis}

In order to find applications in EO devices, NLO polymers must be thermally stable to the material processing temperature, poling temperature, and device processing and packaging temperatures. Thermal properties of NPI-a to NPI-e were measured by DSC. NPI-c containing higher BCBO content shows higher $\mathrm{T}_{\mathrm{g}}\left(20{ }^{\circ} \mathrm{C}\right)$ compared to NPI-d $\left(163{ }^{\circ} \mathrm{C}\right)$. The higher $\mathrm{T}_{\mathrm{g}}$ of NPI-e compared to NPI-d is due to higher chromophore content. Figure 3.13 shows the DSC trace of NPI-e, after the glass transition, an endothermic process was found around $187^{\circ} \mathrm{C}$, followed by an exothermic process starting at $198{ }^{\circ} \mathrm{C}$. The exothermic process is due to the ring-opening of the BCBO group. 


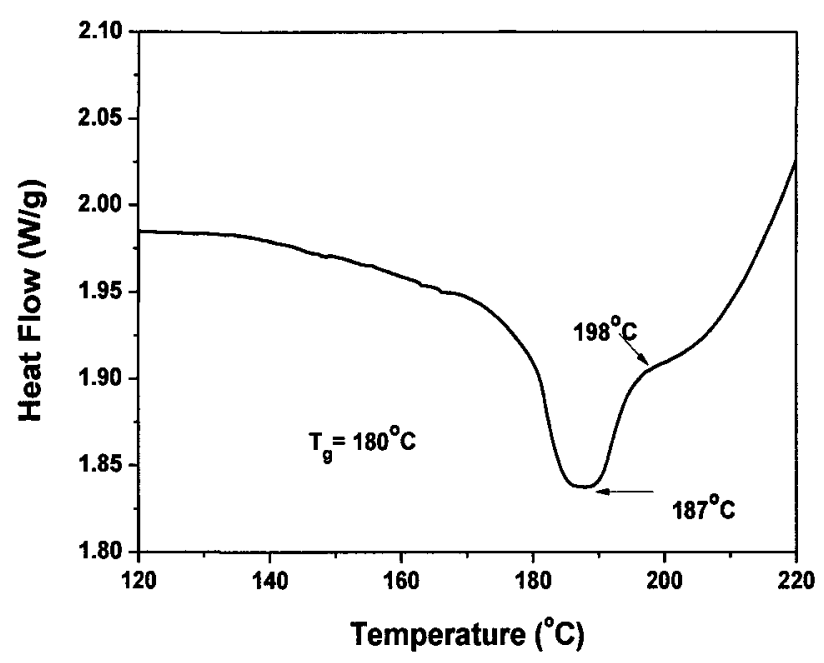

Figure 3.13. Representative DSC trace of NPI-e with a heating rate of $10^{\circ} \mathrm{C} \mathrm{min}-1$.

The thermal stability of NPI-a to NPI-e was determined by TGA in nitrogen. Figure 3.14 compares the TGA traces between PpQDM-C3OH and NLO polymer NPI-a to NPI-c and NPI-e. All the polymers showed good thermal stability with the $T_{d}$ above $250{ }^{\circ} \mathrm{C}$, which are comparable to that of PpQDM-C3OH $\left(283^{\circ} \mathrm{C}\right)$. These $\mathrm{T}_{\mathrm{g}}$ and $\mathrm{T}_{\mathrm{d}}$ data were used to determine the suitable poling temperature and decomposition point for the process of EO measurements.

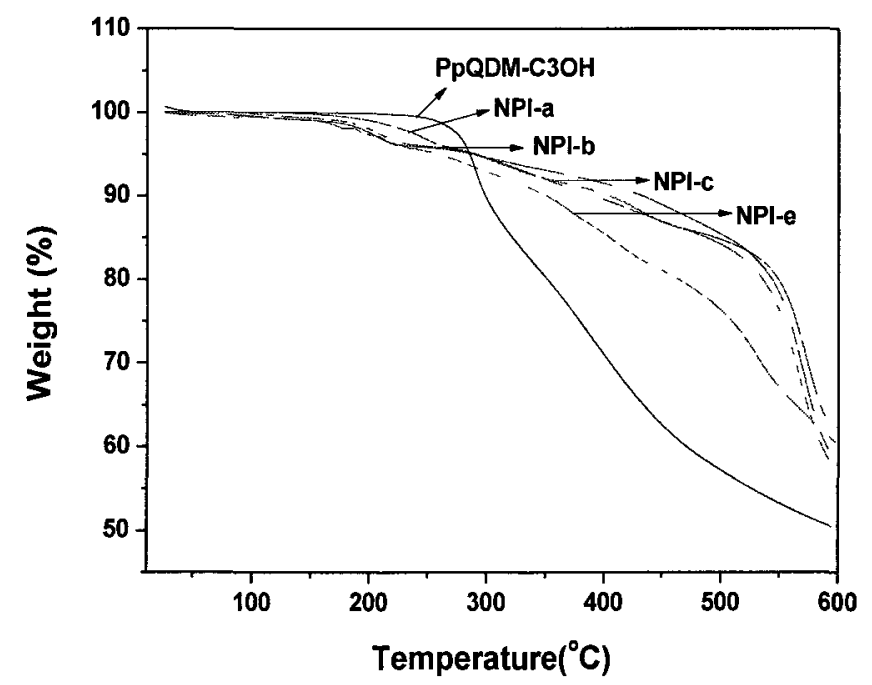

Figure 3.14. TGA traces of PQDM-C3OH, NPI-a - NPI-c and NPI-e. 


\subsection{Conclusions}

Based on the results of preliminary compatibility test, PMMA and PES were selected to be the host polymers for the doping of FDCN. Two series of FDCN-based NLO polymers, NA1 and NA2 were prepared by either connecting the FDCN chromophores by a post-polymerization reaction or copolymerizing a FDCN containing monomer with MMA, respectively. The solubility of NA1 is limited to THF only and the films became brittle when increasing the content of FDCN chromophore to above $10 \%$. EO coefficient was less than $15 \mathrm{pm} / \mathrm{V}$ due to the low number density of the chromophore in the films and low $T_{g}$ of the NLO polymers. In comparison with NA1, NA2 has higher FDCN content (up to $14 \mathrm{wt} \%$ ) and higher $\mathrm{T}_{\mathrm{g}}$. It has good solubility in common organic solvents such as $\mathrm{CHCl}_{3}$, benzene, $\mathrm{ACN}$ and DMF. NA2 can form high quality films for EO measurement and have high thermal stability $\left(\mathrm{T}_{\mathrm{d}}>232{ }^{\circ} \mathrm{C}\right)$.

Based on the comparative studies of chromophore doping in various polymers, PVP was selected to be the host polymer for doping the highly polar PpQDM chromophore. Poly(ether imide), PI-1 was chosen for grafting with PpQDM chromophore. A series of acid-containing linear polyimides (PI-1a - PI-1d) having different $\mathrm{T}_{\mathrm{g}}\left(208-227^{\circ} \mathrm{C}\right)$ and molecular weights $\left([\eta]_{\mathrm{inh}}=0.22-0.37 \mathrm{dL} / \mathrm{g}\right)$ were prepared. The acid groups in host polyimides allow for chemical grafting of the hydroxylcontaining PpQDM.

A series of PpQDM-based crosslinkable NLO polyimides (NPI-a - NPI-e) were synthesized in 80-92\% yields through grafting PpQDM-C3OH onto PI-1b. The effect side chains on the $T_{g}$ of the polymer and preliminary investigation into the chromophore loading are discussed. The reactive $\mathrm{BCBO}$ group was introduced to the side chains to 
enable polymer crosslinking. Covalently grafting $\mathrm{PpQDM}-\mathrm{C} 3 \mathrm{OH}$ onto a polyimide can increase the chromophore loading level to a certain up to $13.8 \mathrm{wt} \%$ overall. Thermal crosslinking was performed at temperatures around $198{ }^{\circ} \mathrm{C}$, which is higher or comparable to the $\mathrm{T}_{g}$ of most NLO polyimides. The $\mathrm{T}_{g}$ of NPI-a to NPI-e was observed to vary due to different pendant groups (methanol or 1-hexanol) and different PpQDM loading level. PpQDM-based crosslinkable NLO polymers were found to have good filmforming ability and high thermal stability $\left(\mathrm{T}_{\mathrm{d}}>250^{\circ} \mathrm{C}\right)$.

\subsection{Experimental Section}

Materials. HEMA, DCC, cyanoacetic acid, CAEM, MMA, cyanoacetic acid, 2hydroxyethyl alcohol, EDC and DMAP were purchased from Aldrich and used as receieved. AIBN was purchased from Aldrich and recrystallized from methanol. DMAc was dried over calcium hydride, vacuum distilled, and stored over $4 \AA$ molecular sieves. Other chemical reagants were purchased from Aldrich and used as received.

Methods. Solubility tests were carried out by dissolving about $10 \mathrm{mg}$ of polymer in $1 \mathrm{~mL}$ of studied solvent. For general characterization methods, such as ${ }^{1} \mathrm{H}$ and ${ }^{13} \mathrm{C} \mathrm{NMR}, \mathrm{MS}$, IR, UV-Vis, DSC and TGA, refer to Chapter 2.

\section{Synthesis of CAEM}

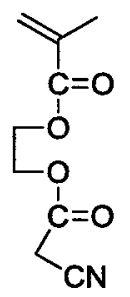

To a stirred solution of 2-hydroxyethyl methacrylate $(6.5 \mathrm{~g}, 50 \mathrm{mmol})$ and cyanoacetic acid $(4.45 \mathrm{~g}, 52.5 \mathrm{mmol})$ dissolved in dry $\mathrm{CH}_{2} \mathrm{Cl}_{2}(30 \mathrm{~mL})$ under $\mathrm{N}_{2}$ and 
cooled with an ice bath was added DCC $(10.8 \mathrm{~g}, 52.5 \mathrm{mmol})$ dissolved in $\mathrm{CH}_{2} \mathrm{Cl}_{2}(30$ $\mathrm{mL}$ ). A white precipitate formed, and the mixture was allowed to warm to room temperature and stir for an additional $18 \mathrm{~h}$. The precipitate was removed by filtration and the crude product was purified by flash chromatography on silica gel, eluting with $\mathrm{CH}_{2} \mathrm{Cl}_{2}$ to provide 2-cyanoacetoxyethyl methacrylate as clear oil $(9.0 \mathrm{~g}, 91 \%)$. Prior to use, the monomer was further purified by distillation, $90-92{ }^{\circ} \mathrm{C}$ at $0.3 \mathrm{mmHg} .{ }^{1} \mathrm{H}$ NMR $\left(\mathrm{CDCl}_{3}\right): \delta 6.11(\mathrm{~s}, \mathrm{CH}, 1 \mathrm{H}), 5.59(\mathrm{t}, \mathrm{CH}, 1 \mathrm{H}), 4.43\left(\mathrm{~m}, \mathrm{CH}_{2} \mathrm{O}, 2 \mathrm{H}\right), 4.36\left(\mathrm{~m}, \mathrm{CH}_{2} \mathrm{O}, 2 \mathrm{H}\right)$, $3.49\left(\mathrm{~s}, \mathrm{CH}_{2} \mathrm{CN}, 2 \mathrm{H}\right), 1.92\left(\mathrm{~s}, \mathrm{CH}_{3}, 3 \mathrm{H}\right) ;{ }^{13} \mathrm{C} \mathrm{NMR}\left(\mathrm{CDCl}_{3}\right): \delta 166.2,162.9,135.2,125.5$, 112.9, 63.6, 61.3, 23.9, 17.4; IR $\left(\mathrm{NaCl}, \mathrm{cm}^{-1}\right): 2964,2264(\mathrm{C} \equiv \mathrm{N}), 1754(\mathrm{C}=\mathrm{O}), 1718$ $(\mathrm{C}=\mathrm{O}), 1637,1170$.

\section{A typical synthesis of Copolymer A1-1}

A solution of 2-cyanoacetoxyethyl methacrylate $(2.50 \mathrm{~g}, 12.7 \mathrm{mmol})$, methyl methacrylate $(4.0 \mathrm{~g}, 40 \mathrm{mmol})$, and AIBN $(32.8 \mathrm{mg}, 1.0 \mathrm{~mol} \%)$ in dry toluene $(14 \mathrm{~mL})$ was heated under nitrogen at $65-70^{\circ} \mathrm{C}$ for $20 \mathrm{~h}$ to provide a viscous solution. Solution was diluted with $\mathrm{CH}_{2} \mathrm{Cl}_{2}$ and precipitated twice into methanol to afford the copolymer as a white powder, and dried in a vacuum oven $(1 \mathrm{mmHg})$ at $60{ }^{\circ} \mathrm{C}$ overnight $(87 \%) .{ }^{1} \mathrm{H}$ NMR (Acetone- $\left.\mathrm{d}_{6}\right): \delta 4.5\left(\mathrm{CH}_{2} \mathrm{O}\right), 4.3\left(\mathrm{CH}_{2} \mathrm{O}\right), 3.9\left(\mathrm{CH}_{2}\right), 3.6\left(\mathrm{CH}_{3} \mathrm{O}\right), 1.8-2.1\left(\mathrm{CH}_{2}\right)$, 0.8-1.1( $\left(\mathrm{CH}_{3}\right) ;{ }^{13} \mathrm{C}$ NMR (Acetone- $\left.\mathrm{d}_{6}\right): \delta 177.8-178.4,164.8,114.7,64.2,63.3,53.0-55.1$, 52.0, 45.2-45.7, 24.9, 19.4, 17.4; IR $\left(\mathrm{NaCl}, \mathrm{cm}^{-1}\right) 2264(\mathrm{C} \equiv \mathrm{N}), 1756(\mathrm{C}=\mathrm{O}), 1729(\mathrm{C}=\mathrm{O})$, 1189, 1150; $[\eta]_{\text {inh }}=0.22 \mathrm{dL} / \mathrm{g}($ DMAc, $\mathrm{c}=0.5 \mathrm{~g} / \mathrm{dL}) ; \mathrm{T}_{\mathrm{g}}=83{ }^{\circ} \mathrm{C}(\mathrm{DSC}) ; \mathrm{T}_{\mathrm{d}}=220^{\circ} \mathrm{C}$ (TGA, nitrogen).

\section{A typical synthesis of NLO polymers NA1-a}


Under a anhydrous and oxygen-free condition, to a $50 \mathrm{~mL}$ round-bottomed flask were added copolymer A1-1(1.0 g, $7.6 \mathrm{mmol})$, sodium hydride $(0.016 \mathrm{~g}, 60 \%, 0.40 \mathrm{mmol})$ and compound $4(0.1 \mathrm{~g}, 0.2 \mathrm{mmol})$ in $10 \mathrm{~mL}$ of $\mathrm{THF}$ at $0^{\circ} \mathrm{C}$. Then the reaction was warmed to room temperature. After $6 \mathrm{~h}$, purification of the crude product by flash chromatography on silica gel, eluting with THF to remove the insoluble inorganic salt, and the filtrate solution was concentrated under reduced pressure then precipitated twice into methanol to afford FDCN chromophore - copolymer as a red powder (77\%).

\section{Synthesis of A2}

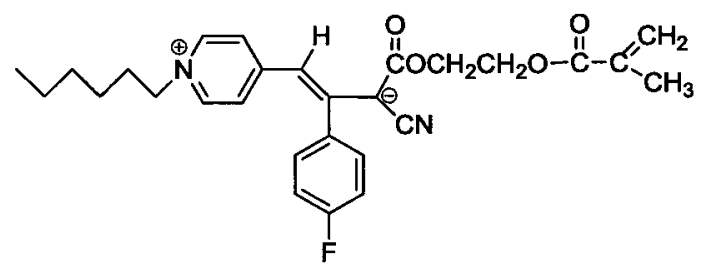

A Schlenk flask was charged with THF $(23 \mathrm{~mL})$, methacryloyl chloride $(0.53 \mathrm{~mL}$, $5.4 \mathrm{mmol}), \mathrm{FDCNOH}(1.86 \mathrm{~g}, 4.0 \mathrm{mmol})$, and $\mathrm{Et}_{3} \mathrm{~N}(0.76 \mathrm{~mL}, 5.4 \mathrm{mmol})$ and the mixture allowed to react with stirring at ambient temperature for $6 \mathrm{~h}$. The reaction mixture was diluted with $200 \mathrm{~mL}$ of ether and the organic layer was washed with water $(2 \times 200 \mathrm{~mL})$ and brine $(200 \mathrm{~mL})$ and then dried over $\mathrm{K}_{2} \mathrm{CO}_{3}$. The solvent was removed under reduced pressure, and the crude product was purified by an alumina column, eluting with $\mathrm{CH}_{2} \mathrm{Cl}_{2}$. The red band was collected, and the solvent was removed to afford pure $\mathbf{A 2}(1.52 \mathrm{~g}, 70 \%)$ without NMR measurement. IR: $2177(\mathrm{C} \equiv N), 1739(\mathrm{C}=\mathrm{O}), 1672(\mathrm{C}=\mathrm{C}), 1637(\mathrm{C}=\mathrm{O})$.

\section{A typical synthesis of NLO polymers NA2a}

A two-neck round bottom flask was charged with A2 (0.105 g, $0.220 \mathrm{mmol})$, $\operatorname{MMA}(0.450 \mathrm{~mL}, 4.18 \mathrm{mmol}), \operatorname{AIBN}(3.0 \mathrm{mg})$ and $1 \mathrm{~mL}$ of dry benzene. The reaction was heated to $80{ }^{\circ} \mathrm{C}$ under nitrogen for $18 \mathrm{~h}$. Solution was diluted with benzene and 
precipitated twice into methanol to afford the red NLO polymers NA2a (89\%).

${ }^{1} \mathrm{H}$ NMR $\left(\mathrm{CDCl}_{3}\right): \delta 8.5,8.2,7.5,7.0,4.3\left(\mathrm{CH}_{2} \mathrm{O}\right), 3.6\left(\mathrm{CH}_{3} \mathrm{O}\right), 1.3-1.5\left(\mathrm{CH}_{2}\right), 0.8-$ 1.1( $\left.\mathrm{CH}_{3}\right)$; IR $\left(\mathrm{KBr}, \mathrm{cm}^{-1}\right): 2177(\mathrm{C} \equiv \mathrm{N}), 1756(\mathrm{C}=\mathrm{O}), 1731(\mathrm{C}=\mathrm{O}), 1637,1485,1150$; UV-Vis: $\lambda_{\max }=502 \mathrm{~nm}(\mathrm{DMF}), 558 \mathrm{~nm}\left(\mathrm{CHCl}_{3}\right)$.

\section{A typical synthesis of host polyimide PI-1 $\mathbf{b}^{12}$}

To a solution of DAPBA (1.05 $\mathrm{g}, 3.12 \mathrm{mmol})$ in anhydrous DMAc $(13 \mathrm{~mL})$ was added 4,4'-(4,4'-isopropylidene diphenoxy)bis(phthalic anhydride) (1.60 g, $3.06 \mathrm{mmol})$ and phthalic anhydride $(0.0185 \mathrm{~g}, 0.125 \mathrm{mmol})$. The resulting solution was stirred at room temperature for $6 \mathrm{~h}$ and then acetic anhydride $(1.6 \mathrm{~mL})$ and pyridine $(0.8 \mathrm{~mL})$ were added. The reaction solution was stirred at room temperature for another $10 \mathrm{~h}$ and at 80 ${ }^{\circ} \mathrm{C}$ for $1 \mathrm{~h}$. To the viscous solution was added slowly acetic acid $(2 \mathrm{~mL})$. After the resulting precipitate was dissolved at $60{ }^{\circ} \mathrm{C}$ with stirring, the polymer solution was poured slowly into methanol $(200 \mathrm{~mL})$. The precipitated white polymer product was collected by filtration, washed thoroughly with hot methanol, and dried in a vacuum (1 $\mathrm{mmHg})$ oven at $120^{\circ} \mathrm{C}$ overnight: $2.4 \mathrm{~g}\left(95 \%\right.$ yield); $[\eta]_{\text {inh }}=0.33 \mathrm{dL} / \mathrm{g}(\mathrm{DMAc}, \mathrm{c}=0.5$ $\mathrm{g} / \mathrm{dL}) ; \mathrm{T}_{\mathrm{g}}=217{ }^{\circ} \mathrm{C}(\mathrm{DSC}) ; \mathrm{T}_{\mathrm{d}}=450{ }^{\circ} \mathrm{C}$ (TGA, nitrogen); ${ }^{1} \mathrm{H}$ NMR (300 MHz, DMSO$\left.d_{6}\right): \delta 13.42(\mathrm{~s}), 7.98(\mathrm{~d}, J=2.4 \mathrm{~Hz}), 7.52(\mathrm{~d}, J=7.2 \mathrm{~Hz}), 7.29-7.42(\mathrm{~m}), 7.11-7.21(\mathrm{~m})$, $1.75(\mathrm{~s}) ; \mathrm{IR}\left(\mathrm{KBr}, \mathrm{cm}^{-1}\right): 1778,1719\left(\mathrm{v}_{\mathrm{C}=\mathrm{O}}\right.$ of imide and carboxylic acid).

\section{Synthesis of NPI-a}

To an ice-cooled solution of PI-1b (0.204 g, $0.250 \mathrm{mmol})$, PpQDM-C3OH $(0.0260 \mathrm{~g}, 0.0625 \mathrm{mmol})$ and DMAP $(0.0335 \mathrm{~g}, 0.275 \mathrm{mmol})$ in anhydrous DMAc $(4 \mathrm{~mL})$ was added EDC (0.0575 $\mathrm{g}, 0.30 \mathrm{mmol})$. The solution was stirred at room temperature for 
$24 \mathrm{~h}$, then $0.1 \mathrm{~mL}$ of methanol (excess), DMAP $(0.067 \mathrm{~g}, 0.55 \mathrm{mmol})$ and EDC $(0.115 \mathrm{~g}$, $0.60 \mathrm{mmol}$ ) were added to the mixture and the reaction continued for another $24 \mathrm{~h}$ at room temperature. The reaction solution was added dropwise slowly into methanol (250 $\mathrm{mL}$ ) to precipitate the blue polymer. The blue polymer was re-dissolved into DMAc (4 $\mathrm{mL})$ and then precipitated into methanol $(200 \mathrm{~mL})$. The blue polymer NPI-a was collected by filtration and dried at $100{ }^{\circ} \mathrm{C}$ under vacuum: $0.1 \mathrm{~g}$ (85\% yield); PpQDM: $2.62 \mathrm{wt} \%$ in the polymer; ${ }^{1} \mathrm{H}$ NMR (300 MHz, DMSO- $d_{6}$ ): $\delta 10.45$ (br s), 9.09 (br m), 8.70 (br m), 8.20 (br m), 8.09 (br m), 7.95 (br m), 7.88 (br s), 7.78 (br s), 7.68 (br m), 7.45-7.08 (br m), 6.93 (br m), 4.66 (br s), 3.73 (br s), 1.70 (br s); IR (KBr, $\mathrm{cm}^{-1}$ ): 2178 and $2144\left(v_{\mathrm{C}=\mathrm{N}}\right.$ of cyano groups), 1777 and $1723\left(\mathrm{v}_{\mathrm{C}=\mathrm{O}}\right.$ of imide); $\mathrm{T}_{\mathrm{g}}=223{ }^{\circ} \mathrm{C}(\mathrm{DSC}) ; \mathrm{T}_{\mathrm{d}}$ $=265^{\circ} \mathrm{C}$ (TGA, nitrogen); UV-Vis: $\lambda_{\max }=702 \mathrm{~nm}(\mathrm{DMF})$.

NPI-b: 90\% yield; PpQDM: 4.71 wt $\%$ in the polymer; ${ }^{1} \mathrm{H}$ NMR (300 MHz, DMSO- $\left.d_{6}\right)$ : $\delta 10.49$ (br s), 9.07 (br s), 8.70 (br m), 8.74 (br m), 8.52 (br m), 8.38 (br m),8.29 (br m), 7.93 (br s), 7.78 (br m), 7.73 ( br s), 7.46-7.11 (br m), 6.88 (br m), 4.68 (br m), 4.22 (br m), 3.60(br m),1.70 (br s), 1.41(br m), 1.24 (br m), 0.88 (br m); IR (KBr, cm ${ }^{-1}$ ): 2177 and $2142\left(\mathrm{v}_{\mathrm{C}=\mathrm{N}}\right.$ of cyano groups), 1776 and $1722\left(\mathrm{v}_{\mathrm{C}=\mathrm{O}}\right.$ of imide); $\mathrm{T}_{\mathrm{g}}=185^{\circ} \mathrm{C}(\mathrm{DSC}) ; \mathrm{T}_{\mathrm{d}}$ $=264{ }^{\circ} \mathrm{C}\left(\mathrm{TGA}\right.$, nitrogen); UV-Vis: $\lambda_{\max }=695 \mathrm{~nm}(\mathrm{DMF})$.

\section{Synthesis of NPI-c to NPI-e (with an example of NPI-e)}

To an ice-cooled solution of PI-1b (0.204 g, $0.25 \mathrm{mmol})$, PpQDM-C3OH (0.031 $\mathrm{g}, 0.075 \mathrm{mmol})$, and DMAP $(0.040 \mathrm{~g}, 0.33 \mathrm{mmol})$ in anhydrous DMAc $(5 \mathrm{~mL})$ was added EDC $(0.0635 \mathrm{~g}, 0.33 \mathrm{mmol})$. After the solution was stirred at room temperature for $24 \mathrm{~h}$, BCBO (0.00167 g, $0.0125 \mathrm{mmol})$ and additional DMAP (0.045 g, $0.23 \mathrm{mmol})$ and EDC 
$(0.0635 \mathrm{~g}, 0.33 \mathrm{mmol})$ were added to the mixture and the mixture was stirring at room temperature for $24 \mathrm{~h}$; then $0.1 \mathrm{~mL}$ of 1 -hexanol (excess), DMAP $(0.045 \mathrm{~g}, 0.23 \mathrm{mmol})$ and EDC $(0.0635 \mathrm{~g}, 0.33 \mathrm{mmol})$ were added and the reaction continued for another $24 \mathrm{~h}$ at room temperature. The reaction solution was added dropwise slowly into methanol $(250 \mathrm{~mL})$ to precipitate the blue polymer. The blue polymer was re-dissolved into DMAc $(4 \mathrm{~mL})$ and then precipitated into methanol $(200 \mathrm{~mL})$. After the product was dried at 100 ${ }^{\circ} \mathrm{C}$ under vacuum, a blue polymer NPI-e was obtained : $0.12 \mathrm{~g}$ (85\% yield); PpQDM: $13.75 \mathrm{wt} \%$ in the polymer; ${ }^{1} \mathrm{H}$ NMR (300 MHz, DMSO- $\left.d_{6}\right): \delta$ (TMS, ppm) 10.49 (br s), 9.07 (br m), 8.50 (br m), 8.38 (br m), 8.28 (br m), 7.92 (br s), 7.76 (br m), 7.73 (br m), 7.65-7.10 (br m), 6.83 (br m), 4.72 (br m), 4.21 (br m), 3.78 (br m), 3.59 (br m), 1.70 (br s), 1.23 (br m), 1.06 (br m), $0.88(\mathrm{br} \mathrm{m}) ; \mathrm{IR}\left(\mathrm{KBr}, \mathrm{cm}^{-1}\right): 2178$ and $2144\left(\mathrm{U}_{\mathrm{C} \equiv \mathrm{N}}\right.$ of cyano groups), 1777 and 1723 ( $\mathrm{U}_{\mathrm{C}=\mathrm{O}}$ of imide); $\mathrm{T}_{\mathrm{g}}=180^{\circ} \mathrm{C}(\mathrm{DSC}) ; \mathrm{T}_{\mathrm{d}}=253{ }^{\circ} \mathrm{C}$ (TGA, nitrogen); UV-Vis: $\lambda_{\max }=695 \mathrm{~nm}(\mathrm{DMF})$.

NPI-c: $85 \%$ yield; PpQDM: $7.12 \mathrm{wt} \%$ in the polymer; ${ }^{1} \mathrm{H}$ NMR $\left(300 \mathrm{MHz}, \mathrm{DMSO}-d_{6}\right)$ : $\delta 10.45$ (br s), 9.05 (br m), 8.52 (br m), 8.38 (br m), 8.37 (br m), 7.92 (br s), 7.77 (br m), 7.72 (br m), 7.45-7.10 (br m), 6.87 (br m), 4.68 (br m), 4.22 (br m), 3.78 (br s), 3.60 (br m), 1.70 (br s), 0.85 (br m) ; IR (KBr, $\left.\mathrm{cm}^{-1}\right): 2178$ and 2142 ( $\mathrm{v}_{\mathrm{C} \equiv \mathrm{N}}$ of cyano groups), 1773 and 1733 ( $\mathrm{U}_{\mathrm{C}=\mathrm{O}}$ of imide); $\mathrm{T}_{\mathrm{g}}=200{ }^{\circ} \mathrm{C}(\mathrm{DSC}) ; \mathrm{T}_{\mathrm{d}}=260^{\circ} \mathrm{C}$ (TGA, nitrogen); UV-Vis: $\lambda_{\max }=695 \mathrm{~nm}(\mathrm{DMF})$.

NPI-d: $80 \%$ yield; PpQDM: 7.59 wt $\%$ in the polymer; ${ }^{1} \mathrm{H}$ NMR $\left(300 \mathrm{MHz}, \mathrm{DMSO}-d_{6}\right)$ : $\delta 10.49$ (br s), 9.07 (br m), 8.50 (br m), 8.38 (br m), 8.28 (br m), 7.92 (br s), 7.76 (br m), 7.73 (br m), 7.65-7.10 (br m), 6.83 (br m), 4.72 (br m), 4.21 (br m), 3.78 (br m), 3.59 (br 
m), 1.70 (br s), 1.23 (br m), 1.06 (br m), 0.88 (br m); IR (KBr, cm ${ }^{-1}$ ): 2178 and 2144 ( $\mathrm{U}_{\mathrm{C} \equiv \mathrm{N}}$ of cyano groups), 1777 and $1723\left(\mathrm{U}_{\mathrm{C}=\mathrm{O}}\right.$ of imide); $\mathrm{T}_{\mathrm{g}}=163{ }^{\circ} \mathrm{C}(\mathrm{DSC}) ; \mathrm{T}_{\mathrm{d}}=255^{\circ} \mathrm{C}$ (TGA, nitrogen); UV-Vis: $\lambda_{\max }=694 \mathrm{~nm}(\mathrm{DMF})$.

\section{Determination of PpQDM Content}

For the accurate determination of PpQDM content in NLO polyimides, a concentration calibration equation was established by measuring the absorption $(A)$ of PpQDM-C3OH in DMF at six concentrations at $690 \mathrm{~nm}$. By plotting the absorption against the concentration and by fitting the data with a straight line, the linear relationship between absorption and PpQDM concentration was defined as per the Beer-Lambert law:

$$
\mathrm{A}=0.44318 \times C-0.00469
$$

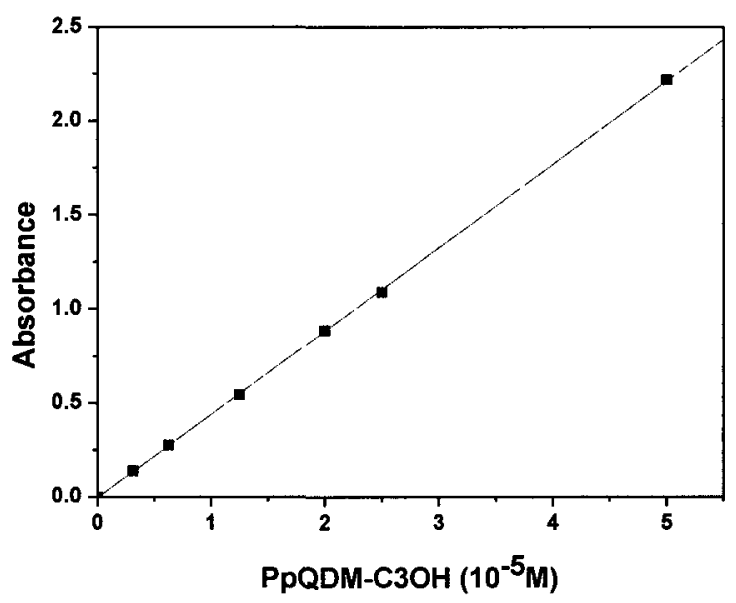

To determine the PpQDM-C3OH content in NLO polyimides, a polymer sample with known weight $(1.8 \mathrm{mg})$ was dissolved in a certain volume of DMF (10 mL) and the intensity of CT absorption is recorded, from which the weight of PpQDM is calculated and used to estimate the weight percentage of PpQDM-C3OH in NLO polyimides. 


\subsection{References}

(1) (a)Yesodha, S. K.; Sadashiva, C. K.; Pillaia, Tsutsumi, P. N. Prog. Polym. Sci. 2004, 29, 45. (b)Eaton, D. F. Science 1991, 253, 281. (c) Burland, D. M.; Miller,

R. D.; Walsh, C. A. Chem. Rev. 1994, 94, 31. (d) Dalton, L. R.; Harper, A. W.; Ghosn, R.; Steier, W. H.; Ziari, M.; Fetterman, H.; Shi, Y.; Mustacich, R. V.; Jen, A. K.-Y.; Shea, K. J. Chem. Mater. 1995, 7, 1060.

(2) (a)Briers, D.; Koeckelberghs, G.; Picard, I.; Verbiest, T.; Persoons, A.; Samyn, C. Macromol. Rapid Commun. 2003, 24, 841. (b) Lee, S. K.; Cho, M. J.; Jin, J.; Choi, D. H. J. Polym. Sci. A: Polym. Chem. 2007, 45, 531. (c)Scarpaci, A.; Cabanetos, C.; Blart, E.; Montembault, V.; Rodriguez, O. F. J. Polym. Sci. A: Polym. Chem. $2009,47,5652$.

(3) (a)Shi, Y.; Zhang, C.; Zhang, H.; Bechtel, H.; Dalton, L. R.; Robinson, B. H.; Steier, W. H. Science 2000, 288, 119. (b) Macchi, R.; Cariati, E.; Marinotto, D.; Roberto, D.; Tordin, E.; Ugo, R.; Bozio, R.; Cozzuol, M.; Pedron, D.; Mattei, G. J. Mater. Chem. 2010, 20, 1885. (c) Kajzar, F.; Krupka, O.; Pawlik, G.; Mitus, A.; Rau, I. Mol. Cryst. Liq. Cryst. 2010, 522, 180.

(4) Cheng, Y. J.; Luo, J. D.; Hau, S.; Bale, D. H.; Kim, T. D.; Shi, Z.; Tucker, N. M.; Tian, Y.; Dalton, L. R.; Reid, P. J.; Jen, A. K.-Y. Chem.Mater.2007, 19, 1154.

(5) Zhang, C.; Wang, C.; Dalton, L.R.; Zhang, H.; Steier, W. H. Macromolecules 2001, 34, 253.

(6) (a)Liang, Z.; Dalton, L. R.; Garner, S. M.; Kalluri, S.; Chen, A.; Steier, W. H. Chem. Mater. 1995, 7, 941. (b)Song, N. H. ; Men, L.; Gao, J. P.; Bai, Y.; Beaudin, A. M. R.; Yu, G.; Wang, Z. Y. Chem. Mater. 2004, 16, 3708. (c) Song, N. H. ; Men, L.; Gao, J. P.; Yu, G.; Beaudin, A. M. R.; Wang, Z. Y. J. Nonlinear Opt. Phys. Mater. 2005, 14, 367.

(7) (a)Garner, S. M.; Cites, J. S.; He, M.; Wang, J. Appl. Phys. Lett. 2004, 84, 1049. (b)Lu, Z.; Li, J.; Hua, J.; Li, X.; Qin, J.; Qin, A.; Ye, C. Syn. Met. 2005, 152, 217. (c)Bai, Y. W.; Song, N. H.; Gao, J. P.; Sun, X.; Wang, X.; Yu, G.; Wang, Z. Y. J. Am. Chem. Soc. 2005, 127, 2060. (d)Xiong, Y.; Tang, H.; Zhang, J.; Wang, Z. Y.; Campo, J.; Wenseleers, W.; Goovaerts, E. Chem. Mater. 2008, 20, 7465. 
(8) Kalluri, S.; Shi, Y.; Steier, W. H.; Yang, Z.; Xu, C.; Wu, B.; Dalton, L. R.; Appl. Phys. Lett. 1994, 65, 2651.

(9) Kang, H.; Facchetti, A.; Jiang, H.; Cariati, E.; Righetto, S.; Ugo, R.; Zuccaccia, C.; Macchioni, A.; Stern, C. L.; Liu, Z.; Ho, S.-T.; Brown, E. C.; Mark A. Ratner, M. A.; Marks, T. J. J. Am. Chem. Soc. 2007, 129, 3267.

(10) (a)Jin, D.; Londergan, T.; Huang, D. 2004, 4, 44. (b) Drummond, J. P.; Clarson, S. J. ; Zetts, J. S.; Hopkins, F. K.; Caracci, S. J. Appl. Phys. Lett. 1999, 74, 18.

(11) Wurthner, F.; Yao, S.; Debaerdemaeker, T.; Wortmann, R. J. Am. Chem. Soc. 2002, 124, 9431.

(12) Naiheng Song: "Development of Zwitterinic Nonlinear Optical Polyimides for Electro-Optic Applications", Ph. D. Thesis, Carleton University 2004.

(13) Mao, S. S. H.; Ra, Y.; Guo, L.; Zhang, C.; Dalton, L. R.; Chen, A.; Garner, S. M.; Steier, W. H. Chem. Mater. 1998, 10, 146.

(14) (a)Iacono, S. T.; Budy, S. M.; Jin, J.; Smith, D. W. J. Polym. Sci., Part 1: Polym. Chem. 2007, 45, 5706. (b)Budy, S. M.; Suresh, S.; Spraul, B. K.; Smith, D. W. J. Phys. Chem. C 2008, 112, 8089. (c)Luo, J. D.; Haller, M.; Li, H.; Kim, T.-D.; Jen, A. K.-Y. Adv. Mater. 2003, 15, 1635.

(15) (a)Sullivan, P. A.; Olbricht, B. C.; Akelaitis, A. J. P.; Mistry, A. A.; Liao, Y.; Dalton, L. R. J. Mater. Chem. 2007, 17, 2899. (b)Shi, Z.; Luo, J.; Huang, S.; Zhou, X. H.; Kim, T.-D.; Cheng, Y.-J.; Polishak, B. M.; Younkin, T. R.; Block, B. A.; Jen, A. K.-Y. Chem. Mater. 2008, 20, 6372. (c)Shi, Z.; Luo, J.; Hunag, S.; Cheng, Y.; Kim, T.; Polishak, B. M.; Zhou, X.; Tian, Y.; Jang, S.; Knorr, D. B., Jr.; Overney, R. M.; Younkin, T. R.; Jen, A. K.-Y. Macromolecules 2009, 42, 2438.

(16) (a)Wang, Z. Y. U.S. Patent 5,869,693. (b)Wang, Z. Y.; Suzzarini, L.; Gao, J. P. Tetrahedron Lett. 1997, 38, 5745. (c)Wang, Z. Y.; Kuang, L.; Meng, X. S.; Gao, J. P. Macromolecules 1998, 31, 5556.

(17) (a)Williams, A.; Ibrahim, I. T. Chem. Rev. 1981, 81, 589. (b)Hegarty, A. F.; McCormack, M. T.; Ferguson, G.; Roberts, P. J. J. Am. Chem. Soc. 1977, 99, 2016.

(18) Ying Xiong "Development of Zwitterinic Chromophores for Electro-Optic Applications", Ph. D. Thesis, Carleton University 2008. 


\section{Chapter 4 Electro-Optic Properties of Nonlinear Optical Materials}

\subsection{Introduction}

In NLO materials, the macroscopic electro-optic effect in terms of EO coefficient $\left(r_{33}\right)$ is determined by the number density of the embedded NLO chromophores, their hyperpolarizability, and their degree of noncentrosymmetrical orientational order.

A number of techniques have been used to create the required noncentrosymmetry in EO polymers including photo-assisted poling, ${ }^{1 \mathrm{a}}$ photothermal poling, ${ }^{1 \mathrm{~b}}$ and all optical poling, ${ }^{1 c}$ etc. However, the more common methods typically used are corona and parallel electrode poling.

Corona poling is advantageous because of its simplicity and the fast charging process as shown in Figure 4.1 (left). In corona poling, a sharp needle or wire is suspended above a polymer film and charged to several kilovolts to ionize the surrounding atmosphere and deposit charges on the surface of the films. Positive or negative charges can be deposited depending on the choice of electrode. Although high poling fields can be achieved in this way, the resulting strength can lead to dielectric damage to the polymer film surface resulting in optical losses after poling. ${ }^{\text {1d }}$

In the parallel electrode poling, the NLO polymer film was sandwiched between two electrodes (Figure 4.1, right), then the film subjected to heating up to the glass transition temperature while the poling field is applied. ${ }^{\text {le }}$ This method is advantageous to control the poling conditions, (such as voltage and the electric current monitored) and no damage to the surface of polymer films. The drawback of this technique is that the poling voltage is limited due to potential for dielectric breakdown in the polymer film. This 
limitation can be alleviated by poling under vacuum, controlling polymer purity in synthesis and film forming.
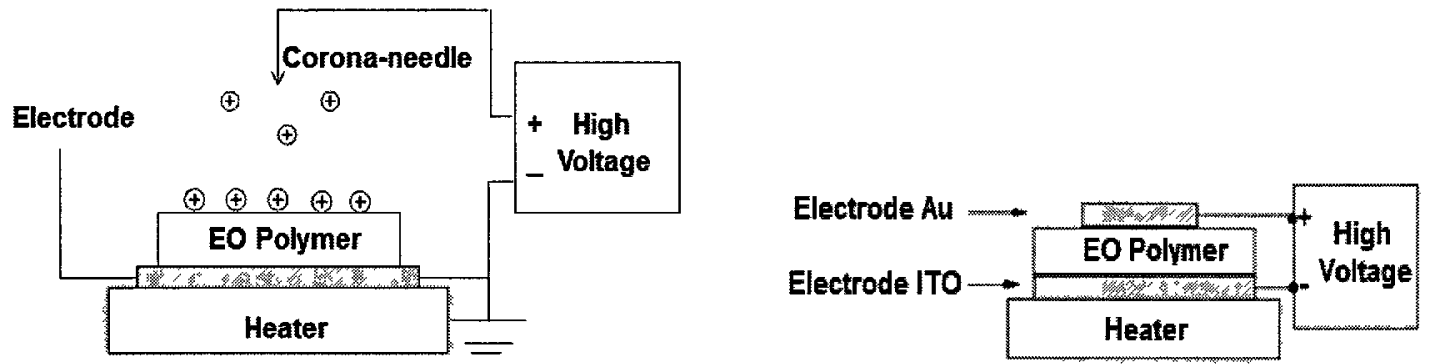

Figure 4.1. Schematic diagrams of the corona poling (left) and parallel electrode poling setup (right).

The net polar order of NLO materials in this research is achieved by electrode poling, since this process is commonly used in poling single-layer EO films to allow convenient characterization of NLO properties and screening of materials. The poling efficiency of NLO polymers is usually characterized as an order parameter of $<\cos ^{3} \theta>$. It depends on a number of factors, from both the material and technical aspects.

Usually, the maximum EO coefficient for a given polymer is achieved at the highest applicable poling field, often to a level where it nearly generates dielectric breakdown. Currently, medium electric field strengths $\left(20 \mathrm{~V} / \mu \mathrm{m}<\mathrm{E}_{\mathrm{POL}}<100 \mathrm{~V} / \mu \mathrm{m}\right)$ are widely used in the poling process, ${ }^{2}$ electrode-poling voltages of around $100 \mathrm{~V}$ per micrometer are achievable for highly efficient NLO polymers. ${ }^{3}$ Dalton and co-workers ${ }^{4}$ systematically investigated the factors affecting the order parameter of poled polymers, showing that intermolecular electrostatic interactions associated with dipole moment, shape, and concentration of chromophores play an important role in lowering the maximum number density and poling efficiency. 
Higher poling field usually produces higher EO coefficient. However, high poling voltage could induce local electrical discharges in low-density domains of the material or film defects (e.g., voids and impurities), and lead to high leak through currents (LTCs) that cause physical damages to the poled films. ${ }^{5}$

One of the most critical aspects in the poling of polymers is to obtain the maximum possible electric field without dielectric breakdown. Therefore, considerable research efforts have been spent on improving high field poling of NLO polymers. An effective approach to improve the poling of EO polymers is to insert a thin buffer layer of spin-on materials as a barrier between the electrode and the EO polymer layer to form a double-layer structure between the two poling electrodes (Figure 4.2 ). ${ }^{6}$ Compared to a single-layer NLO film having intimate contact with two electrodes, the presence of an additional layer can delay the onset of dielectric breakdown, and effectively enhance the strength of the maximum applicable poling fields.

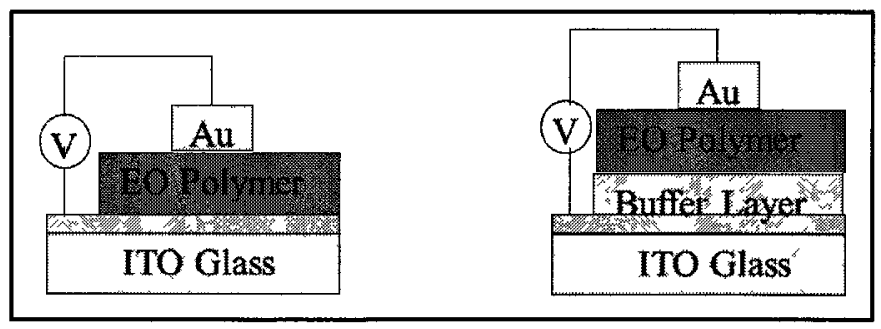

Figure 4.2. Sandwiched single-layer (left) and double-layer structures (right) for the poling of thin film EO materials.

The selection of buffer layer materials includes inorganic SiOx networks, ${ }^{7}$ conducting polymer blends of poly(ethylene dioxythiophene):poly(styrenesulfonic acid) (PEDOT:PSS) with poly(vinyl alcohol)(PVA), ${ }^{8}$ and sol-gel derived $\mathrm{TiO}_{2} \cdot{ }^{9}$ It has been demonstrated that the application of a suitable barrier layer enhanced the $r_{33}$ by $15-100 \%$ 
on conventional NLO polymers, such as PMMA/DR1 and doped APC systems, compared to the results of poled single-layer films. ${ }^{5,7,8,9}$ For NLO polymers based on zwitterionic PQDM and PeQDM, strong inter-chromophore electrostatic interactions were found due to the very large dipole moments of those chromophores. PpQDM doped polymer systems with relatively high dielectric constants (e.g., in PVP) may attenuate the fields associated with intermolecular electrostatic interactions and alleviate the problem in achieving a high poling efficiency. The inter-chromophore electrostatic interactions effect regarding the FDCN-based NLO polymers are unclear. Barrier materials applied into making double layer structure device of zwitterionic chromophores have not been studied before, especially for the PpQDM-based NLO polyimides with much higher concentration of zwitterionic NLO chromophores.

Therefore, this chapter describes the EO property measurements of 1) FDCN doped PES guest-host polymers; 2) FDCN-MMA NLO polymers (NA2); 3) PpQDM doped PVP guest-host polymers; 4) crosslinkable PpQDM NLO polyimides (Chapter 3). Detailed poling methods including conventional electrode poling and in situ poling and EO coefficient measurement method are introduced in the experimental section. The NLO chromophores loading and electrical properties of NLO polymer films are studied. The correlation studies of EO coefficients with the chromophore structures, poling conditions and NLO polymer film structures (e.g., single layer and double layer) will be also discussed. 


\subsection{Electro-Optic Properties of FDCN-Based NLO Polymers}

\subsubsection{Electro-Optic Properties of FDCN-PES Guest-Host Polymers}

As discussed in Chapter 3, PES represents a suitable host for FDCN guest-host system because of its high quality film-forming ability, film optical clarity, and high glass transition temperature of $225^{\circ} \mathrm{C}$, which could prevent or minimize the chromophore relaxation after poling. Therefore, guest-host FDCN NLO polymer films were prepared using FDCN chromophores and commercially available PES. FDCN chromophores and PES were dissolved in DMF to form 2 wt \% FDCN/PES solution and stirred overnight at room temperature in the dark. After the solution was filtered through a $0.2 \mu \mathrm{m}$ syringe filter, the filtered solution was cast onto ITO glass substrate $\left(2.56 \mathrm{~cm}^{2}\right)$ to produce films of about $1 \sim 3 \mu \mathrm{m}$ thickness and dried at $45^{\circ} \mathrm{C}$ overnight under the protection of nitrogen. The films were further dried in a vacuum oven overnight at $85^{\circ} \mathrm{C}$ to remove the residual solvent. A thin gold layer with a thickness of 100-150 nm was sputtered on the films. The ITO and gold layers were used as both poling and modulation electrodes.

FDCNs can be doped up to 10 wt \% into PES and smooth thin films can be prepared by solution casting. The loading density of FDCN is higher than that of PQDM chromophores in PES system, which was limited to about $2 \mathrm{wt} \%$, as larger concentrations led to phase separation, due to strong intermolecular interactions arising from the large dipole moments. ${ }^{10}$

The electrode poling process was carried out following a conventional way, under the protection of nitrogen in the dark in an electrode poling setup, the sample cells were gradually heated from room temperature to the poling temperature (e.g., $190{ }^{\circ} \mathrm{C}$ for 
FDCN/PES) at a heating rate of $5{ }^{\circ} \mathrm{C} / \mathrm{min}$, and a direct current (DC) voltage applied across the polymer films was maintained between 60 and $70 \mathrm{~V} / \mu \mathrm{m}$.

During the poling process, the electric current was monitored to optimize poling efficiency and to control local electrical breakdown in the films. The increment of current should be dominantly ascribed to the chromophore alignment under the external electric field and the chain rearrangement of polymer, which is mostly accomplished around the $\mathrm{T}_{\mathrm{g}}$ of the polymer. The monitoring of the current also enabled more efficient poling of the sample. Generally, the breakdown of the electric field through the polymeric film is accompanied by an abrupt overflow of the electric current. The current dropped slowly after it reached the maximum, and even under a constant poling field.

The poling temperature is about $5-10^{\circ} \mathrm{C}$ below the $\mathrm{T}_{\mathrm{g}}$ of the polymer films. After the films were held at the poling temperature for $20 \mathrm{~min}$, the heating was turned off while keeping the poling field. At the same time, the current declined due to the remarkable increase in resistance of the polymeric films. After reaching room temperature, the poling voltage was taken off and the EO coefficients of the poled polymers were measured immediately at $1550 \mathrm{~nm}$ using the simple reflection technique (See experimental section $4.5 .4) .^{11}$

All samples possess very little absorbance at the measured wavelength of 1550 nm. Thus, we did not consider the absorption induced resonance enhancement. The EO coefficient, $r_{33}$ was determined on a Teng-Man reflection setup. An alternate current (AC) voltage was applied to each sample to observe the modulated signal $\left(I_{\mathrm{m}}\right)$. The linear EO coefficient of the poled films was calculated by the following equation. The $r_{33}$ value is directly proportional to $I_{\mathrm{m}} / I_{\mathrm{c}}$ in eq $1 .{ }^{11 \mathrm{a}}$ 


$$
r_{33}=\frac{3 \lambda I_{m}}{4 \pi V_{m} I_{c} n^{2}} \frac{\left(n^{2}-\sin ^{2} \theta\right)^{3 / 2}}{\left(n^{2}-2 \sin ^{2} \theta\right)} \frac{1}{\sin ^{2} \theta}
$$

where $n$ is the refractive index of the polymer materials and $I_{\mathrm{m}}$ is the amplitude of EO modulation. $V_{\mathrm{m}}$ is the modulating voltage applied to the sample and $I_{\mathrm{c}}$ is the half intensity of incident light. The molecular properties of FDCN chromophores and EO properties of FDCN doped NLO polymers are summarized in Table 4.1.

Although the FDCN series based on the picolium donor and dicyano acceptor exhibits moderate values of $\mu \beta$ (See Table 4.1), compared to PQDM, which $\mu \beta=74,600$ $\times 10^{-48}$ esu, more than ten times larger than that of FDCN, FDCN/PES thin films display almost the same EO coefficients as PQDM/PES thin films (21 pm/V of $1 \mathrm{wt} \%$ in PES), ${ }^{10}$ due to its higher loading ability into PES (5 wt \%) than the PQDM chromophores.

Table 4.1. The molecular properties of FDCN chromophores and EO properties of FDCN doped PES polymers

\begin{tabular}{cccccccc}
\hline Chromophore & $\begin{array}{c}\mu \\
(\mathrm{D})\end{array}$ & $\begin{array}{c}\beta \\
\left(10^{-30} \mathrm{esu}\right)\end{array}$ & $\begin{array}{c}\mu \beta \\
\left(10^{-48} \mathrm{esu}\right)\end{array}$ & $\begin{array}{c}\mathrm{N} \\
(\text { Polymer })\end{array}$ & $\begin{array}{c}n \\
(1.5 \mu \mathrm{m})\end{array}$ & $\begin{array}{c}\mathrm{T}_{\mathrm{p}} \\
\left({ }^{\circ} \mathrm{C}\right)\end{array}$ & $\begin{array}{c}r_{33} \\
(\mathrm{pm} / \mathrm{V})\end{array}$ \\
\hline FDCN & 21.6 & -320 & -6720 & $5 \%$ & 1.6206 & 190 & 20 \\
FCNE & 11.4 & -495 & -5643 & $5 \%$ & 1.6204 & 180 & 18 \\
\hline
\end{tabular}

$\mathrm{N}$ : chromophore contents; $\mathrm{n}$ : refractive index at $1550 \mathrm{~nm} ; \mathrm{T}_{\mathrm{p}}$ : poling temperature

The results indicated that the aromatic group attached perpendicular to the main axis of the FDCN chromophore leads to a larger EO coefficient compared to its plain analogue molecule PQDM in the same polymer matrix. This is attributed to the ability of the bulky aromatic side chain to keep neighboring molecules away from each other and thus reduce their intermolecular interactions. In addition, it suggested that PES is a proper polymer matrix with proper polarity for less polar FDCN than for the more polar PQDM 
chromophore, because the EO coefficient is affected by different polymer densities and partially due to the influence of the polymer environment to the poling efficiency.

Compared with a neutral benchmark chromophore, namely CLD-1 $(\mu \beta=14,065 \times$ $\left.10^{-48} \mathrm{esu}, \mu=13\right),{ }^{12}$ which EO coefficients are as high as $16 \sim 22 \mathrm{pm} / \mathrm{V}$ at $1550 \mathrm{~nm}$ for a 15 wt \% chromophore loading in PMMA. ${ }^{13}$ The FDCN series has relatively lower hyperpolarizabilities, but displays the same level of $r_{33}$ with CLD-1 contributing to their large $\mu$ values, which is the significant zwitterionic molecular nature.

\subsubsection{Electro-Optic Properties of FDCN-MMA NLO Polymers}

In order to reduce relaxation of the EO signal and increase the number density of active chromophores in NLO polymer systems, the FDCN chromophore was covalently incorporated into an FDCN-MMA polymer system (NA2), which were expected to have higher $r_{33}$ than that of the FDCN doped system.

For the poling studies, NA2 polymer films with thickness of 1-3 $\mu \mathrm{m}$ on ITO glass were prepared by solution casting directly. The films were dried at $60-80{ }^{\circ} \mathrm{C}$ under vacuum $(1 \mathrm{mmHg})$ for $24 \mathrm{~h}$ before sputtering a thin layer $(\sim 150 \mathrm{~nm})$ of gold as a top electrode onto the polymer films for electrode poling. In a general experiment, the sample was mounted on the poling stage and the temperature was increased in several minutes up to around $T_{\mathrm{g}}$. The poling field was then applied to the sample at a ramping rate of $10 \mathrm{~V} / \mathrm{min}$.

In comparison with FDCN doped PES system, NA2 polymer films showed a better stability to high electric fields. A field above $70 \mathrm{~V} / \mu \mathrm{m}$ was readily achieved for 
these polymers, and the NA2 polymers displayed large EO coefficients of 24 and 30 $\mathrm{pm} / \mathrm{V}$ for the 7 and $14 \mathrm{wt} \%$ polymers, respectively (Table 4.2 ).

Table 4.2. Characterization of FDCN-MMA polymers

\begin{tabular}{ccccccc}
\hline $\begin{array}{c}\text { NLO } \\
\text { polymer }\end{array}$ & $\begin{array}{c}\text { FDCN } \\
(\mathrm{wt} \%)\end{array}$ & $\begin{array}{c}\mathrm{T}_{\mathrm{g}} \\
\left({ }^{\circ} \mathrm{C}\right)\end{array}$ & $\begin{array}{c}\text { Film thickness } \\
(\mu \mathrm{m})\end{array}$ & $\begin{array}{c}\mathrm{n} \\
(1.5 \mu \mathrm{m})\end{array}$ & $\begin{array}{c}\mathrm{T}_{\mathrm{p}} \\
\left({ }^{\circ} \mathrm{C}\right)\end{array}$ & $\begin{array}{c}r_{33} \\
(\mathrm{pm} / \mathrm{V})\end{array}$ \\
\hline NA2a & 7 & 95 & 2.01 & 1.4972 & 90 & 24 \\
NA2b & 14 & 126 & 1.89 & 1.4970 & 120 & 30 \\
\hline
\end{tabular}

The maximal level of chromophore loading was achieved around $14 \mathrm{wt} \%$ without showing any phase separation in solution-cast films, indicating the good compatibility of FDCN with PMMA. A higher number density of NLO chromophore in bulk polymers is highly desired to impart a large microscopic optical nonlinearity in NLO chromophores to a large macroscopic EO coefficient in NLO polymer, however, the higher number density in polymers may lead to either phase separation eventually or attenuation of the poling efficiency due to the increased intermolecular electrostatic interactions. ${ }^{12}$ For example, the PQDM with a higher $\mu \beta$ value containing polyimides NLO polymers developed by our group, the optimal concentrations were below $11 \mathrm{wt} \%{ }^{13}$

As expected, the measured EO coefficient of the poled NA2 polymer films with higher chromophore content, such as $14 \mathrm{wt} \%$ is much higher than that of the FDCN doped PES system (5 wt \%) and reached values comparable to that of the bulk inorganic NLO single crystal lithium niobate $\left(\mathrm{LiNbO}_{3}, r_{33}=31 \mathrm{pm} / \mathrm{V}\right)$, which is currently used in the commercial EO modulators, and even higher than those of FTC/CLD-based NLO polymers with similar number densities. ${ }^{14,15}$ 


\subsection{Electro-Optic Properties of PpQDM-Based NLO Polymers}

\subsubsection{Electric Poling of PpQDM/PVP Guest-Host Polymers}

To investigate the potential of the PpQDM chromophores in EO applications, a doping study was carried out and the macroscopic EO activity of the poled NLO guesthost polymers was assessed. As discussed in Chapter 3, PVP was chosen in our study as a host polymer for PpQDMs based on the absorption study of PpQDM-Met doped systems, good film forming ability and higher loading density of the PpQDM chromophores. For comparison, the EO properties of PpQDM/PES guest-host NLO polymer thin films were also studied.

PpQDM-Met and PpQDM-Ben were firstly doped into PES and PVP matrix materials in a host-guest way in the solvent of DMF with the chromophore contents of 1 , 3 and 5 wt \%, respectively; then visually clear PpQDM-Met polymer and PpQDM-Ben polymer films were prepared (See experimental section). These films were of high quality and pinhole free, and did not exhibit phase separation. These features are important to ensure successful poling without dielectric breakdown and to produce reproducible EO coefficients.

Table 4.3. Characterization of PpQDM guest-host polymer thin films

\begin{tabular}{ccccc}
\hline Chromophore & $\begin{array}{c}\mathrm{N} \\
\text { (Polymer) }\end{array}$ & $\begin{array}{c}\mathrm{T}_{\mathrm{g}} \\
\left({ }^{\circ} \mathrm{C}\right)\end{array}$ & $\begin{array}{c}\mathrm{T}_{\mathrm{p}} \\
\left({ }^{\circ} \mathrm{C}\right)\end{array}$ & $\begin{array}{c}r_{33} \\
(\mathrm{pm} / \mathrm{V})\end{array}$ \\
\hline PpQDM-Met & 1\%(PES) & 210 & 170 & 6 \\
PpQDM-Met & $\mathbf{3 \% ( P V P )}$ & 165 & 140 & 16 \\
& & & & \\
PpQDM-Ben & $\mathbf{1 \% ( P E S )}$ & 210 & 170 & 12 \\
PpQDM-Ben & $5 \%(\mathrm{PES})$ & 210 & 170 & 25 \\
PpQDM-Ben & $\mathbf{5 \% ( P V P )}$ & 165 & 140 & 40 \\
\hline
\end{tabular}


A typical parallel electrode poling process and EO coefficient measurement was carried out as described in the 4.2 .1 section; Table 4.3 collects the results from characterization of both PpQDM-Met and PpQDM-Ben guest-host polymer thin films.

\subsubsection{Optimization of Poling Conditions}

\section{Poling Temperature}

Typically, the electric poling of NLO polymers is carried out around the $T_{g}$, as the segmental chain relaxation of the polymers at such a temperature allows for good chromophore mobility. However, $\mathrm{T}_{\mathrm{g}}$ is not the only factor governing the poling temperatures. Others like thermal stability and dielectric breakdown temperature under certain poling electric fields should also be considered.

To avoid degradation of the PpQDM chromophore during poling process, the thermal stability of the PpQDM-Ben/PVP thin films in nitrogen was evaluated by UV-Vis spectroscopy. As shown in Figure 4.3, when PpQDM-Ben/PVP thin film was heated at various temperatures below $120{ }^{\circ} \mathrm{C}$ for $30 \mathrm{~min}$ in nitrogen, the $\mathrm{CT}$ absorption band remained basically unchanged; when the film was heated at temperature above $140{ }^{\circ} \mathrm{C}$, a rapid decrease in the CT absorption was observed, a second peak at $490 \mathrm{~nm}$ started appearing and kept increasing to the maximum value, while the $\mathrm{CT}$ absorption of PpQDM-Ben chromophore completely disappeared and the color of the film changed from blue to light pink.

The color bleaching behavior of PpQDM is similar with that of PQDM. The decomposition of PQDM in solution and in the solid state (guest-host in PES) has been investigated by a former graduate student in our group. ${ }^{16}$ The results showed that PQDM chromophores are labile to the presence of oxygen, especially upon exposure to visible 
light and heating. The same may also explain the color fading for the PpQDM-Ben/PVP film at high temperatures. Therefore, the poling was performed at $170{ }^{\circ} \mathrm{C}$ for PpQDMs/PES and $140{ }^{\circ} \mathrm{C}$ for PpQDMs/PVP, respectively, which are about $10^{\circ} \mathrm{C}$ below the $\mathrm{T}_{\mathrm{g}}$ of the corresponding polymer films.

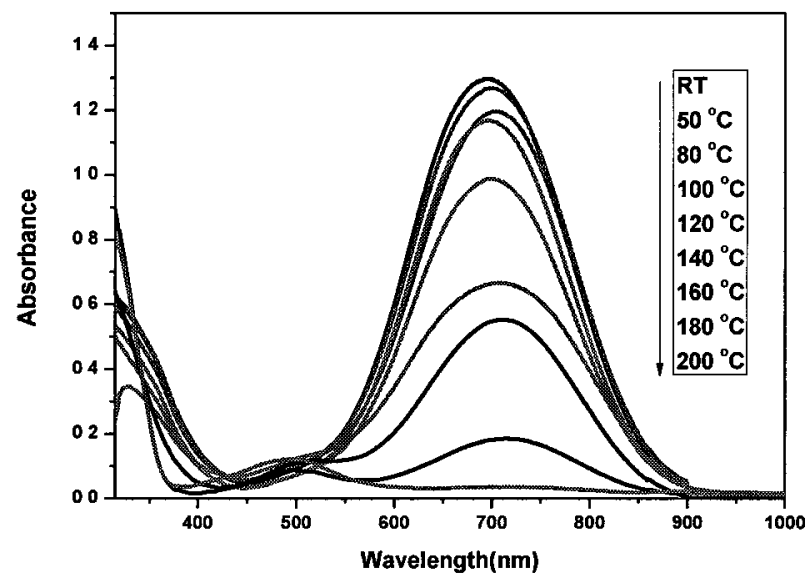

Figure 4.3. UV-Vis absorption spectra of thin film of PpQDM-Ben /PVP at elevated temperatures in nitrogen.

\section{Poling Voltage}

For the parallel electrode poling of NLO polymers, higher electric field is desired to afford a strong dipole-field interaction and a high order parameter. However, to simply pursue a high voltage is not appropriate, since dielectric breakdown may often occur. To obtain optimized electric field for poling, the EO coefficient of the sample was traced under various poling voltages. During the poling process, the electric current was monitored to optimize poling efficiency and to control local electrical breakdown in the films. It was found that a dielectric breakdown occurred at the high electric field of 80$100 \mathrm{~V} / \mu \mathrm{m}$, therefore relatively low electric fields, such as $\sim 70 \mathrm{~V} / \mu \mathrm{m}$ were utilized for the poling of the NLO polymers based on the various conductivity and achievable high 
fields of different quality of polymer films.

\section{Effect of Polymer Matrix}

PpQDM-Ben/PVP (5 wt \%) demonstrated the highest $r_{33}$ value $(\sim 40 \mathrm{pm} / \mathrm{V})$ among the tested guest-host NLO polymers, while PpQDM-Ben/PES, PpQDM-Met/PVP and PpQDM-Met/PES displayed lower $r_{33}$ values of around 25,16 and $6 \mathrm{pm} / \mathrm{V}$, respectively (Table 4.3). These results indicated that the intermolecular aggregation of the chromophores in the more polar PVP polymer matrix was effectively reduced compared to PES and the poling efficiency of the PVP system was enhanced due to the relatively polar environment. For example, PpQDM-Ben/PVP (5 wt \%) showed higher $r_{33}$ value $(\sim 40 \mathrm{pm} / \mathrm{V})$ than that of PpQDM-Ben/PES $(5 \mathrm{wt} \%, \sim 25 \mathrm{pm} / \mathrm{V})$ even though it was poled at a lower poling temperature $\left(140^{\circ} \mathrm{C}\right)$ compared with $170^{\circ} \mathrm{C}$ in PES.

\section{Effect of Chromophore Shape}

It is interesting to find that PpQDM-Ben and PpQDM-Met have almost same $\mu \beta$ value, but higher compatibility with polymer host and higher corresponding $r_{33}$ value were found in PpQDM-Ben than in PpQDM-Met. First, doping density of PpQDM-Ben in both PVP and PES can reach 5 wt \% and are higher than that of PpQDM-Met (3 wt \% and 1 wt \%, respectively); second, PpQDM-Ben/PES (5 wt \%) exhibited almost four fold higher $r_{33}$ value than that of PpQDM-Met/PES (1 wt \%) system due to the higher chromophore loading density. Even with the same amount of the chromophore inside, the $r_{33}$ value of the PpQDM-Ben/PES (1 wt \%) was two times higher than that of the PpQDM-Met/PES (1 wt \%) system. 
To investigate the difference of the chromophores' structure, the minimum energy conformations for three PpQDM-based chromophores such as PpQDM-Met, PpQDMBen, PpQDM-C3OH were determined by computer calculations using the MP6 method; in comparison, the minimum energy conformations of PQDM was also calculated. The optimized geometrical structures of these chromophores are illustrated in Figure 4.4.

(a)
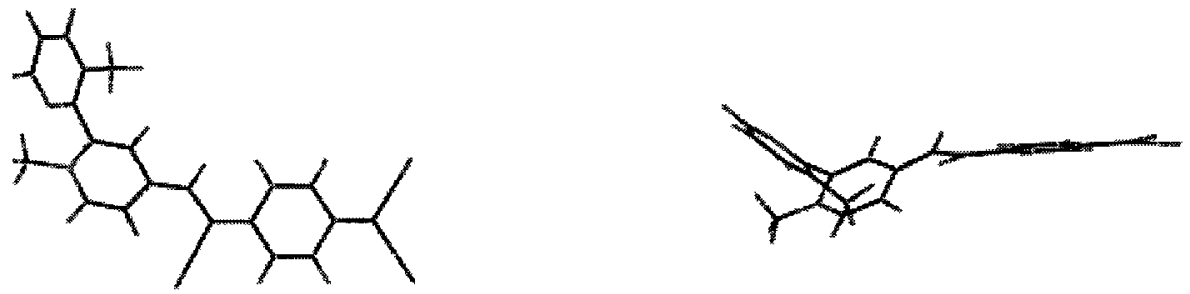

(b)
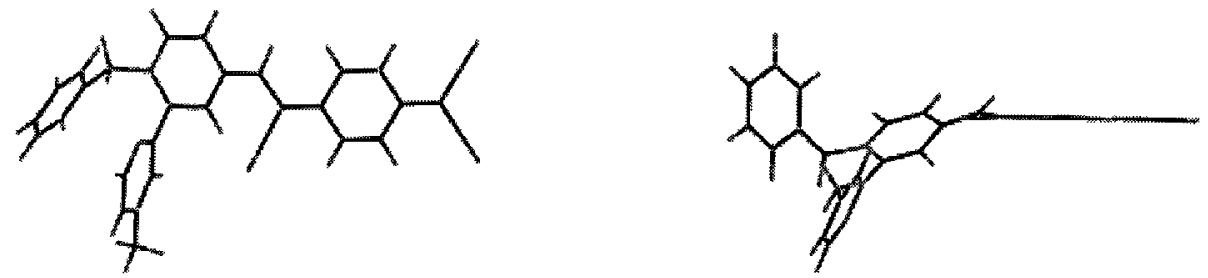

(c)
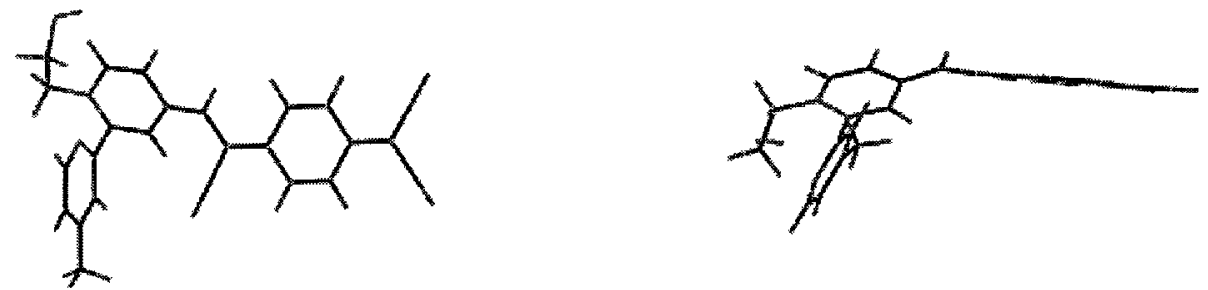

(d)
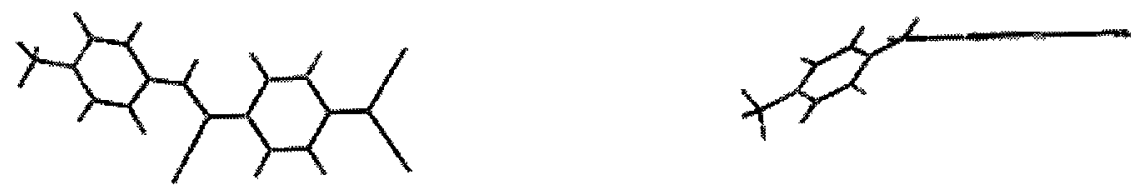

Figure 4.4. Geometries of chromophores: (a) PpQDM-Met (b) PpQDM-Ben (c) PpQDM-C3OH (d) PQDM-Met. 
Both PQDM and PpQDM chromophores have plane structure, PQDM is more rigid and rod-like than PpQDM. The methyl pyridine in the PpQDM chromophores were aligned almost perpendicular to the long molecular axis of the chromophores.

Compared with PpQDM-Met, the donor group of PpQDM-Ben is encompassed by an additional bulky benzyl group in the PpQDM-Ben, it's plane is out of the molecular main chain plane, therefore the chromophores are more difficult to be interacted electrostatically, so that they are more labile to be poled. It is indicated that the bulky donor size and suitable molecular shape of PpQDM-Ben can lead to large $r_{33}$ value.

For the practical application of NLO polymers, the long-term stability of the EO coefficient at elevated temperatures is important. The temporal stability of EO coefficient of the poled PpQDM-Ben/PVP film was investigated by measuring the $r_{33}\left[r_{33}(t) / r_{33}\left(t_{0}\right)\right]$ as a function of time at $80^{\circ} \mathrm{C}$ (operational temperature) in the dark. Figure 4.5 shows the temporal stability of EO coefficient for the poled PpQDM-Ben/PVP film. The $r_{33}$ value decreased around $10 \%$ of the initial value after the first $160 \mathrm{~h}$ in the dark and under nitrogen atmosphere. This large drop of the EO coefficient at the beginning of thermal treatment may arise as a result of the relaxation of the ordered state, i.e. from the recovery of bond angle and bond length of the oriented chromophores. ${ }^{17}$

The $r_{33}$ value maintains constant around $15 \%$ relaxation of the initial value after the second $160 \mathrm{~h}$ in air. The results show high stabilities of NLO responses at elevated temperatures, and PVP polymer matrix have proper polarity to hold the oriented chromophores, thus restricting the local movement of the chromophores and reducing orientation relaxation. 


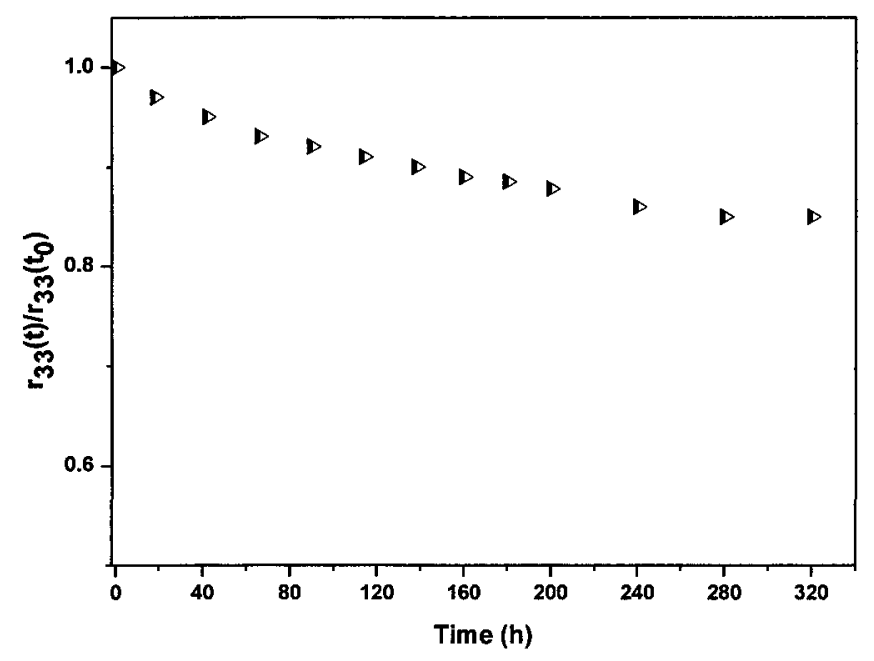

Figure 4.5. Temporal stability of the $r_{33}$ for the poled PpQDM-Ben/PVP at $80^{\circ} \mathrm{C}$.

\subsubsection{In situ Poling of PpQDM-PVP Guest-Host Polymers}

To explore the intrinsic EO properties of the PpQDM series chromophores, the in situ EO coefficient measurement of poled PpQDM-PVP guest-host materials at the laser wavelength of $1550 \mathrm{~nm}$ was performed by a modified Teng-Man reflection technique. ${ }^{18}$ In this measurement, $r_{33}$ signals were collected during the process of heating and cooling in the presence of a poling field. Overlaying a DC poling field with an $\mathrm{AC}$ voltage, real time monitoring of the ratio $I_{m} / I_{c}$ allowed direct evaluation of optimal $r_{33}$ values under the controlled experimental parameters such as temperature and applied voltage.

A representative in situ poling process using PpQDM-Ben doped PVP polymer system is shown in Figure 4.6. The loading concentration of the chromophore is $5 \mathrm{wt} \%$. In a general experiment, the sample was mounted on the poling stage and the temperature was increased in several minutes up to around $\mathrm{T}_{\mathrm{g}}$. The poling field was then applied to the sample at a ramping rate of $10 \mathrm{~V} / \mathrm{min}$. Since no electric current was monitored during our in situ poling process, the ramping of the poling field at $50{ }^{\circ} \mathrm{C}$ and $80{ }^{\circ} \mathrm{C}$ was 
recorded to investigate the maximum poling voltage (Table 4.4), a linear dependence of the $r_{33}$ values versus the poling voltages has been observed.

Table 4.4. EO coefficient (pm/V) of PpQDM-Ben/PVP (5 wt \%) under various temperature and poling fields

\begin{tabular}{cccccc}
\hline \multirow{2}{*}{$\begin{array}{c}\text { NLO } \\
\text { polymer }\end{array}$} & $\mathrm{T}_{\mathrm{p}}$ & \multicolumn{4}{c}{ Poling Field $(\mathrm{V} / \mu \mathrm{m})$} \\
\cline { 3 - 6 }$\left({ }^{\circ} \mathrm{C}\right)$ & & 35 & 50 & 60 & 70 \\
\hline PpQDM-Ben & 50 & 5.5 & 15.5 & 19.8 & 33.0 \\
PpQDM-Ben & 80 & 6.2 & 16.4 & 25.2 & 40.1 \\
\hline
\end{tabular}

Since the poled films show the largest $r_{33}$ values of 33 and $40 \mathrm{pm} / \mathrm{V}$ in the poling field around $70 \mathrm{~V} / \mu \mathrm{m}$ at $50^{\circ} \mathrm{C}$ and $80^{\circ} \mathrm{C}$, respectively, the ramping of the in situ poling field of PpQDM-Ben/PVP (5 wt \%) NLO polymers was ceased and maintained at 70 $\mathrm{V} / \mu \mathrm{m}$ to achieve excellent poling efficiency by considering the stability of the film. The highest $r_{33}$ values of the films PpQDM-Met and PpQDM-Ben were obtained with the chromophore concentrations of 3 and 5 wt $\%$, respectively. The resultant EO properties of PpQDM doped NLO polymers under the optimal poling condition are summarized in Table 4.5.

Table 4.5. Characterization of PpQDM Guest-host polymers under in situ poling

\begin{tabular}{cccccc}
\hline Chromophore & $\begin{array}{c}\mathrm{N} \\
(\text { Polymer })\end{array}$ & $\begin{array}{c}\mathrm{T}_{\mathrm{g}} \\
\left({ }^{\circ} \mathrm{C}\right)\end{array}$ & $\begin{array}{c}\mathrm{T}_{\mathrm{p}} \\
\left({ }^{\circ} \mathrm{C}\right)\end{array}$ & $\begin{array}{c}\mathrm{V}_{\mathrm{p}} \\
(\mathrm{V} / \mu \mathrm{m})\end{array}$ & $\begin{array}{c}r_{33} \\
(\mathrm{pm} / \mathrm{V})\end{array}$ \\
\hline PpQDM-Met & $\mathbf{3 \% ( P V P )}$ & 170 & 120 & 70 & 36 \\
PpQDM-Met & $\mathbf{3 \% ( P V P )}$ & 170 & 140 & 70 & 40 \\
PpQDM-Ben & $\mathbf{5 \% ( P V P )}$ & 170 & 50 & 70 & 33 \\
PpQDM-Ben & $\mathbf{5 \% ( P V P )}$ & 170 & 80 & 70 & 40 \\
PpQDM-Ben & $\mathbf{5 \% ( P V P )}$ & 170 & 120 & 70 & 50 \\
PpQDM-Ben & 5\%(PVP) & 170 & 140 & 70 & 70 \\
\hline
\end{tabular}


Figure 4.6 (c) indicates that the $r_{33}$ varied simultaneously with the applied voltage and temperature then reached its maximum of $75 \mathrm{pm} / \mathrm{V}$ at the poling field of $70 \mathrm{~V} / \mu \mathrm{m}$. The EO signal increases slowly as temperature is raised. Such a value levelled off under the continuous poling field at $140^{\circ} \mathrm{C}$, resulting in poling efficiency of $1.07(\mathrm{~nm} / \mathrm{V})^{2}$, which is defined as the ratio of $r_{33}$ and $V_{p}$.

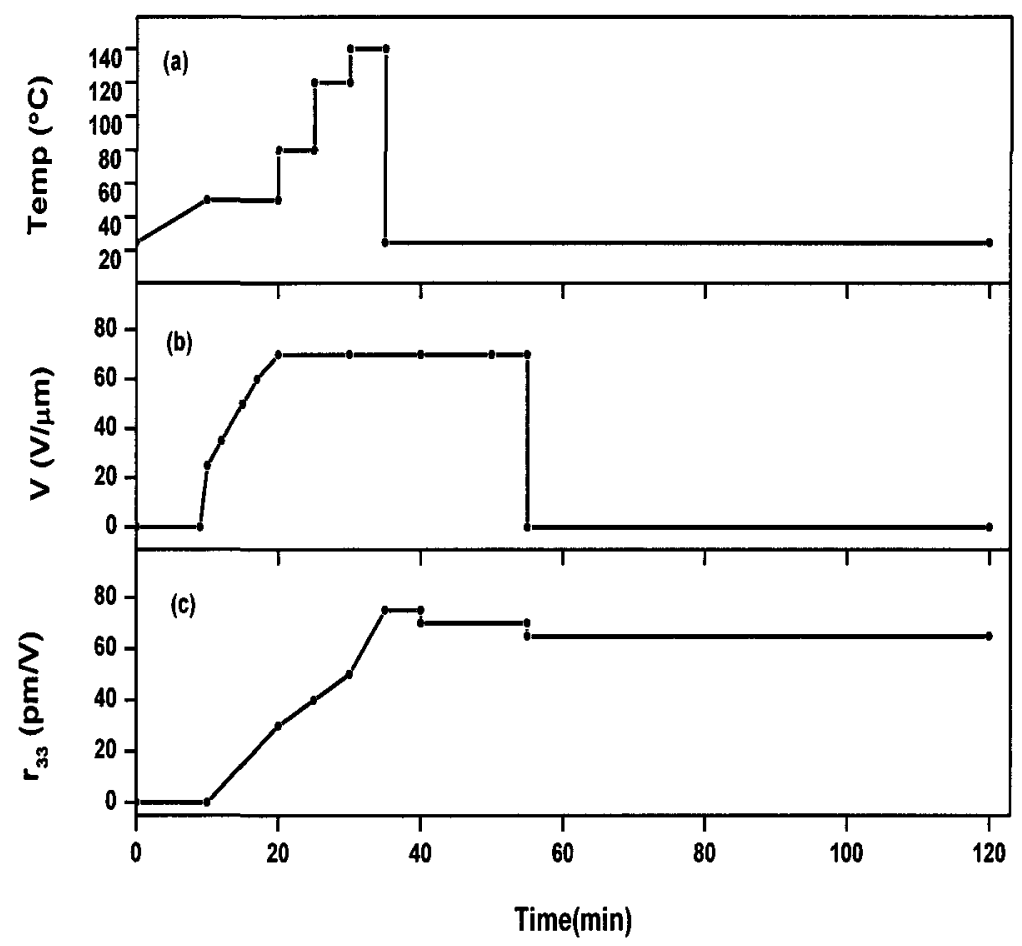

Figure 4.6. In situ poling process plot of 5\% PpQDM-Ben/PVP NLO polymer film. (a) Poling temperature (b) Applied poling field (c) EO coefficient $\left(r_{33}\right)$ value

The sample was then cooled to room temperature with the poling field maintained. During the rapid cooling process, there was a notable relaxation phenomenon of the molecular ordering as the total EO signal decreased by $13 \%$. Eventually, $r_{33}$ of $65-70$ $\mathrm{pm} / \mathrm{V}$ was realized at room temperature for the poled PpQDM-Ben/PVP guest-host film after removing the poling field. Poling efficiency of $1.0(\mathrm{~nm} / \mathrm{V})^{2}$ was estimated for PpQDM-Ben/PVP. 
In situ measurement indicate that materials based on chromophore PpQDMs have larger EO coefficient compared with the FDCNs and PQDM chromophores, especially the chromophore PpQDM-Ben, which contains an additional large benzyl group in the donor part and a suitable shape which leads to a large $r_{33}$ value. This value is the highest value obtained for chromophore PpQDMs in guest-host polymers. All the data indicated that the increased donor strength, and suitable shape of the chromophores could significantly increase their macroscopic EO activities.

\subsubsection{EO Properties of PpQDM-Based Crosslinkable NLO Polyimides}

According to the enhanced molecular hyperpolarizabilities for chromophores PpQDM-Met and PpQDM-Ben compared with PQDM, an improvement of macroscopic EO property for the PpQDM-based crosslinkable NLO polyimides is expected.

The synthesized NLO polyimides, NPI-a to NPI-e offer good processability, and optical quality thin films can be easily prepared by casting onto ITO glass from DMF solution. The polyimides give smooth and physically stable films with thicknesses ranging from 1.4 to $2.2 \mu \mathrm{m}$.

To avoid degradation of the NLO active chromophore during poling, an UV-Vis spectrophotometer was utilized to trace the chromophore in the polymer. Figure 4.7 shows the absorption characteristics of the NLO polyimides NPI-d film after thermal treatment at various temperatures for $30 \mathrm{~min}$. A sharp decrease of the chromophore absorbance was observed when temperatures were higher than $160^{\circ} \mathrm{C}$. This indicates that NLO chromophore is capable of enduring thermal treatment under $160^{\circ} \mathrm{C}$. 

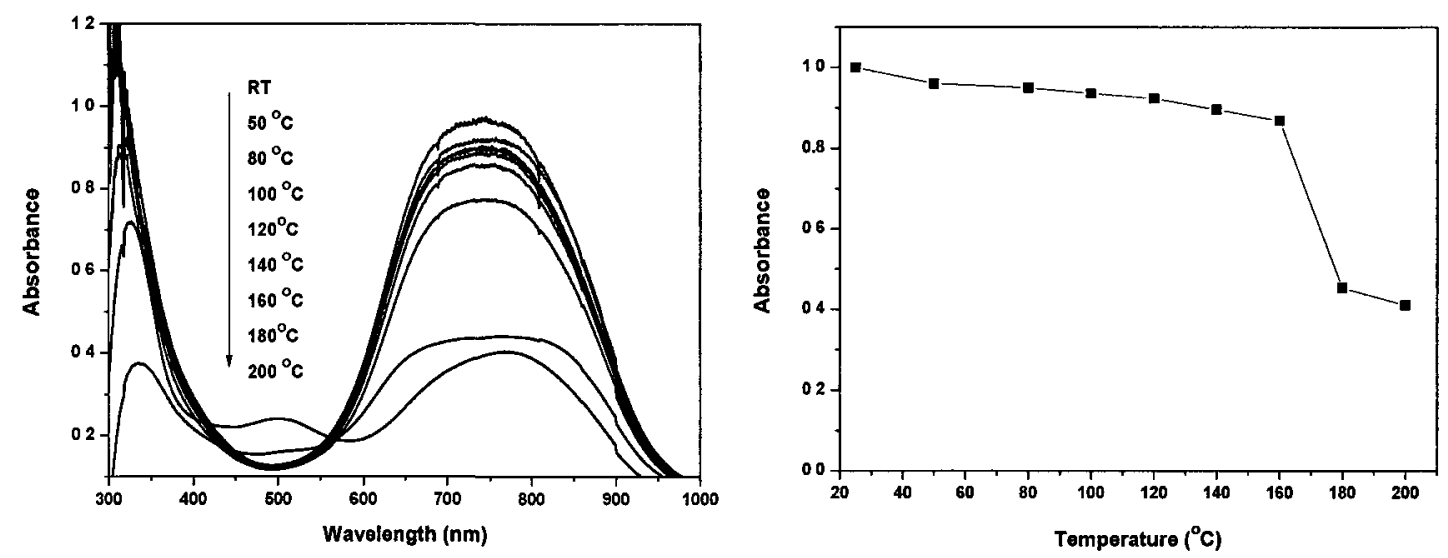

Figure 4.7. UV spectra (left) and thermal stability (right) of NLO polyimide NPI-d thin films.

To evaluate the EO activities of NPI-d and NPI-e, the polymer films were electric poled to induce noncentrosymmetric polar order. To avoid the damages of thermal treatment, electric discharge and the negative effect of crosslinking on the chromophore orientation, electric poling practically involves a process that begins at low temperatures over a long time. For example, NPI-d and NPI-e were initially held at $155^{\circ} \mathrm{C}$ and $160^{\circ} \mathrm{C}$ respectively for 20 min under a poling voltage around $70 \mathrm{~V} / \mu \mathrm{m}$. Electric poling ends with holding at higher temperatures above $170^{\circ} \mathrm{C}$ for around $10 \mathrm{~min}$ while keeping the electric field to complete the crosslinking reaction. The EO coefficients of NPI-d and NPI-e were measured by the simple reflection technique at $1550 \mathrm{~nm}$. NPI-d and NPI-e give 25 and $30 \mathrm{pm} / \mathrm{V}$ of $r_{33}$ value, respectively (Table 4.6).

Table 4.6. Characterization of PpQDM-based crosslinkable NLO polyimide films

\begin{tabular}{cccccccc}
\hline $\begin{array}{c}\text { NLO } \\
\text { polymer }\end{array}$ & $\begin{array}{c}\text { PpQDM } \\
(\mathrm{wt} \%)\end{array}$ & $\begin{array}{c}\mathrm{n} \\
(1.5 \mu \mathrm{m})\end{array}$ & $\begin{array}{c}\text { Film thickness } \\
(\mu \mathrm{m})\end{array}$ & $\begin{array}{c}\mathrm{T}_{\mathrm{g}} \\
\left({ }^{\circ} \mathrm{C}\right)\end{array}$ & $\begin{array}{c}\mathrm{T}_{\mathrm{p}} \\
\left({ }^{\circ} \mathrm{C}\right)\end{array}$ & $\begin{array}{c}\mathrm{V}_{\mathrm{p}} \\
(\mathrm{V} / \mu \mathrm{m})\end{array}$ & $\begin{array}{c}r_{33} \\
(\mathrm{pm} / \mathrm{V})\end{array}$ \\
\hline NPI-d & 7.59 & 1.572 & 1.91 & 163 & 155 & 70 & 25 \\
NPI-e & 13.75 & 1.597 & 2.09 & 180 & 160 & 70 & 30 \\
\hline
\end{tabular}


The higher density of chromophore in NPI-e did not lead to much higher $r_{33}$ value compared to NPI-d with less chromophore, and also the lower EO coefficients of the crosslinkable NLO polyimides compared to PpQDM-Ben doped PVP obtained $\left(r_{33}=70\right.$ $\mathrm{pm} / \mathrm{V}$ ) is mainly due to the chromophore aggregations, as evident by their broader absorptions (See Chapter 3).

Initially, the chromophores might tend to pack with each other to form dimeric aggregates at a relatively low temperature. When the poling temperature was increased, the aggregated chromophores partially dissociated. However, the poling temperature is close to the onset of the crosslinking temperature, partial crosslinking may have taken place, as evident that when the films were heated at $170{ }^{\circ} \mathrm{C}$ for $5 \mathrm{~min}$, they could not be completely dissolved in DMF, which indicate that partial crosslinking occurred in the polymers. This makes it harder for the dissociation of the aggregated chromophores due to the limited mobility of chromophores especially in the high chromophore loading polymers. Moreover, lower poling temperature was applied due to the limitation of the thermal stability of the chromophores, leading to the lower poling efficiency.

In order to improve the poling efficiency of the PpQDM-based crosslinkable NLO polyimides, a conductive polymer, PEDOT: PSS thin film was investigated as the barrier layer for poling the NLO polymers.

In general, for practical optical guided-wave devices application, the NLO polymer layer usually needs to be sandwiched between two cladding layers. The basic requirements for these claddings are: 1) relatively low optical loss, 2) must have refractive indices that are less than that of the active guiding layer, 3) the resistance of the cladding should be significantly smaller than that of the active layer, to make the 
poling voltage in the active layer is nearly equal to the external applied voltage, 4) should be compatible with the processing of the active EO layer. In addition, these cladding layers also act as buffer layers, delaying the onset of dielectric breakdown in the active layer.

The PEDOT: PSS thin film, with thickness around $500 \mathrm{~nm}$ on top of the ITO glass was selected due to its beneficial properties, such as much higher electrical conductivity and dielectric constant, excellent thin film processibility, ${ }^{19}$ and the ability to smooth and pacify rough surfaces such as ITO, reducing charge localization. ${ }^{20}$ To achieve highly transparent and conductive films, a blended system of PEDOT:PSS (Aldrich corporation) in polyvinylalcohol (PVA) was chosen. Solutions of $9 \mathrm{wt} \%$ PVA in water was prepared under $50{ }^{\circ} \mathrm{C}$ first. Then PEDOT: PSS and PVA solutions were mixed at weight ratios varying from 10:100 to 50:100. Solutions were filtered and spin coated onto ITO glass. The resulting films had uniform thickness of approximately $500 \mathrm{~nm}$ and exhibited good transparency. These samples were dried in a vacuum oven at $45^{\circ} \mathrm{C}$ for $48 \mathrm{~h}$.

Transmission was measured because the transparency of the materials used in EO application is critically important. The PEDOT: PSS/PVA films were found to have low absorption in the visible range of the spectrum and had an increased absorption in the infrared range from 1100 to $1500 \mathrm{~nm}$. Figure 4.8 shows that there is a slight decrease in transmission with increasing PEDOT: PSS doping in the blended samples. However, lightly doped samples, $15 \mathrm{wt} \%$ of PEDOT: PSS/PVA had more than $90 \%$ transmission across the measured spectrum. 


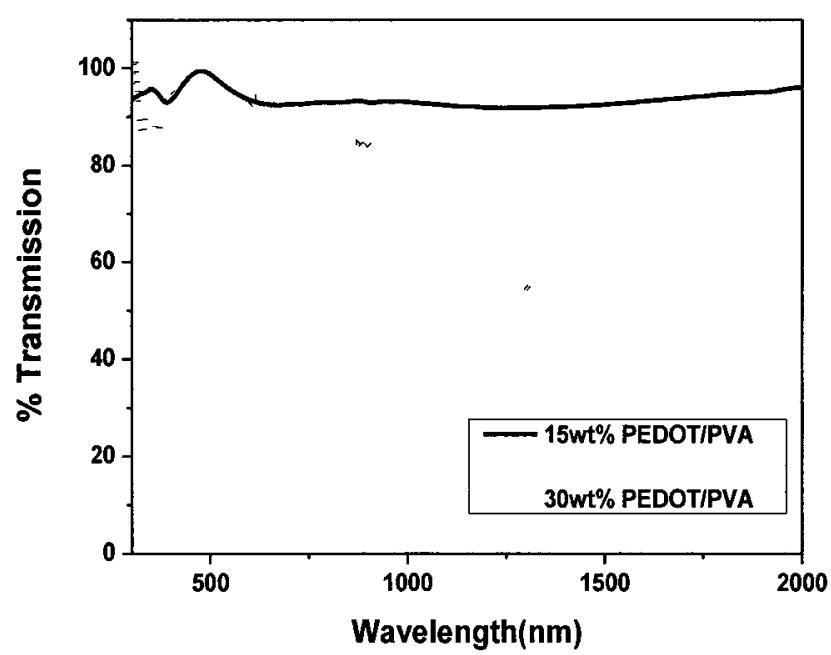

Figure 4.8. UV-Vis-NIR spectra showing optical transparency for PEDOT: PSS/PVA thin films (Thinkness: $500 \mathrm{~nm}$ ).

Therefore, thin films containing $15 \mathrm{wt} \%$ PEDOT: PSS in PVA were prepared on ITO glass for testing as barrier layers for the poling of NLO polymers. Around $2 \mu \mathrm{m}$ of PpQDM-based crosslinkable polyimides were casted on the top of PEDOT: PSS/PVA film as a second layer.
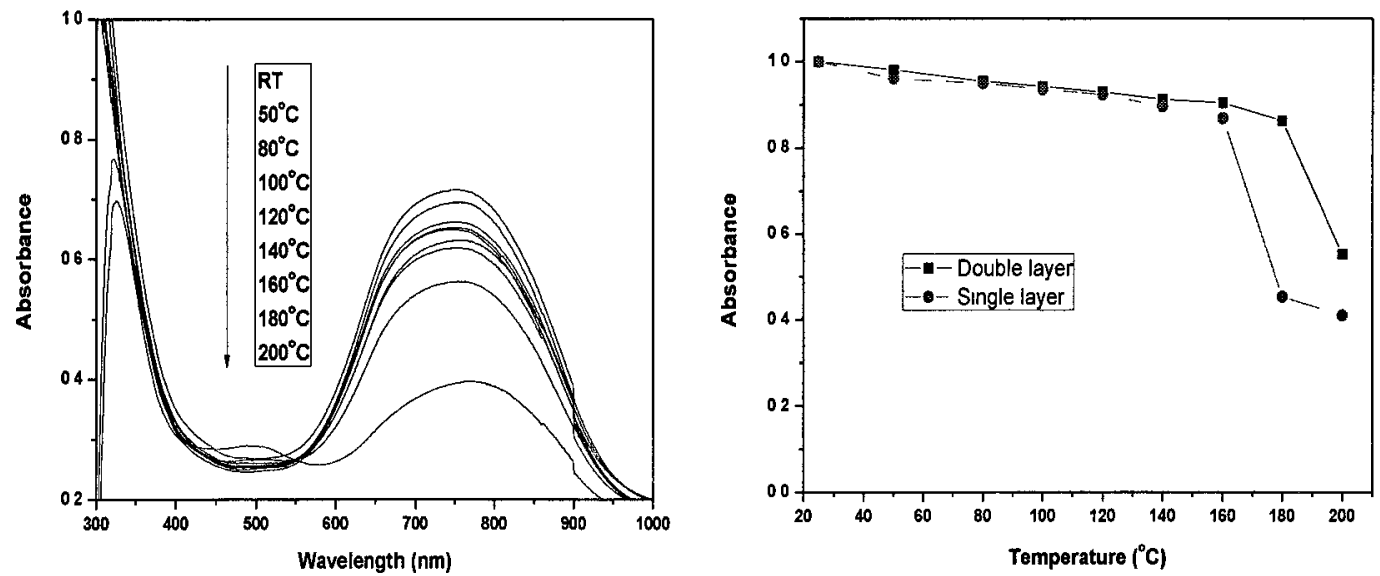

Figure 4.9. UV spectra of NPI-d double layer films (left); thermal stability comparison of the NPI-d in the single and double layer films (right). 
Thermal stability of the chromophores in the double layer films was also tested by UV-Vis absorption. Figure 4.9 (left) shows the absorption characteristics of the NPI-d film after thermal treatment at various temperatures for $30 \mathrm{~min}$. No sharp decrease of the chromophore absorbance was observed till the temperatures reach to $180{ }^{\circ} \mathrm{C}$. This indicates that the NLO chromophores can endure higher temperatures compared with that in the single layer films (Figure 4.9 right).

For comparison, the double layer films were electric poled, the EO coefficients were measured by the simple reflection technique at $1550 \mathrm{~nm}$ as described as the single layer films and the results are summarized in Table 4.7.

Table 4.7. Characterization of PpQDM NLO polymer/PEDOT: PSS/PVA films

\begin{tabular}{cccccc}
\hline $\begin{array}{c}\text { NLO } \\
\text { polymer }\end{array}$ & $\begin{array}{c}\text { PpQDM } \\
(\mathrm{wt} \%)\end{array}$ & $\begin{array}{c}\text { Film thickness } \\
(\mu \mathrm{m})\end{array}$ & $\begin{array}{c}\mathrm{T}_{\mathrm{p}} \\
\left({ }^{\circ} \mathrm{C}\right)\end{array}$ & $\begin{array}{c}\mathrm{V}_{\mathrm{p}} \\
(\mathrm{V} / \mu \mathrm{m})\end{array}$ & $\begin{array}{c}r_{33} \\
(\mathrm{pm} / \mathrm{V})\end{array}$ \\
\hline NPI-d & 7.59 & 1.82 & 170 & 100 & 35 \\
NPI-e & 13.75 & 1.75 & 170 & 100 & 40 \\
\hline
\end{tabular}

Up to $100 \mathrm{~V} / \mu \mathrm{m}$ effective poling fields (defined as the total external applied voltage divided by the thickness of the NLO polymer layer only) were achieved across the NLO polymer layer of the double layer films, while the single layer films could not be poled above $70 \mathrm{~V} / \mu \mathrm{m}$ without encountering dielectric breakdown. Thus, more than $40 \%$ increase in effective poling field could be achieved through the use of the PEDOT: PSS/PVA layers.

The $r_{33}$ value of NPI-d and NPI-e are 35 and $40 \mathrm{pm} / \mathrm{V}$, respectively. With the PEDOT:PSS buffer, the maximum achievable $r_{33}$ values can be enhanced in $\sim 30 \%$ 
higher for both NPI-d and NPI-e NLO polyimides in the double layer films over that observed in the single layer films. The PEDOT: PSS layer which was labeled as conductive in this experiment is much more resistive than electrode such as ITO and gold. This provides a less conductive interface with the active layer than a direct interface with the ITO electrode. Thus the interfacial surface between the NLO polymer and this buffer layer is a relatively low conductivity surface, which diminishes the formation of charge avalanches and reduces the probability of dielectric breakdown.

\subsection{Conclusions}

EO properties of zwitterionic chromophores FDCN and PpQDM, and their corresponding NLO polymers, FDCN-MMA (NA2) and PpQDM-based crosslinkable polyimides have been studied. This work demonstrates that an effective way to reduce the formation of aggregates for FDCN chromophores is by adding a bulky aromatic group side chain perpendicular to the conjugated backbone of the molecule.

The optimum poling conditions for the PpQDM-Ben doped PVP system were studied according to the electrical properties of NLO chromophores and various factors such as polymer matrix, poling temperature and electric poling field. An excellent longterm stability of the induced EO activity of PpQDM-Ben/PVP at $80^{\circ} \mathrm{C}$ was demonstrated.

In situ poling method was established to explore the intrinsic large EO properties of PpQDM series of chromophores. This dynamic EO measurement indicates that the PpQDM-Ben doped PVP NLO materials have the largest EO coefficient $(60-70 \mathrm{pm} / \mathrm{V})$. This result demonstrates that the presence of longer conjugation, stronger acceptor and bulky donor groups in PpQDM significantly enhanced CT interactions, leading to larger 
$\mu \beta$ value than PQDM; also the PVP polymer host has a large enough dielectric constant which favors the dispersion of the polar zwitterionic chromophores.

The poling efficiency of high chromophore concentration crosslinkable NLO polyimides in this study is found to be very sensitive to both temperature and the actual applied field strength. Further modification for PpQDM to become more thermally stable in polymer host is critical to achieve higher poling efficiency.

In this work, a buffer layer (PEDOT: PSS/PVA) was successfully introduced into the double layer device for zwitterionic NLO polymers and improvement of the poling efficiency of NLO polymer systems was demonstrated. More than a $40 \%$ increase in effective poling field was achieved through the use of the buffer layers, also the $r_{33}$ values were enhanced to $\sim 30 \%$ higher for both NPI-d and NPI-e NLO polymers in the double layer films compared with that in the single layer films. The buffer layer provides a relatively low conductivity interface with the active NLO layer in comparison to a direct interface with the ITO electrode, which effectively reduces the probability of dielectric breakdown. By further careful selection of buffer materials, this additional layer will be used to more effectively block the excessive LTC and suppress the onset of dielectric breakdown to enhance the maximum applicable poling field.

\subsection{Experimental Section}

\subsubsection{Materials}

See Chapters 2 and $\mathbf{3}$ for the synthesis of zwitterionic chromophores and NLO polymers, respectively. PMMA, PVP, PVA and PEDOT: PSS 2.8 wt \% dispersed in $\mathrm{H}_{2} \mathrm{O}$ were purchased from Aldrich. PES (commercial trade name: Ultrason $\mathrm{E}, \mathrm{T}_{\mathrm{g}}=225^{\circ} \mathrm{C}$ ) 
was obtained from BASF. The $\mathrm{LiNbO}_{3}$ wafer was purchased from Thorlabs, Inc. (New Jersey, USA) and used to calibrate the Teng-Man setup.

\subsubsection{Thin Film Preparation for EO Study}

Doping NLO chromophore FDCNs and PpQDMs into PES and PVP matrix materials in host-guest system with 1,3 and 5 wt \% chromphore contents; for the cast films, a 2 wt \% DMF solution containing chromophore doped polymers or NLO polymers such as NA2 polymers and crosslinkable NLO polyimides NPI-d to NPI-e was stirred overnight at room temperature in darkness. After the solution was filtered through a $0.2 \mu \mathrm{m}$ syringe filter, it was casted onto ITO glass $\left(2.56 \mathrm{~cm}^{2}\right)$ to produce films of about 1 3 $\mu \mathrm{m}$ thickness and baked at $45{ }^{\circ} \mathrm{C}$ overnight under the protection of nitrogen. The films were further baked in a vacuum oven overnight at $85{ }^{\circ} \mathrm{C}$ to remove the residual solvent. Then a thin gold layer with a thickness of 100-150 nm was sputtered or vacuum deposited on the films as the top electrode for electric poling.

\subsubsection{General Methods}

Refractive indices were measured using a Metricon 2010 prism coupler. An alpha-step 200 surface profiler was used for measuring the thin film thickness. EO coefficients of the poled samples were measured at the laser wavelength of $1550 \mathrm{~nm}$ using the simple reflection technique and a modified Teng-Man reflection method. The Teng-Man setup was calibrated with a commercial $\mathrm{LiNbO}_{3}$ crystals $\left(\mathrm{r}_{33}=31 \mathrm{pm} / \mathrm{V}\right.$ at $1550 \mathrm{~nm}$ ) before use. For the UV-Vis, IR spectrometry, TGA and DSC methods refer to Chapter 2. 


\subsubsection{Poling Process and EO Coefficient Measurement}

\section{Parallel Electrode Poling Station}

The parallel electrode poling setup (Figure 4.1, right) is equipped with a hot plate, whose temperature is sensed by a thermocouple and controlled digitally $\left( \pm 1^{\circ} \mathrm{C}\right)$, and a high voltage supply (0-3 kV) (Bertan Associates, INC. Model 215) (See Appendix C, Figure S4.1). All the components are assembled in a sealed box, through which an inert gas such as nitrogen can flow to remove the air for a high temperature poling. In order to lower the probability of electrical breakdown due to film defects, a half-dumbbell configuration of the gold electrode was made, which has a smaller area than a round disk (See Appendix C, Figure S4.2). During poling and EO measurements, the electrode contact is positioned at the small gold area (diameter $d=2 \mathrm{~mm}$ ), while the incident light is directed at the big gold part $(d=6 \mathrm{~mm})$. A resistor with impedance of $10^{7} \Omega$ is connected in series with the polymer circuit to regulate the poling current.

\section{Teng-Man Setup}

Several techniques are available to evaluate the EO coefficients of poled polymer films. These included attenuated total reflection (ATR), ${ }^{21}$ simple reflection, ${ }^{11 a}$ transmission $^{22}$ techniques and so on. In this research work, the simple reflection technique established by Teng and Man was used (Figure 4.10). The principle of the Teng-Man setup is based on the relative phase shift difference between the $s$ - and $p$ polarized waves of a single laser beam reflected from the sample when a modulating voltage is applied across two parallel plate electrodes. 
The Teng-Man setup was equipped with a $1550 \mathrm{~nm}$ laser (ILX Lightwave LDC 3724B), a Soleil-Babinet compensator (Thorlabs Inc. SBC-IR), a Lock-in amplifier (Standford Research Systems SR830 DSP), a detector (Newport 1830-C), a frequency generator (AVTECH AV-151B-C), an oscilliscope (LeCory 9400A) and other optical components such as a polarizer and an analyzer (See Appendix C, Figure S4.3).

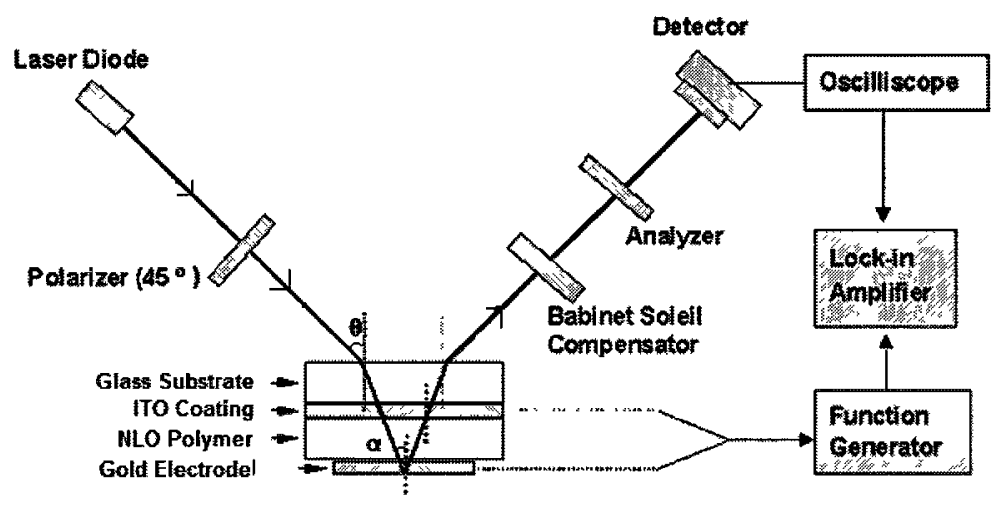

Figure 4.10. Schematic diagram of the simple reflective Teng-Man setup.

\section{EO Coefficient Measurement}

For general measurements of EO coefficient $\left(r_{33}\right)$, the laser beam $(1550 \mathrm{~nm})$ travels through a polarizer, where the incident angle $(\theta)$ was set to be $20^{\circ}$, then passes through the transparent conducting ITO top electrode, and then the EO polymer thin film before being reflected by the bottom gold electrode. The reflected laser beam travels through a compensator and then an analyzer, which is cross-polarized with respect to the polarizer. The modulating voltage $\left(V_{\mathrm{m}}\right)$ is $48.5 \mathrm{~V}$ and the signal frequency is $1 \mathrm{KHz}$ in this experiment. The signals are collected by a detector and then monitored with an oscilloscope and a lock-in amplifier. The intensity of modulated signal $\left(I_{\mathrm{m}}\right)$ and the half intensity of incident light $\left(I_{\mathrm{c}}\right)$ are recorded. 
For in situ EO coefficient measurement, the sample film is mounted under an $\mathrm{N}_{2}$ flow on a temperature-controlled heated stage with good thermal contact to apply the voltage combined of $\mathrm{DC}$ and $\mathrm{AC}$ components. An $\mathrm{AC}$ voltage was added to the $\mathrm{DC}$ voltage for the simultaneous poling and probing of the EO signal $\left(I_{\mathrm{m}} / I_{\mathrm{c}}\right)$. In this measurement, $r_{33}$ signals were collected during the process of heating or cooling in the presence of a poling field (See Appendix C, Figure S4.4). 


\subsection{References}

(1) (a)Sekkat, Z.; Dumont, M. Nonlinear Opt. 1992, 2, 359. (b)Date, M.; Furukawa, T.; Yamaguchi, T. Kojima, A.; Shibata, I. IEEE Trans Dielect. El. In. 1989, 24, 537. (c)Charra, F.; Kajzar, F.; Nunzi, J. M.; Raimond, P.; Idiart, E. Opt. Lett. 1993, 18, 941. (d)Sprave, M.; Blum, R.; Eich, M. Appl. Phys. Lett. 1996, 69, 2962. (e) Burland, D. M.; Miller, R. D.; Walsh, C. A. Chem. Rev. 1994, 94, 31.

(2) Shutoa, Y.; Amano, M.; J. Appl. Phys. 1995, 77, 4632.

(3) (a)Luo, J. D.; Haller, M.; Li, H.; Tang, H.-Z.; Jen, A. K.-Y.; Jakka, K.; Chou, C.H.; Shu, C.-F. Macromolecules2004, 37, 248. (b)Jen, A. K.-Y.; Liu, Y.; Zheng, L.; Liu, S.; Drost, K. J.; Zhang, Y.; Dalton, L. R. Adv. Mater.1999, 11, 452.

(4) (a)Robinson, B. H.; Dalton, R. L. J. Phys. Chem. 2000, 104, 4785. (b)Dalton, L. Adv. Polym. Sci.2002,158,1.

(5) (a)Sprave, M.; Blum, R.; Eric, M. Appl. Phys. Lett. 1996, 69, 2962. (b)Chen, H.; Chen, B.; Huang, D.; Jin, D.; Luo, J. D.; Jen, A. K.-Y.; Dinu, R. Appl. Phys. Lett. 2008, 93, 43507.

(6) Luo, J. D.; Huang, S.; Shi, Z.; Polishak, B. M.; Zhou, X-H.; Jen, A. K-Y. Chem. Mater.2011, 23,544.

(7) Blum, R.; Sprave, M.; Sablotny, J.; Eich, M. J. Opt. Soc. Am. B 1998, 15, 318.

(8) Drummond, J. P.; Clarson, S. J.; Zetts, J. S.; Hopkins, F. K. ; Carraci, S. J. Appl. Phys. Lett.1999, 74,368.

(9) Huang, S.; T.-D.; Luo, J. D.; Hau, S. K.; Shi, Z. W.; Zhou, X.-H.; Yip, H.-L.; Jen, A. K.-Y. Appl. Phys. Lett. 2010,96,2433.

(10) Beaudin, A. M. R.; Song, N. H.; Bai, Y; Men, L.; Gao, J. P.; Wang, Z. Y.; Szablewski, M.; Cross, G.; Wenseleers, W. Campo, J.; Goovaerts, E. Chem. Mater. 2006,18,1079.

(11) Teng, C. C.; Man, H. T. Appl. Phys. Lett. 1990, 56, 1734. (b)Shuto, Y.; Amano, M. J. Appl. Phys. 1995,77,4632.

(12) Robinson, B. H.; Dalton, R. L. J. Appl. Phys. A 2000, 104, 4785.

(13) Naiheng Song "Development of Zwitterinic Nonlinear Optical Polyimides for Electro-Optic Applications", Ph. D. Thesis, Carleton University 2004. 
(14) Zhang, C.; Dalton, L. R. Chem. Mater. 2001,13,3043.

(15) Liakatas, I.; Cai, C.; Bosch, M.; Jager, M.; Bosshard, Ch.; Gunter, P.; Zhang, C.; Dalton, L. R. Appl. Phys. Lett. 2000,76,1368.

(16) Cara Weir "Synthesis and Stability Studies of Z- -(1-Substituted-4-Pyridinium)Cyano-4-Styryldicyano methanide Nonlinear Optical Chromophores", M.Sc. Thesis, Carleton University 2003.

(17) (a)Tsai, H. C.; Yu, I. C.; Chang, P.H.; Yu, D. C.; Hsiue, G. H. Macromol. Rapid Commun. 2007,28,334. (b)Centore, R.; Riccio, P.; Fusco, S.; Carella, A.;

Quatela, A.; Schutzmann, S. J. Polym. Sci. Part A Polym. Chem.2007,45,2719.

(18) Dalton, L. R.; Sullivan, P. A.; Denise H. Bale, D. H. Chem. Rev. 2010, 110, 25.

(19) Drummond, J. P.; Clarson, S. J. Appl. Phys. Lett. 1999, 74, 368. (b) Grote, J. G.; Zetts, J. S.; Nelson, R. L.; Hopkins, F. K.; Dalton, L. R.; Zhang, C.; Steier, W. H. Opt. Eng.2001, 40, 2464.

(20) Carter, S. A.; Angelopoulos, M.; Karg, S.; Brock, P. J.; Scott, J. C. Appl. Phys. Lett. 1997, 70, 2067.

(21) Dentan, V.; Levy, Y.; Dumont, M.; Robin, P.; Chastaing, E. Opt.Commun.1989, 69,379 .

(22) Ma, C. B.; Xu, D.; Ren, Q.; Lv, Z. H.; Yang, H. L.; Meng, F. Q.; Zhang, G. H.; Guo, S. Y.; Sang, L.X.; Wang, Z. G. J. Mater. Sci. Lett.2003, 22, 49. 


\section{Chapter 5 Applications of Zwitterionic Chromophores as Near Infrared Chemosensor}

\subsection{Introduction}

The high sensitivity and abundance of fluorophores makes fluorescence one of the most promising tools for chemosensor development. Numerous chemical and biochemical analytes can be detected by fluorescence methods such as cations, ${ }^{1}$ anions, neutral molecules and gases. ${ }^{2}$ However, the selectivity of fluorescent chemosensors for metal ions remains a significant challenge; also there remains still a need for sensors, which operate and offer minimum perturbation to the microenvironment being probed. Moreover, there is potential for progress in the development of NIR fluorescent sensors for biochemical analytes such as amino acids, coenzymes and nucleotides.

Three series of functional NIR probes have been reported to detect cations over the past decades: Akkaya and Turkyilmaz ${ }^{3 a}$ developed a squaraine-based probe for calcium $\left(\mathrm{Ca}^{2+}\right)$, Akkaya and co-workers ${ }^{3 b}$ also developed a bis(2-pyridiyl)-substituted boratriazaindacene (AzaBODIPY)-based NIR fluorescent probe for mercury $\left(\mathrm{Hg}^{2+}\right)$, and a series of tricarbocyanine derivatives, synthesized by Kazuki Kiyose, ${ }^{3 c}$ were developed into a zinc $\left(\mathrm{Zn}^{2+}\right)$ probe that operates in the NIR region. To the best of our knowledge, no NIR probe has been reported to apply to the detection of both $\mathrm{Fe}^{2+}$ and $\mathrm{Cu}^{2+}$.

PpQDMs are a new series of zwitterionic chromophores, in this thesis their basic photophysical properties were investigated in organic solvents of different polarity (Chapter 2). It is known that these kinds of zwitterionic chromophores are sensitive to protons because of the static affinity between protons and the negative carbon center of 
the chromophore. Therefore, it is predicted that these chromophores might also be sensitive to metal ions.

This chapter will focus on the influence of metal ions on the photophysical properties of PpQDM-Met and application of this chromophore/copper complex as a NIR fluorescent molecular probe for the selective detection of the amino acid-Cysteine.

Part of the work presented in this chapter was published: Hao, W.; McBride, A.; McBride, S.; Wang, Z. Y. "Colorimetric and near-infrared fluorescence turn-on molecular probe for selective detection of cysteine in human plasma", J. Mater. Chem. 2011, 21,1040 .

\subsection{Influence of Metal ions on the Optical Properties of PpQDM-Met}

The investigation of the photophysical properties of PpQDM-Met (M1) as a ligand in the presence of different metal ions has been of particular interest. Its capability to sense the presence of metal ions has been evaluated by monitoring spectrophotometrically the changes in both the absorbance and fluoresence intensity in DMF solution, with regard to potential applications as a NIR metal sensor. DMF was chosen as the solvent for all the measurements since it guarantees a good solubility of the metal salts, ligand used and the respective complexes. The behaviour of $\mathbf{M 1}$ has been tested in the presence of different metal ions including $\mathrm{Li}^{+}, \mathrm{Na}^{+}, \mathrm{K}^{+}, \mathrm{Ag}^{+}, \mathrm{Mg}^{2+}, \mathrm{Ba}^{2+}$, $\mathrm{Mn}^{2+}, \mathrm{Fe}^{2+}, \mathrm{Co}^{2+}, \mathrm{Ni}^{2+}, \mathrm{Cu}^{2+}, \mathrm{Zn}^{2+}, \mathrm{Pb}^{2+}, \mathrm{Hg}^{2+}, \mathrm{Fe}^{3+}, \mathrm{Eu}^{3+}$ and $\mathrm{Er}^{3+}$.

The fluorescence of M1 $\left(2 \times 10^{-5} \mathrm{M}\right)$ is distinguished sensitive to the presence of $\mathrm{Fe}^{2+}$ and $\mathrm{Cu}^{2+}$. For example, a strong decrease of the fluorescence is observed when $2 \times$ $10^{-5} \mathrm{M}$ of $\mathrm{Fe}^{2+}$ was added. Raising the concentration of the $\mathrm{Fe}^{2+}$ up to $20 \times 10^{-5} \mathrm{M}$ induces further decrease in the fluorescence up to $85 \%$ (Figure 5.1). 


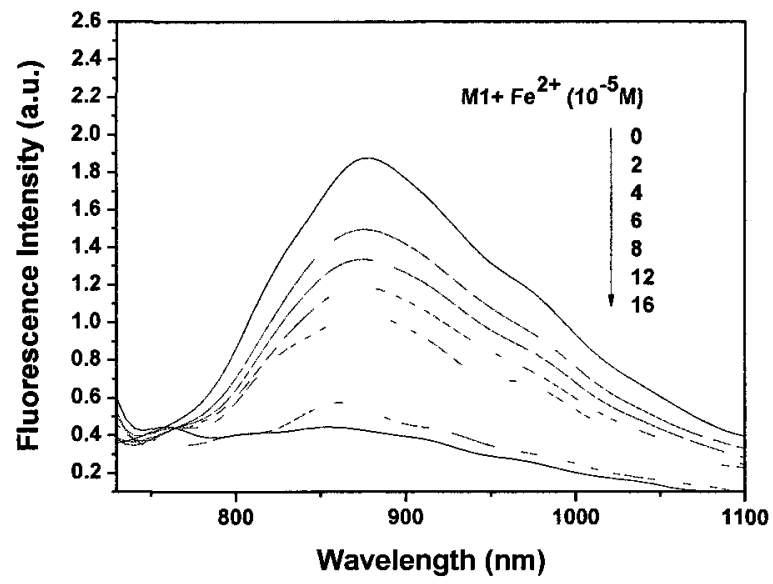

Figure 5.1. Fluorescence spectra of $\mathbf{M 1}\left(2 \times 10^{-5} \mathrm{M}\right)$ in DMF at various concentrations of $\mathrm{Fe}^{2+}$. The concentrations of $\mathrm{Fe}^{2+}$ are increasing from 0 to $1.6 \times 10^{-4} \mathrm{M}$.

The UV-Vis spectrum of M1 in DMF is characterized by a very intense band centered at $680 \mathrm{~nm}$ which is responsible for the blue color of the solution. The absorption at $680 \mathrm{~nm}$ decreased sharply with the gradual addition of $\mathrm{Fe}^{2+}$ to the solution of M1. A new absorption band centered at $480 \mathrm{~nm}$ increased prominently with an isosbestic point at $560 \mathrm{~nm}$, characteristic of the M1-iron (II) complexation. Such a large blue-shift (200 $\mathrm{nm}$ ) makes the color of the solution change from blue to brown-yellow (Figure 5.2).

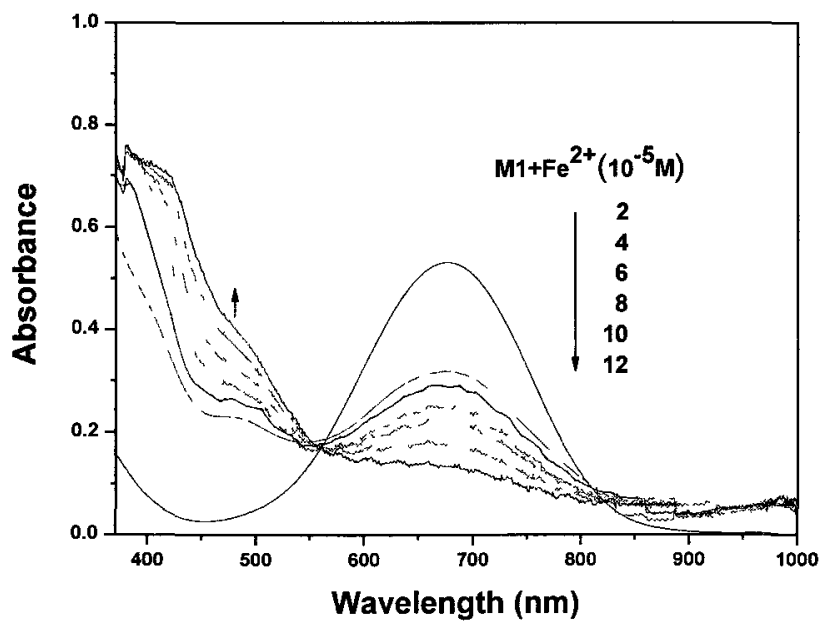

Figure 5.2. Changes in the UV-Vis spectra of $\mathbf{M 1}\left(2 \times 10^{-5} \mathrm{M}\right.$ in DMF $)$ upon titration by $\mathrm{FeCl}_{2}$ from $2 \times 10^{-5}$ to $12 \times 10^{-5} \mathrm{M}$. 
The fluorescence spectra show that the nature of the metal ions determines the behavior of M1 in the complex formation process. The dependence of the metal ions on the fluorescence quenching is presented in Figure 5.3.

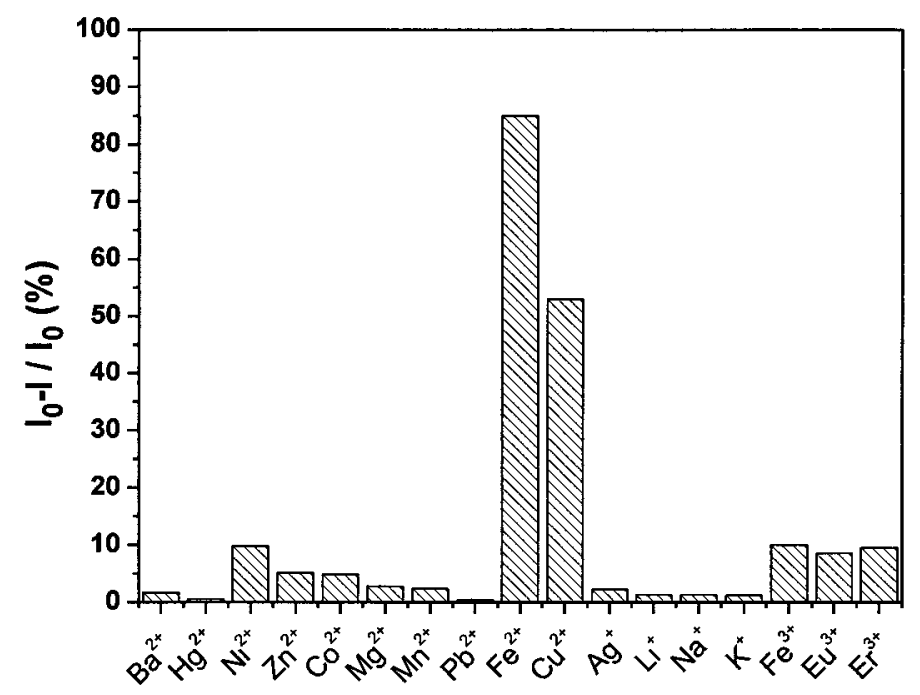

Figure 5.3. Fluorescence responses of $\mathbf{M 1}\left(2 \times 10^{-5} \mathrm{M}\right)$ in the presence of different metal ions $\left(2 \times 10^{-4} \mathrm{M}\right)$ in DMF solution. Excitation wavelength: $670 \mathrm{~nm}$.

The fluorescence quenching factor $\left(\mathrm{I}_{0}-\mathrm{I} / \mathrm{I}_{0}\right)$ was determined from the ratio of maximum fluorescence intensity of M1 before metal ion addition minus the fluorescence intensity after addition of metal ion divided by the maximum fluorescence intensity before addition. The highest fluorescence quenching effect was determined in the presence of $\mathrm{Fe}^{2+}$ and $\mathrm{Cu}^{2+}$ ions ( $85 \%$ and $53 \%$ at $2 \times 10^{-4} \mathrm{M}$, respectively).

There was nearly no difference between the fluorescence intensity of M1 in the presence and absence of alkali $\left(\mathrm{Na}^{+}, \mathrm{K}^{+}\right)$and alkaline earth metal ions $\left(\mathrm{Ba}^{2+}, \mathrm{Mg}^{2+}\right)$. In the case of $\mathrm{Ni}^{2+}, \mathrm{Co}^{2+}, \mathrm{Mn}^{2+}, \mathrm{Zn}^{2+}, \mathrm{Hg}^{2+}, \mathrm{Fe}^{3+}, \mathrm{Pb}^{2+}, \mathrm{Ag}^{+}, \mathrm{Li}^{+}, \mathrm{Eu}^{3+}$ and $\mathrm{Er}^{3+}$, the changes in the fluorescence intensity was also negligible. 
A possible explanation for this effect may be that the ICT process from the donor carbon atom on the negative carbon center to the acceptor bipyridium nitrogen was disturbed by $\mathrm{Fe}^{2+}$ and $\mathrm{Cu}^{2+}$ ions, due to the characteristics of these ions (ionic diameter, charge density, coordination number, hardness of metal ions, etc.). This would weaken the electron density of the negative carbon, causing a fluorescence quenching. The result showed clearly that only $\mathrm{Fe}^{2+}$ and $\mathrm{Cu}^{2+}$ ions were effectively detected.

It is known that most of the identified iron chelators exhibit demonstrably higher affinity for $\mathrm{Fe}^{3+}$ than $\mathrm{Fe}^{2+}$ in physiological conditions, ${ }^{4}$ but selectivity for a particular metal valency cannot always be achievable by metal sensing probes. A commercially available fluorophore, Calcein, with an EDTA-like chelating moiety for metal ion binding, has been applied as turn-off sensor for $\mathrm{Fe}^{2+}$ ion; ${ }^{5}$ a $46 \%$ decrease in Calcein fluorescence was observed upon addition of excess $\mathrm{Fe}^{2+}$, whereas $\mathrm{Fe}^{3+}$ produces a $6 \%$ emission turn-off. Compared with Calcein, large difference between the fluorescence decrease around $90 \%$ for $\mathrm{Fe}^{2+}$ and only $7 \%$ for $\mathrm{Fe}^{3+}$ in $\mathrm{M1}$, making $\mathrm{M1}$ a potentially useful NIR turn-off sensor for $\mathrm{Fe}^{2+}$ ion detection in biosystems. However, $\mathrm{Fe}$ (II) compounds are not stable in ambient conditions as they tend to be oxidized to Fe (III) compounds in air, preventing differentiation between these two oxidation states.

Therefore, the following research turned to focus on the formation of M1 copper complex, then investigated whether the complex could offer some advantages in terms of affinity and selectivity, next attempted to employ the complex as metal-containing receptor for the detection of natural amino acids in the NIR region. 


\subsection{Binding Study of $\mathrm{M1}$ with $\mathrm{Cu}^{2+}$}

Copper is third most abundant (after $\mathrm{Fe}^{3+}$ and $\mathrm{Zn}^{2+}$ ) among the essential heavy metals in the human body and plays an important role in various physiologic processes. ${ }^{6}$ In addition, copper is one of the major components of heavy metal pollution in the environment. It has been found in the range of $7.86 \times 10^{-7} \mathrm{M}$ in non-contaminated environmental samples and up to $62 \mathrm{mM}$ in polluted samples. ${ }^{7}$ The design and synthesis of chemosensors in NIR region for copper ions has become a very active area of research for more sensitive and selective chemosensors for in vitro and in vivo detection. ${ }^{8}$

The binding behaviour of $\mathbf{M 1}$ with $\mathrm{Cu}^{2+}$ in a solution of DMF and methanol $(99: 1$ v/v) was studied using spectroscopic methods. The UV-Vis absorption spectra of a set of solutions containing a constant concentration of M1 $\left(2 \times 10^{-5} \mathrm{M}\right)$ and different amounts of the copper ion were recorded at $25.0 \pm 0.1{ }^{\circ} \mathrm{C}$ (Figure 5.4). Upon gradual addition of $\mathrm{Cu}^{2+}$, the CT band of $\mathrm{M1}$ at $680 \mathrm{~nm}$ decreased dramatically which corresponds to a more intense cation complexation by the $-\mathrm{C}(\mathrm{CN})_{2}$ moiety similar to the effect of protonation. A new absorption band centered at $435 \mathrm{~nm}$ (indicative of the $\mathrm{d}^{-\mathrm{d}^{*}}$ transition of $\mathrm{Cu}^{2+}$ center ${ }^{9}$ increased noticeably with a tight isosbestic point at $500 \mathrm{~nm}$, characteristic of the formation of the M1-Cu complex in DMF solution. A very intense band below $350 \mathrm{~nm}$ can be assigned to the metal ligand charge transfer (MLCT). A large spectral change (245 $\mathrm{nm})$ between M1 and M1-Cu complex or a sharp color change from blue to yellow is ideal for colorimetric detection. 


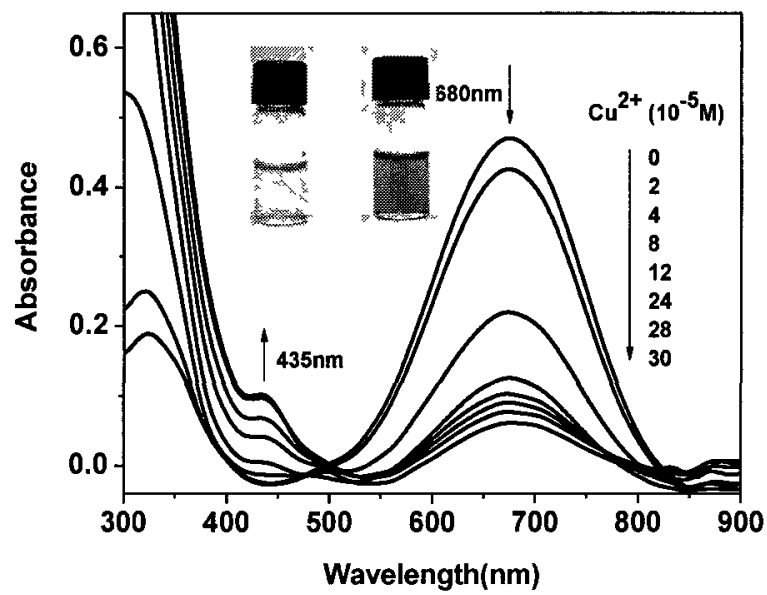

Figure 5.4. Changes in the absorption spectra of M1 in DMF $\left(2 \times 10^{-5} \mathrm{M}\right)$ upon addition of $\mathrm{CuCl}_{2}\left(0\right.$ to $\left.30 \times 10^{-5} \mathrm{M}\right)$.

The fluorescence of $\mathbf{M} 1$ is also very sensitive to the presence of $\mathrm{Cu}^{2+}$. The fluorescence of M1 $\left(2 \times 10^{-5} \mathrm{M}\right)$ was observed to decrease at a low concentration of $\mathrm{Cu}^{2+}$ $\left(4 \times 10^{-5} \mathrm{M}\right)$ and leveled off when 12 equiv. of $\mathrm{Cu}^{2+}$ were added (Figure 5.5 left). A hypsochromic shift in the maximum emission wavelength from $875 \mathrm{~nm}$ to $850 \mathrm{~nm}\left(\Delta \lambda_{\mathrm{F}}=\right.$ $25 \mathrm{~nm}$ ) was observed upon addition of $\mathrm{Cu}^{2+}$, along with significant fluorescence quenching.
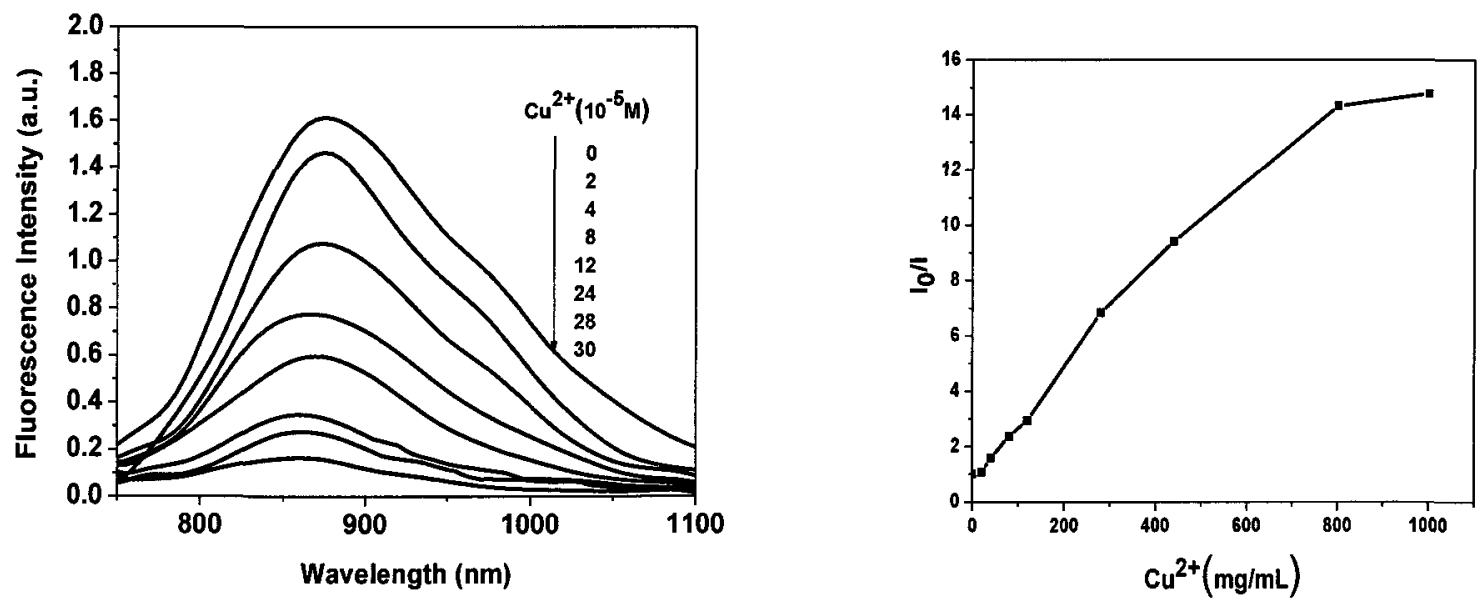

Figure 5.5. Changes in the fluorescence spectra of M1 in DMF $\left(2 \times 10^{-5} \mathrm{M}\right)$ upon addition of $\mathrm{CuCl}_{2}\left(0\right.$ to $\left.30 \times 10^{-5} \mathrm{M}\right)$. 
The influence of other metal ions to the sensing of $\mathrm{Cu}^{2+}$ was also investigated. Figure 5.6 presents the fluorescence spectra of M1 taken in the presence of metal ions $\left(\mathrm{Ni}^{2+}, \mathrm{Co}^{2+}, \mathrm{Mn}^{2+}, \mathrm{Zn}^{2+}, \mathrm{Hg}^{2+}, \mathrm{Fe}^{3+}, \mathrm{Pb}^{2+}, \mathrm{Ag}^{+}, \mathrm{Li}^{+}, \mathrm{Eu}^{3+}, \mathrm{Er}^{3+}, \mathrm{Sr}^{2+}\right.$ and $\left.\mathrm{Mg}^{2+}\right)$ each at concentration of $2 \times 10^{-5} \mathrm{M}$. The fluorescence intensity in this case is slightly lower than that of the solution free of metal ions. The addition of $\mathrm{Cu}^{2+}$ at concentration of $20 \times 10^{-5}$ $\mathrm{M}$ to the same solution causes a dramatic decrease in fluorescence intensity. The data prove that other metal ions resulted in nearly no disturbance to the selective sensing of M1 toward $\mathrm{Cu}^{2+}$ in the NIR region.

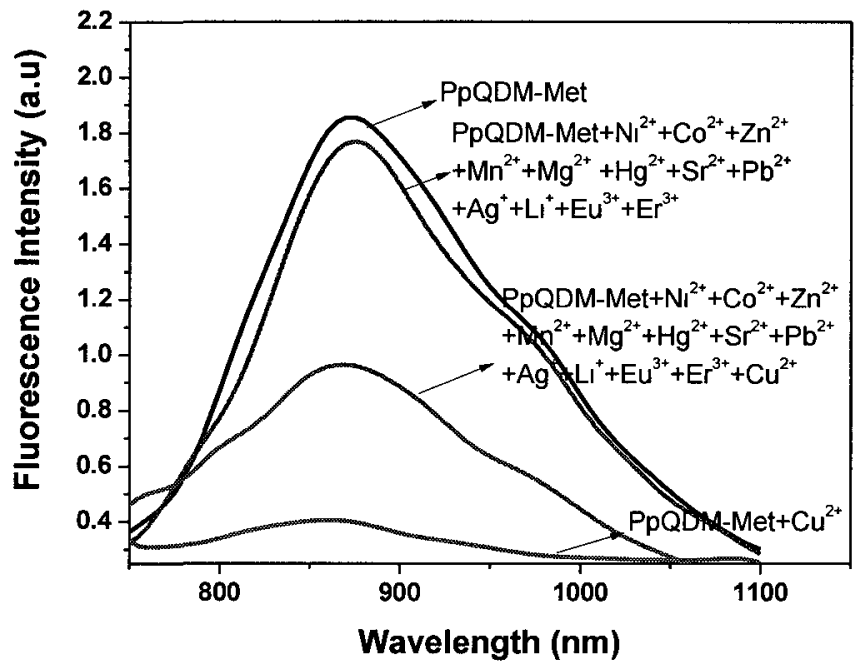

Figure 5.6. Fluorescence spectra of M1 $\left(2 \times 10^{-5} \mathrm{M}\right)$ in DMF at various metal ions.

\subsection{Formation and Characterization of M1-Cu Complex}

In order to confirm the formation of the M1-Cu complex found in the binding study of $\mathbf{M 1}$ with $\mathrm{Cu}^{2+}$, the M1-Cu complex was synthesized and characterized by UV, IR and MS methods.

The synthesis of M1-Cu complex was fairly straightforward and was done simply by mixing a DMF solution of M1 with copper chloride in methanol (Figure 5.7). After 
continuous stirring for 2 hours at room temperature, a yellow complex precipitated out from the solution gradually and was filtered and washed with ethanol and anhydrous ethyl ether. It was isolated as air-stable off-yellow powder in good yield. The complex could be re-dissolved in DMF, affording a yellow color solution with a maximum absorption at $435 \mathrm{~nm}$ (Appendix D, Figure S5.1) but no detectable PL in a range of 700$900 \mathrm{~nm}$.

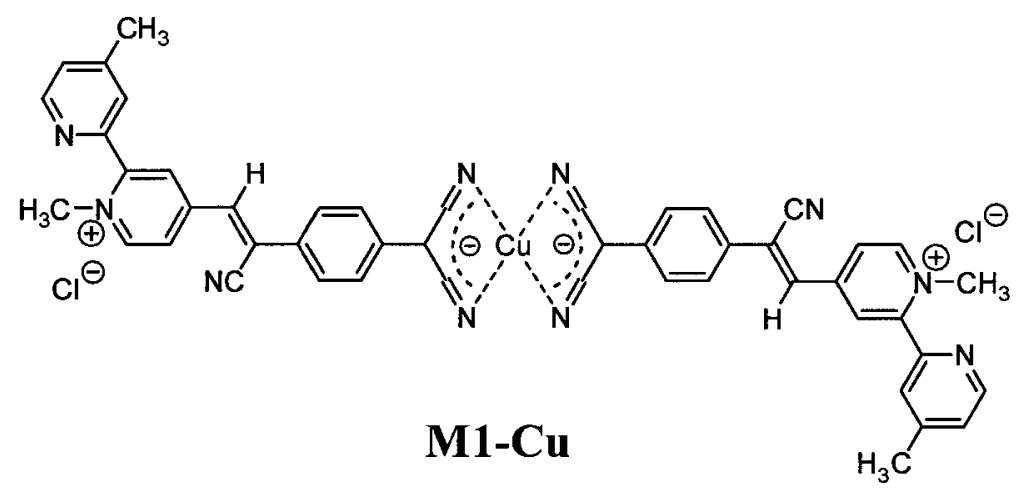

Figure 5.7. Proposed structure of the M1-Cu complex.

The formation of the M1-Cu complex was confirmed by IR spectroscopy. In the IR spectrum of M1-Cu, only a single, relatively weak peak at $2017 \mathrm{~cm}^{-1}$ was observed compared with those two strong absorption peaks at 2173 and $2137 \mathrm{~cm}^{-1}$ in IR spectrum of $\mathrm{M1}^{10}$ (Figure 5.8). The lower intensity of cyano groups in the complex is a consequence of the lower charge intensity on the negative carbon center, indicating charge delocalization through the two cyano groups and possibly onto the empty orbitals of the $\mathrm{Cu}^{2+}$ ion. The significant difference of the $\mathrm{C} \equiv \mathrm{N}$ stretching vibration between the IR spectra of M1 and M1-Cu complex strongly implies that the nitrogen of $-\mathrm{CN}$ groups in M1 are involved in binding with the copper ion. The presence of only one $\mathrm{C} \equiv \mathrm{N}$ stretching vibration at $2017 \mathrm{~cm}^{-1}$ in the isolated M1-Cu complex indicates identical 
bonding of the two cyano groups to the copper in the complex and the relatively lower frequency of the cyano groups further confirms a strong binding with the copper ion. ${ }^{11}$

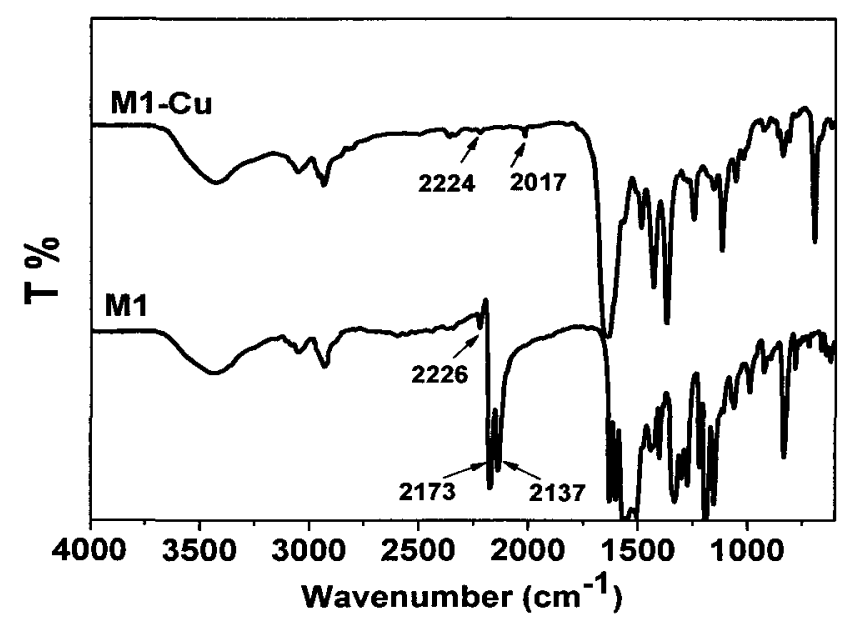

Figure 5.8. Baseline corrected IR spectra $\left(4000-600 \mathrm{~cm}^{-1}\right)$ of $\mathbf{M 1}$ and M1-Cu.

To determine the stoichiometry between $\mathbf{M 1}$ and $\mathrm{Cu}^{2+}$, time-of-flight electrospray ionization mass spectrometry (TOF-ESI-MS) was conducted on the isolated complex. In the ESI mass spectrum, a molecular ion peak at $m / z 882.964$ corresponding to a $2: 1$ complex $\left[2 \mathrm{M1}+\mathrm{CuCl}_{2}\right]^{+}$(calculated value 883.182 ) was clearly observed (Appendix D, Figure S5.2). In addition, fragment peaks at $m / z 375.042$ (calculated value 375.15), 849.119 (calculated value 848.201), and 407.081 (calculated value 406.611) corresponding to $[\mathrm{M1}]^{+},[2 \mathrm{M1}+\mathrm{CuCl}]^{+}$and $[2 \mathrm{M1}+\mathrm{Cu}]^{2+}$ were also present, respectively. Therefore this result confirms the stoichiometry of M1-Cu complex to be $2: 1$.

\subsection{Design of NIR Fluorescence Turn-On Molecular Probe for Cysteine Detection}

Cysteine (Cys, Chart 5.1), a sulfur containing amino acid, facilitates crosslinking of bio-macromolecules through the formation of disulfide bonds in a biological system, which is important in both the structure and function of secondary order proteins. ${ }^{12} \mathrm{~A}$ 
deficiency of Cys is related to many medical conditions including slow growth, hair depigmentation, edema, liver damage, muscle and fat loss, hematopoiesis decrease, leucocyte loss, psoriasis and skin lesions. ${ }^{13}$ Elevated levels of Cys are associated with neurotoxicity, which has been demonstrated in vivo in animals with immature bloodbrain barriers and in cultured neurons in vitro. Cys-induced hypoglycemic brain damage has also been studied as an alternative mechanism to excitotoxicity. ${ }^{14}$ In light of the important roles that Cys plays in a variety of fundamental physiological processes in organisms, there are considerable interests in detecting Cys in biological systems. ${ }^{15}$

The most common determination methods of Cys in human plasma and urine have been developed in conjugation with HPLC, capillary electrophoresis, and gas chromatography, followed by fluorescence or electrochemical detection, and often require time consuming sample separation and preparation. ${ }^{16}$ Because Cys possesses a very low molar extinction coefficient and lacks a suitable chromophore, derivatization with fluorescent reagents are typically used for detection. Most of these fluorescentlabeling reagents contain a functional group, e.g., N-substituted maleimide, reactive halide or aziridine, which reacts with the thiol group in general, and thus are not specific to Cys. ${ }^{17}$ Recent studies have focused on the development of direct fluorescent methods for detection of Cys and other biological thiols, aiming at high sensitivity, remote operation, and suitability as a fluorescent tag for labeling or as an imaging tool for diagnostics. ${ }^{18}$ A fluorescent molecular probe for Cys and homocysteine (Hcy, Chart 5.2) was reported by the Strongin group, ${ }^{19}$ which uses an aldehyde containing xanthene dye to form a thiazolidine by the reaction with $\mathrm{N}$-terminal Cys and Hcy under alkaline conditions resulting in fluorescence quenching. Unlike fluorescence quenching, 
fluorescence turn-on, either increase in fluorescence intensity or a new PL at a different wavelength, is typically more reliable and sensitive method for signaling in detection. ${ }^{20}$

To date, a few research groups have reported fluorescence turn-on sensors for Cys and Hcy. In 2004, Tanaka et al $^{21}$ used a fluorogenic aldehyde as a probe, which reacts with Cys to form a thiazolidine, resulting in an increase in fluorescence at a rather short wavelength of $380 \mathrm{~nm}$. Recently, Huang's group has reported a $588 \mathrm{~nm}$ fluorescence turn-on probe based on the reaction of 8-oxo-8H-acenaphtho[1,2-b]pyrrole-9-carbonitrile with the thiol group in Cys and Hcy. ${ }^{22}$

Clearly, all the reported methods require the chemical reaction of the probing molecule with Cys under specific conditions to form a new product for detection by fluorescence in the visible region. However, the presence of Cys in biological systems and its distribution in cellular processes should ideally be detected quickly, selectively and directly, without any chemical derivatization, by a fluorescence turn-on signaling process in the NIR spectral region.

It is known that among many amino acids, Cys shows the strongest binding ability to $\mathrm{Cu}^{2+}$ over other metal ions, ${ }^{23}$ which suggests its capacity to act as a powerful ligand over others in competitive complexation with $\mathrm{Cu}^{2+}$. Therefore, a fluorescence turn-on probe can be conceptually recognized by the design of a non-florescent complex of $\mathrm{Cu}^{2+}$ and a fluorophore. This probe works simply by release of the fluorophore from its copper complex as a result of ligand exchange with Cys. In addition, the fluorophore should be ideally NIR fluorescent and forms a non-fluorescent copper complex with a relatively weaker binding constant than Cys. 
Zwitterionic chromophore M1 was found to be blue color and NIR fluorescent $(875 \mathrm{~nm})$. Due to the presence of the two cyano groups, binding to $\mathrm{Cu}^{2+}$ produced a nonfluorescent complex and resulted in an immediate color change from blue to brownyellow. Thus, if Cys is able to replace the fluorophore in the copper complex, it should trigger a sharp turn-on signal of NIR fluorescence and a colorimetric signal due to the presence of released fluorophore (Scheme 5.1). Herein we describe the study of M1-Cu complex as a fluorescence turn-on probe for direct, fast, sensitive and selective detection of Cys.

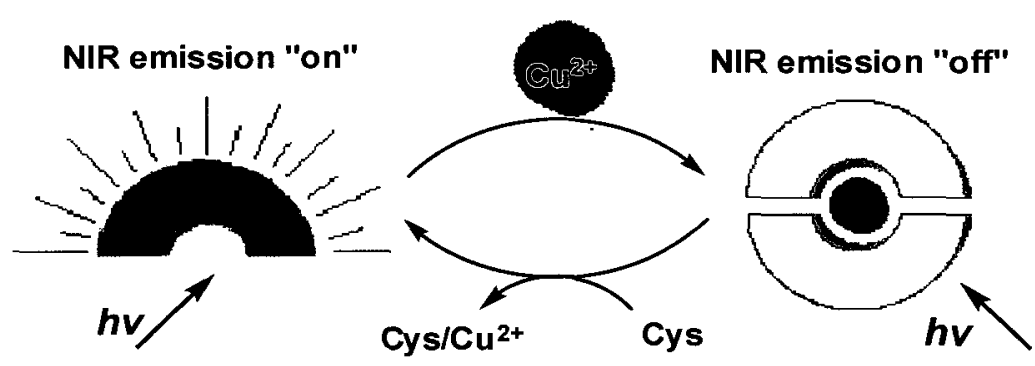

Scheme 5.1. Conceptual design of colorimetric and NIR fluorescence turn-on probe for detection of cysteine, based on the complexation and de-complexation of NIR fluorophore and copper ion.1

Based on the confirmed stoichiometry of a $2: 1\left(\mathbf{M} 1: \mathrm{Cu}^{2+}\right)$ complex and the fluorescence titration data, the binding constant of $\mathbf{M 1}$ with $\mathrm{Cu}^{2+}$ in DMF was calculated to be $4.44 \times 10^{8} \mathrm{M}^{-1}$ (In experimental section). It is important for the M1-Cu complex to have an appropriate binding constant to work as a highly sensitive and selective probe for detection of Cys. Its binding constant should be ideally lower than that of Cys-Cu but higher than those of $\mathrm{Cu}^{2+}$ and many other amino acids and biologically important thiols. 


\subsection{Detection of Cysteine in Solution}

High sensitivity of M1-Cu towards Cys was revealed by fluorescence spectroscopic titration experiments. Figure 5.9 displays the changes in NIR fluorescence of M1 (as a control sample) and M1-Cu in DMF solution at different concentrations of Cys. The fluorescence intensity increases gradually with the increase of the Cys concentration from 0 to $20 \times 10^{-5} \mathrm{M}$ and has a decent signal-to-noise ratio. The release of M1 $\left(2 \times 10^{-5} \mathrm{M}\right)$ due to competitive binding of Cys with $\mathrm{Cu}^{2+}$ is evident by the fluorescence turn-on and the fluorescence recovery percentage (FRP), which is defined as the ratio $\left(\mathrm{I} / \mathrm{I}_{0} \times 100\right)$ of the maximum fluorescence intensity (I) after addition of Cys and the fluorescence intensity $\left(\mathrm{I}_{0}\right)$ of free dye $\mathbf{M 1}$, and correlates well to the concentration of Cys. At the Cys concentration of $4 \times 10^{-5} \mathrm{M}$, the FRP of $\mathbf{M 1}$ was $50 \%$, which reached the maximum value of $89 \%$ when $20 \times 10^{-5} \mathrm{M}$ of Cys was added (Inset of Figure 5.9). A 25$\mathrm{nm}$ bathochromic shift in the maximum emission wavelength from $850 \mathrm{~nm}$ to $875 \mathrm{~nm}$ was also observed upon addition of Cys, which suggested that nearly all M1 was released from M1-Cu.

In order to calculate the detection limit (DL) of M1-Cu for Cys, the intensity values (Figure 5.9) were normalized between the minimum (without Cys) and the maximum intensity $\left(20 \times 10^{-5} \mathrm{M}\right)$. The point at which the line fitting the six values $(20 \times$ $\left.10^{-5} \mathrm{M}-6 \times 10^{-5} \mathrm{M}\right)$ in Figure 5.10 crosses the horizontal ordinate axis is taken as the DL of M1-Cu for Cys, which is approximately $4.07 \times 10^{-6} \mathrm{M}$ (Appendix D ) ${ }^{24}$ 


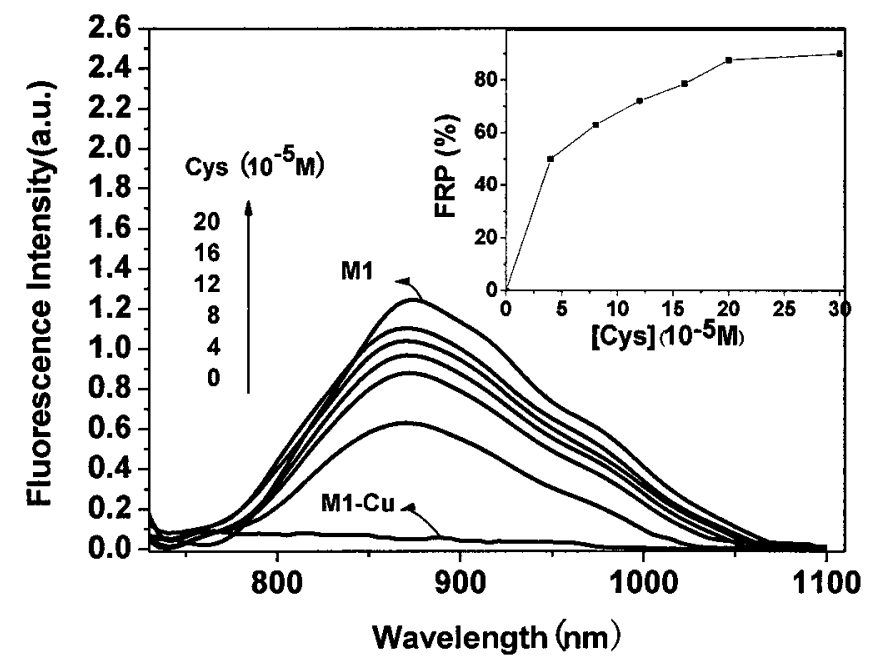

Figure 5.9. Fluorescence spectra of M1-Cu $\left(\mathbf{M 1}=2 \times 10^{-5} \mathrm{M}\right)$ upon addition of Cys $(4 \times$ $\left.10^{-5}-20 \times 10^{-5} \mathrm{M}\right)$ in comparison with the fluorescence spectrum of M1 $\left(2 \times 10^{-5} \mathrm{M}\right)$. Excitation wavelength $=670 \mathrm{~nm}$. Inset: FRP versus the concentration of Cys.

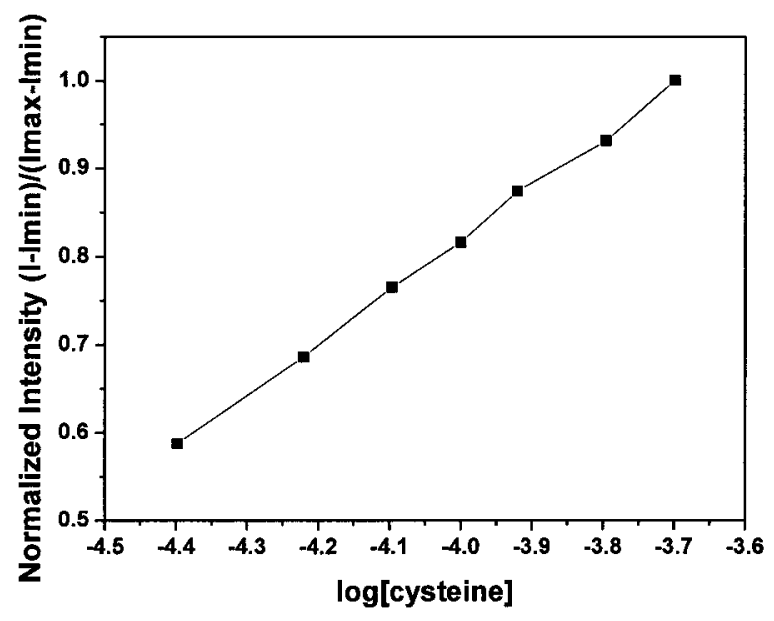

Figure 5.10. Fluorescence intensity of M1-Cu versus Cys concentration.

The M1-Cu response time vs. Cys concentrations is plotted in Figure 5.11. The response time is defined as the time required for the fluorescence signal to reach $90 \%$ of the saturation value after Cys is in contact with M1-Cu. As shown in Figure 5.11, the response time is as short as $10 \mathrm{~s}$ when M1-Cu is exposed to $20 \times 10^{-5} \mathrm{M}$ of Cys, which is, 
to our knowledge, the fastest response of all the reported optical sensors for Cys. $^{20 c, 23 b, 25,26}$

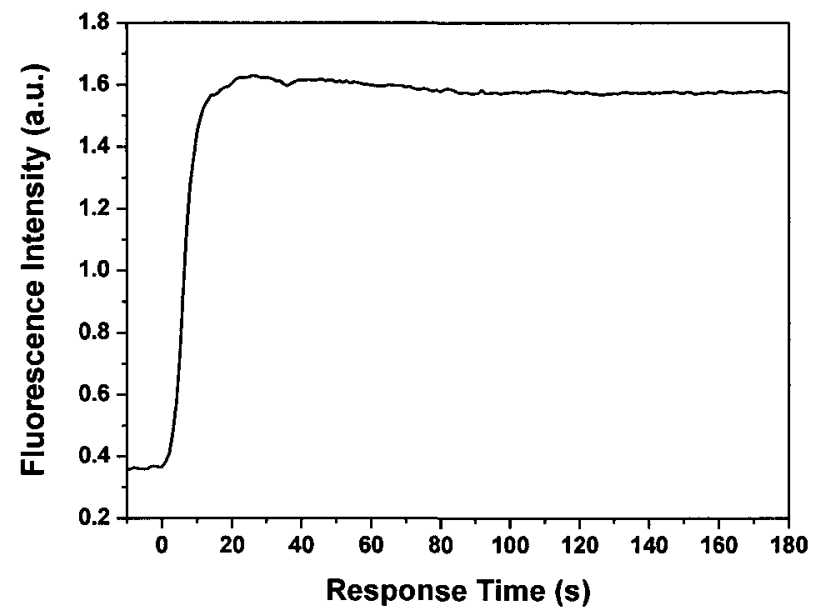

Figure 5.11. Response time measurement for a step change in Cys concentration from 0 to $20 \times 10^{-5} \mathrm{M}$.

The absorption and color changes upon addition of Cys were monitored (Figure 5.12). The absorbance of $\mathbf{M 1 - C u}$ at $435 \mathrm{~nm}$ decreased gradually while the band at 680 $\mathrm{nm}$ of M1 reappeared with increase of Cys. Similar to the fluorescence experiment, the absorbance at $680 \mathrm{~nm}$ reached a maximum when 10 equiv. Cys was introduced. The color changed clearly from brown-yellow to blue and could be observed visually even at the concentration of Cys as low as $4 \times 10^{-5} \mathrm{M}$. 

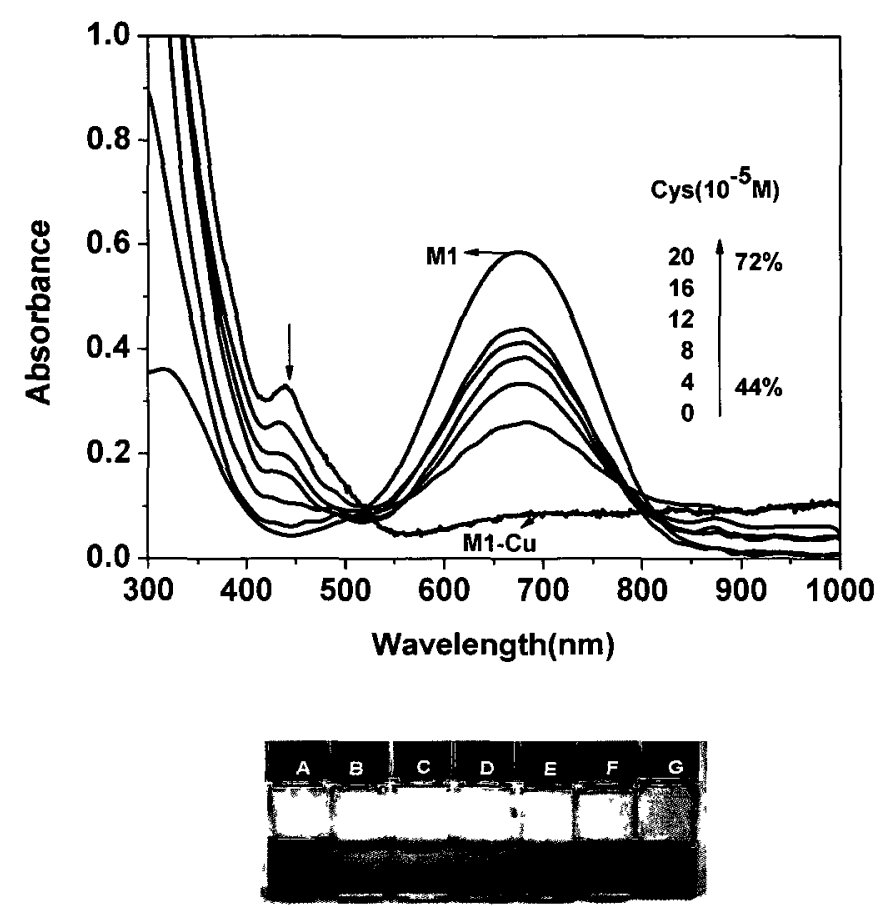

Figure 5.12. Changes in the absorption spectra of M1-Cu $\left(\mathbf{M 1}=2 \times 10^{-5} \mathrm{M}\right)$ upon addition of Cys $\left(4 \times 10^{-5} \mathrm{M}\right.$ to $\left.20 \times 10^{-5} \mathrm{M}\right)$ in comparison with the spectrum of M1 $(2 \times$ $\left.10^{-5} \mathrm{M}\right)$. Photographs of M1 (A: $\left.2 \times 10^{-5} \mathrm{M}\right)$, M1-Cu (B) and M1-Cu containing Cys (C: $4 \times 10^{-5} \mathrm{M}, \mathrm{D}: 8 \times 10^{-5} \mathrm{M}, \mathrm{E}: 12 \times 10^{-5} \mathrm{M}, \mathrm{F}: 16 \times 10^{-5} \mathrm{M}$ and $\left.\mathrm{G}: 20 \times 10^{-5} \mathrm{M}\right)$ in DMF, taken under daylight.

\subsubsection{Selective Detection of Cys in the Presence of other Amino Acids}

Selective detection of Cys in the presence of other amino acids was investigated. Possible interference from naturally occurring $\alpha$-amino acids were first investigated by measuring the absorption and fluorescence spectra of solutions containing these $\alpha$-amino acids $\left(4 \times 10^{-5} \mathrm{M}\right)$ with and without Cys $\left(3 \times 10^{-5} \mathrm{M}\right)$ (Figure 5.13). Among all the $\alpha$ amino acids tested, histidine (His), glycine (Gly), serine (Ser), threonine (Thr), methionine (Met), proline (Pro), alanine (Ala), phenylalanine (Phe), arganine (Arg), lysine (Lys), aspargine (Asp), glutamine (Gln), leuine (Leu), isoleucine (Ile), tyrosine 
(Tyr), glutamate (Glu) and valine (Val), Cys gave the highest response toward the M1-Cu probe.
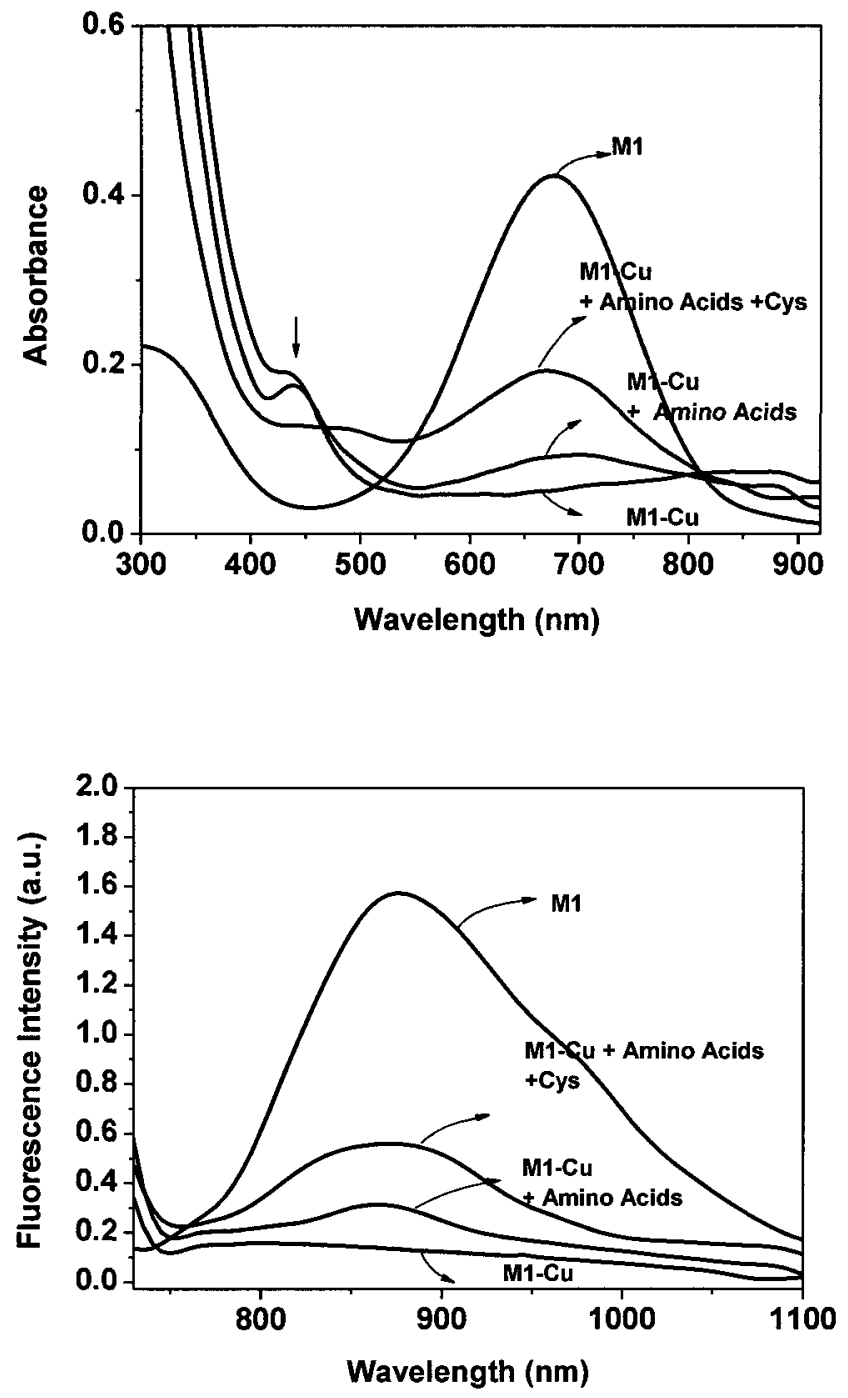

Figure 5.13. Changes in absorption (top) and fluorescence spectra (bottom) of M1-Cu $\left(\mathbf{M 1}=2 \times 10^{-5} \mathrm{M}\right)$ in DMF upon addition of $\alpha$-amino acids $\left(4 \times 10^{-5} \mathrm{M}\right)$ including His, Gly, Ser, Thr, Met, Pro, Ala, Phe, Arg, Lys, Asp, Gln, Leu, Ile, Tyr, Glu and Val, with Cys $\left(3 \times 10^{-5} \mathrm{M}\right)$ or without Cys, in comparison with $\mathbf{M 1}\left(2 \times 10^{-5} \mathrm{M}\right)$.

As shown by the NIR fluorescence changes (Figure 5.14 and Appendix D, Figure S5.3.1 to Figure S5.3.3), the FRP of M1 was the highest at $65 \%$ for Cys at the 
concentration as low as $4 \times 10^{-5} \mathrm{M}, 30 \%$ and $15 \%$ for His and Met, respectively, and below $10 \%$ for the rest $\alpha$-amino acids. The observed order of selectivity towards M1-Cu, Cys $>$ His $>$ Met $>$ other amino acids, is consistent with a trend of ability of the different ligands in biomaterials $(-\mathrm{S}>-\mathrm{N}>-\mathrm{O})$ that can bind to $\mathrm{Cu}^{2+} \cdot{ }^{23}$ The high selectivity is likely due to co-operative effect of the thiol-amino-carboxylic acid moiety in Cys as a ligand to $\mathrm{Cu}^{2+} \cdot{ }^{26}$

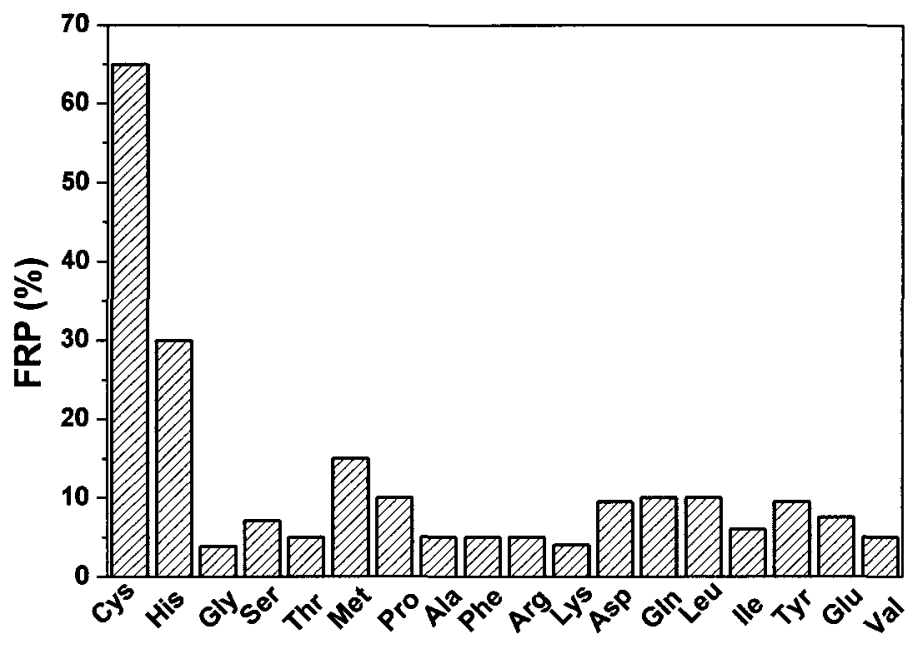

Figure 5.14. Fluorescence recovery percentage (FRP) of M1-Cu $\left(\mathbf{M 1}=2 \times 10^{-5} \mathrm{M}\right)$ towards various natural $\alpha$-amino acids $\left(4 \times 10^{-5} \mathrm{M}\right)($ Excitation at $670 \mathrm{~nm}$, emission at $875 \mathrm{~nm})$.

A relatively stronger binding ability of Cys towards $\mathrm{Cu}^{2+}$ over $\mathrm{M} 1$ in the probe of M1-Cu clearly contributes to high sensitivity in detection of Cys and is also responsible for good selectivity over other amino acids. According to the linear Benesi-Hildebrand expression, $^{27}$ the measured fluorescence intensity $\left[I_{0} /\left(I-I_{0}\right)\right]$ at $875 \mathrm{~nm}$ varied as a function of $1 /$ [amino acid $]^{2}$ in a linear relationship, further confirming $2: 1$ stoichiometry between the amino acids and $\mathrm{Cu}^{2+}$ (Appendix D, Figure S5.4). The binding constants for 
amino acids $/ \mathrm{Cu}^{2+}$ interactions were then calculated and found to be in accordance with the observed selectivity trend (Table 5.1). The binding constant of $\mathrm{Cys} / \mathrm{Cu}^{2+}\left(5.91 \times 10^{8}\right.$ $\mathrm{M}^{-2}$ ) is larger than that of $\mathrm{M1} / \mathrm{Cu}^{2+}$ and those of other amino acids with $\mathrm{Cu}^{2+}$. Therefore, M1-Cu just has an appropriate affinity relative to $\mathrm{Cu}^{2+}$ in comparison with Cys and other amino acids and is able to selectively discriminate Cys among other analogs.

Table 5.1. Binding constants of Cys and some amino acids with copper ion

\begin{tabular}{cc}
\hline Compound & Binding Constant $\left(\mathrm{M}^{-2}\right)$ \\
\hline M1 & $4.44 \times 10^{8}$ \\
Cys & $5.91 \times 10^{8}$ \\
His & $5.54 \times 10^{7}$ \\
Met & $2.63 \times 10^{7}$ \\
Gly & $8.01 \times 10^{5}$ \\
\hline
\end{tabular}

\subsubsection{Effect of Amines and other Biothiols}

Since significant fluorescence changes occur only when Cys is present among other amino acids, the role of the $-\mathrm{SH}$ and $-\mathrm{NH}_{2}$ in $\mathrm{Cu}^{2+}$ binding was further investigated by studying the fluorescence response of M1-Cu with some amines, (e.g., propylamine) and some sulfur-containing compounds such as L-Cystine, ethanethiol and ethyl sulfide (Chart 5.1) with or without the presence of Cys $\left(4 \times 10^{-5} \mathrm{M}\right)$.

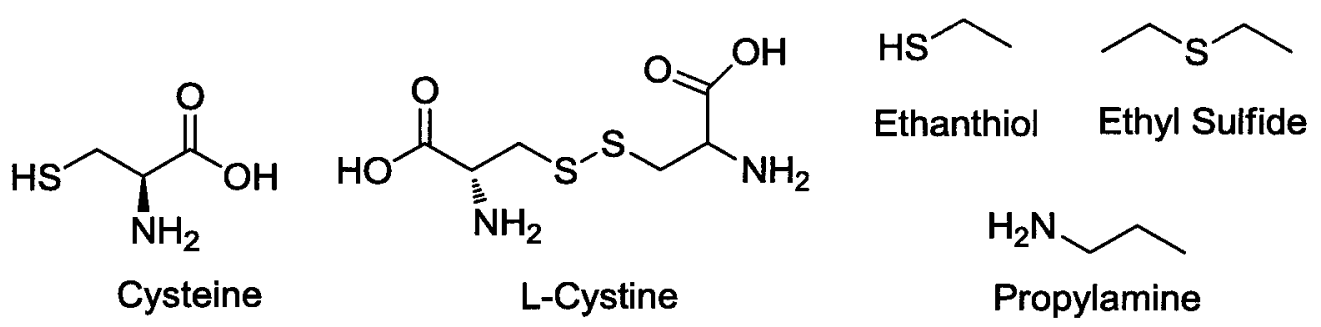

Chart 5.1. Structures of selected competitive chemicals. 
Experimentally, Cys still triggered an insignificant fluorescence increase even when the above potential interfering agents were added in 40 -fold higher concentration than Cys (Figure 5.15). As the precursor of Cys in biosystem, L-cystine does not show any interference in detection. Thus, this experiment clearly confirms that $\mathrm{Cu}^{2+}$ mainly interacts through the co-operative effect of the thiol-amino-carboxylic acid function of Cys, the single functional group such as $-\mathrm{NH}_{2},-\mathrm{SH}$ and $-\mathrm{S}-\mathrm{S}-$ do not bind to $\mathrm{Cu}^{2+}$ effectively, which further explains the high selectivity of the M1-Cu probe towards Cys.

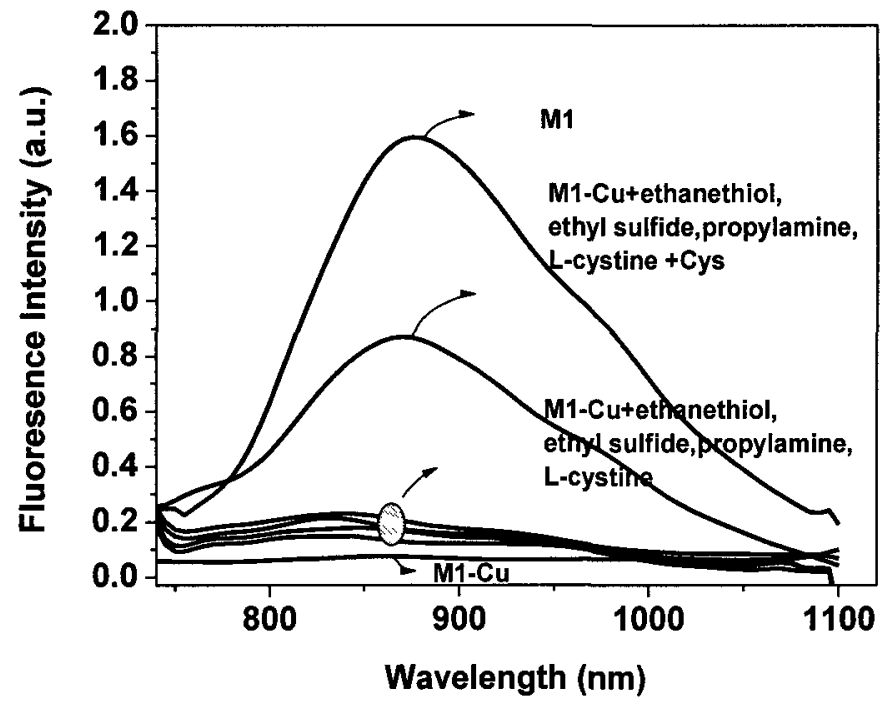

Figure 5.15. Changes in fluorescence spectra of M1-Cu $\left(\mathbf{M 1}=2 \times 10^{-5} \mathrm{M}\right)$ in DMF with various other chemicals including L-cystine, ethanethiol, ethyl sulfide and propylamine $\left(4 \times 10^{-5} \mathrm{M}\right)$ with and without Cys $\left(4 \times 10^{-5} \mathrm{M}\right)$ in comparison with the fluorescence spectrum of $\mathbf{M} 1\left(2 \times 10^{-5} \mathrm{M}\right)$ in DMF.

\subsection{Detection of Cysteine in Human Plasma}

To further demonstrate the potential application of the M1-Cu complex in biological samples, the M1-Cu complex was further studied as a probe to detect Cys in human plasma. It was confirmed that a trace amount of Cys in human plasma can be 
easily detected using M1-Cu by colorimetric, absorption and fluorescence methods. The changes of absorption spectra were observed upon adding human plasma samples spiked with Cys to M1-Cu solution and particularly the intensity of the peak at $680 \mathrm{~nm}$ for M1 was monitored and compared with that of free M1 (Figure 5.16) as a measure for detection limit. With $4 \times 10^{-5} \mathrm{M}$ of Cys present in human plasma, the peak at $680 \mathrm{~nm}$ increased to $40 \%$ intensity of M1 $\left(2 \times 10^{-5} \mathrm{M}\right)$; with $20 \times 10^{-5} \mathrm{M}$ of Cys, the intensity reached to $65 \%$ and at the same time the original blue color of M1 appeared. The overall sensitivity of M1-Cu to Cys in human plasma was only $10 \%$ less than that of in water solution. Similarly, the fluorescence signals were turned on and the emission intensity at $875 \mathrm{~nm}$ increased with an increase of Cys in human plasma (Appendix D, Figure S5.5). The FRP value was found to be $75 \%$ when $20 \times 10^{-5} \mathrm{M}$ of Cys was present. Therefore, the detection limit of M1-Cu for Cys in human plasma is calculated to be $4.37 \times 10^{-6} \mathrm{M}$ (Appendix D, Figure S5.6).

In colorimetric experiments, human plasma samples containing Cys and other $\alpha-$ amino acids were tested using the M1-Cu probe. Visually, only the one containing Cys (4 $\times 10^{-5} \mathrm{M}$ ) in human plasma displayed a distinct blue color that is the same as the standard solution of M1 (Photograph 5.1). The rest samples containing various amino acids remained the original light yellow color like M1-Cu. Therefore, Cys in human plasma as low as $4 \times 10^{-5} \mathrm{M}$ can be readily detected by colorimetric method using the M1-Cu probe. 


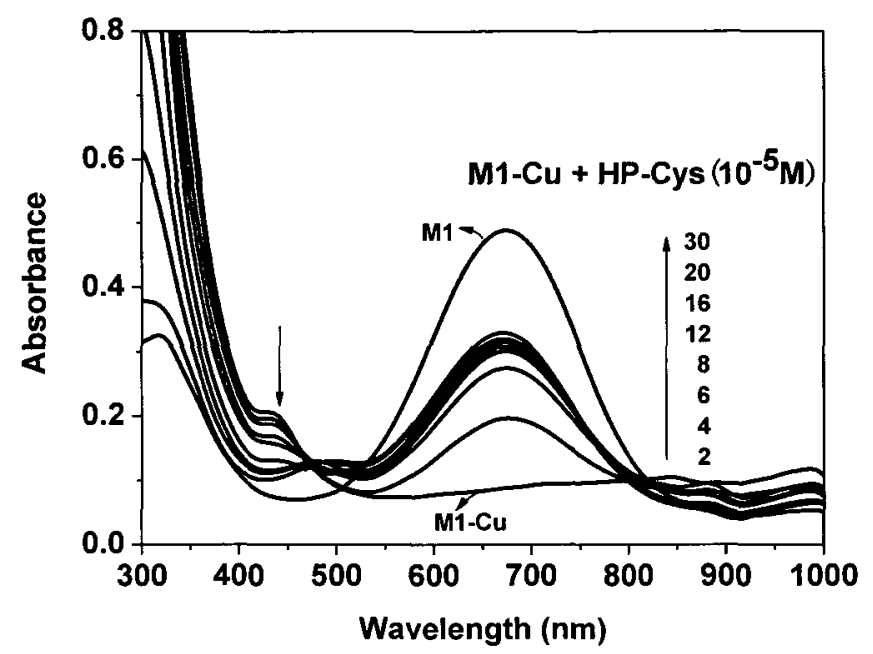

Figure 5.16. Changes in the absorption spectra of M1-Cu $\left(\mathbf{M 1}=2 \times 10^{-5} \mathrm{M}\right)$ upon addition of human plasma with Cys (HP-Cys) in concentrations of $2 \times 10^{-5} \mathrm{M}$ to $30 \times$ $10^{-5} \mathrm{M}$ in comparison with the absorption spectrum of $\mathbf{M 1}\left(2 \times 10^{-5} \mathrm{M}\right)$.

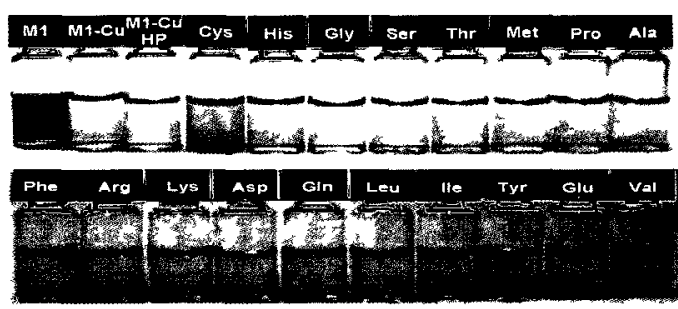

Photograph 5.1. Solutions of M1 $\left(2 \times 10^{-5} \mathrm{M}\right), \mathbf{M 1 - C u}\left(\mathbf{M 1}=2 \times 10^{-5} \mathrm{M}\right), \mathbf{M 1}-\mathrm{Cu}$ in human plasma (M1-Cu HP), and M1-Cu in human plasma samples containing Cys and various amino acids, taken under daylight. The concentration of each amino acid is fixed at $12 \times 10^{-5} \mathrm{M}$.

Selective detection of Cys in human plasma with various $\alpha$-amino acids was experimentally confirmed by titration of M1-Cu with human plasma solutions spiked with various amino acids with or without of Cys over a range of 0 to $30 \times 10^{-5} \mathrm{M}$ (Figure 5.17). The ratio of amino acids to Cys is varied and the concentrations of Cys in human plasma samples are also varied as $0 \mathrm{M}$ (HPAA), $10 \times 10^{-5} \mathrm{M}(\mathrm{HP} 21), 15 \times 10^{-5} \mathrm{M}$ (HP11), $20 \times 10^{-5} \mathrm{M}(\mathrm{HP} 12)$ and $25.8 \times 10^{-5} \mathrm{M}$ (HP13). There was a negligible change in 
both absorption and fluorescence spectra when human plasma with other amino acids (HPAA, $30 \times 10^{-5} \mathrm{M}$ ) without Cys was added to M1-Cu in DMF solution. For the sample of HP21, there was a small change in both absorbance and fluorescence emission. As the concentration of Cys increases, both absorbance and fluorescence were further enhanced. The FRP of M1 with HP13 sample $\left(25.8 \times 10^{-5} \mathrm{M}\right.$ of Cys) was higher than that standard sample of human plasma containing only Cys $\left(20 \times 10^{-5} \mathrm{M}\right)$ (Figure 5.17, Right). Therefore, this probe can be used to selectively detect Cys in relatively more complex native human plasma.
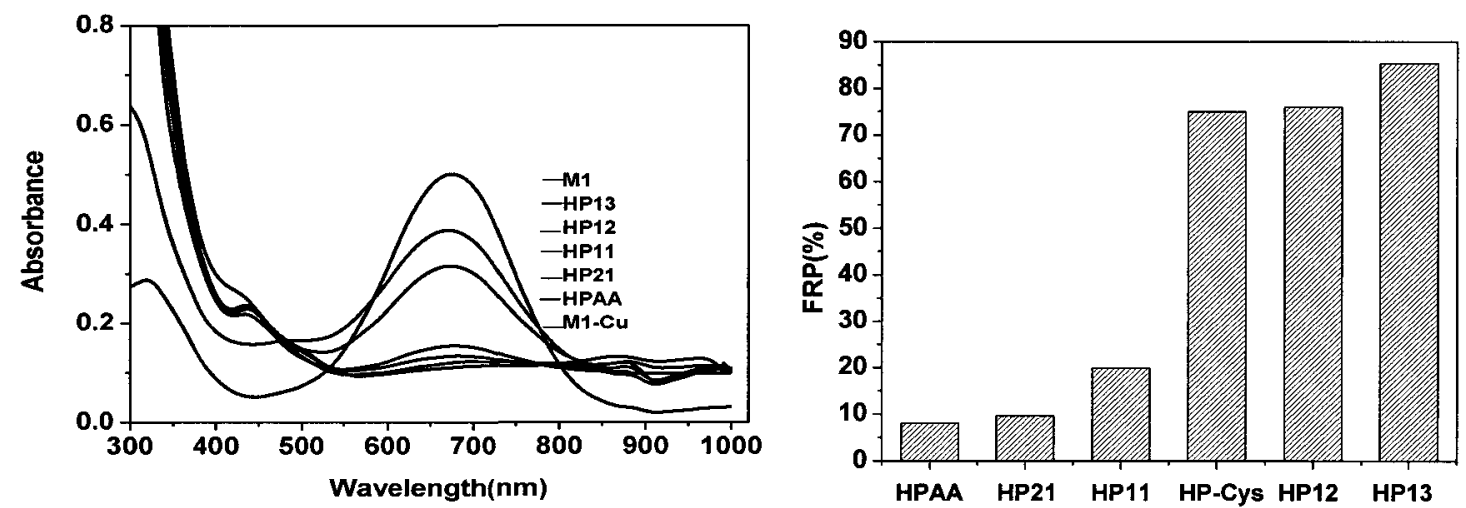

Figure 5.17. Left: UV-Vis absorption spectra of M1 $\left(2 \times 10^{-5} \mathrm{M}\right), \mathbf{M 1}-\mathbf{C u}\left(\mathbf{M 1}=2 \times 10^{-5}\right.$ $\mathrm{M}$ ) and the titration of M1-Cu with deproteinized plasma solutions spiked with various amino acids with or without of Cys. Total amino acids concentration is fixed at $30 \times 10^{-5}$ M. The ratio of amino acids to Cys is varied in plasma samples: HPAA = human plasma with amino acids (His, Gly, Ala, Val, Ile, Pro, Met, Asp, Ser, Phe, Thr, Glu, Lys, Arg, Gln, Leu and Tyr $)\left(30 \times 10^{-5} \mathrm{M}\right)$ without Cys; HP21 = 2:1 (Cys $\left.=10 \times 10^{-5} \mathrm{M}\right), \mathrm{HP} 11=$ $1: 1\left(\mathrm{Cys}=15 \times 10^{-5} \mathrm{M}\right), \mathrm{HP} 12=1: 2\left(\mathrm{Cys}=20 \times 10^{-5} \mathrm{M}\right)$, and HP13 = 1:3 $(\mathrm{Cys}=25.8 \times$ $10^{-5} \mathrm{M}$ ). Right: Fluorescence recovery percentage at $875 \mathrm{~nm}$ (excitation at $670 \mathrm{~nm}$ ) of the M1-Cu probe (M1 $\left.=2 \times 10^{-5} \mathrm{M}\right)$ with the above deproteinized plasma solutions HPAA, HP21, HP11 and HP13. The concentration of Cys in human plasma for the control sample of HP-Cys is $20 \times 10^{-5} \mathrm{M}$. 
Finally, detection of Cys among other biologically important thiol compounds, such as Hcy, L-glutathione (GSH) and N-acetylcysteine (N-Acys) was studied (Chart 5.2). Cys can be obtained as the final product of the transsulfuration pathway through Hcy metabolism. Hcy is a risk factor for disorders including cardiovascular ${ }^{28}$ and Alzheimer's disease. ${ }^{29}$ Unlike Hcy, the relatively more common biological thiols, GSH is widely distributed in animal tissues and plasma, which plays essential roles in the maintenance of the integrity of thiol moieties of protein and other compounds, is typically associated with beneficial antioxidant activity. ${ }^{30}$

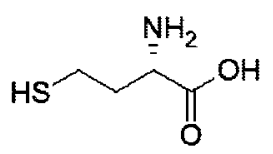

Homocysteine

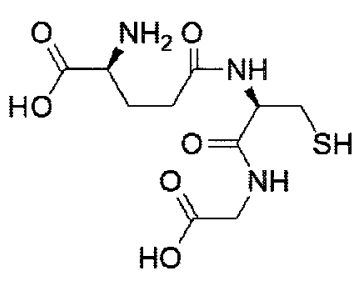

L-Glu tath ıone

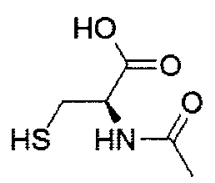

N-Acetylcyste ine

Chart 5.2. Structures of selected bioactive thiols.

Therefore, it is of interest to exam the sensitivity and selectivity of the M1-Cu probe for detection of Cys, Hcy, N-Acys and GSH. Comparative fluorescence studies of Cys and bioactive thiols at a concentration level of $4 \times 10^{-5} \mathrm{M}$ indicated a relative sensitivity for detection with M1-Cu in the following order: $\mathrm{Cys}>\mathrm{GSH}>\mathrm{Hcy}>\mathrm{N}$-Acys (Appendix D, Figure S5.7). Without the active amino group, N-Acys is the most inert towards the probe among the four biothiols. It is known that in healthy human plasma the total Hcy concentration is less than ca. $12-15 \mu \mathrm{M}$, and Cys concentration is typically 2030 times that of $\mathrm{Hcy}^{19}$ and 10 times that of $\mathrm{GSH}^{30}$ Since the M1-Cu probe can selectively monitor Cys in a range of $10^{-4}-10^{-5} \mathrm{M}$, Hcy only becomes detectable by the same probe when its concentration reaches a dangerous level of $1.3 \times 10^{-4} \mathrm{M}^{19 \mathrm{~b}}$ Therefore, 
the M1-Cu probe is potentially useful for sensitive and selective detection of Cys in biological system.

\subsection{Conclusions}

A new zwitterionic NIR fluorophore, M1 has been synthesized and investigated. The influence of metal ions $\left(\mathrm{Cu}^{2+}, \mathrm{Fe}^{2+}, \mathrm{Ni}^{2+}, \mathrm{Co}^{2+}, \mathrm{Mn}^{2+}, \mathrm{Zn}^{2+}, \mathrm{Hg}^{2+}, \mathrm{Fe}^{3+}, \mathrm{Pb}^{2+}, \mathrm{Ag}^{+}\right.$,

$\mathrm{Li}^{+}, \mathrm{Eu}^{3+}, \mathrm{Er}^{3+}, \mathrm{Mg}^{2+}$ and $\mathrm{Sr}^{2+}$ ) on the fluorescence intensity has been studied to test the capacities of M1 as both a NIR fluorescence and a naked eye sensor for these ions in the environment. The data obtained shows that the new M1 is capable of detecting selectively $\mathrm{Cu}^{2+}$ and $\mathrm{Fe}^{2+}$ ions in DMF solution. The detection is based on both colorimetric detection and the quenching of ICT processes in the M1 fluorophore.

A rational strategy for the development of a zwitterionic-metal dependent optical sensing material was demonstrated. The unique ability of $\mathbf{M 1}$ to form a copper complex has led to the design and development of the first colorimetric and NIR fluorescence turnon molecular probe, M1-Cu, for sensitive and selective detection of Cys in human plasma, in the presence of various amino acids and biologically active thiol compounds. The M1-Cu probe offers high-contrast colorimetric and unique NIR fluorescence turn-on methods for Cys detection with a detection range of $10^{-5}-10^{-4} \mathrm{M}$ within a normal physiological level.

\subsection{Experimental Section}

Materials. All organic solvents were of spectroscopy grade and used without further purification. Salts of metals were purchased from Aldrich Chemical Co., The British Drug House LTD., Bioshop Canada Inc. including anhydrous copper (II) chloride, nickel 
(II) chloride hexahydrate, cobalt (II) acetate tetrahydrate, manganese (II) acetate, zinc nitrate, mercuric chloride, iron (II) chloride tetrahydrate, iron (III) chloride, lead (II) acetate trihydrate, barium acetate, magnesium sulfate, silver nitrate, lithium bromide, sodium chloride, potassium chloride, europium chloride and Erbium chloride. Amino acids, L-cystine, propylamine, ethanethiol, ethyl sulfide and other chemicals were purchased from Aldrich Chemical Canada. Pooled human plasma (sodium citrate as an anticoagulant) was purchased from Bioreclamation Inc. (U.S.A.) and was stored at $-20^{\circ} \mathrm{C}$ until analysis. Distilled water was used in all experiments.

Methods. The UV-Vis absorbance spectrophotometric investigation was performed on a Perkin-Elmer Lambda 900 UV-Vis-NIR spectrometer. Fluorescence emission spectra were measured on a PTI fluorescence system. The absorption and fluorescence emission spectra of all the samples were taken in $\mathrm{DMF} / \mathrm{MeOH}$ solution in a quartz cuvette with a path length of $10.0 \mathrm{~mm}$.

Fluorescence and $\mathrm{UV}-\mathrm{Vis}$ absorption titration of $\mathrm{M1}$ with $\mathrm{Cu}^{2+}$. A solution of $\mathrm{M1}$ (2 $\left.\times 10^{-5} \mathrm{M}\right)$ in DMF and a solution of $\mathrm{CuCl}_{2}\left(2 \times 10^{-1} \mathrm{M}\right)$ in $\mathrm{MeOH}$ were prepared and used for all of the following experiments. The titration was carried out by sequentially adding $1 \mu \mathrm{L}$ of aliquots of $\mathrm{Cu}^{2+}$ solution to $10 \mathrm{~mL}$ of the $\mathbf{M} 1$ solution. The solutions of M1 with $\mathrm{Cu}^{2+}$ were stirred for 1 minute and were placed in a quartz cuvette. The absorption and fluorescence spectra were recorded at room temperature each time (excitation wavelength $=670 \mathrm{~nm})$.

Determination of binding constant of $\mathbf{M 1}-\mathrm{Cu}^{2+}$. To determine the binding strengths of the M1-Cu complex, a series of the M1 solutions at a fixed concentration were mixed with the $\mathrm{Cu}^{2+}$ solutions at various concentrations. Absorbance of the resultant mixtures at 
$680 \mathrm{~nm}$ was measured. Fluorescence emission intensity of the resultant mixtures at 875 nm was measured. All measurements were carried out in DMF: Methanol = 99:1 (v/v). Same conditions are used for all of the fluorescence emission intensity measurement. The binding constant of the $\mathrm{M1}$ with $\mathrm{Cu}^{2+}$ was calculated according to the $\mathrm{B}-\mathrm{H}$ equation for spectrofluorometric titration.

Selectivity of M1-Cu to Cys Measurements. The concentration of M1 in M1-Cu is $2 \times$ $10^{-5} \mathrm{M}$. Amino acids were added in portions $(2-35 \mu \mathrm{L})$ to $10 \mathrm{~mL}$ of $\mathbf{M 1 - C u}$ solution. The fluorescence intensity changes were recorded at room temperature each time. Amino acids $\left(2 \times 10^{-1} \mathrm{M}\right.$ in water) used in experiments are Cys, His, Gly, Ala, Val, Ile, Pro, Met, Asp, Ser, Phe, Thr, Glu, Lys, Arg, Gln. Leu $\left(8 \times 10^{-2} \mathrm{M}\right)$ and Tyr $\left(8 \times 10^{-3} \mathrm{M}\right)$ were used. Response Time Measurement. The response characteristic of $\mathbf{M 1 - C u}$ probe was measured in DMF: Methanol = 99:1 (v/v) solution, concentration of M1 in M1-Cu was 2 $\times 10^{-5} \mathrm{M}$. After addition of $20 \times 10^{-5} \mathrm{M}$ of Cys, the fluorescent signal was monitored.

Determination of $\mathrm{Cu}^{2+}$-amino acid binding constant. For the determination of binding strengths of the various $\mathrm{Cu}^{2+}$-amino acid adducts, a series of M1-Cu complex solutions at a fixed concentration were mixed with the amino acid solutions at various concentrations. Absorption of the resultant mixtures at $680 \mathrm{~nm}$ and fluorescence intensity of the resultant mixtures at $875 \mathrm{~nm}$ were measured.

Binding constants of $\mathrm{Cu}^{2+}$ with amino acids using the Benesi-Hilderbrand method. Binding constants, $K_{\mathrm{B}}$, were estimated from the ratio between the $y$-intercept and the slope of the linear line of best fit. The 1:2 donor-acceptor interactions were analyzed according to the B-H equation for spectrofluorometric titration (eq.1). 


$$
\frac{I_{0}}{I-I_{0}}=\left(\frac{c}{d-c}\right)^{2}\left(\frac{1}{K_{B}[\text { substrate }]^{2}}+1\right)
$$

where $I_{0}$ and $I$ are luminescence intensity of $\mathbf{M 1 - C u}$ at $875 \mathrm{~nm}$, in the absence and presence of amino acids, respectively; $c, d$ are constants. [substrate] is the concentration of the titrants (various amino acids ). Binding constant calculation for representative amino acids were shown in Appendix D, Figure S5.4.

Preparation of Human Plasma Samples. Pooled human plasma was thawed and deproteinized by adding $3 \mathrm{~mL}$ of acetone to $1 \mathrm{~mL}$ of plasma sample. After vortex-mixing for $10 \mathrm{~s}$, the mixture was centrifuged at $10,000 \mathrm{rpm}$ for $5 \mathrm{~min}$ (Mikro 22R refrigerated centrifuge, Hettich, Germany) to precipitate protein, and the supernatants obtained were concentrated by evaporation to ca. $1 / 3$ of the total volume under nitrogen flow. A human plasma with Cys solution $\left(1 \times 10^{-1} \mathrm{M}\right)$ was prepared by doping $1 \mathrm{~mL}$ of Cys $\left(2 \times 10^{-1} \mathrm{M}\right.$ in distilled water) into $1 \mathrm{~mL}$ of the deproteinized human plasma.

\section{Absorption and Fluorescence Titration of M1-Cu with Cys in Human Plasma}

Samples. A solution of $\mathrm{CuCl}_{2}$ in methanol $\left(40 \mu \mathrm{L}, 2 \times 10^{-1} \mathrm{M}\right)$ was added to $10 \mathrm{~mL}$ of M1 solution $\left(2 \times 10^{-5} \mathrm{M}\right)$ to form M1-Cu and quench the fluorescence. The M1 and M1$\mathrm{Cu}$ solution were placed in a quartz cuvette, respectively. Both the fluorescence and absorption spectra were then recorded. The solution of human plasma with Cys was introduced in portions $(2-35 \mu \mathrm{L})$ to $10 \mathrm{~mL}$ of $\mathbf{M 1}-\mathbf{C u}\left(\mathbf{M 1}=2 \times 10^{-5} \mathrm{M}\right)$ in DMF and the intensity changes in absorption and the fluorescence spectra were recorded $1 \mathrm{~min}$ after Cys addition at room temperature . 
Preparation of solutions of L-cystine, propylamine, ethanethiol, ethyl sulfide, homocysteine, L-glutathione and $\mathbf{N}$-acetylcysteine. $5 \mathrm{mmol}$ of each compound was dissolved in distilled water $(25.0 \mathrm{~mL})$ to afford a $2 \times 10^{-1} \mathrm{M}$ aqueous solution, except for L-cystine $\left(2 \times 10^{-3} \mathrm{M}\right)$.

Flourescence titration of M1-Cu with L-cystine, propylamine, ethanethiol and ethyl sufide. The solution of $\mathrm{Cu}^{2+}\left(2 \times 10^{-1} \mathrm{M}, 40 \mu \mathrm{L}\right)$ was added to $10 \mathrm{~mL}$ of $\mathrm{M1}\left(2 \times 10^{-5} \mathrm{M}\right.$ in DMF) solution to quench the fluorescence. The solutions of L-cystine, propylamine, ethanethiol or ethyl sulfide were introduced to the above M1-Cu solution in portions up to 40 equiv. of Cys $\left(2 \times 10^{-1} \mathrm{M}\right)$ and the fluorescence intensity changes were recorded at room temperature each time. 


\subsection{References}

(1) (a)Kramer, R. Angew. Chem., Int. Ed. 1998, 37, 772. (b)Chemosensors of Ion and Molecule Recognition; Desvergne, J. P., Czarnik, A. W., Eds.; NATO ASI Series; Kluwer Academic Publishers: Dordrecht, 1997. (c)De Silva, A. P.; Nimal Gunaratne, H. O.; Gunnlaugusson, T.; Huxley, A. J. M.; McCoy, C. P.; Rademacher, J. T.; Rice, T. E. Chem. Rev. 1997, 97, 1515. (d)Topics in Fluorescence Spectroscopy; Lakowicz, J. R., Ed.; Plenum Press: New York, 1994.

(2) (a)Lakowicz, J. R. Principle of Fluorescence Spectroscopy, Kluwer Academic / Plenum Publishers: New York, 1999. (b)Fluorescent Chemosensors for Ion and Molecule Recognition; Czarnik, A. W., Ed.; ACS Symposium Series 538, American Chemical Society: Washington, DC, 1993.

(3) (a)Akkaya, E. U.; Turkyilmaz, S. Tetrahedron Lett. 1997, 38, 4513. (b)Coskun, A.; Yilmaz, M. D.; Akkaya, E. U. Org. Lett. 2007, 9, 607. (c) Kiyose, K.; Kojima, H.; Urano, Y.; Nagano, T. J. Am. Chem. Soc. 2006, 128, 6548.

(4) Esposito, B. P.; Breuer, W.; Cabantchik, Z. I. Biochemical Society Transactions 2002, 30, 729.

(5) Breuer, W.; Epsztejn, S.; Milgram P.; Cabantchik, Z. I. Am. J. Physiol. Cell Physiol. 1995, 268, C1354.

(6) Zheng, Y.; Huo, Q.; Kele, P.; Andreopoulos, F. M.; Pham, S. M.; Leblanc, R. M. Org. Lett. 2001, 3, 3277.

(7) Bush, A. I. Curr. Opin. Chem. Biol. 2000, 4, 184.

(8) (a)Bhattacharya, S.; Thomas, M. Tetrahedron Lett. 2000, 41, 10313. (b)Torrado, A.; Walkup, G. K.; Imperiali, B. J. Am. Chem. Soc. 1998, 120, 609. (c)DeSantis, G.; Fabbrizzi, L.; Licchelli, M.; Mangano, C.; Sacchi, D.; Sardone, N. Inorg. Chim. Acta 1997, 257, 69.

(9) (a)Solomon, E. I.; Penfield, K. W.; Wilcox, D. E.; Structure Bonding; SpringerVerlag: New York, 1993. (b)Wallace, K. J.; Cordero, S. R.; Tan, C. P.; Lynch, V. M.; Anslyn, E.V. Sens. Actuators B 2007, 120, 362.

(10) (a)Bai, Y. W.; Song, N. H; Gao, J. P.; Sun, X; Wang, X. M.; Yu, G. M.; Wang, Z. Y. J. Am. Chem. Soc. 2005, 127, 2060. (b)Ashwell, G. J. Thin Solid Films 1990, 
186, 155. (c) Bell, N. A.; Broughton, R. A.; Brooks, J. S.; Jones, T. A.; Thorpe, S. C.; Ashwell, G. J. J. Chem. Soc., Chem.Commun. 1990, 325.

(11) Chow, C. F.; Lam, M. H.W.; Wong, W. Y. Inorg. Chem. 2004, 43, 8387.

(12) Voet, D.; Voet, J. G. Biochemistry, 2nd ed.; John Wiley \& Sons: New York, 1995.

(13) Shahrokhian, S. Anal. Chem. 2001, 73, 5972.

(14) (a)Klingman, J. G.; Choi, D. W. Neurology 1989, 39, 397. (b)Janaky, R.; Varga, V.; Hermann, A.; Saransaari, P.; Oja, S. S. Neurochem. Res. 2000, 25, 1397. (c) Gazit, V.; Coleman, R.; Weizman, A.; Katz, Y. Amino Acids 2004, 26, 163.

(15) (a)Vandeberg, P.; Johnson, D. C. Anal. Chem. 1993, 65, 2713. (b)Pelletier, S.; Lucy, C. A. Analyst 2004, 129, 710. (c)Sudeep, P. K.; Shibu Joseph, S. T.; Thomas, G. K. J. Am. Chem. Soc. 2005, 127, 6516. (d)Andrabi, S. M. Z.; Khan, Z. Colloid Polym. Sci. 2007, 285, 389. (e)Prasad, K.S.; Muthuraman, G.; Zen, J. M. Electroanalysis 2008, 20, 1167.

(16) (a)Nekrassova, O.; Lawrence, N. S.; Compton, R. G. Talanta 2003, 60, 1085. (b) Bai, Y. H.; Xu, J. J.; Chen, H. Y. Biosensors and Bioelectronics 2009, 24, 2985. (c) Li, S.; Dong, S. J. Biosensors and Bioelectronics 2009, 24, 1569. (d)Pastore, A.; Massoud, R.; Motti, C.; Russo, A.; Fucci, G.; Cortese, C.; Federici, G. Clin. Chem.1998, 44, 825.(e)Refsum, H.; Smith, A.D.; Ueland, P. M.; Nexo, E.; Clarke, R.; McPartlin, J.; Johnston, C.; Engbaek, F.; Schneede, J.; McPartlin, C.; Scott, J. M. Clin. Chem. 2004, 50, 3.

(17) (a)Chwatko, G.; Bald, E. Talanta 2000, 52, 509. (b)Shimada, K; Mitamura, K. J. Chromatogr. B 1994, 659, 227. (c)Pastore, A.; Massound, R.; Motti, C.; Russo, A. L.; Fucci, G.; Cortese, C.; Federici, G. Clin. Chem. 1998, 44, 825. (d)Winters, R. A.; Zukowski, J.; Ercal, N.; Matthews, R. H.; Spitz, D. R. Anal. Biochem. 1995, 227, 14. (e) Haugland, R. P. Handbook of Fluorescent Probes and Research Products, 9th ed.; Molecular Probes: Eugene, OR. 2002.

(18) (a)Chen, S. J.; Chang, H. T. Anal. Chem. 2004, 76, 3727. (b)Negi, D. P. S.; Chanu, T. I. Nanotechnology 2008, 19, 465503. (c)Ros-Lis, J.V.; Garci, B.; Jimenez, D.; Martinez-Manez, R.; Sancenon, F.; Soto, J.; Gonzalvo, F.; 
Valldecabres, M. C. J. Am. Chem. Soc.2004, 126, 4064. (d)Sreejith, S.; Divya, K.P.; Ajayaghosh, A. Angew. Chem. Int. Ed. 2008,47,7883.

(19) (a)Rusin, O.; Luce, N. N. S.; Agbaria, R. A.; Escobedo, J. O.; Jiang, S.; Warner, I. M.; Dawan, F. B.; Lian ,K.; Strongin, R. M. J. Am. Chem. Soc., 2004, 126, 438. (b)Wang, W. O.; Rusin, X.; Xu, K.; Kim, K.; Escobedo, J. O.; Fakayode, S. O.; Fletcher, K. A.; Lowry, M.; Schowalter, C. M.; Lawrence, C. M.; Fronczek, R. I.; Warner, M.; Strongin, R. M. J. Am. Chem. Soc. 2005, 127, 15949.

(20) (a)McQuade, D. T.; Hegedus, A. H.; Swager, T. M. J. Am. Chem. Soc. 2000, 122, 12389. (b)Rudzinski, C. M.; Hartmann, W. K.; Nocera, D. G. Coord. Chem. Rev. 1998, 171, 115. (c)Mortellaro, M. A.; Nocera, D. G. J. Am. Chem. Soc. 1996, 118,7414 .

(21) (a)Tanaka, F.; Mase, N.; Barbas, C. F. Chem. Commun. 2004,15,1762. (b) Tanaka, F.; Mase, N.; Barbas, C. F. J. Am. Chem. Soc., 2004, 126, 3692.

(22) Zhang, M.; Yu, M. X.; Li, F.Y.; Zhu, M. W.; Li, M. Y.; Gao, Y. H.; Liu, Z. Q.; Zhang, D. Q.; Yi, T.; Huang, C. H. J. Am. Chem. Soc. 2007, 129, 10322.

(23) Frausto da Silva, J. J. F.; Williams, R. J. P. The Biological Chemistry of Elements; Clarendon, Oxford, UK. 1991.

(24) Shortreed, M.; Kopelman, R.; Hoyland, B. Anal. Chem. 1996, 68, 1414.

(25) (a)Shang, L.; Yin, J.Y.; Li, J.; Jin, L.; Dong, S. J. Biosens. Bioelectron. 2009, 25, 269. (b)Li, H. L.; Fan, J. L.; Wang, J.Y.; Tian, M. Z.; Du, J. J.; Sun, S. G.; Sun, P. P.; Peng, X. J. Chem. Commun.2009, 5904.

(26) Everin, K.; Bergs, R.; Beck, W. Angew. Chem. Int. Ed.1998, 37, 1635.

(27) (a)Benesi, H. A.; Hildebrand, J. H. J. Am. Chem. Soc. 1949, 71, 2703. (b)Chow, C. F.; Lam, M. H. W.; Wong, W. Y. Inorg. Chem. 2004, 43, 8387.

(28) Refsum, H.; Ueland, P. M.; Nygard, O.; Vollset, S. E. Annu. Rev. Med. 1989, 49, 31 .

(29) Seshadri, S.; Beiser, A.; Selhub, J.; Jacques, P. F.; Rosenberg, I. H.; D’Agostino, R. B.; Wilson, P. W. F. N. Engl. J. Med. 2002, 346, 476.

(30) Yoshida, Y.; Ohiwa, Y.; Shimamura, M.; Izumi, T.; Yoshida, S.; Takahashi, K.; Miyairi, S.; Makimura, M.; Naganuma, A. J. Health Sci.2003, 49,527. 


\section{Contribution to Knowledge}

1. A new series of zwitterionic NLO chromphores, dicyanodimethane-based (FDCN) and their corresponding chromophore-polymers were designed and synthesized. Incorporation of bulk groups onto the central part of the FDCN chromophore was demonstrated as an effective strategy to minimize dipole-dipole molecular interaction.

2. Second series of zwitterionic chromophores, PpQDM with dipyridylium electron donors and strong tricyanoquino-dimethane electron acceptors were prepared and grafted onto host polyimides to yield a new series of NLO polyimides.

3. A large EO coefficient of $70 \mathrm{pm} / \mathrm{V}$ at the telecommunication wavelength of 1550 nm has been obtained with a 5 wt \% PpQDM-Ben doped PVP polymer using an in situ poling measurement technique.

4. A buffer layer (PEDOT: PSS/PVA) was first successfully introduced into the double layer device for zwitterionic NLO polymers, and improvement of the poling efficiency of NLO polymer systems was demonstrated.

5. PpQDMs is found to be NIR fluorescent with a large Stokes shift.

6. PpQDM-Met/copper complex was demonstrated to be the first high-contrast colorimetric and NIR fluorescence turn-on molecular probe for sensitive and selective detection of cysteine.

\section{Publications:}

1. Hao, W.; Wang, Z. Y. "Synthesis, crystal structures, and photophysical properties of second-order nonlinear optical zwitterionic 1-substituted-4-pyridiniumfluorophenyl-dicyanomethanide chromophores" (In preparation.) 
2. Hao, W.; Wang, Z. Y. "1-hexyl-4-pyridinium-fluorophenyl-dicyanomethanide" (To be submitted to Acta crystollographic)

3. Ma, J.; Chiniforooshan, Y.; Chen, J. H.; Bock, W. J.; Hao, W.; Wang, Z. Y. "Recapturing Lost Evanescent Power by Tuning Endface-TIR-Capable Tunneling Modes at a Roughened Fiber Endface" (Submittited to Optical Letter July, 2011)

4. Hao, W.; McBride, A.; McBride, S.; Wang, Z. Y. "Colorimetric and near-infrared fluorescence turn-on molecular probe for selective detection of cysteine in human plasma" J. Mater.Chem.2011, 21, 1040.

5. Ma, J.; Bock, W. J.; Wang, Z. Y.; Hao, W.; MacKinnon, S. M. "Investigation of a large-core photonic crystal fiber sensor for enhancement of fluorescent light collection of polymer membrane" Proceedings of SPIE-The International Society for Optical Engineering 2005, 5970 (Pt. 1, Photonic Applications in Devices and Communication Systems), 597006/1-597006/6

6. Ma, J.; Bock, W. J.; Wang, Z. Y.; Hao, W.; MacKinnon, S. M. "Fiber-optic membrane fluorescent sensor based on photonic crystal fiber with a glass rod in the fiber end" IEEE Sensors 2005, [IEEE Conference on Sensors], 4th, Irvine, CA, United States, Oct. 30-Nov. 3, 2005 2005, 2, 1096-1099.

7. Ma, J.; Bock, W. J.; Wang, Z. Y.; Hao, W. MacKinnon, S. M. "Towards optimum sample probe spectrometer system design by adjusting receiving fiber end face position and probe-membrane sample separation" Optics express $\mathbf{2 0 0 5}$, 13,9492 . 


\section{Conference presentations}

1. Hao, W.; Wang, Z. Y. "Development of Zwitterionic Chromophores and Polymers for Electro-Optic Application" $34^{\text {th }}$ Canadian High Polymer Forum, Hôtel Mont Gabriel, Ste-Adèle, Québec, Aug. 18-20, 2010 (Oral Presentation)

2. Wang, Z. Y.; Gao, J. P.; Zhang, J.; Tang, H.; LeClair, G.; Xun, S.; Xiong, Y.; Hao, W.; Volland, S. "Infrared Organic Materials for Optoelectronic and Sensor Applications" $32^{\text {nd }}$ Canadian High Polymer Forum, Aylmer, Quebec, Aug.19-21, 2005 (Oral Presentation)

3. Song, N. H.; Bai, Y. W.; Xiong, Y.; Hao, W.; Volland, S.; Wang, X.; Sun, X.; Yu, G.; Men, L.; Gao, J. P.; Wang, Z. Y. “A New Approach to Highly ElectroOptically Active Polymers Using Cross-Linkable, Hyperbranched Chromophore Containing Oligomers as Macromolecular Dopants" $88^{\text {th }}$ Canadian Chemistry Conference and Exhibition, Saskatoon, SK, Canada, May 28-June 1, 2005 (Award Presentation) 


\section{Appendix A X-ray data for FDCN}

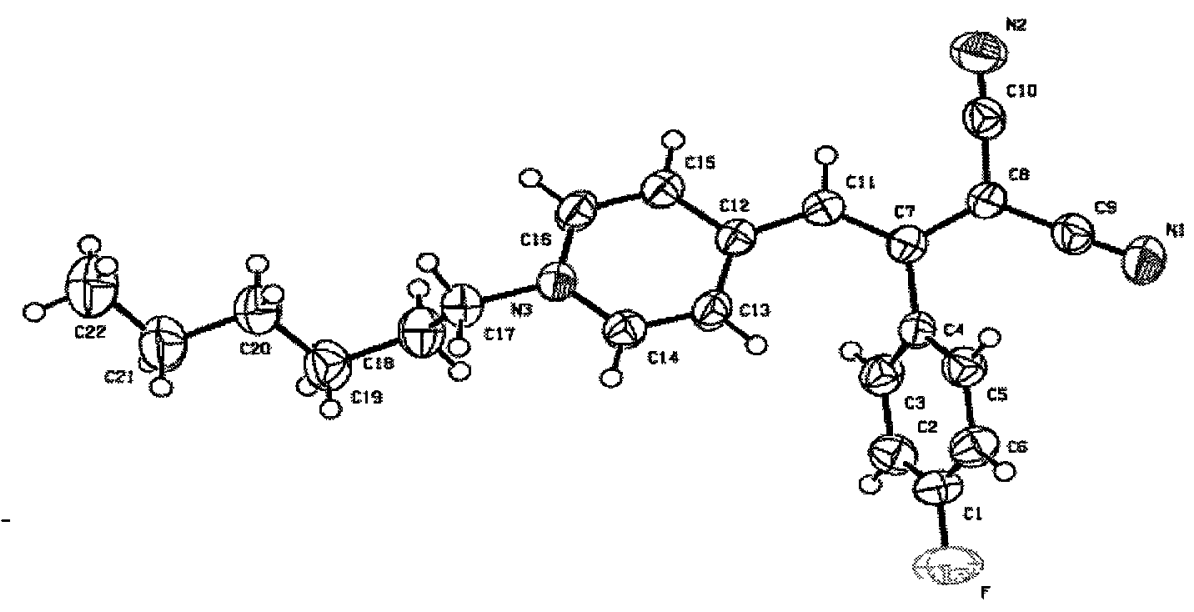

Table 1. Crystal data and structure refinement for FDCN

\begin{tabular}{ll}
\hline Identification code & $\mathrm{P} 237$ \\
Empirical formula & $\mathrm{C} 22 \mathrm{H} 22 \mathrm{~F} \mathrm{~N} 3$ \\
Formula weight & 347.43 \\
Temperature & $293(2) \mathrm{K}$ \\
Wavelength & $0.71073 \AA$ \\
Crystal system & Monoclinic \\
Space group & $\mathrm{P} 2(1) / \mathrm{c}$ \\
Unit cell dimensions & $\mathrm{a}=10.485(3) \AA \quad \mathrm{b}=8.809(2) \AA$ \\
& $\mathrm{c}=21.313(5) \AA$ \\
& $\alpha=90.00^{\circ} \quad \beta=100.628(4)^{\circ} \quad \gamma=90.00^{\circ}$ \\
Volume & $1934.7(8) \AA^{3}$ \\
$\mathrm{Z}$ & 4 \\
Density (calculated) & $1.193 \mathrm{Mg} / \mathrm{m}^{3}$ \\
Absorption coefficient & $0.08 \mathrm{~mm}^{-1}$ \\
F(000) & 736.0 \\
Crystalsize & $0.437 \times 0.217 \times 0.056 \mathrm{~mm}^{3}$ \\
Theta range for data collection & 1.94 to $28.68^{\circ}$ \\
Index ranges & $-13=<\mathrm{h}=<10$ \\
& $-11=<\mathrm{k}=<11$ \\
Reflections collected & $-26=<1=<27$ \\
Independent reflections & 11920 \\
Completeness to theta $=28.68^{\circ}$ & 4589 \\
Absorption correction & $92 \%$ \\
Max, and Min. transmission & Semi-empirical from equivalents \\
Refinement method & 0.9956 and 0.9667 \\
Data/restrains/parameters & Full-matrix least-squares on $\mathrm{F}^{2}$ \\
Goodness-of-fit on $\mathrm{F}^{2}$ & $4589 / 0 / 236$ \\
Final R indices [l>2sigma(I)] & 0.991 \\
R indices (all data) & $\mathrm{R}=0.0505$ \\
Largest diff. peak and hole & $\mathrm{R} 1=0.1084, \mathrm{wR} 2=0.0902$ \\
& 0.153 and -0.165 e. $\AA^{-3}$ \\
\hline
\end{tabular}


Table 2. Atomic coordinates $\left(\times 10^{4}\right)$ and equivalent isotropic displacement parameters $\left(\AA^{2} \times 10^{3}\right)$ for FDCN. $U(e q)$ is defined as one third of the trace of the orthogonalized $U^{i j}$ tensor

\begin{tabular}{|c|c|c|c|c|}
\hline Atom & $\mathrm{x}$ & $y$ & $\mathrm{z}$ & $\mathrm{U}^{\mathrm{ij}}(\mathrm{eq})$ \\
\hline $\mathrm{F}$ & $95(1)$ & $3228(1)$ & $1471(1)$ & $89(1)$ \\
\hline $\mathrm{N} 1$ & $5246(2)$ & $19(2)$ & $2192(1)$ & $76(1)$ \\
\hline $\mathrm{N} 2$ & $7935(2)$ & $489(2)$ & $868(1)$ & $70(1)$ \\
\hline N3 & $3170(1)$ & $4945(2)$ & $-1432(1)$ & $44(1)$ \\
\hline $\mathrm{Cl}$ & $1227(2)$ & $2873(2)$ & $1278(1)$ & $54(1)$ \\
\hline $\mathrm{C} 2$ & $1180(2)$ & $1996(2)$ & $752(1)$ & $54(1)$ \\
\hline $\mathrm{C} 3$ & $2329(2)$ & $1660(2)$ & $552(1)$ & $46(1)$ \\
\hline $\mathrm{C} 4$ & $3509(2)$ & $2197(2)$ & $886(1)$ & $38(1)$ \\
\hline $\mathrm{C} 5$ & $3499(2)$ & $3064(2)$ & $1427(1)$ & $45(1)$ \\
\hline $\mathrm{C} 6$ & $2351(2)$ & $3428(2)$ & $1626(1)$ & $54(1)$ \\
\hline $\mathrm{C} 7$ & $4744(2)$ & $1794(2)$ & $678(1)$ & $38(1)$ \\
\hline $\mathrm{C} 8$ & $5674(2)$ & $1016(2)$ & $1123(1)$ & $41(1)$ \\
\hline C9 & $5426(2)$ & $482(2)$ & $1713(1)$ & $50(1)$ \\
\hline $\mathrm{C} 10$ & $6925(2)$ & $696(2)$ & $989(1)$ & $48(1)$ \\
\hline $\mathrm{C} 11$ & $4969(2)$ & $2106(2)$ & $71(1)$ & $42(1)$ \\
\hline $\mathrm{C} 12$ & $4302(2)$ & $3065(2)$ & $-414(1)$ & $39(1)$ \\
\hline $\mathrm{C} 13$ & $3342(2)$ & $4151(2)$ & $-359(1)$ & $47(1)$ \\
\hline $\mathrm{C} 14$ & $2814(2)$ & $5041(2)$ & $-856(1)$ & $481(1)$ \\
\hline $\mathrm{C} 15$ & $4656(2)$ & $3023(2)$ & $-1022(1)$ & $47(1)$ \\
\hline $\mathrm{C} 16$ & 4093(2) & $3923(2)$ & $-1507(1)$ & $49(1)$ \\
\hline $\mathrm{C} 17$ & $2554(2)$ & $5899(2)$ & $-1975(1)$ & $49(1)$ \\
\hline $\mathrm{C} 18$ & $1528(2)$ & $5046(2)$ & $-2425(1)$ & $59(1)$ \\
\hline C19 & $772(2)$ & $6054(2)$ & $-2945(1)$ & $67(1)$ \\
\hline $\mathrm{C} 20$ & $1555(2)$ & $6747(2)$ & $-3398(1)$ & $62(1)$ \\
\hline $\mathrm{C} 21$ & $729(2)$ & $7591(2)$ & $-3947(1)$ & $75(1)$ \\
\hline $\mathrm{C} 22$ & $1493(2)$ & $8187(2)$ & $-4426(1)$ & $872(1)$ \\
\hline
\end{tabular}


Table 3. Bond lengths $[\AA]$ and angles $\left[^{\circ}\right]$ for FDCN

\begin{tabular}{|c|c|}
\hline$F-C(1)$ & $1.362(2)$ \\
\hline$N(1)-C(9)$ & $1.147(2)$ \\
\hline$N(2)-C(10)$ & $1.151(2)$ \\
\hline$N(3)-C(14)$ & $1.349(2)$ \\
\hline$N(3)-C(16)$ & $1.352(2)$ \\
\hline$N(3)-C(17)$ & $1.480(2)$ \\
\hline$C(1)-C(2)$ & $1.355(2)$ \\
\hline$C(1)-C(6)$ & $1.363(2)$ \\
\hline$C(2)-C(3)$ & $1.382(2)$ \\
\hline$C(3)-C(4)$ & $1.392(2)$ \\
\hline$C(4)-C(5)$ & $1.386(2)$ \\
\hline$C(4)-C(7)$ & $1.487(2)$ \\
\hline$C(5)-C(6)$ & $1.386(2)$ \\
\hline$C(7)-C(11)$ & $1.384(2)$ \\
\hline$C(7)-C(8)$ & $1.408(2)$ \\
\hline$C(8)-C(9)$ & $1.411(2)$ \\
\hline$C(8)-C(10)$ & $1.420(2)$ \\
\hline$C(11)-C(12)$ & $1.416(2)$ \\
\hline$C(12)-C(13)$ & $1.409(2)$ \\
\hline$C(12)-C(15)$ & $1.413(2)$ \\
\hline$C(13)-C(14)$ & $1.352(2)$ \\
\hline$C(15)-C(16)$ & $1.349(2)$ \\
\hline$C(17)-C(18)$ & $1.504(2)$ \\
\hline$C(18)-C(19)$ & $1.525(2)$ \\
\hline$C(19)-C(20)$ & $1.508(2)$ \\
\hline$C(20)-C(21)$ & $1.516(2)$ \\
\hline$C(21)-C(22)$ & $1.503(2)$ \\
\hline$C(14)-N(3)-C(16)$ & $118.3(1)$ \\
\hline$C(14)-N(3)-C(17)$ & $121.44(14)$ \\
\hline$C(16)-N(3)-C(17)$ & $120.25(14)$ \\
\hline$C(2)-C(1)-F$ & $118.76(17)$ \\
\hline$C(2)-C(1)-C(6)$ & $123.4(2)$ \\
\hline$F-C(1)-C(6)$ & $117.83(18)$ \\
\hline$C(1)-C(2)-C(3)$ & $118.52(16)$ \\
\hline$C(2)-C(3)-C(4)$ & $120.83(16)$ \\
\hline$C(5)-C(4)-C(3)$ & $118.1(2)$ \\
\hline$C(5)-C(4)-C(7)$ & $121.38(14)$ \\
\hline$C(3)-C(4)-C(7)$ & $120.48(15)$ \\
\hline$C(6)-C(5)-C(4)$ & $121.54(16)$ \\
\hline$C(1)-C(6)-C(5)$ & $117.59(17)$ \\
\hline$C(11)-C(7)-C(8)$ & $120.66(15)$ \\
\hline$C(11)-C(7)-C(4)$ & $122.66(14)$ \\
\hline$C(8)-C(7)-C(4)$ & $116.63(14)$ \\
\hline$C(9)-C(8)-C(7)$ & $123.0(2)$ \\
\hline$C(7)-C(8)-C(10)$ & $120.83(14)$ \\
\hline$C(9)-C(8)-C(10)$ & $116.16(14)$ \\
\hline$N(1)-C(9)-C(8)$ & $178.3(2)$ \\
\hline$N(2)-C(10)-C(8)$ & $177.25(20)$ \\
\hline$C(7)-C(11)-C(12)$ & $130.85(15)$ \\
\hline$C(13)-C(12)-C(15)$ & $114.0(2)$ \\
\hline$C(13)-C(12)-C(11)$ & $127.46(15)$ \\
\hline$C(15)-C(12)-C(11)$ & $118.45(15)$ \\
\hline
\end{tabular}




\begin{tabular}{|l|l|}
\hline$C(14)-C(13)-C(12)$ & $121.64(15)$ \\
$N(3)-C(14)-C(13)$ & $122.25(16)$ \\
$C(16)-C(15)-C(12)$ & $122.34(16)$ \\
$C(15)-C(16)-N(3)$ & $121.46(15)$ \\
$N(3)-C(17)-C(18)$ & $111.85(14)$ \\
$C(17)-C(18)-C(19)$ & $112.78(15)$ \\
C (20) - C (19) - C (18) & $115.47(16)$ \\
C (19) - C (20) - C (21) & $113.02(16)$ \\
C (22) - C (21) - C (20) & $113.23(17)$ \\
\hline
\end{tabular}

Symmetry transformations used to generate equivalent atoms

Table 4. Anisotropic displacement parameters $\left(\AA^{2}\right)$ for FDCN. Anisotropic displacement factor exponent takes the form: $-2 \pi^{2}\left[h^{2} a^{* 2} U^{11}+\ldots+2 h k a * b * U^{12}\right]$

\begin{tabular}{|c|c|c|c|c|c|c|}
\hline Atom & $\mathrm{U}^{11}$ & $\mathrm{U}^{22}$ & $\mathrm{U}^{33}$ & $\mathrm{U}^{23}$ & $U^{13}$ & $U^{12}$ \\
\hline $\bar{F}$ & $0.0578(8)$ & $0.1138(11)$ & $0.1017(9)$ & $-0.0069(8)$ & $0.0344(7)$ & $0.0152(7)$ \\
\hline N1 & $0.0951(14)$ & $0.0947(14)$ & $0.0430(10)$ & $0.0155(10)$ & $0.0217(9)$ & $0.0315(10)$ \\
\hline $\mathrm{N} 2$ & $0.0523(11)$ & $0.0930(14)$ & $0.0629(11)$ & $-0.0080(9)$ & $0.0075(8)$ & $0.0117(9)$ \\
\hline N3 & $0.0491(9)$ & $0.49451(15)$ & $0.0354(8)$ & $0.0046(7)$ & $0.0079(6)$ & $0.0016(7)$ \\
\hline $\mathrm{C} 1$ & $0.0470(12)$ & $0.2873(2)$ & $0.0576(12)$ & $0.0065(11)$ & $0.0220(10)$ & $0.0102(10)$ \\
\hline $\mathrm{C} 2$ & $0.0410(11)$ & $0.0649(13)$ & $0.0527(12)$ & $0.0034(10)$ & $0.0028(9)$ & $-0.0037(9)$ \\
\hline $\mathrm{C} 3$ & $0.0487(12)$ & $0.0532(12)$ & $0.0366(10)$ & $-0.0040(8)$ & $0.0057(8)$ & $-0.0028(9)$ \\
\hline $\mathrm{C} 4$ & $0.0422(11)$ & $0.0409(10)$ & $0.0303(9)$ & $0.0037(8)$ & $0.0066(8)$ & $0.0008(8)$ \\
\hline $\mathrm{C} 5$ & $0.0457(11)$ & $0.0470(11)$ & $0.0410(10)$ & $-0.0027(9)$ & $0.0044(8)$ & $-0.0001(8)$ \\
\hline $\mathrm{C} 6$ & $0.0625(13)$ & $0.0565(13)$ & $0.0451(11)$ & $-0.0083(9)$ & $0.0163(10)$ & $0.0070(10)$ \\
\hline $\mathrm{C} 7$ & $0.0417(10)$ & $0.0373(10)$ & $0.0343(9)$ & $-0.0034(8)$ & $0.0064(8)$ & $-0.0033(8)$ \\
\hline $\mathrm{C} 8$ & $0.0418(11)$ & $0.0490(11)$ & $0.0320(10)$ & $-0.0016(8)$ & $0.0077(8)$ & $0.0034(8)$ \\
\hline C9 & $0.0548(12)$ & $0.0588(13)$ & $0.17131(8)$ & $-0.0011(9)$ & $0.0048(9)$ & $0.0132(9)$ \\
\hline $\mathrm{ClO}$ & $0.0525(12)$ & $0.0522(12)$ & $0.0351(10)$ & $-0.0032(8)$ & $0.0001(9)$ & $0.0033(9)$ \\
\hline C11 & $0.0431(10)$ & $0.0463(11)$ & $0.0383(10)$ & $0.0001(8)$ & $0.0107(8)$ & $0.0058(8)$ \\
\hline $\mathrm{C} 12$ & $0.0402(10)$ & $0.0413(10)$ & $0.0350(9)$ & $-0.0011(8)$ & $0.0079(7)$ & $-0.0036(8)$ \\
\hline $\mathrm{C} 13$ & $0.0586(12)$ & $0.0506(11)$ & $0.0327(10)$ & $0.0016(9)$ & $0.0141(8)$ & $0.0059(9)$ \\
\hline $\mathrm{C} 14$ & $0.0548(12)$ & $0.0508(11)$ & $0.0407(11)$ & $-0.0004(9)$ & $0.0141(8)$ & $0.0079(9)$ \\
\hline $\mathrm{C} 15$ & $0.0522(11)$ & $0.0492(12)$ & $0.0415(10)$ & $0.0048(9)$ & $0.0157(8)$ & $0.0082(8)$ \\
\hline $\mathrm{C} 16$ & $0.0604(13)$ & $0.0566(12)$ & $0.0356(10)$ & $0.0024(9)$ & $0.0174(9)$ & $0.0019(10)$ \\
\hline $\mathrm{C} 17$ & $0.0550(12)$ & $0.0479(11)$ & $0.0416(10)$ & $0.0066(9)$ & $0.0058(8)$ & $0.0011(9)$ \\
\hline $\mathrm{C} 18$ & $0.0684(13)$ & $0.0556(13)$ & $0.24246(8)$ & $0.0008(10)$ & $0.0015(9)$ & $0.0088(10)$ \\
\hline $\mathrm{C} 19$ & $0.0627(14)$ & $0.0831(16)$ & $0.0495(12)$ & $0.0046(11)$ & $0.0045(10)$ & $0.0051(11)$ \\
\hline
\end{tabular}




\begin{tabular}{|lllllll}
\hline $\mathrm{C} 20$ & $0.0696(14)$ & $0.0663(14)$ & $0.0469(12)$ & $0.0001(10)$ & $0.0028(10)$ & $0.0007(11)$ \\
$\mathrm{C} 21$ & $0.0811(16)$ & $0.7591(2)$ & $0.0518(13)$ & $0.0126(12)$ & $0.0001(11)$ & $0.0052(12)$ \\
$\mathrm{C} 22$ & $0.1042(19)$ & $0.0917(19)$ & $0.0603(14)$ & $0.0088(13)$ & $0.0011(12)$ & $0.0128(14)$
\end{tabular}

Table 5. Hydrogencoordinates $\left(\times 10^{4}\right)$ and isotropic displacement parameters $\left(\AA^{2} \times 10^{3}\right)$ for FDCN

\begin{tabular}{lllll}
\hline Atom & $\mathrm{x}$ & $\mathrm{y}$ & $\mathrm{z}$ & $\mathrm{U}^{\mathrm{y}}(\mathrm{eq})$ \\
$\mathrm{H}(2)$ & 393 & 1629 & 530 & 54 \\
$\mathrm{H}(3)$ & 2313 & 1067 & 189 & 46 \\
$\mathrm{H}(5)$ & 4281 & 3411 & 1662 & 45 \\
$\mathrm{H}(6)$ & 2348 & 4029 & 1985 & 54 \\
$\mathrm{H}(11)$ & 5678 & 1601 & -36 & 42 \\
$\mathrm{H}(13)$ & 3066 & 4259 & 29 & 47 \\
$\mathrm{H}(14)$ & 2182 & 5742 & 800 & 48 \\
$\mathrm{H}(15)$ & 5301 & 2351 & -1091 & 47 \\
$\mathrm{H}(16)$ & 4344 & 3839 & -1902 & 50 \\
$\mathrm{H}(17 \mathrm{~A})$ & 3213 & 6252 & -2206 & 49 \\
$\mathrm{H}(17 \mathrm{~B})$ & 2166 & 6783 & -1814 & 49 \\
$\mathrm{H}(18 \mathrm{~A})$ & 928 & 4590 & -2183 & 59 \\
$\mathrm{H}(18 \mathrm{~B})$ & 1936 & 4232 & -2622 & 59 \\
$\mathrm{H}(19 \mathrm{~A})$ & 78 & 5457 & -3192 & 67 \\
$\mathrm{H}(19 \mathrm{~B})$ & 373 & 6868 & -2743 & 67 \\
$\mathrm{H}(20 \mathrm{~A})$ & 2030 & 5949 & -3569 & 62 \\
$\mathrm{H}(20 \mathrm{~B})$ & 2185 & 7444 & -3163 & 62 \\
$\mathrm{H}(21 \mathrm{~A})$ & 58 & 6915 & -4162 & 74 \\
$\mathrm{H}(21 \mathrm{~B})$ & 305 & 8435 & -3777 & 74 \\
$\mathrm{H}(22 \mathrm{~A})$ & 2175 & 8834 & -4214 & 87 \\
$\mathrm{H}(22 \mathrm{~B})$ & 929 & 8755 & -4748 & 87 \\
$\mathrm{H}(22 \mathrm{C})$ & 1861 & 7352 & -4620 & 87 \\
\hline
\end{tabular}

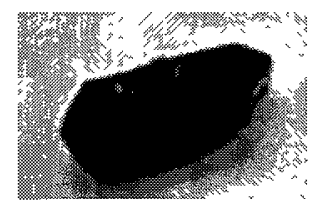


Appendix B Spectra of Synthesized Products of Chapter 2 and 3

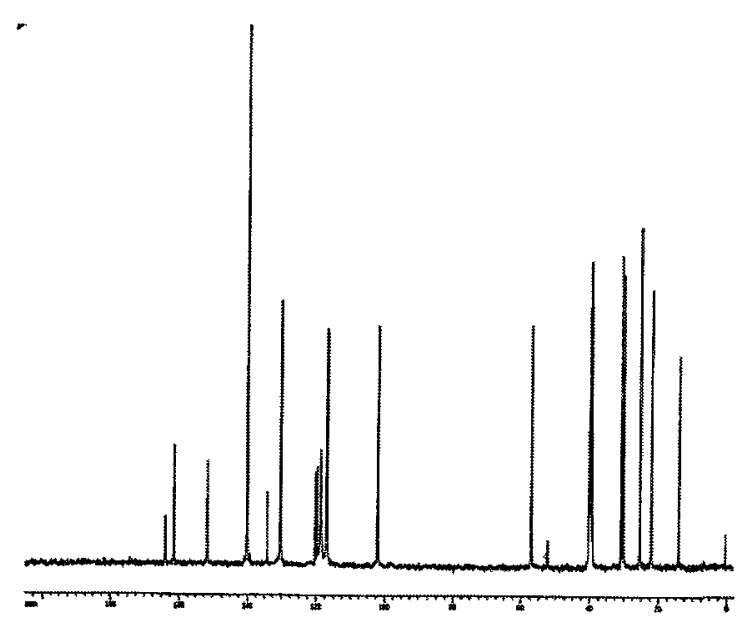

Figure S2.1. ${ }^{13} \mathrm{C}$ NMR of FDCN.
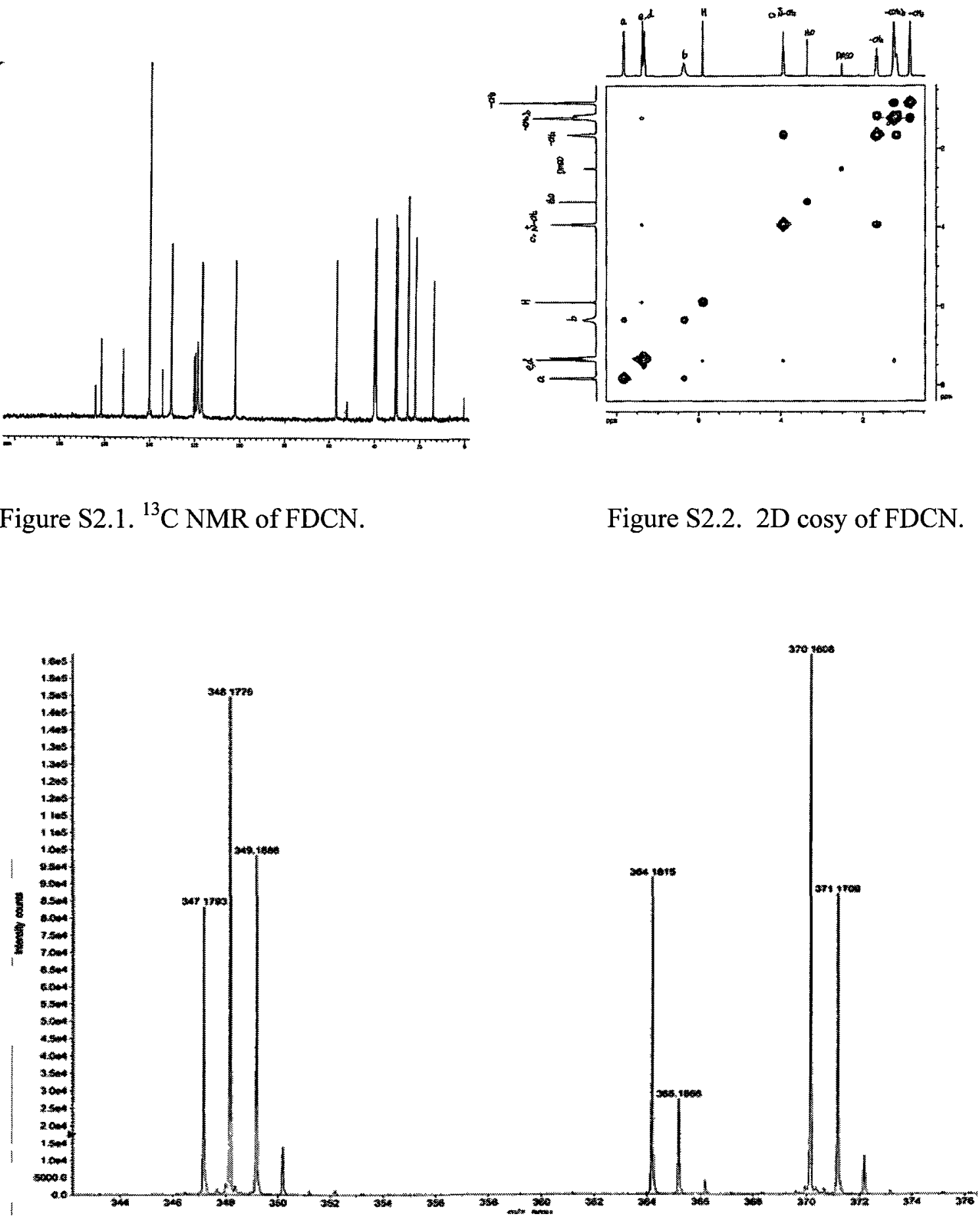

Figure S2.3. MS spectrum of FDCN. 

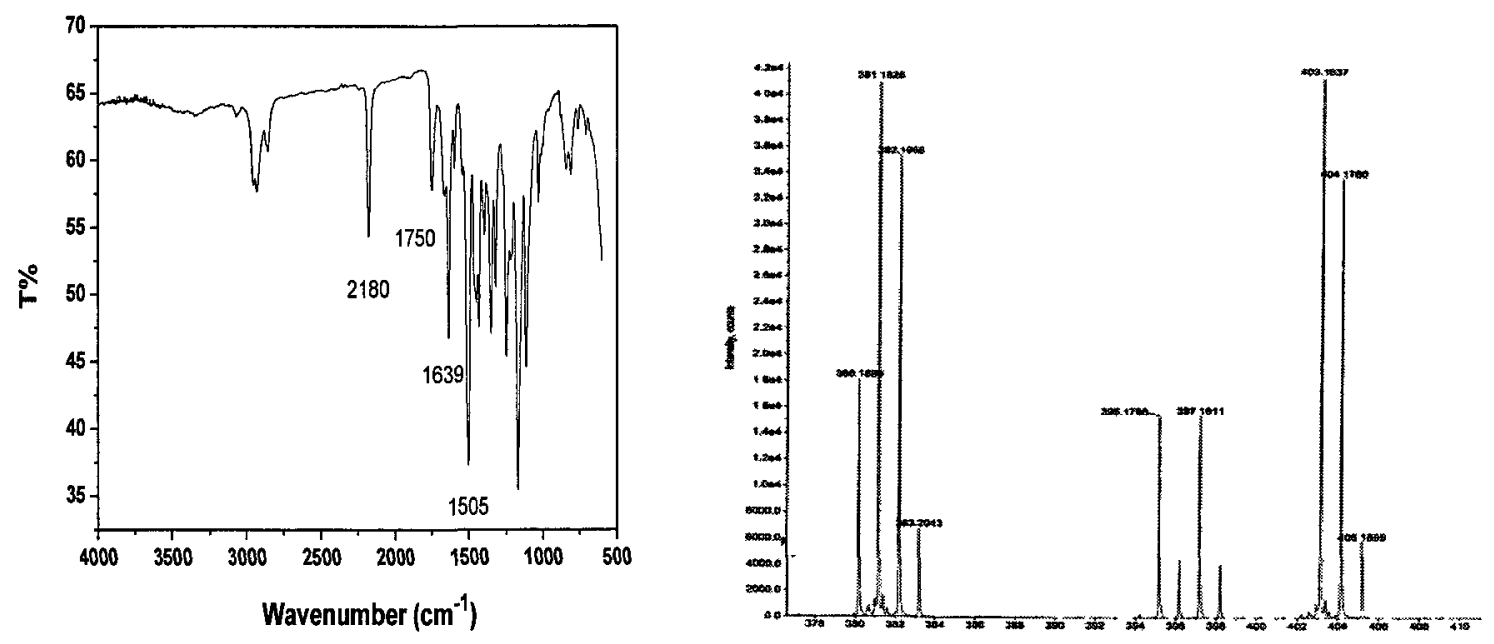

Figure S2.4. IR spectrum of FCNE (KBr pellet). Figure S2.5. MS spectrum of FCNE.
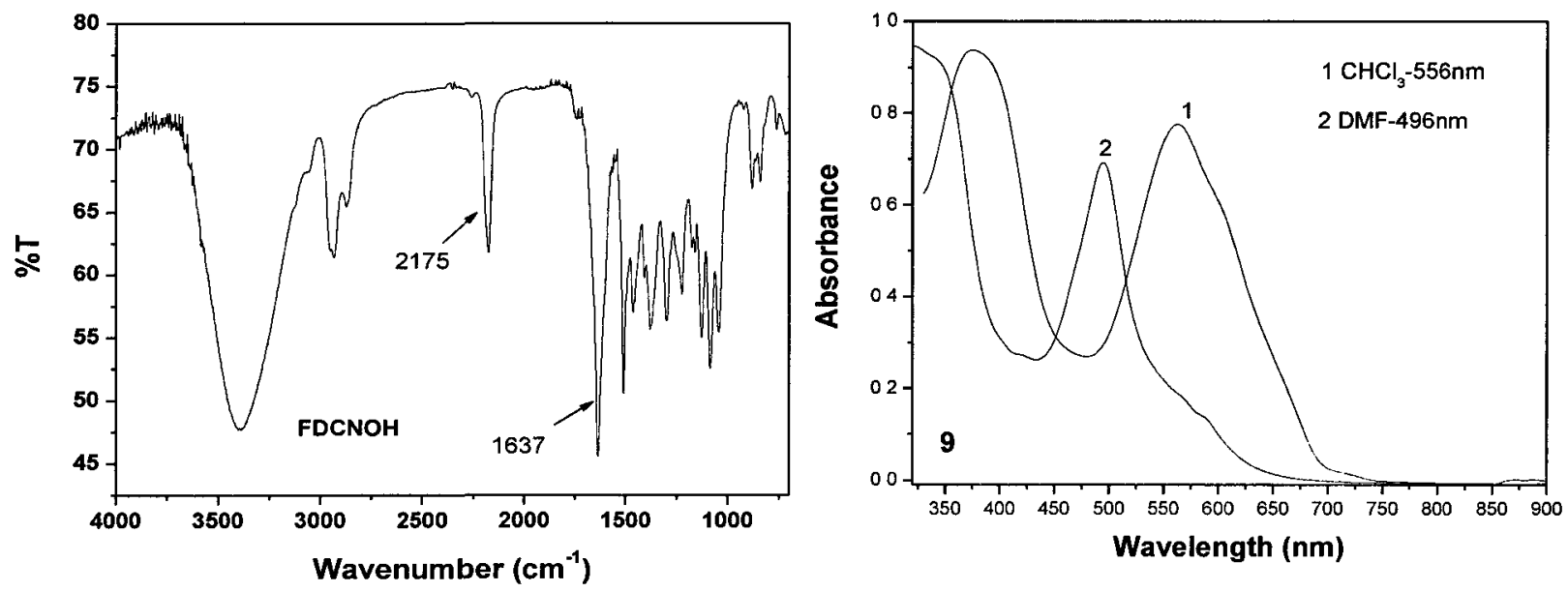

Figure S2.6. IR spectrum of FDCNOH (KBr pellet). Figure S2.7. UV spectra of $\mathbf{A} 2$ in DMF and chloroform. 


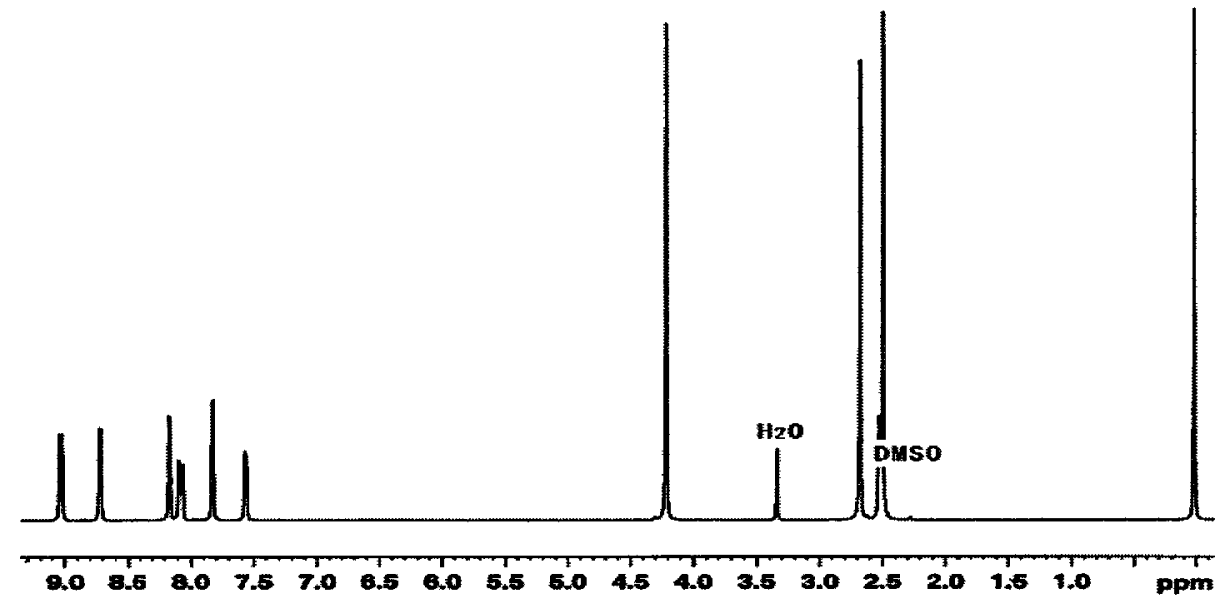

Figure S2.8. ${ }^{1} \mathrm{H}$ NMR of 1,4,4'-trimethyl-2, 2'-dipyridylium iodide.

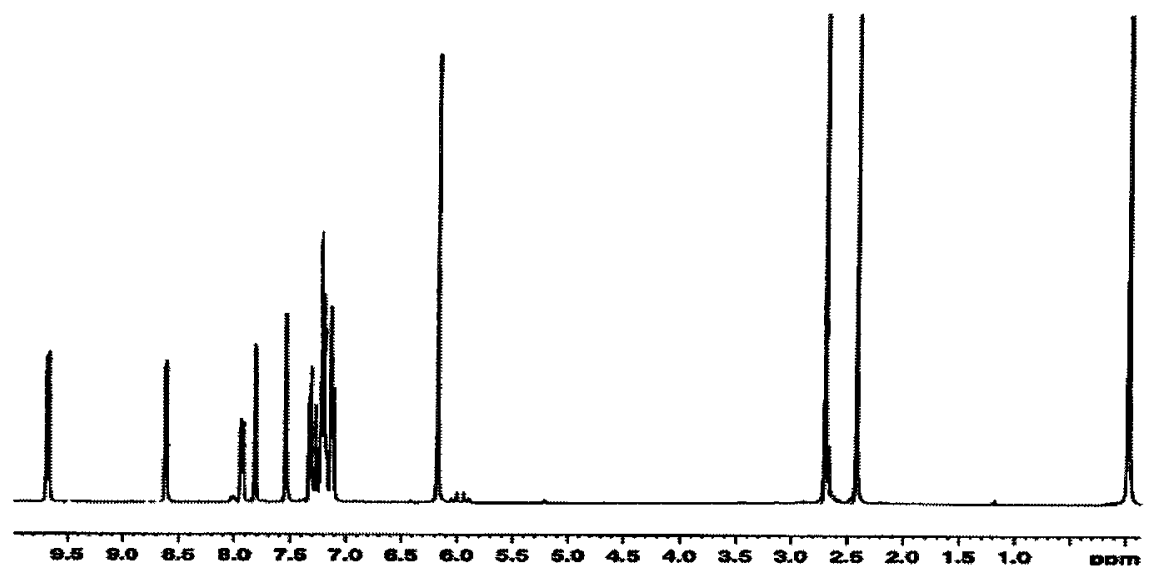

Figure S2.9. ${ }^{1} \mathrm{H}$ NMR of 1-benzyl -4,4'-trimethyl-2, 2'-dipyridylium bromide.

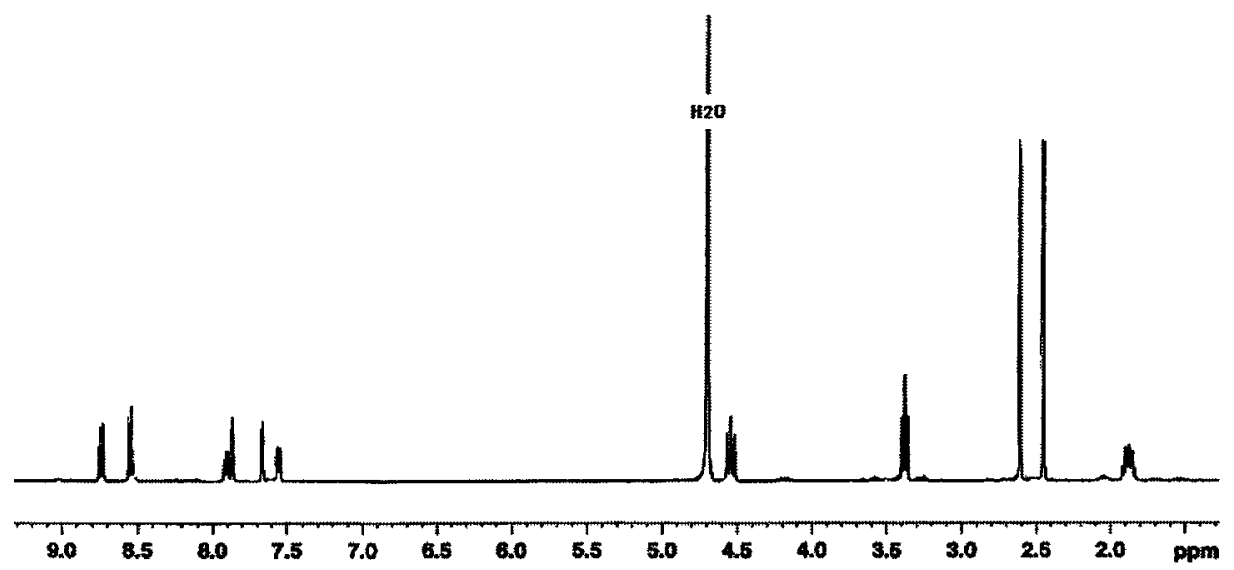

Figure S2.10. ${ }^{1} \mathrm{HNMR}$ of 1-hydroxypropyl -4, 4'-trimethyl-2, 2'-dipyridylium bromide. 

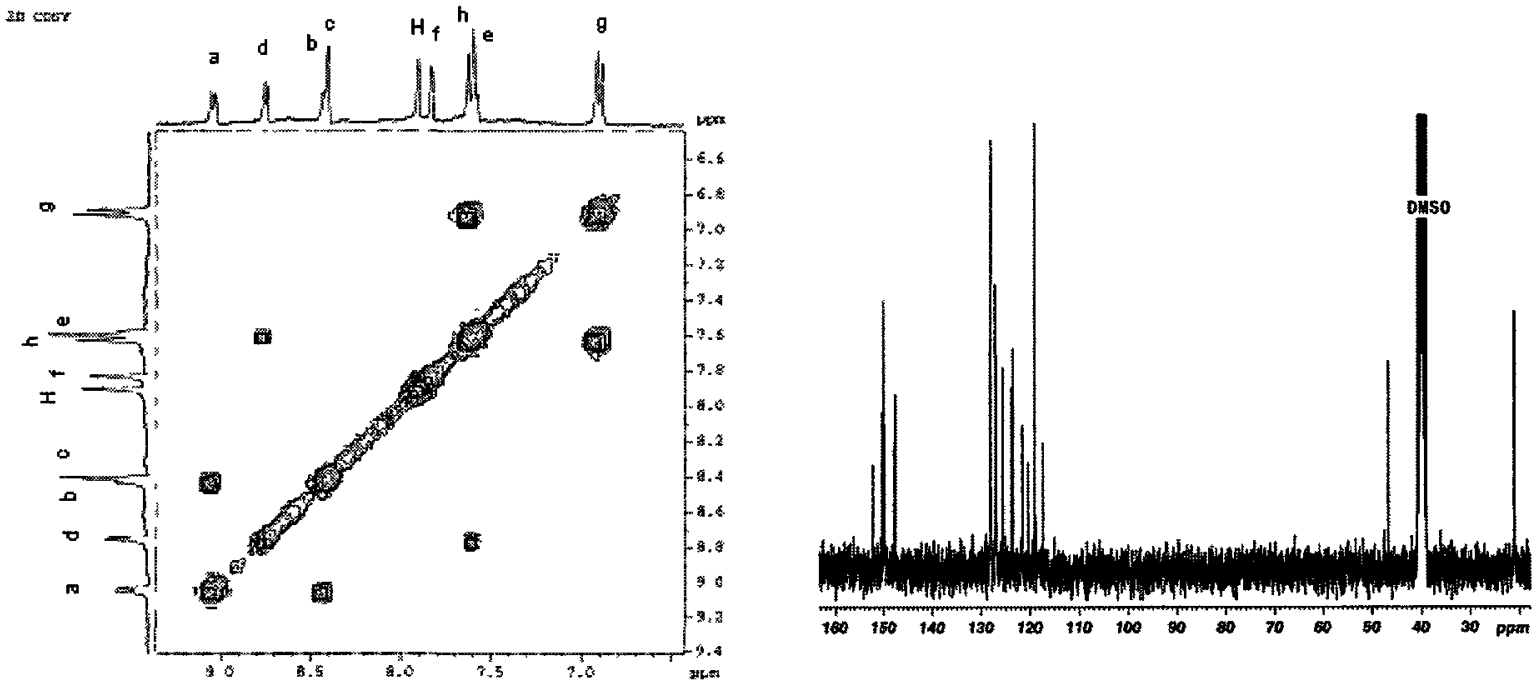

Figure S2.11. ${ }^{1} \mathrm{H}$ NMR (2D COSY) spectrum of aromatic protons of PpQDM-Met in DMSO- $_{6}$ (left) and Figure S2.12. ${ }^{13} \mathrm{C}$ NMR of PpQDM-Met (right).

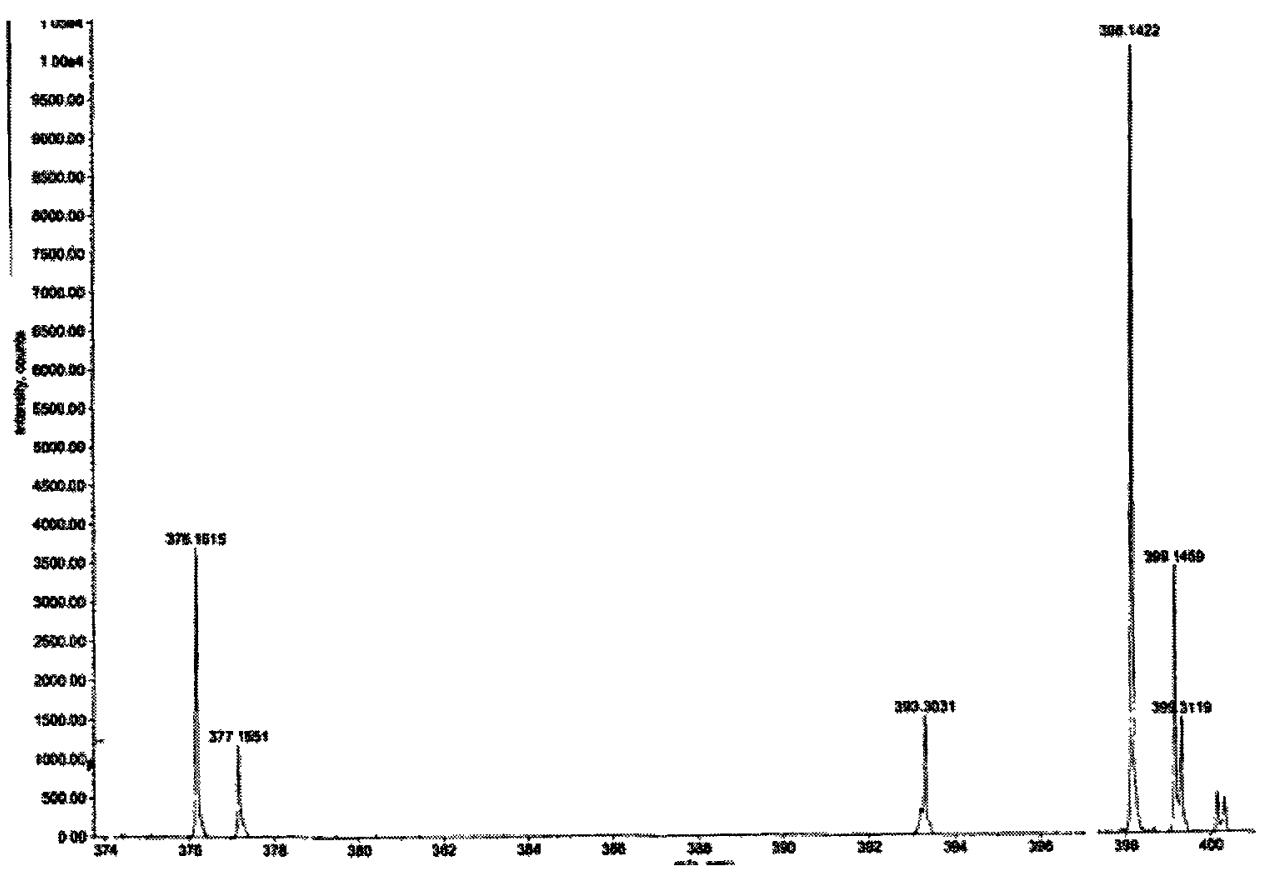

Figure S2.13. ESI mass spectrum of PpQDM-Met. 


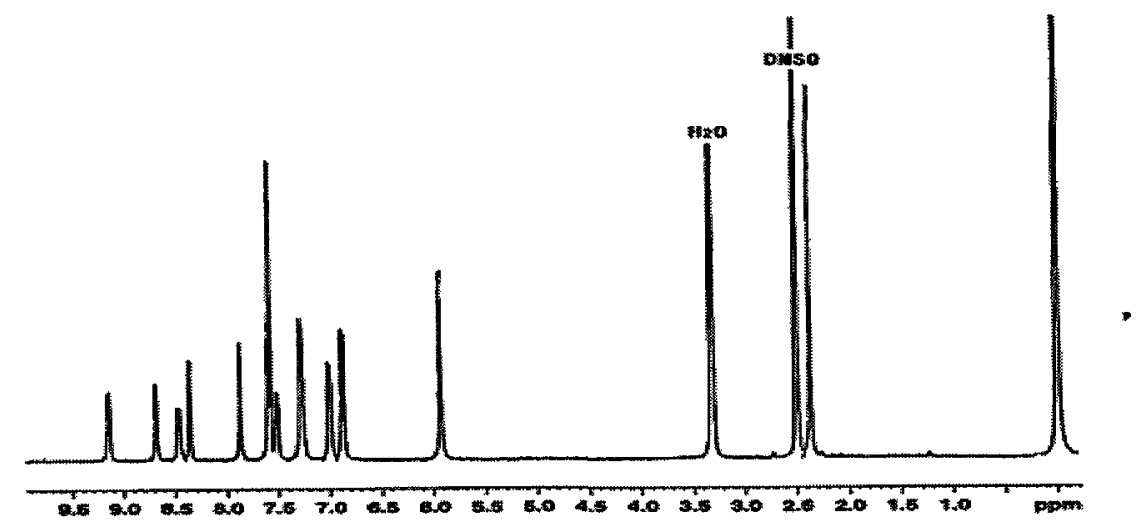

Figure S2.14. ${ }^{1} \mathrm{HNMR}$ of PpQDM-Ben in DMSO-d ${ }_{6}$.
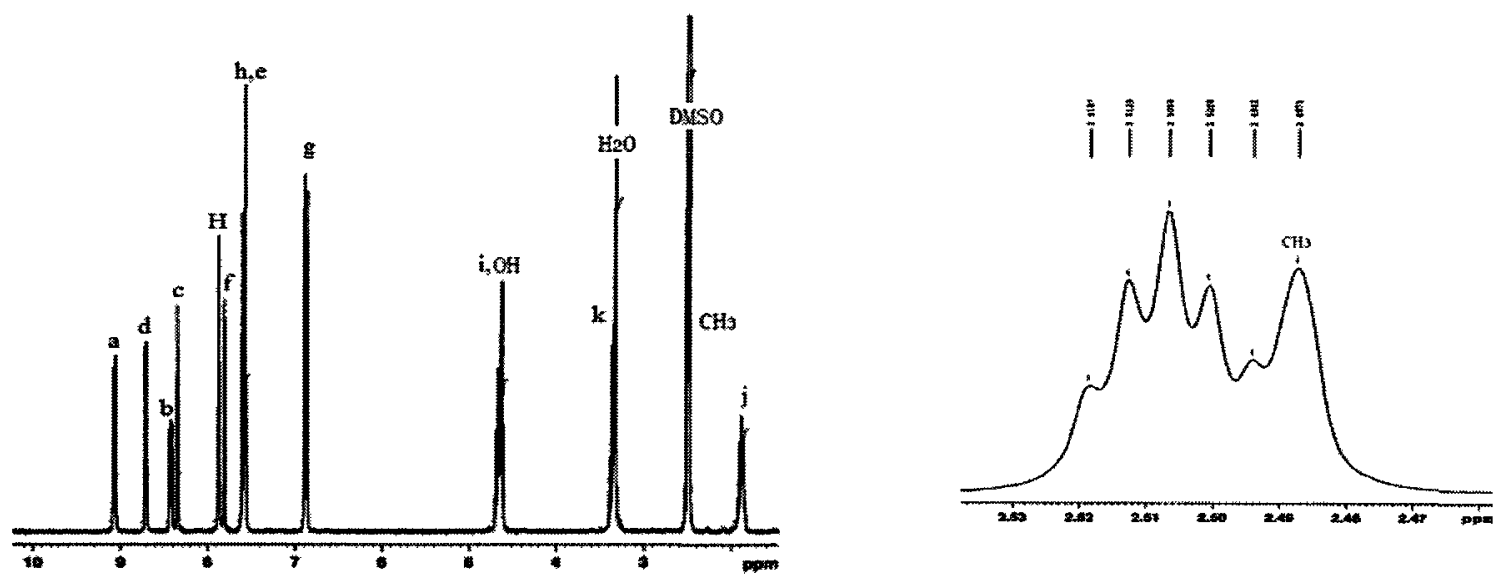

Figure S2.15. ${ }^{1} \mathrm{HNMR}$ of PpQ DM-C3OH in DMSO-d $\mathrm{d}_{6}$.

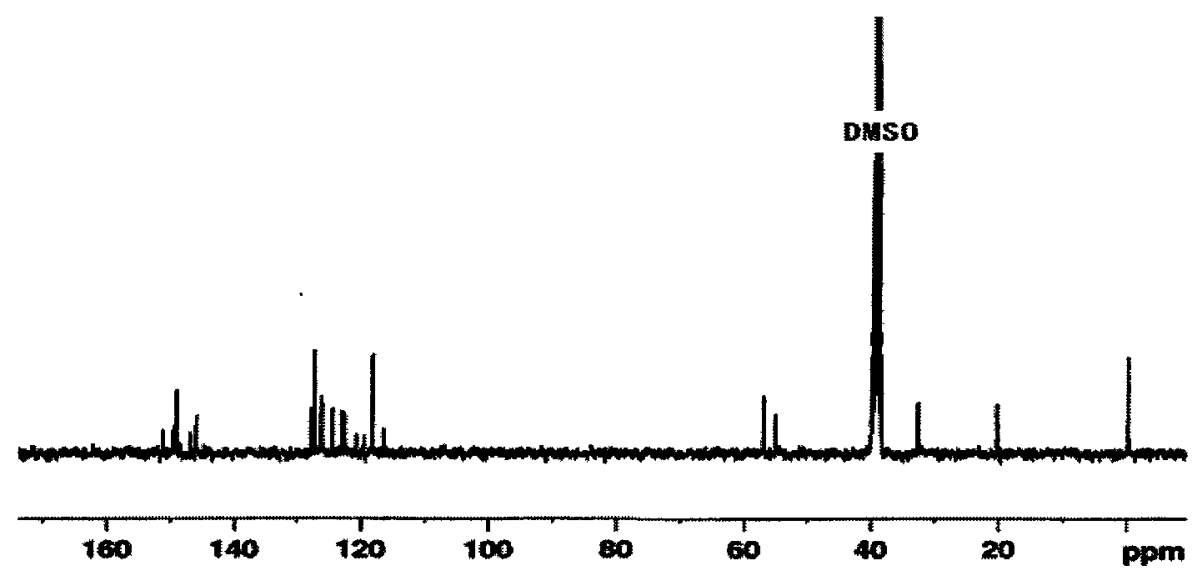

Figure S2.16. ${ }^{13} \mathrm{CNMR}$ of PpQDM-C3OH. 


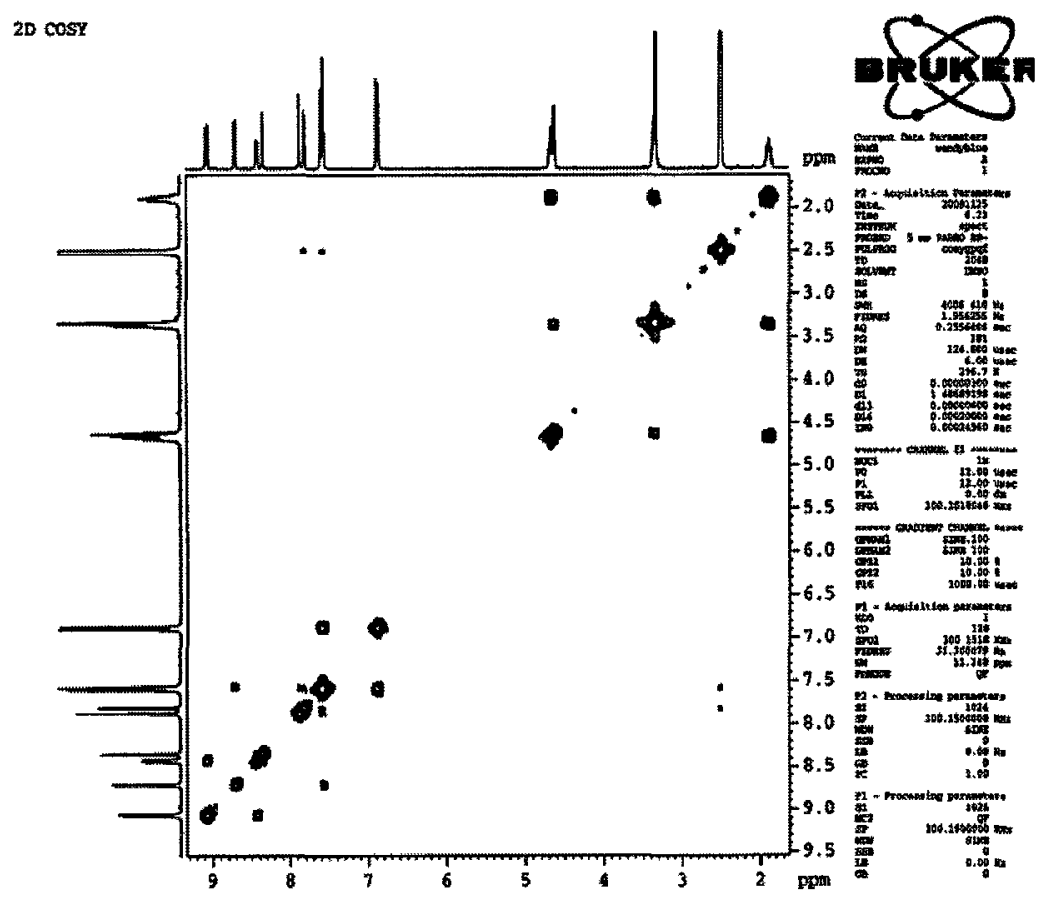

Figure S2.17. 2D cosy NMR of PpQDM-C3OH.

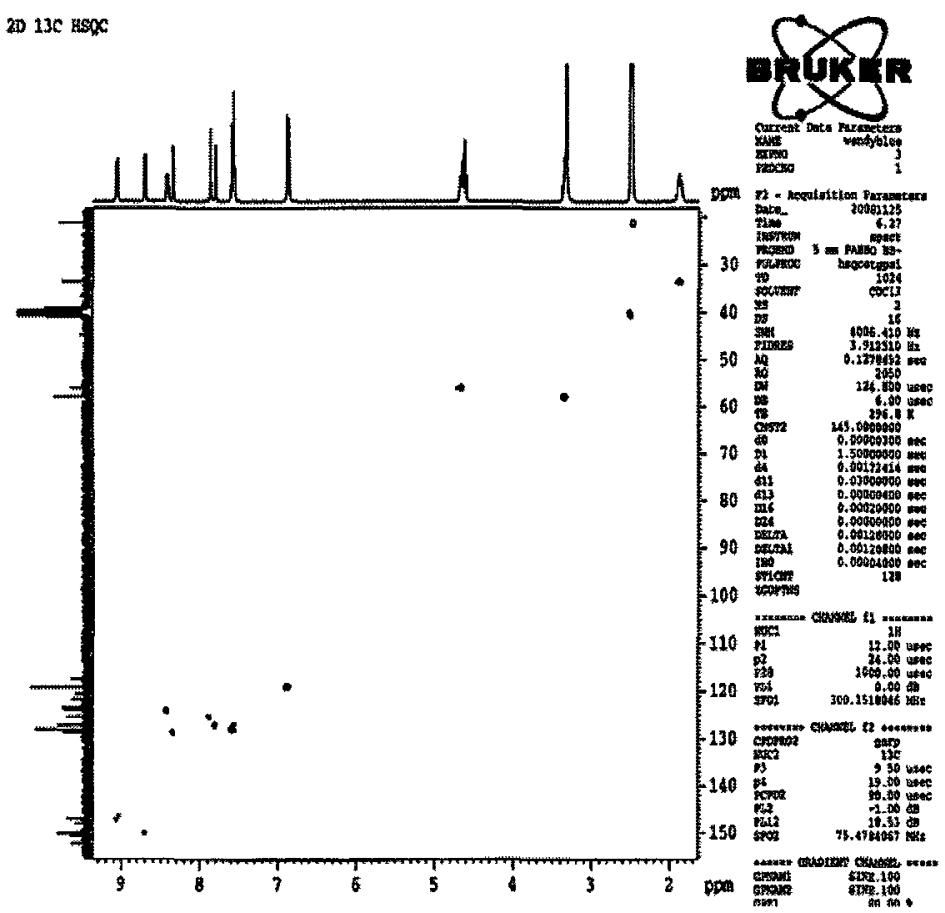

Figure S2.18. 2D 13C HSQC NMR of PpQDM-C3OH. 


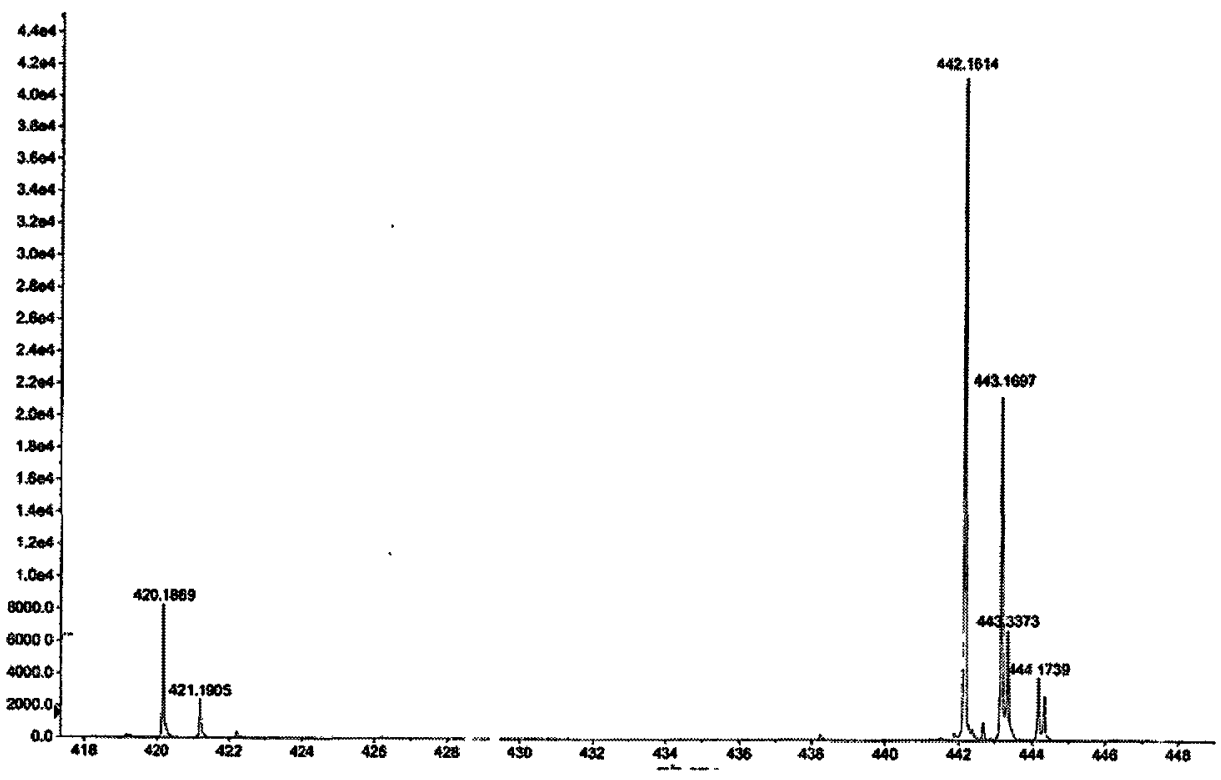

Figure S2.19. ESI mass spectrum of PpQDM-C3OH.

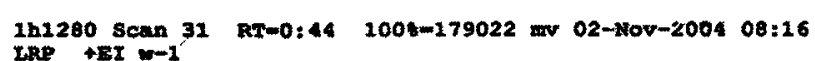
LeP $+\mathrm{Br} w-1$

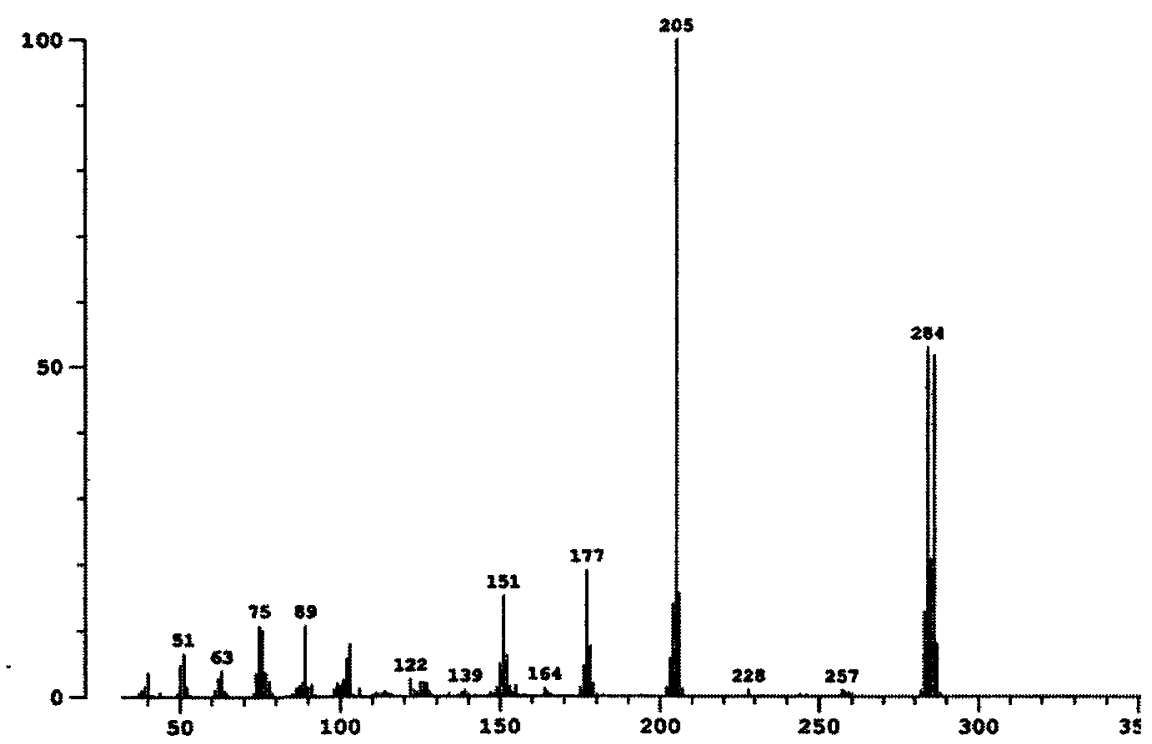

Figure S2.20. EI mass spectrum of compound 1. 


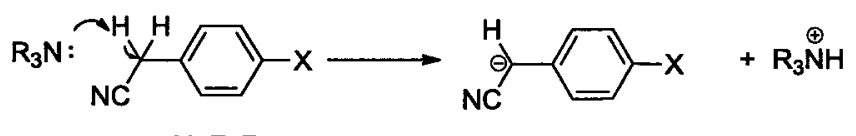

$\mathrm{X}=\mathrm{F}, \mathrm{Br}$<smiles>[R10]NCOC(c1ccncc1)C(C)(C)c1ccc([X])cc1</smiles>
$\mathbf{R}_{3} \stackrel{\oplus}{\mathrm{NH}}$
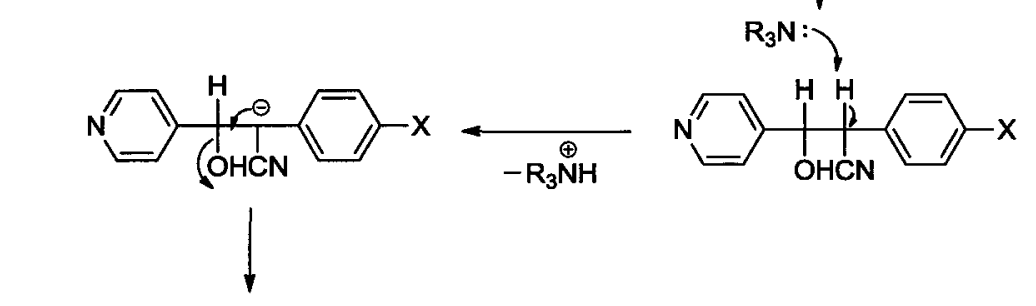

(c)
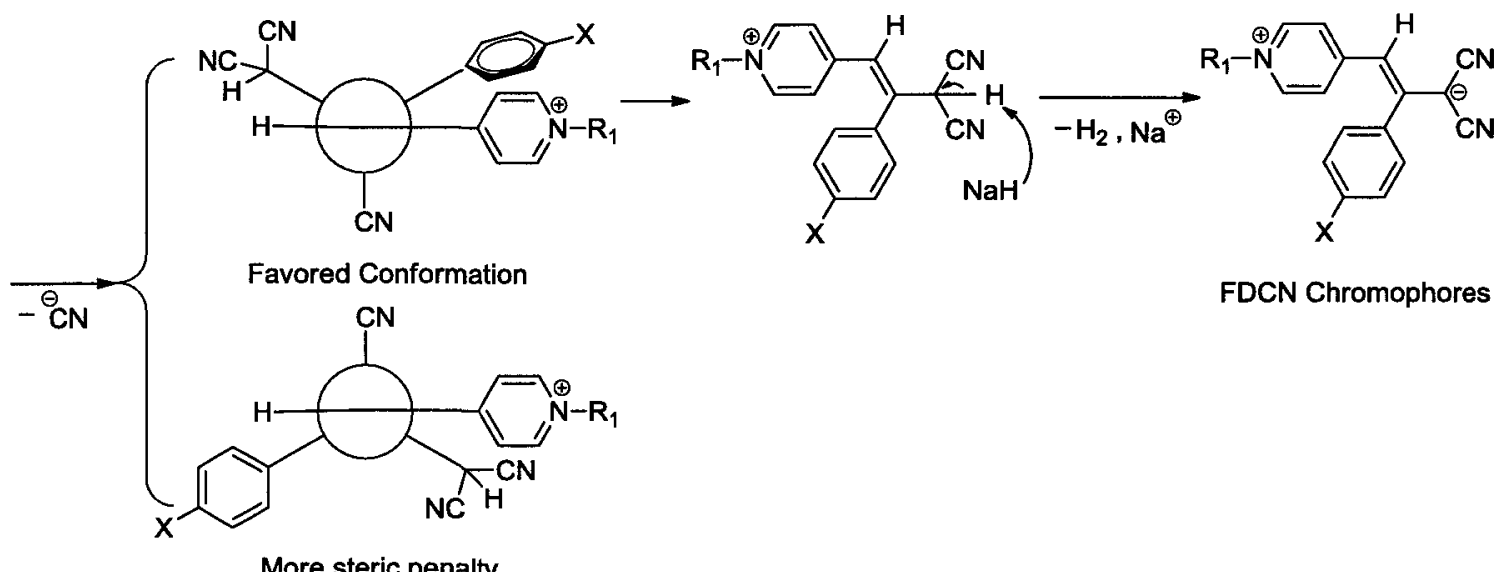

FDCN Chromophores

More steric penalty

Scheme S2.1. Proposed reaction mechanism for the formation of compound 1 and FDCN chromophores 


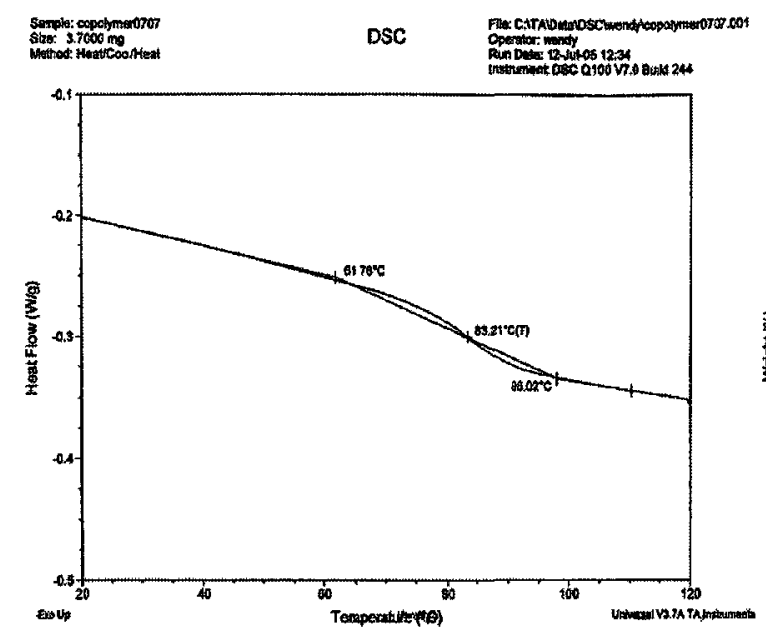

Figure S3.1. DSC trace of A1-1.

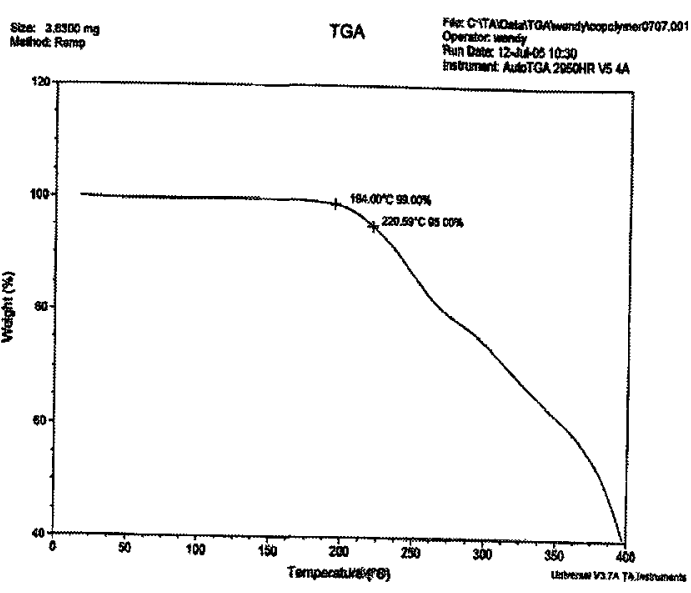

Figure S3.2. TGA trace of A1-1.

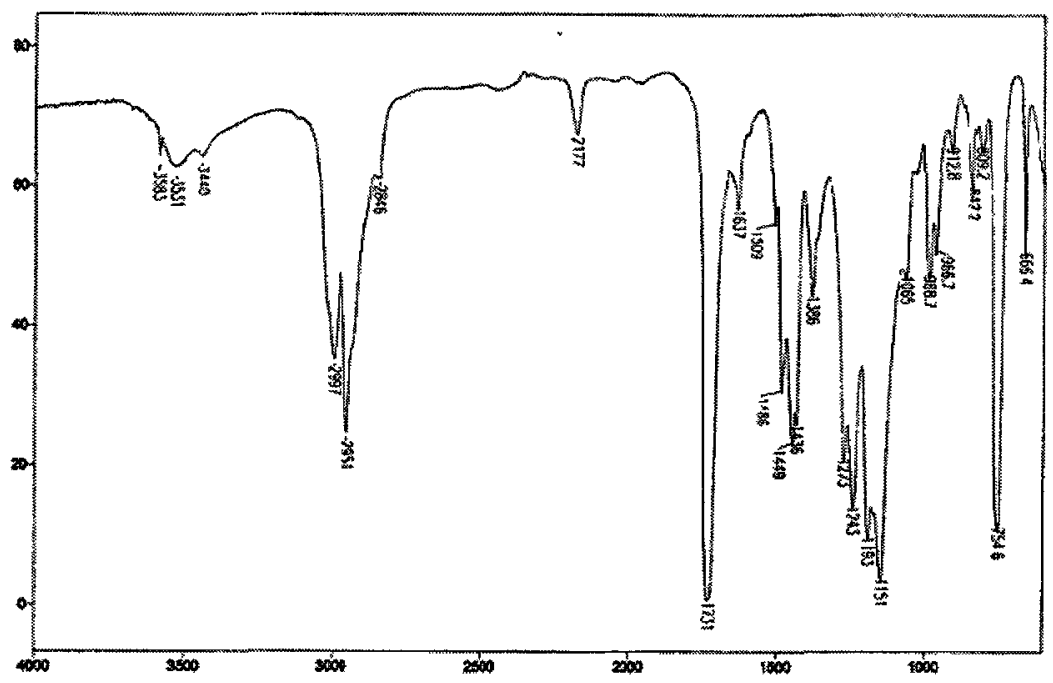

Figure S3.3. IR spectrum of NLO polymer NA2b. 


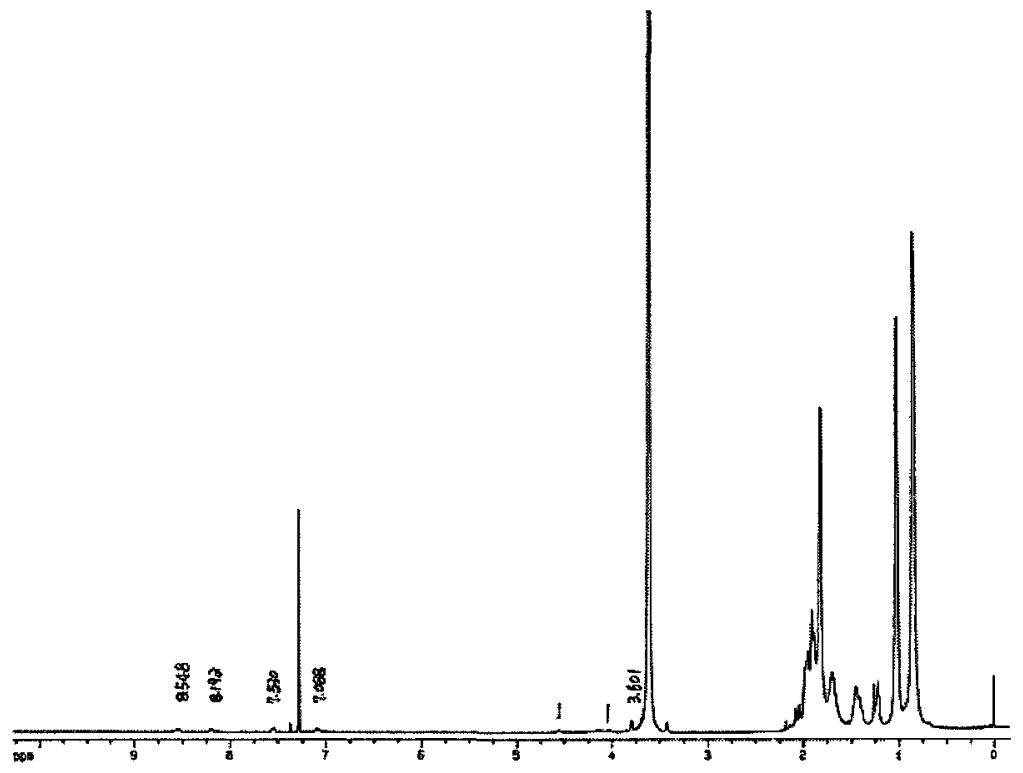

Figure S3.4. NMR spectrum of NLO polymer NA2b.

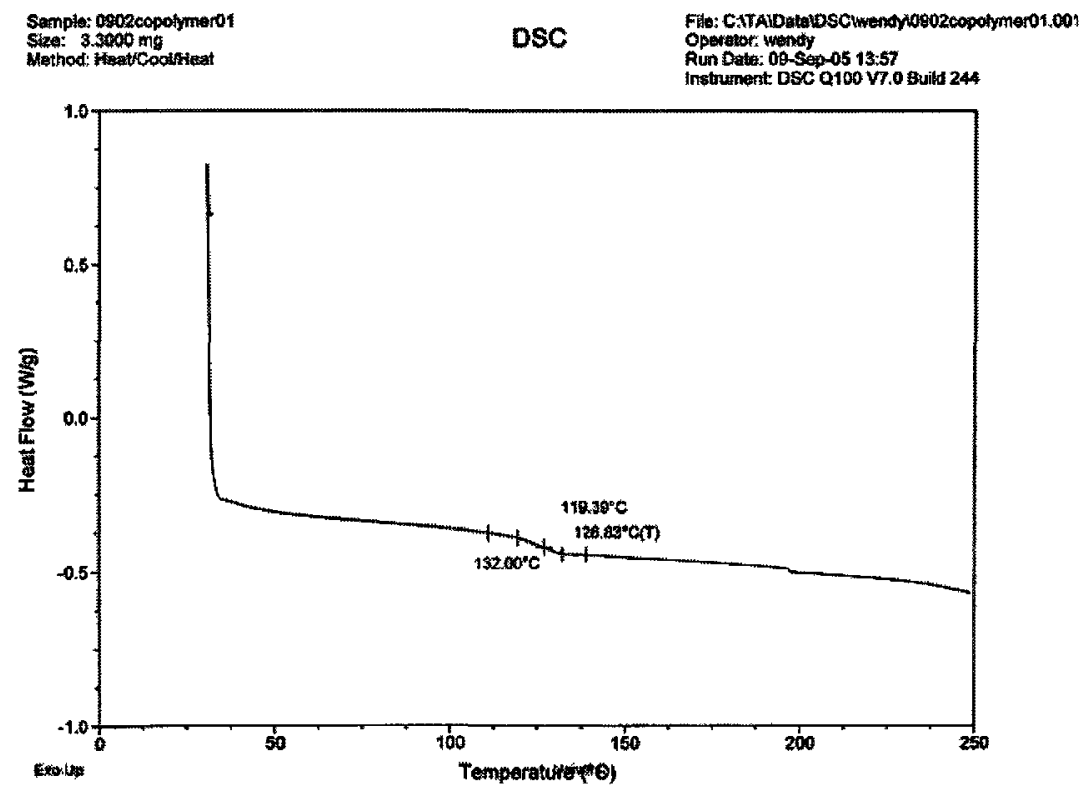

Figure S3.5. DSC trace of NLO polymer NA2b. 

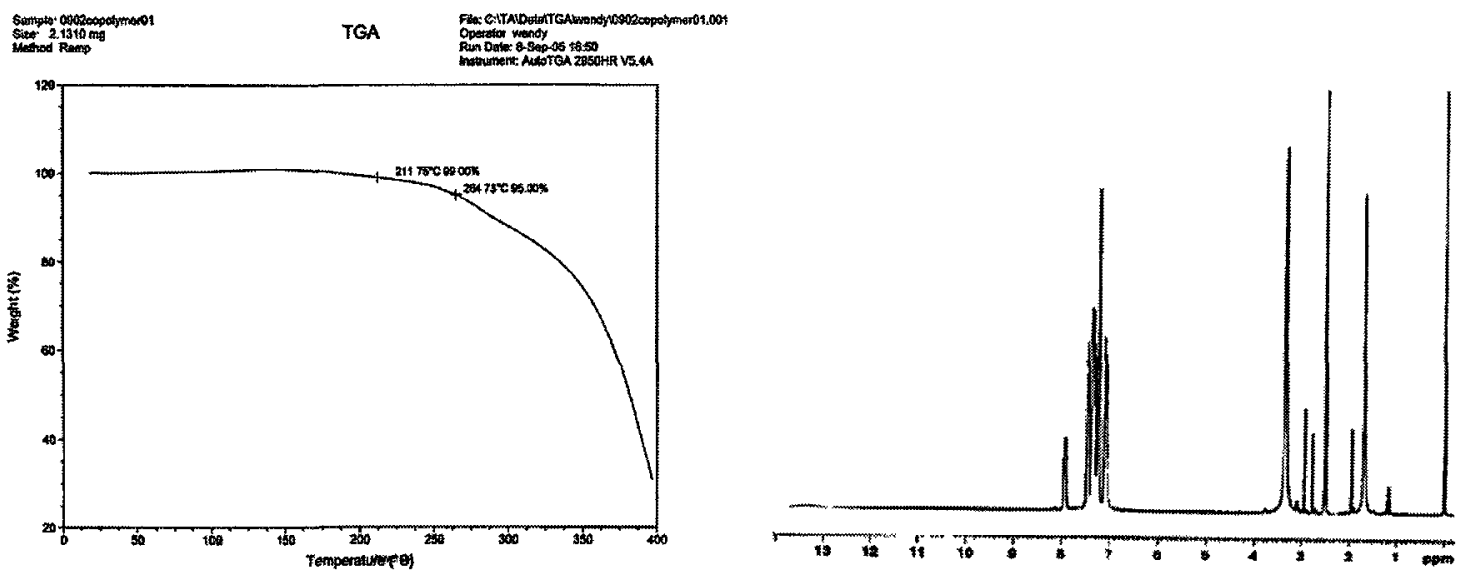

Figure S3.6. TGA trace of polymer NA2b. Figure S3.7. NMR spectrum of PI-1b.

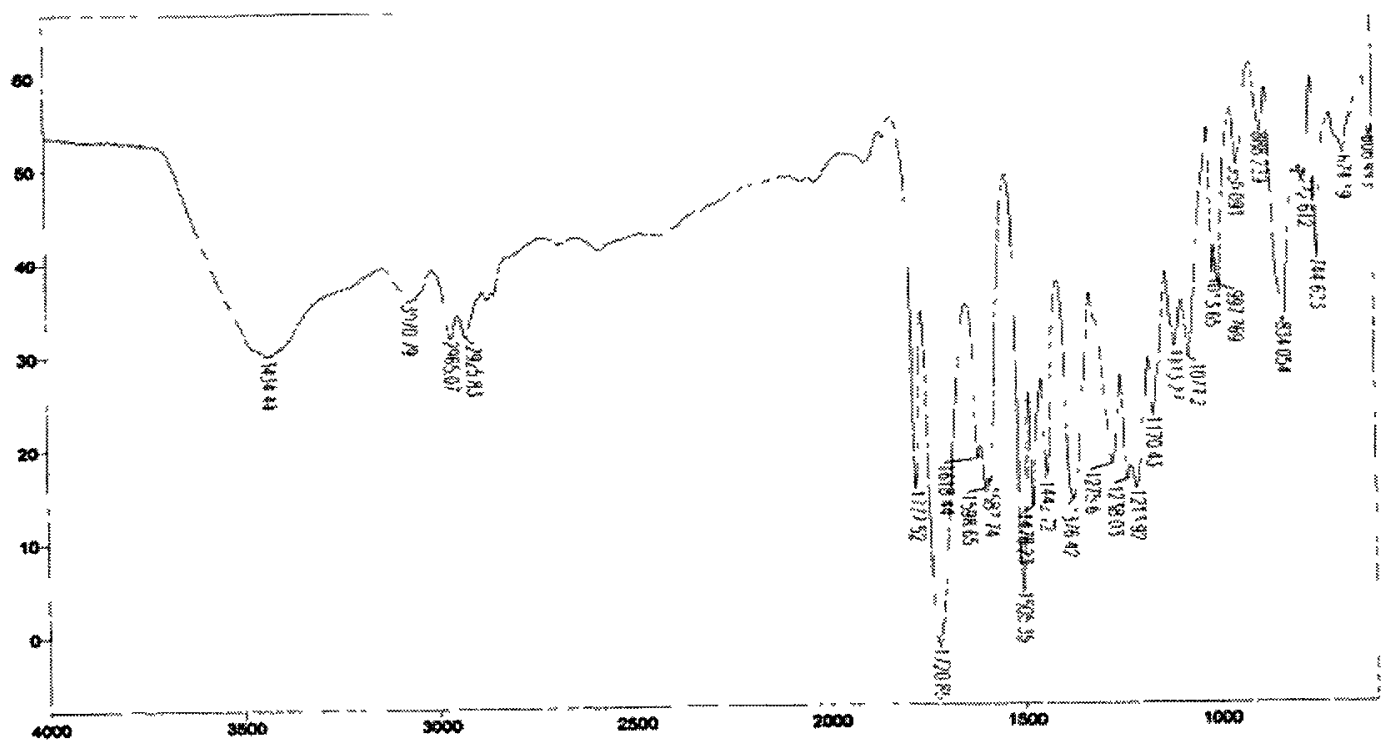

Figure S3.8. IR spectrum of polyimide PI-1b. 


\section{Appendix C Supporting Information of Chapter 4}

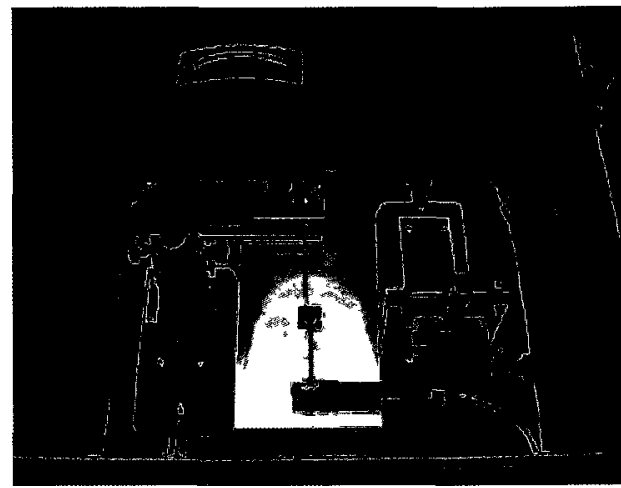

Figure S4.1. Electro-thermal poling station.

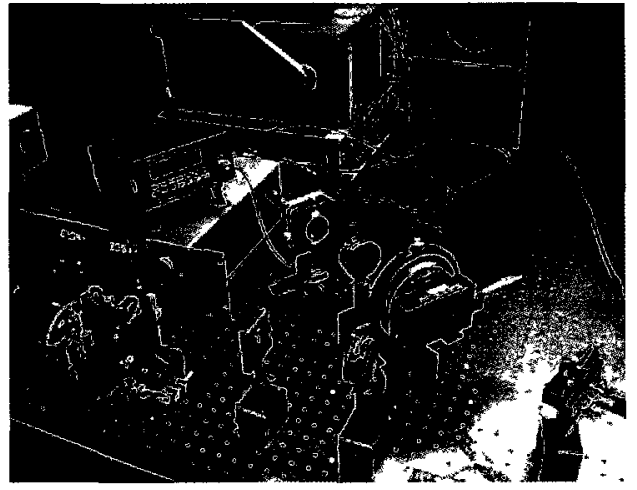

Figure S4.3. Teng-Man Setup.

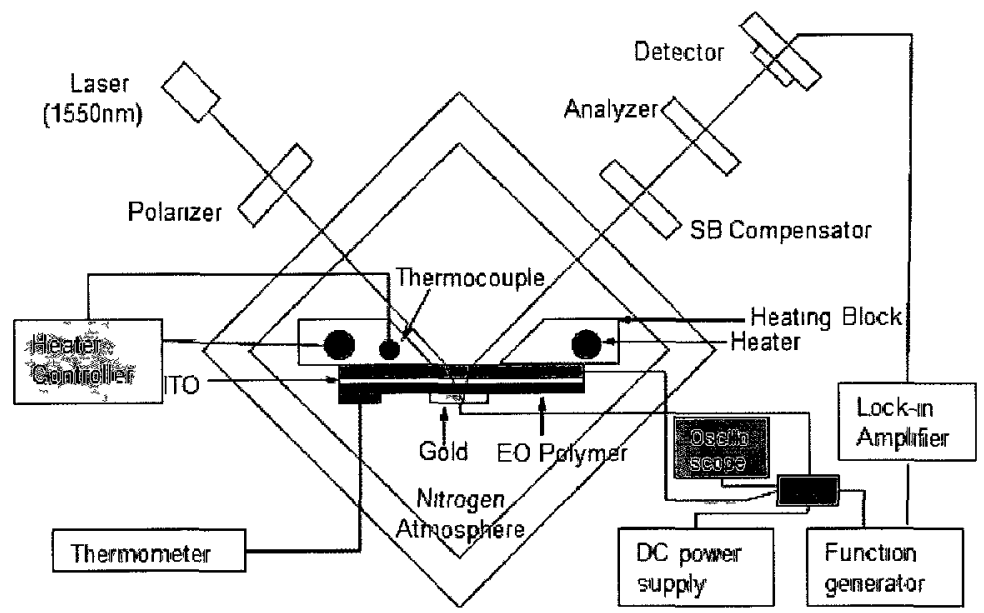

Figure S4.4. The modified reflection ellipsometry apparatus for in situ monitoring of $r_{33}$ during electric field poling.
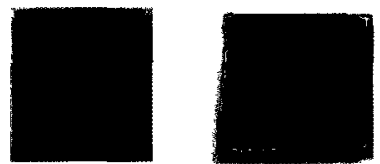

Figure S4.2. Films of FDCN/PES (left) and NPI-d (right) on ITO with gold.
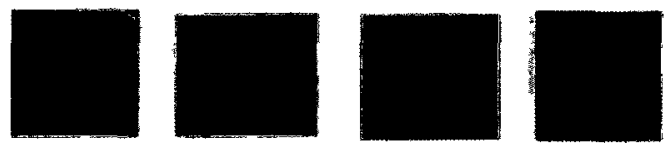

Figure S4.5. Films of PpQDM-Met in different polymers. 


\section{Appendix D Supporting Information of Chapter 5}

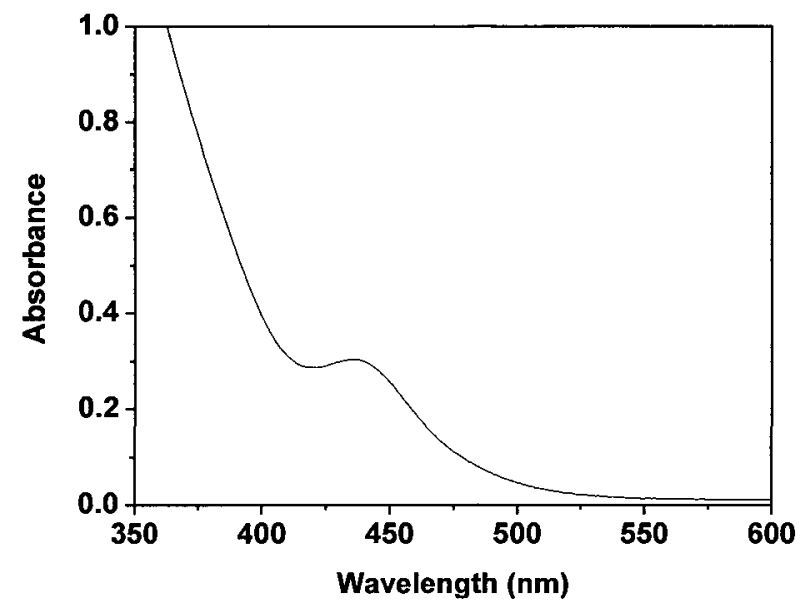

Figure S5.1. UV-Vis spectrum of M1-Cu.

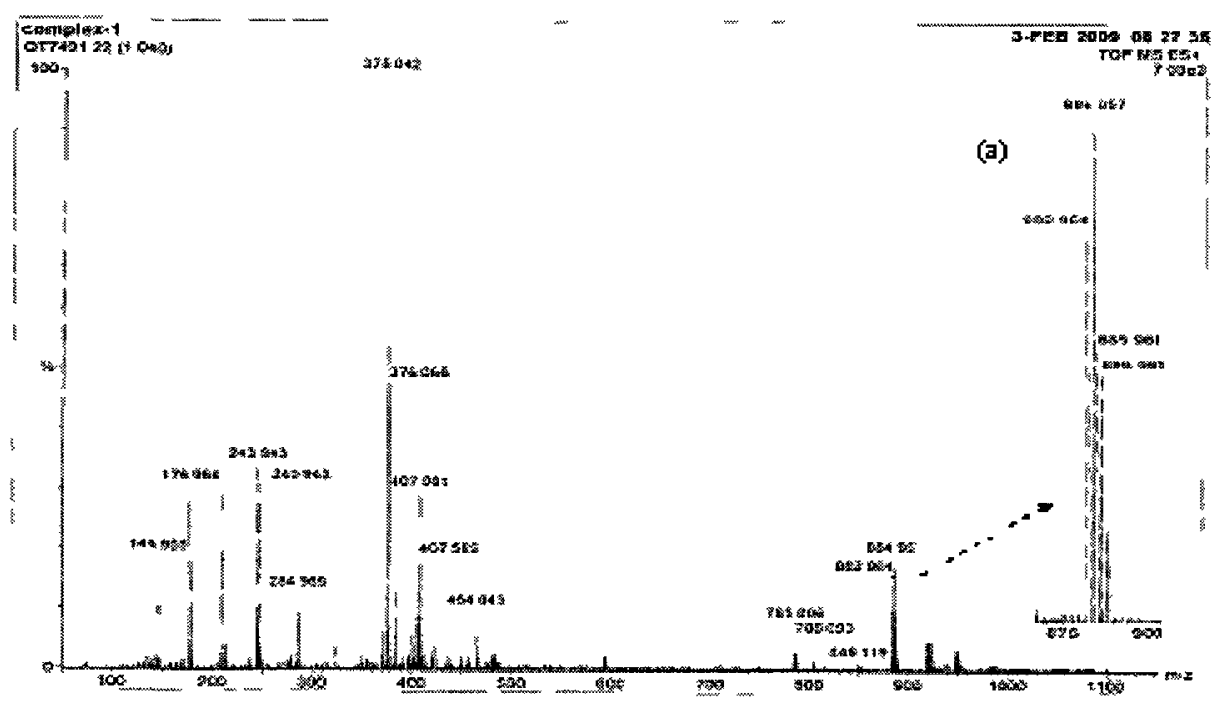

Figure S5.2. ESI mass spectrum of M1-Cu. Inset: (a) expanded peak of the complex. 

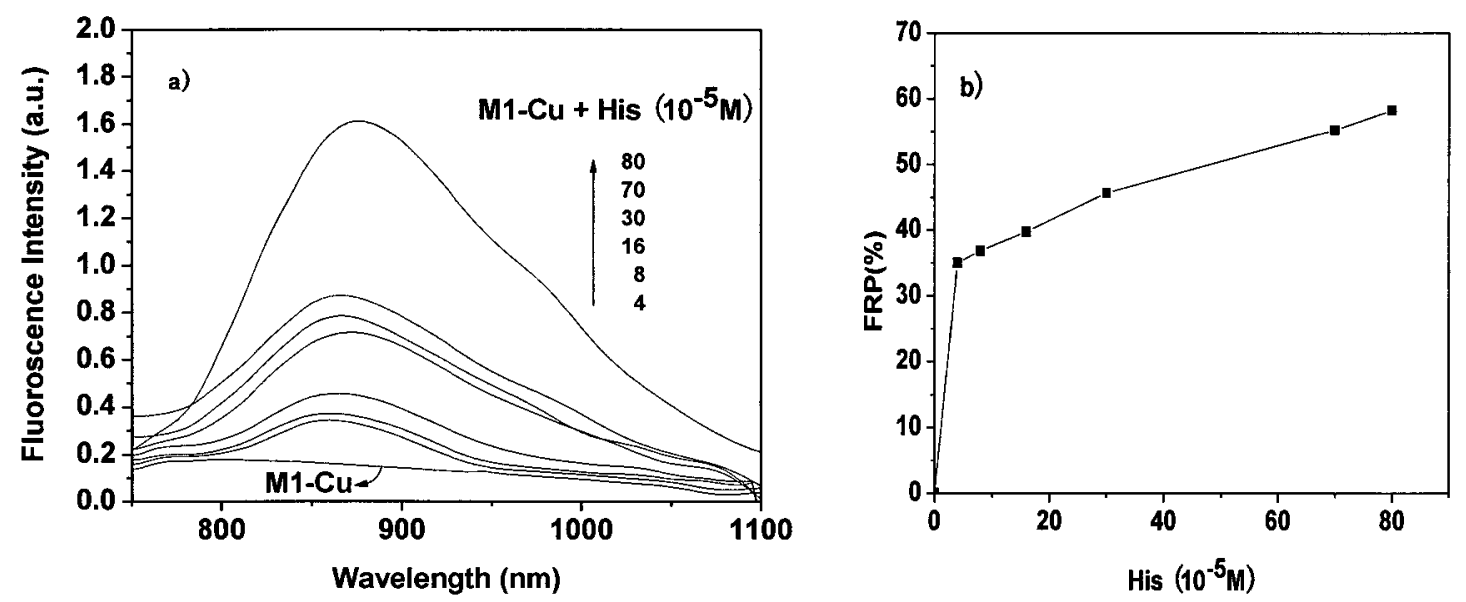

Figure S5.3.1 a) Changes in the fluorescence emission spectra of M1, M1-Cu and fluorescence titration of M1-Cu with increasing amounts of His. The concentration of M1 is fixed at $2 \times 10^{-5} \mathrm{M}$. Excitation wavelength $(\mathrm{nm}): 670$. b) FRP versus the concentration of His. Each spectrum is acquired $1 \mathrm{~min}$ after His addition.
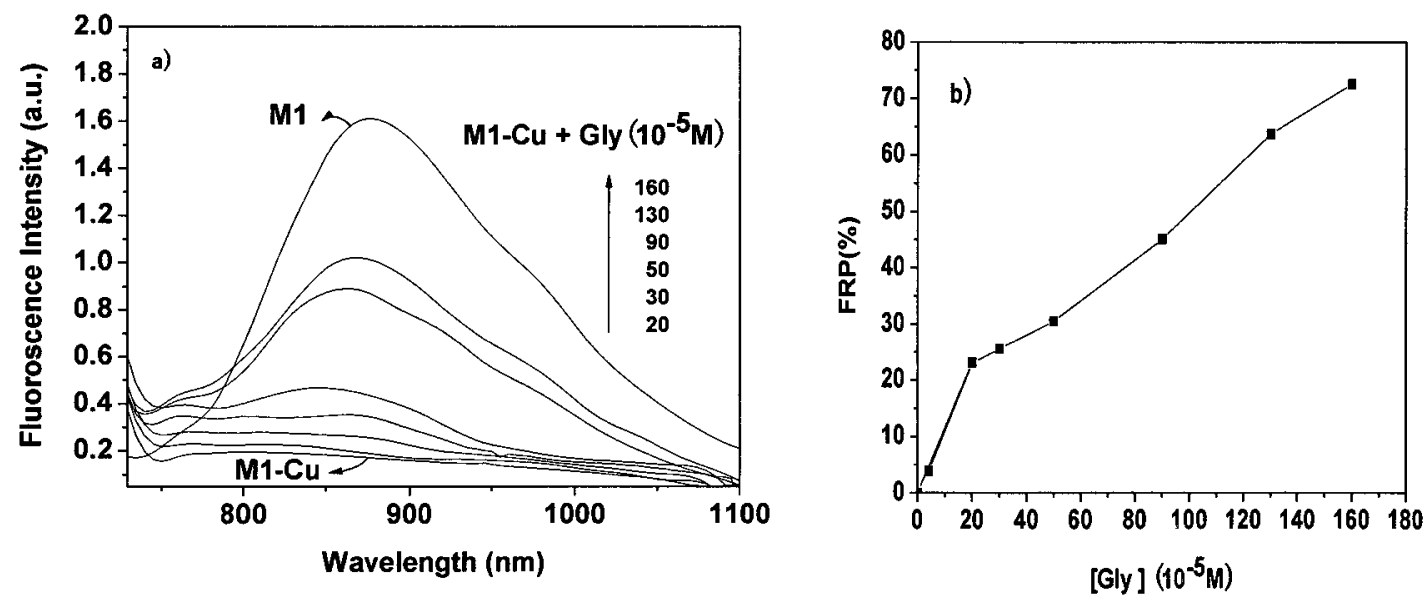

Figure S5.3.2. a) Changes in the fluorescence emission spectra of M1, M1-Cu and fluorescence titration of M1-Cu with increasing amounts of Gly. The concentration of M1 is fixed at $2 \times 10^{-5} \mathrm{M}$. Excitation wavelength (nm): 670. b) FRP versus the concentration of Gly. Each spectrum is acquired 1 min after Gly addition. 


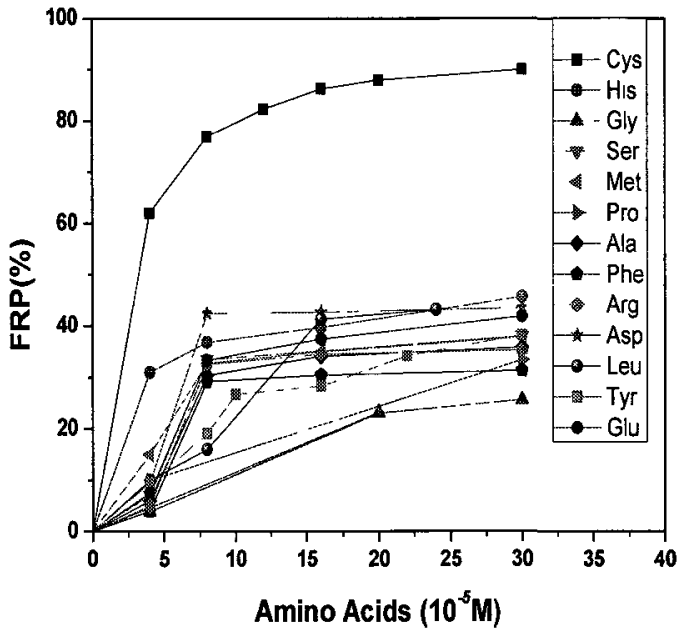

Figure S5.3.3. FRP with respect to the concentration of different $\alpha$-amino acids.

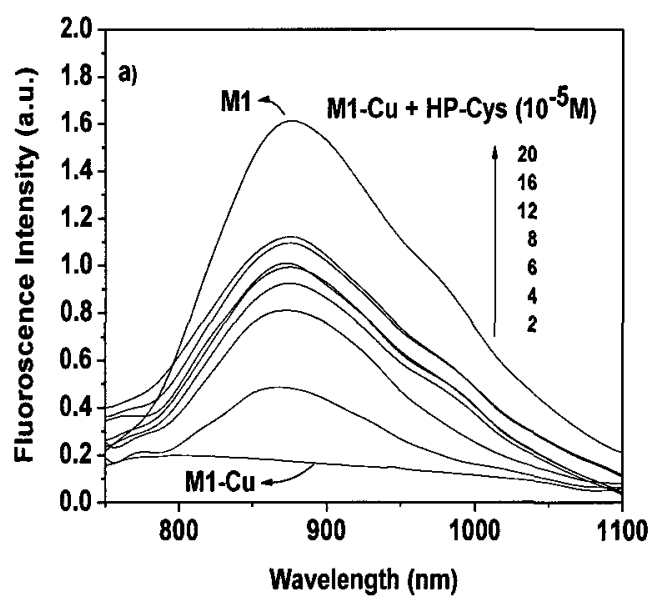

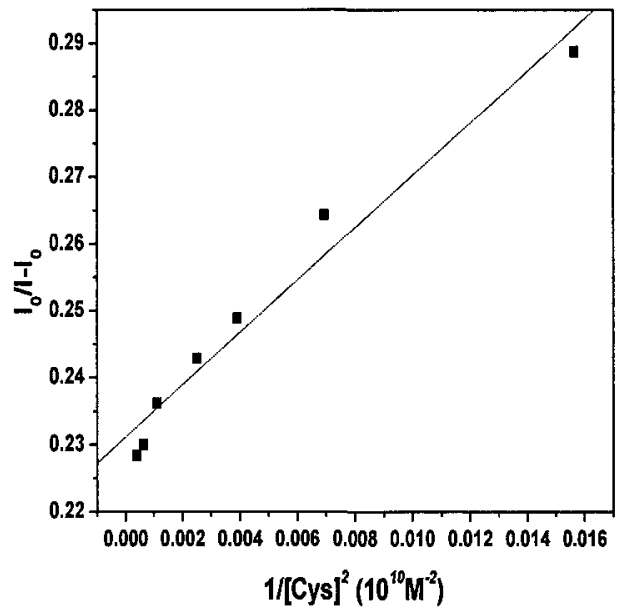

Figure S5.4. B-H plot of M1-Cu with Cys.

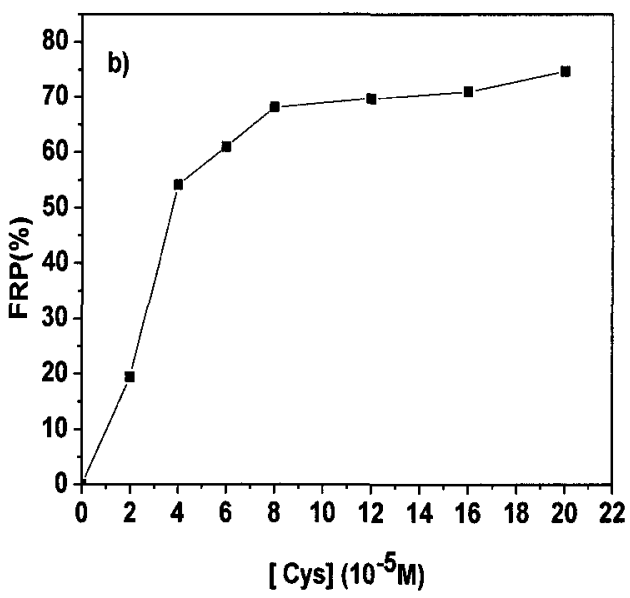

Figure S5.5. a) Changes in the fluorescence emission spectra of M1, M1-Cu and fluorescence titration of $\mathbf{M 1 - C u}$ with increasing amounts of human plasma with Cys. The concentration of M1 is fixed at $2 \times 10^{-5} \mathrm{M}$. Excitation is at $670 \mathrm{~nm}$. b) FRP versus the concentration of human plasma with Cys. 


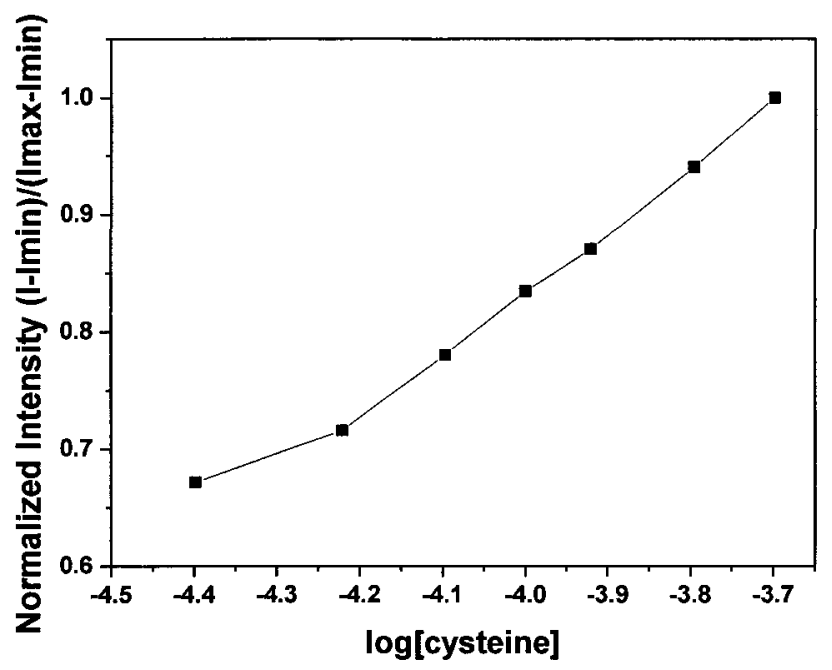

Figure S5.6. Fluorescence intensity of the M1-Cu probe at each concentration of Cys in human plasma, normalized between the minimum fluorescence intensity, found at zero Cys and the maximum fluorescence intensity, found at $20 \times 10^{-5} \mathrm{M}$ Cys in human plasma. The excitation at $670 \mathrm{~nm}$ and the emission at $875 \mathrm{~nm}$.

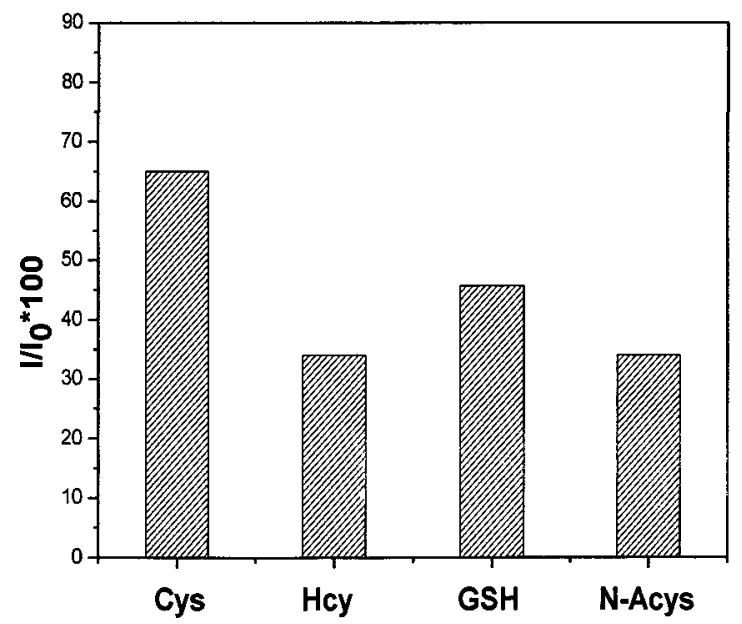

Figure S5.7. Fluorescence emission response of M1-Cu towards various bioactive thiols. Concentration of $\mathbf{M} 1$ is $2 \times 10^{-5} \mathrm{M}$; concentration of bioactive thiols is $4 \times 10^{-5} \mathrm{M}$. 